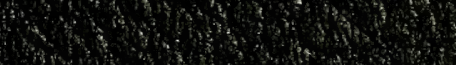

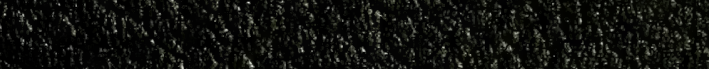

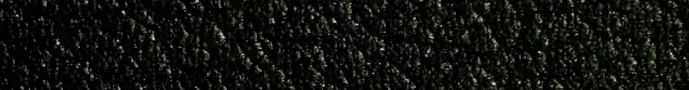
(1)

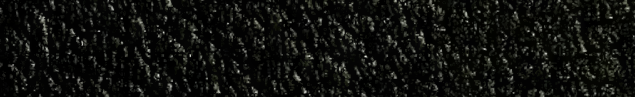

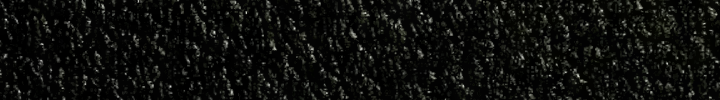

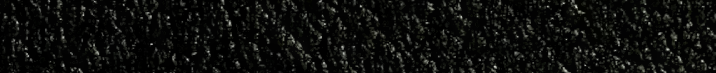

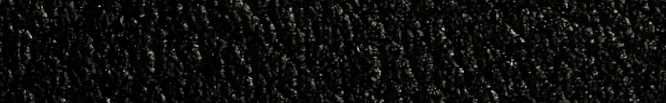

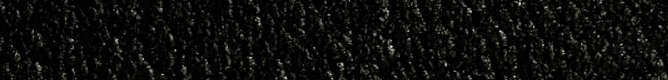

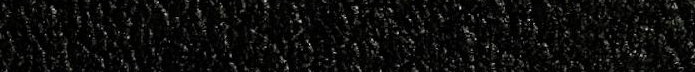

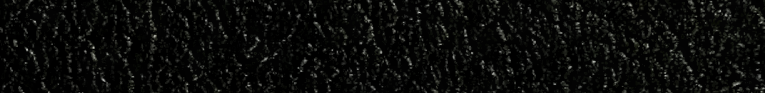
W 


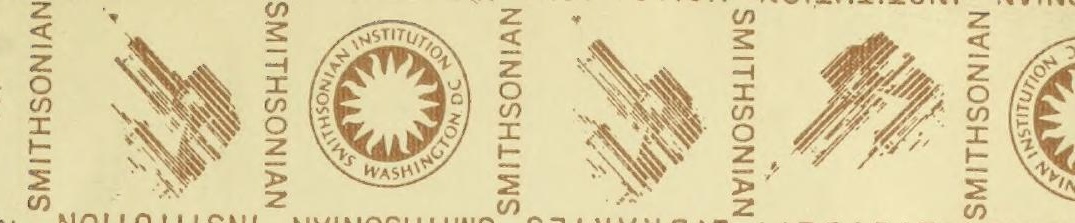
N

(N)
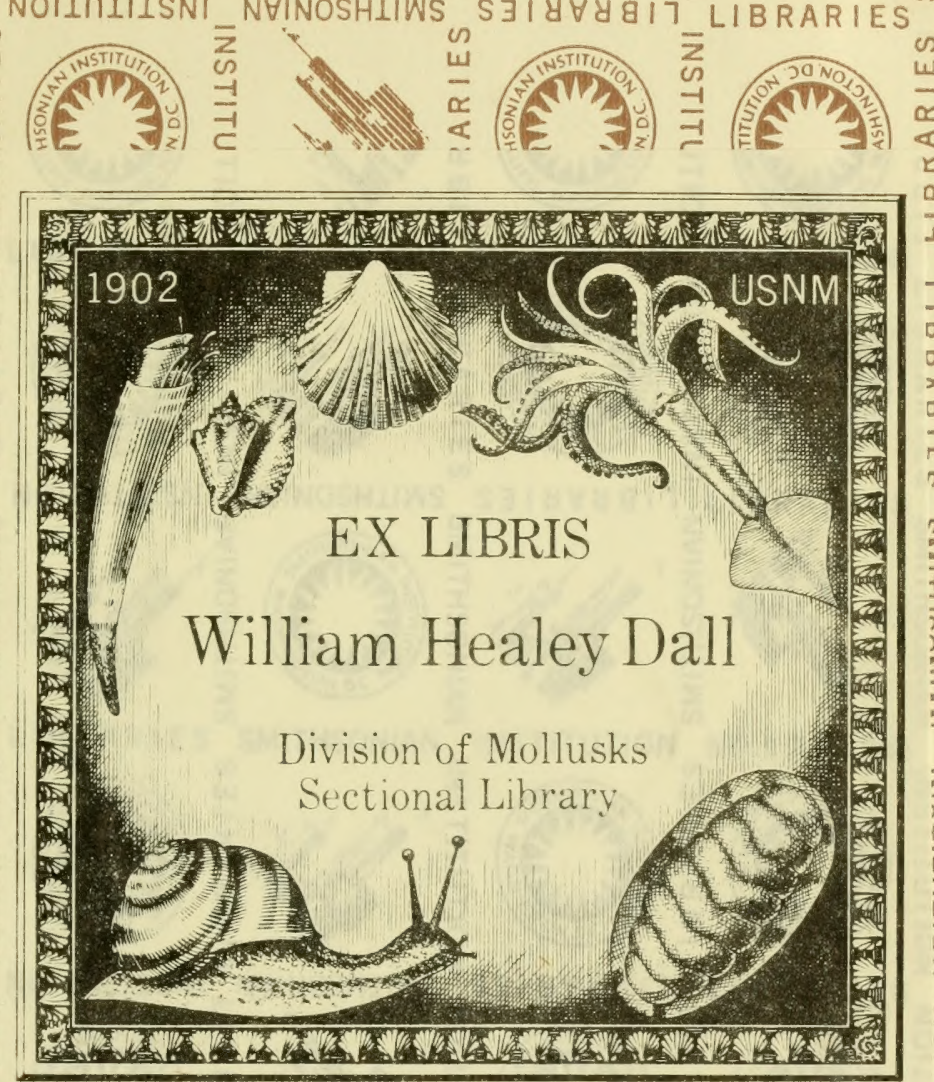

NHINC

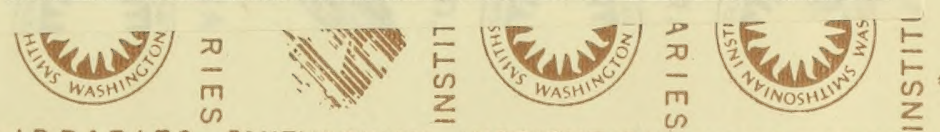

SMITH

LIBRARIES SMITHSONIAN INSTITUTION NOIDIIISNI

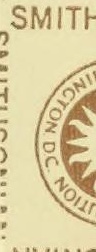

NHINC

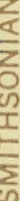
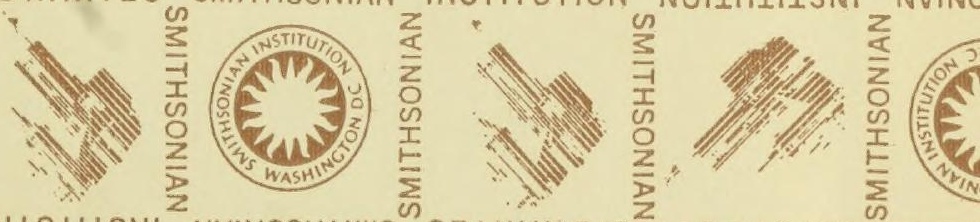

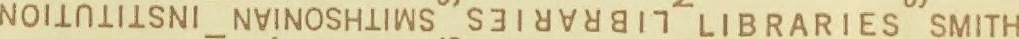

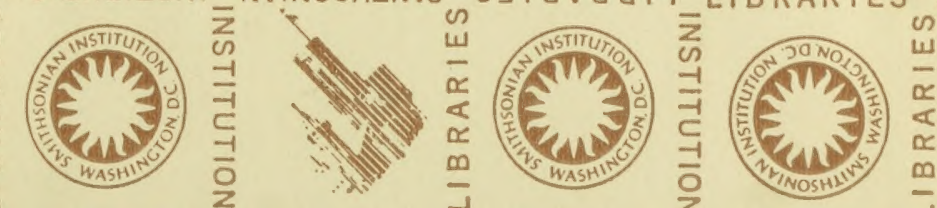






\section{Datiotion of Mollundos}

CONCHYLIOLOGIE SYSTÉMATIQUE.

GOQUILLESUNIVALVES, CLOISONNÉES. 


\section{SOUS PRESSE,}

LES COQULles Dissivalves, MULTivalves ET HIVALVES

DE L'IMPRIMERIX DE L. HAUSSMANN, RUK DE LA HAIPE, No. 80. 


\section{CONCHYLIOLOGIE SYSTÉMATIQUE, \\ E T \\ CLASSIFICATION MÉTHODIQUE DES COQUILLES;}

OFFRANT LEURS FIGURES, LEUR ARRANGEMENT GÉNÉRIQUE, LEURS DESGRIPTIONS CARACTÉRISTIQUES, LEURS NOMS ;

AINSI QUE LEUR SYNONYMIE EN PLUSIEURS LANGUES.

Ouvrage destiné à faciliter l'étude des Coquilles, ainsi que leur disposition dans les cabinets d'histoire naturelle.

Impenderimus horas.

COQUILLES UNIVALVES, CLOISONNÉES.

\section{PAR DENYS DE MONTFORT,}

Ancien Naturaliste en chef de S. M. le Roi de Hollande, pour les Indes Orientales, etc., etc.

TOME PREMIER.

$$
\text { PARIS, }
$$

Снвz F. SCH OELL, LIBAIRE,

RUE DESFOSSÉS-SAINT-GERMAIN-L'AUXERROIS, No. 29. 1808 . 
Nnl exemplaire ne sera reconnu s'il n'est revêtu de la signature ci-bas : les conditions vonlues par les lois ont été remplies.

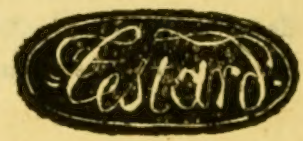




\section{DISCOURS \\ PRÉLI MINAIRE.}

S I létude en général est pleine de charmes pour l'homme qui a cultivé les sciences en s'élevant jusqu’à un certain degré de connoissances, nous devons cependant avouer que rien n'est aussi plus hérissé de difficultés dans ses commencemens. Presque partout les livres élémentaires se font encore désirer, parce qu'il est extrêmement difficile de tracer, de développer des élémens d'une manière aussi précise que claire et succincte, et que, d'un autre côté, un semblable ouvrage ne peut être le fruit que de veilles longues et laborieuses, et qu'il ne peut être produit que par un écrivain assez profondénent consommé dans les matières qu'il traite, pour les metire à la portée de tous ceux à qui il destine un livre, entrepris dans le but de leur applanir la route qu'ils désirent de parcourir ; d'y guider leurs premières démarches, de façon à ce qu'ils n'aient jamais à y faire un pas mal assuré ou rétrograde; de la nettoyer pour eux d'une foule d'obstacles; et propre enfin à leur en épargner toutes les aspérités. Les livres qui traitent des 
sciences ne nous manrpuent point, à la rérité; mais ces sciences ont pris un tel essort de nos jours, chacune d'elles se divise en tant de rameaux, et se rattache a tant de branches, qu'i l'égard de quelques-mes d'elles, la vie de l'homme n'est plus assez longue pour les creuser it fond ; et, la multiplicilé des ouvrages publiés successivement, qui viennent s'entasser les uns sur les autres, et dont il est absolunent nécessaire de comnoitre au moins la majeure partic lorsyuion veut parler en connoissance de cause, cette muliplicitr elle-meme semble aujourd'hui ajouter de nouvelles difliculés à celles yui existoient dïja. Car, si d'un cûté elle projetle, clle propage les lumieres, de l'autre elle suppose les mojens at Ia facilité de se procurer ces ouvrages, et elle exige des facnltís ordinarement au-dessus de celles des hommes pour qui les scinnces sont un objet d'études sérieuses et suivies, ou de télassement daus des fonctions pénibles. È parmi ces sciences, il en est même quelques-unes où une fortunc, fùt-clle assez considérable, ne suffroit point pour se procurer une foule d'ourrages, souvent tris voluminenx, tries-dispendieux, imprime's avec le plus grand luxe, et qui traitent des natieres que l'on veut appoliondir. Ce n'est meme que dans les cités populeuses el capitales que lion peut jonir de ees bibliotheques pul,iques, dues à la munificence des souverains, ct a la protection puissante qu'ils accordent aux arts et it ceux qui les cultivent. Ce n'est que dans ces bibliothè ques scules fue lihomme rui cherche à sinstruire peut espérer de rencontrer prespue tous les secours quil désireroit vainement ailleurs. C'est encore aussi dans ces villes principites queles communications entre les sarans sont 
plus franches et plus amicales que dans les villes secondaires; où plus isolés, ils ont aussi en général plus de réserve; réserve et isolement qu'on ne retrouve que trop souvent, et bien malheureusement, surtout hors de France, dans des contrées toutes entières. Voilà en partie les motifs qui nous ont porté à entreprendre l'ouvrage actuel, dans l'espoir de le rendre élémentaire et classique, propre à tenir lieu d'une quantité d'autres. Il est systématique, et c'est après l'avoir long-temps médité, après avoir réuni une foule de matériaux pour sa confection, que nous nous sommes enfin décidé à sa publication.

Les deux volumes de conchyliologie que nous mettons au jour, et qui renferment les genres des coquilles univalves cloisonnées et non-cloisonnées, seront immédiatement suivis d'un troisième volume qui sera consacré aux coquilles multivalves, dissivalves et bivalves; la réunion des trois formera une conchyliologie complète, en offrant l'ensemble systématique des genres connus jusqu'à ce jour. Cet ouvrage fait lui-même partie d'un vasteplan, qu'à la vérité nous ne pouvons point nous flatter de terminer seul; car il embrasse toutes les productions de la nature, et successivement nous publierons de même des parties détachées, mais complètes chacune en soi, de ses trois règnes; de manière à ce que chacune d'elles soit entière, en formant cependant, quoiqu'isolée, une partie de ce vaste ensemble. Nous aimons à croire que l'exemple que nous donnons sera suivi, et que limpulsion une fois imprimée, des plus mes plus exercées que la nôtre, pourront parcourir 
cette carriere d'une maniere bien plus brillante que celle que nous employons. Cependant, dès ce dúbut nos soins et notre zile, notre amour pour la science, pourront nous concilier lindulgence de nos lecteurs, et nous espérons quils voudront bien sourire à ce produit de nos travaux; travaux, nous le répétons, qui n’ont ćé entrepris quapres descétudes aussi suivies que profondes, ainsi que de longues et de mûres réflexions.

Toutes les figures de cet ouvrage ont été dessinées et gravées par nous; d'autres auroient pû y mettre plus d'art, mais moins de vérité; c'est pour avoir acquis l'intime conviction qu'en général les livres d'histoire naturelle laissent beaucoup, et souvent même presque tout à désirer eu égard au dessin, que nous avons voulu étudier cet art cn naturaliste, dans l'espoir de racheter par l'exactiturle les effets dopposition, les aspects pittoresques, le fini et les accidens d'ombre et de lumiere que nous abaudomnons aux peintres de profession. La tiche du naturaliste moins poétique et bien plus gènće, est de faire ressortir les moindres détails, soin minutieux qui nuit à l'cflet de l'ensemble; et parmi ces détails, il doit pescr. principalement sur ceux caractéristiques, en les faisant ressortir au lieu de les laisser noyés dans le vague ou dans la masse. Cependant il ne doit pas entierement nigliger toutes les parties accessoires, ainsi que ces reflets de lumieres qui donnent aux objets récrées par ses jinceaux, la rondeur, le mouvement et la vic. D'un autre colti, l'expérience nous a convaincu qu'on ne sanroit, pour impriner les objets dars la mémoire, parler it trop de sens réunis, et quilil est presque moralement 
impossible de ne point se ressouvenir de ce qu'on a vu, de ce qu'on a lu, et de ce qu'on a saisi et compris toutà-la-fois; des dessins multipliés sont donc d'une nécessité absolue, parce qu'ils en disent bien plus encore que les phrases les plus claires, et qu'ils parlent aux yeux. On ne peut point s'en passer dans un livre consacré à l'étude de l'histoire naturelle, et dans ce cas son auteur seul sait rendre vivement et avec le sentiment de la vérité, ce qu'il a vu, ce qu'il a senti. Il est donc indispensable qu'un naturaliste sache dessiner : il faut qu'il puisse par une bonne figure donner le complément de ses écrits, de ses vues, de sa pensée; et nous osons dire que n'eût-il que de médiocres talens en ce genre, lui seul est en état de le faire. D'ailleurs son dessin n'est-il point indépendant des fautes et des contresens que nous voyons faire tous les jours par des dessinateurs excellens, nais étrangers à cette étude ? et de quelle manière leurs dessins déjà infidèles, ne sont-ils pas encore plus mal rendus par un graveur étranger de même à la chose, et obligé d'exécuter les mêmes idées au moyen d'autres instrumens, et sous d'autres modifications ? Dès-lors cette gravure doit ressembler en quelque sorte à ces discours, qui passant de bouche en bouche, reçoivent autant d'altérations qu'il existe d'individus qui les propagent; car chacun y mettant du sien, ils finissent par perdre toute leur signification et leur intégrité. Le naturaliste doit donc s'efforcer, non-seulement à dessiner, mais encore à graver lui-même tous les objets qu'il soumet aux yeux de ses lecteurs. Mais aussi sous ce rapport quel temps et quelles dépenses n'exigcroient point de lui ces devoirs, s'il les exécutoit de la manière 
qui est aujourd'hui génćralement recue pour tous les ouvrages dans lesquels on accompague le texte par des planches d'un grand luxe et en taille-douce?

Cest done pour surmonter toutes ces difficultés que nous nous sommes dicidé à mettre en regard de notre texte des gravures en bois. Quoi(que difficiles dans leur 'xécution, elles n'exigent pas autant de soins, ni autant de dépenses que des gravures en cuivre; elles s'impriment instantanement avec le texte, et par des moyens ' qui leur sont communs avec lui; elles durent aussi plus long-trmps, parce qu'elles ont moins à souffrir que des planches de mital; et quoiqu'elles laissent beaucoup à désirer du cúté du fini et de l'élégance, nous osons esperer qu'clles satisforont par celui de leur vérité et de leur exacritude. Quant à l'économie, elles nous permettront, quoiqu'aussi nombreuses que le sujet l'exige, d'offrir enfin au public des livres vraiment élémentaires, arec tout le bon marché possible, et qui pourront encore tenir lieu d'une immensité d'ourrages sur les mímes matieres, ctremplacer par une synonymic exacte de volumineuses bibliothéques.

Dans ces rues, et comme il devient assez indifférent par ruelle classification systématique des étres on commence celle générale des productions de la nature, et que d'ailleurs on pourra successivement les placer aur rang que l'un croira leur ètre respectif, rang qui rependant n'est pour nons que de convention; au licu d'entamer notre plan par les animaux les plus apparens, ceux qu'on me convemu d'appeder les plus parfaits, tels que le sont 
l'homme et les quadrupèdes; nous avons préféré de commencer par des parties moins connues, et qui laissoient plus, ou mème tout, ì désirer. Actuellement nous publions les coquilles, enveloppes calcaires des mollusques testacés; immédiatement après ces premiers volumes, nous pourrons mettre sous presse la classification systématique des vers, des polypes, des radiaires et des crustacés, de façon cependant que l'on puisse séparer chaque partie de l'ensemble, la rejeter dans la masse, ou en faire l'acquisition isolément et d'après le genre d'étude qu'on aura adopté.

Les mollusques testacés forment une classe très-nombreuse des animaux sans vertèbres; il est vrai que dans une marche catégorique nous aurions pu les faire précéder par les sèches, parles poulpes, et par les calmars ou mollusques simplement coriacés; mais ce; animaux, et d'autres mollusques constamment nus, formeront l'objet d'un travail séparé. On peut regarder celui que nous publions sur les mollusques testacés, comme faisant. l'initiative dans l'immense série que nous avons entrepris de parcourir. La classification systématique et générique des coquilles qui appartiennent aux mollusques testacés, sera donc le commencement, et fera partie de la classification générale des animaux sans vertèbres, sans charpente osseuse; seconde division des corps vivans, mais cependant et incontestablement la plus nombreuse des deux qui coupent tout l'ordre animal. Nous croyons que M. de Lamarck est le premier qui, saisissant de larges données, ait tranché tout l'ordre animal en animaux à vurièbres et en animaux sans vertèbres, et cette 
division a été depuis généralement arloptéc par les naturalistes, sans que ccux-ci aient cependaut indiqué à qui on en étoit redevable: il nous étoit réservé de rendre ceite justice ì un savant illustre, aussi éclairé que laborieux, dont nous avons suivi les lerons, et ì qui, sous me multitude derapports, la srience a tant d'obligations.

Dans lourrage actud nous n'avons envisagi que d'une maniere tr's-secondaire les animaux constructeurs des coquilles, et méme nous n'avons figuré aucun d'entreux ; ces volumes sont particulierement destinés a leur classification par linspection de leur tèt extéricur, ou des piéces testacies quils recèlent, soit sous leurs manteaux, soit dans lintérieur de leurs corps; ct conme cliarque coquille figurée forme a elle seule, avec le texte qui l'accompagne, un article isolé, tout naturaliste peut, en les transposant à son gré, opérer parmi elles toutes les mutations, et se former par leur secours un ordre systématique particulice, et un systéme qui lui soit personnel, si celui que nous lui présentons ne se concilic point son suffagc. Dailleurs, ces animaux sont encore trop peu commus pour pouvoir les saisir dans leurs dítails, dans leurs nunurs et dans leur ensemble; et, jusgua ce que nous ayous plus de dommées a cet egard, nous croyons pour le moment quiun systeme uniquement basé sur l'organisation de ces animaux, seroit extrèmement incomplet. Leurs revêtemens, leurs teits ou leurs corquilles sont done encore les seuls types constans, invariables et conums qui puissent nous servir ¿ lus classer jusqu'a présent ; parce quiils sont constam- 
ment et uniformément les mêmes, et que la facilité de leur conservation a puissamment coopéré à la formation des collections si multipliées qu'on en a faites de tout temps.

Le nombre des coquilles maintenant connues peut s'élever, ainsi que nous le croyons, à plus de six mille espèces ou variétés; c’est après en aroir étudié la majeure partie que nous nous sommes décidé, en marchant sur les traces des conchyliologues célèbres, ct principalement sur celles des Linné, des Bruguière, des Lamarck, de les classer dans leurs genres respectifs; c'est-à-dire, sous des formes, et d'après des types assez tranchans pour décider des points d'arrêt et de repos; familles plus ou moins nombreuses, dans lesquelles les espèces qui en constituent les membres viennent d'ellesmêmes se ranger. Dans ce travail, nous avons presque toujours dû considérer l'ensemble deschoses, saisir une foule d'aperçus, chercher à débrouiller une certaine confusion, et nous rendre clairs, de facon, à ce que jamais une coquille ne vînt à nous arrêter au milieu de notre marche dans une route dẻjà jalonnée, mais que nous avons voulu entièrement applanir. S'il étoit un moyen d'arriver à ce but, on ne pouvoit y parvenir qu'en adoptant une marche simple, et qui fût d'autant plus laborieuse à tracer. C'est en présentant successivement chaque espèce à tous les genres, que nous avons reconnu si elle y étoit reçue, ou si elle en étoit repoussée ; si elle s'y plaçoit naturellement, comme d'elle-même, ou si elle en étoit rebutée. Lorsqu'elle ne s'assimiloit à aucun de ceux que nous avons reconnu ou établi gra- 
duellement dans cel onvage, dés-lors en sisolant, cette coquille est venue sans effort former la tite d'un nouveau genre à établir, et simulamiment d'autres quilui itoient congéneres, sont venues de méme se ranger à côté d'elle.

Nous croyons done en ce moment pouvoir nous flatter que d'aprés notre systime, la classification et l'arrangement d'une collection de coquilles, eil la supposant aussi considerable que possible, devient aussi facile que simple, et qu'il n’est aucune de ces coquilles maintenant comnues qui ne se place, comme d'elle-mème, dans les genres dont ces rolumes oftient actuellement la nomenclature. On pourra nous reprocher peut-être de les avoir un peu trop multiplićs, mais la chose éloit nécessaire pour ne pas accoler et ranger, comme on ne l'a que trop fait jusqu'à présent, sous les mêmes caractères, des coquilles disparates, qui mème forcément ne veulent point se prêter à cette violence; et nous sera-t-il permis d'alléguer de noureau en notre faveur qu'un systême quelconque n'est qu'un moyen factice et de convention, imaginé pour soulager l'esprit et la mémoire, et afin de se rendre compte d'objets nombreux par une classification tantót plus et tantót moins étendue? Tous nos efforts ont donc été employés afin de pouvoir envisager la nôtre comme basée sur des caractères assez tranchés pour espérer d'avoir atteint enfin le but que tous les autcurs qui traicerent systematiquement de la conchyliologie siétoient proposés. C'est en resserrant trop, en cumulant les objets, quion devient obscur, qu'on reste dans le vagne et daus l'indé. 
cision, et ne royons-nous pas que successivement le besoin de s'entendre a fait créer de nouveaux genres aux dépens des anciens déjà établis, comme nous le prouvent les ouvrages de Linné, de Bruguière, et comme le confirment les travaux des auteurs qui de nos jours marchent encoresur les traces de ces illustres devanciers? D'ailleurs si même quelquefois nos lecteurs trouvoient que nous eussions excédé à cet égard de certaines bornes, illeur est très-facile dans ce cas, de regarder d'après leur opinion, la figure d'une coquille que nous donnons pour type de genre, comme ne présentant que celui d'une espèce, et sous ce rapport, notre travail seroit encore utile, parce qu'il présenteroit toujours une figure exacte, et pour ainsi dire typique, qui plus tard serviroit à la publication et à la reconnoissance de ces mêmes espèces.

Sous ces points de vue, notre ouvrage reste donc élémentaire, parce que nous avons voulu le faire de manière à ce qu'il présentât un genre d'utilité réelle, et qui lui fût propre. Il peut d'un côté, en aplanissant la voie, faire naître le goût de l'histoire naturelle, même chez l'enfance, en lui offrant à côté d'un texte court, concis, et uniquement chargé de faits, des figures qui sont pour elle un langage favori, parce que sans ambiguité, sans circonlocutions, il prés cnte à ses yeux une image des objets; il peut offrir de même un sujet utile d'études pour le jeune homme avide d'acquérir des connoissances, dans un âge qui décide du reste de la vie; mais les hommes instruits sont ceux qui nous tiendront le plus de compte de nos travaux, travaux 
que nous n'avons entrepris que daus la vue de donner de l'extension à la science. Depuis long-temps tous les désirs se portent vers un point de départ fixe et invariable; vers un ouvrage général, et fait pour être compris dans toutes les langues, renfermant des types qu'on fuit invoquer, citer au besoin, sans ambiguité, et présentés de manière à se faire entendre de tous. Ce sont ces hommes instruits qui jugeront si nous sommes entré dans la vraie route, et si, sous le rapport de la conchyliologie, nous avous rempli ce désir général.

Il est une vérité qui, malgré de fréquentes attaques, est restée incontestable, c'est que la culture des sciences honore tous ceux que leur amour posside; et cette culture fera maintenant et constamment partie de l'éducation publique. Parmi ces sciences, celle de l'histoire naturelle brille au premier rang: depuis long-temps des hommes respectables placés à la tête des colléges publics, et chargés par état de cette éducation, avoient senti le besoin de varier les études, et d'ajouter à celle des langues vivantes et mortes, des notions élémentaires sur les êtres divers qui peuplent les airs, le glolie et ses mers. Tels furent entrautres en France les membres de la congrégation de l'Oratoire, successeurs des Jésuites. Ils surent inspirer à leurs élèves non-seulement le goût des belles-lettres, mais encore éminemment celui de l'étude de l'histoire naturelle. Chez quelques-uns d'entre cux ce goût a jeté de profondes racines : mais parmi les productions si nombreuses de la nature dans ses règnes multiplićs, sil en est de plus brillantes, de plus interessantes, qui semblent attacher davantage que dautres 
branches, et appeler plus impérieusement les regards, la conchyliologie, à coup sûr, occupe parmi elles un des premiers rangs.

Et en effet, qui ne connoît point ces riches collections de coquilles que nous offrent presque tous les cabinets d'histoire naturelle, dont constamment elles semblent faire la base, la majeure partie, et le plus bel ornement? Est-il rien qui puisse égaler l'émail de leurs couleurs, tellement scintillantes et variẻes, que dans leur réunion elles disputent d'éclat avec le parterre le plus fleuri? Leurs robes limpides, nacrées et polies, reflètent toutes les nuances, toutes les teintes de l'iris; leurs formes, leur fraicheur ne le disputent-elles pas contre celles des objets les plus beaux ; etrien égale-t-il leur' transparence et leur diaphanéité ? Ces couleurs si belles, si harmonieuses, est-il quelque chose qui les altère? au contraire, il semble que leur possesseur, au lieu de la satiété , leur retrouve, chaque fois qu'il les revoit, de nouveaux charmes. Partout nous rencontrons des collections de coquilles; et aujourd'hui loin d'être ce qu'elles étoient autrefois, un objet de luxe et d'amusement, elles sont devenues pour la science la clef d'une étude vraiment philosophique, en nous indiquant la part que les mollusques testacés eurent et ont encore à la formation du globe que nous habitons. Dans le cours de cet ouvrage on rencontrera successivement une foule d'observations à cet égard, que nous avons toujours émises lorsque l'occasion de parler des coquilles pétrifiées et fossiles, soit analogues de celles à l'état marin, ou sans analogues, est venue naturellement se présenter. 
Les caux des mers, celles des fleuves, des ruisseaux, des lacs et des marais, sont remplies de mollusques testacés; la surface de la terre en est couverte. Encorc plus que la terre et les eaux douces et fluviatiles, l'océan porte sur ses plages, et renferme dans son sein des myriades de familles de ces mollusques : de concert ayec ceux qui construisent et qui habitent les polypiers, ils y élévent des montagnes calcaires en y abandonnant, à l'époque du terme de leur existence, leurs dépouilles testacées : on rencontre des bancs, des côtes entièrement formés par des huitres, par des moules, par une foule immense d'autres coquillages qui rehaussent perpétuellement le fond des mers. Ces mémes corquilles marines se retrouvent aujourd'hui sur les sommets mêmes des plus hautes montagnes de notre globe, ou en général clles constituent d'immenses lits et des couches énormes de marbres et de pierres calcaires, quoiquion les y rencontre aussi quelquefois à l'état siliceux (de pierreà fusil), agatisćcs ou converties en pétrificalions quartzeuses. - La vue de ces corps maintenant pétrifiés et fossiles, autrefois formés par les habitans des mers au sein des eaux profondes, et qui aujourd'hui gissent entassés, pressés les uns sur les autres à des hauteurs oin il semble que les eaux n'aient jamais pu alteindre, a fait rechercher naturellement si parmi, les coquilles marines que nous connoissons actuellement, on ne pourroit pas retrouyer les coquilles analogues et identiquement les mèmes que celles que l'on troure aujourd'hui pétriliées dans ces bancs solides, asséchés, fruits de la cumulation des siécles, et dont on en comnoit qui occupent et forment la base de plus de cent lieues de pays. 
Cette étude fut long-temps infructueuse, jusqu'au moment ou M. Faujas de St - Fond, réunissant ses observations à celles dẻjà émises sur ce sujet, nous a enfin donné, dans son bel ouvrage sur l'histoire naturelle de la montagne de St.-Pierre de Maestricht, une nomenclature de coquilles fossiles parfaitement analogues, c'est-à-dire semblables, à quelques-unes qui revêtent des animaux qui vivent encore aujourd'hui dans le vaste sein des mers, tandis que les premières sont retrouvées à l'état de pétrification, et à un si grand éloignement.de leurs plages actuelles. Voilà les ossemens du globe, et les médailles qui nous restent en preuve de la série incalculable des siècles que la nature entassa pour sa formation. Sans remonter pour le moment à l'origine de ce globe, que nous espérons indiquer un jour lorsque nous publierons notre théorie de la terre, nous ne pouvons, sur nos montagnes et dans nos plaines, méconnoître le séjour de l'océan; et si nous cherchons dans la nuit des temps les causes pour lesquelles nous trouvons aujourd'huices dépouilles marines sur les continens, tout en reconnoissant que ce phénomène est dû à quelque grand cataclysme, à quelqu'effroyable et immense révolution, ne devons-nous point nous demander pourquoi notre globe est incliné de vingt-deux degrés et demi, tandis que, d'après toutes les lois de la pondération, il devroit rouler perpendiculairement à son axe? Alors nous présumerons en connoissance de cause que les partiẹs solides, et maintenant à nu de ce globe, formées primitivement au sein des eaux, se sont dégagées d'elles, et se sont mises à sec dans un horrible choc qui, en détruisant tout aplomb, est venu à cette 
époque désastreuse, établir un nouvel ordre de choses.

Lorsque le globe, debout, rouloit en pondération sur son axe, un printemps perpétuel le pénétroit constamment de ses vivifiantes influences. A cette époque des iłges, une température chaude et procríatrice régnoit sur toute sa surface, et les itres et les mollusques testacés qui habitent encore aujourd'hui les zônes chaudes étoient répandus sur toute sa superficie. De-là les ossemens d'éléphans et de rhinocéros, animaux des pays chauds, dont la Sibcric et les terres du nord sont pour ainsi dire parées; de-là ces coquilles de la mer du sud, et de celles de l'Asie, que l'on trouve aujourd'hui fossiles en Europe, et qui ne vivent maintenant que dans les latitudes brûlantes; de-là ces coquilles pélagiennes pétrifiées, c'est-à-dire, autrefois habitantes du fond des hautes mers, qu'elles ne quittent point, en raison d'une organisation qui leur est particulière, fait attesté par quelques-uns de ces mollusques encore vivans, arrachés ou enlevés au sein des mers profondes, tantôt par le jet d'une sonde, et tantôt par quelqu'autre accident, qui sont venus orner nos cabinets, et nous donner de nouvelles lumieres. Tout nous prouve encore que successivement nous retrouverons une foule d'analogues, actuellement que la science marche d'un pas ferme et assuré. Chaque voyage entrepris dans le but des découvertes, ou de l'instruction, ne nous procure-t-il point de nouvelles coquilles, des êtres encore inconnus pour nous, que l'on troure dans des mers et sur des plages neures, ou qu'on n'avoit pas suffisamment explorées? 
Et si d'un autre côté, beaucoup de ces coquilles fossiles, quoique ayant une identité presque parfaite avec celles actuellement marines, offrent cependant quelques différences légères qui font encore hésiter sur leur entière ressemblance; qu'on daigne donc remarquer que cette différence existe aujourd'hui dans les individus marins, décidément et incontestablement de la même espèce, qu'on rapporte des différentes parties du globe. Les coquilles de la Nouvelle-Hollande, ne sont-elles pas en général plus épaisses et plus colorées que celles des Antilles et de la mer des Indes, quoique certaines musiques, des cérites, des vis, des strombes y soient intégralement de la même espèce ? La discussion ne règne donc ici que du plus au moins; et d'ailleurs pouvons-nous assurer que dans la série des temps ces formes n'essuieroient jamais quelques modifications, soit légères, ou plus marquées? Ces formes seroient-elles constamment et invariablement les mêmes au milieu de vicissitudes perpétuelles de vie, de non-existence, de propagation et de repos, de calme et de destruction? Et quelles preuves pourrions-nous donc alléguer en faveur de cette invariabilité, nous qui ne faisons qu'une apparition momentanée sur la terre; tandis que des siècles même d'observations exactes, s'il en existoit, ne seroient rien en comparaison des temps entassés par la nature ? Cependant nous sommes maintenant assez avancés pour quel'étude des coquilles aujourd'hui marines, et de leurs analogues actuellement pétrifiés ou fossiles, nous prouve sans réplique que la terre est sortie des eaux, qu'elles furent antérieures à l'existence du globe terraqué, et que c'est à juste titre, et d'après de bonnes observations, que 
les anciens nommérent l'océan, le père de toutes choses.

Lt en effet est-il un marbre qui, à l'exception des os, des cufs des animaux, des arrètes de poissons, ne doive son existence aux mollusques et aux crustacés testacés, concurremmentavec les polypes madréporiques? et ne sont-ce point lí les deux agens principaux de toute la matiere calcaire? Qui ne connoit pas les marbres lumachelles ou coquilliers de la vallée d'Aoste et des autres côtes et vallées des Alpes, des Pyrénces, des monts Crapaks, des Cordillières? Qui n'a point admiré ces marbres de Ste-Anne et ces lumachelles de Namur, ainsi que ceux encoresi brillans de Carinthie, qui nonseulement disputent d'éclat, mais surpassent encore par leur iris et par leurs reflets la nacre la plus orientale et la plus pure; qui ont survécu aux àges, aux cocquilles les plus iriscies et les plus réflétantes? L'étude des roches et des pierres, des ossemens du globe, de ses parties solides, prend, nous le disons, sa source dans l'étude des coquilles et des mollusques qui les forment encore charue jour. Jamais on ne comnoitra l'origine du globe terraqué, sion ne lescludie à fond, et cette connoissance est enveloppée, est recelée dans l'étude des coquilles maintenant fossiles, ou abandonnées, autrefois mimćes, et dont la masse augmente chaque jour. Dans nos voyages, dans nos observations, d'accord avec dillustres naturalistes, nous avons saisi cette térité: c'est uné route dans laquelle on ne peut plus s'igarer.

Celle manière large et sûre, et ce point de vue sous lequel nous envisageons. les coquilles, du moment 
qu'elles sont abandomées par les êtres qui les formèrent, nous donnent des notions aussi certaines qu'exactes sur la théorie de la terre, et sur la formation du globe que nous habitons. Ce globe est né au centre des fluides aqueux, il est sorti du sein de l'océan; ses couches alternantes, dont les inférieures sont enveloppées par les supérieures, annoncent des générations successives, qui tour-à-tour ont payé à la nature le tribut d'une existence, dont elles fournirent le terme au sein des eaux. Ces eaux, à une époque des âges, recéloient le noyau de notre planète ; les continens s'y solidifioient, et chaque instant ajoutoit à leurs masses, destinées à percer un jour, et à surmonter les eaux qui protégeoient leur formation. Un terrible cataclisme bouleversa tout dans son incalculable puissance; la masse entière fléchit sur son axe, les eaux dans ce choc effroyable perdirent un instant leur ancien niveau pour en reprendre instantanément un autre. Voilà l'apparition de la terre maintenant asséchée; les collines, les montagnes, des chaines entières nées au sein des mers, se montrent à découvert; des mousses, des plantes, des arbustes les couvrent d'un tapis de verdure; la végétation terrestre se développe dans toute son énergie, les forèts se groupent et se confondent; elles se peuplent, et l'immense série des êtres du règne animal, née de cette époque, joint encore à chaque instant, et sous nos yeux, ses restes et ses débris à la masse commune, formés par ceux de tant de géné. rations.

Aussi, indiquerons-nous successivement, le plus qu'il nous sera possible, dans cette nomenclature des genres, 
ceux dont nous connoissons quelques analogues fossiles. Ces exemples seroient infiniment plus nombreux, si nous descendions a la description des espèces qui entre elles forment ces familles génériques ou congénères. Lorsque nous parlons de genres, nous entendons le faire d'une classe, d'un nombre quelconque d'ètres qui vienment se ranger sous des caracteres qui lcur sont communs, et dont tous portent le type, ou présentent lempreinte; la réunion de ces êtres, de ces espèces, forme donc un genre, quoique chacun d'eux présente encore, de plus que le type générique, un caractère qui lui soit propre. Ce caractère constitue l'espèce. Des différences de grandeur, de couleur, d'épaisseur, présentent les variétés, et on est convenu de ne point donner à cclles-ci la qualification d'espéces. Cependant nous devons observer que toutes ces divisions n'étant que de convention, elles devienment susceptibles de plus ou moins de modifications. D'accord arec les auteurs qui ont traité de lisistoire naturelle des coquilles, les oryctologues ou écrivains des fossiles ontadopté, pour terminaison des noms quils employerent, celle en lithe, du grec lithos, pierre, pour tout corps pétrifie: nous l'avons conservée partout ou nous n'avions a présenter pour type de genre quiune coquille pétrifiée, et dont on n'avoit pas encore rencontré l'analogue; dans le cas contraire, nous avons abandouné cette terminaison, et pour en citer un exemple remarcquable, nous avons donné le nom d'ammonie, à l'ammonite ou à la corne d'Ammon, dont, d'accord avecles faits et avec la vérité, nous avons présenté le type à l'état marin, à l'article qui concerne ces coquilles. 
Plus une science est cultivée, et plus elle acquiert d'étendue; et de fait, tous, tant que nous sommes, ne jouissons-nous pas des travaux de ceux qui nous précédèrent? Ne nous ont-ils pas laissé le point auquel ils parvinrent par leurs veilles et par leurs travaux? et dès-lors, ne nous imposèrent-ils pas le devoir d'ajouter, lorsque nous le pourrions, à leurs découvertes ? Chaque jour plus nous creusons les sujets, et plus nous voyons, surtout en raison des découvertes nouvelles, les objets se presser, s'entasser sous nos pinceaux, sous notre plume. Si nous ouvrons les livres des conchyliologues qui illustrèrent la science jusqu’à nous, à peine trouverons-nous douze genres établis pour la classification des coquilles chambrées ou cloisonnées. En approfondissant ces matières, en étudiant l'histoire de ces coquilles, et de celles qui ne sont pas cloisonnées, nous avons vu une nouvelle route s'ouvrir devant nous; nous avons été forcés même malgré nous de muliiplier de beaucoup ces genres; et cependant un mûr examen nous donne la certitude que notre travail sera accueilli.

Si nous envisageons d'abord les coquilles cloisonnées, nous verrons quà elles seules elles forment un ordre que nous n'avions pas encore aperçu dans la nature. L'ouvrage actuel prouvera combien leurs genres sont nombreux : il est même des coupes que nous ne voulûmes pas hasarder, mais qui indubitablement se feront naturellement dans la suite. Il devient nécessaire à l'égard des coquilles d'abandonner enfin une manière de voir, diamétralement contraire à l'ordre naturel, c'est-à-dire 
celle qui, regardant toutes les coquilles comme formées par des animaux congrineres, les range toutes sous une mème catrigoric : car les limaçons qui rampent comme les limaces sur le ventre, et que l'on nomme gastćropodes, ont une organisation bien différente des poulpes qui, de science certaine, forment les nautiles; des spirules et des anmonies, dont la tête est armée, et qui ne rampent plus sur le ventic. Ils forment donc un ordre à part, qui se revet des coquilles qui lui sont propres, et, d'apres son organisation, ces coquilles sont cloisonnées: il en est de droites, de turbinées, de spirales; clles adoptent enfin toutes les formes que nous retrouvons dans les coquilles univalres non-cloisonnées; parmi elìes, il en est de même d'isolées, et d'autres qui sont grouppées, qui vivent en société, ou solitaires; et les corquilles cloisonnées constituent done un ordre à part.

Il en sera de méme un jour des vers cylindriques, lorsque lion voudra comparer leur organisation a celle deces animaux, de ces mollusques, soit rampans sur le ventre, ou armés de bras sur la tète; et si lcur tèt est calcaire et coquillier, quelle n'est done point la différence qui existe dans leur organisation animale? Les anatifes, les balanes, les diademes, n'nut-ils pas plus d'analogie avec les crustacés, qu'avec les mollusques testacés ? et d'ailleurs, cette division n'est-elle pas en quelque facon établie, lorsque deux coupes aussi tranchées que celles des bivalves et des univalves, sont venues diviser tout l'ensemble ronchyliologique: coupes basées sur l'organisation des animaux des luvitres, des moules, des 
peignes, des cœurs; organisation si éloignće de celle des autres mollusques dont nous venons de parler, qu'elle paroit nous suffire pour en faire un ordre et des classes par elles-mêmes.

Il existe encore dans tous les systêmes de conchyliologie, une grande lacune qu'il falloit avoir le courage d'essayer de faire disparoître. Jusqu'à Soldani, les coquilles microscopiques avoient été presqu'entièrement négligées. Ces coquilles constituent non-seulement encore aujourd'hui la majeure partie des sables d'une foule de plages marines; mais elles forment aussi, pour ainsi dire, à elles seules, le fond d'une étendue immense de pays. Les flancs dénudés des montagnes calcaires en laissent quelquefois à découvert des bancs d'une grande étendue; elles couvrent dans leurs espèces si variées, par leurs couches nombreuses, épaisses et pressées, des climats tout entiers; l'ossature des Appennins et de quelques autres chaînes de montagnes, en est entièrement formé, et même une foule de bancs que jusqu'à ce jour on avoit regardés, d'après leur grain fin et serré, comme uniquement composés de matière calcaire solide, et qu'on appeloit même calcaires primitifs, sont entičrement coquillers. Il est vrai qu'au premier abord et à l'œil nu on croit n'y voir aucun vestige de coquilles, parce qu'on ne s'attend point d'y en rencontrer; mais elles y sont microscopiques; mais elles en forment la base, comme le découvrent tantôt les cassures et tantôt le poli ; mais on les y voit à l'aide d'une bonne loupe ; et elles se découvrent encore dès le premier aspect à ces regards percans comme ceux de l'aigle, partage du 
naturaliste, et ce quion appelle le coup-d'œil d'habitude. Le nombre de ces coquilles y est aussi pressé que celui des grains de sable des bords de la mer, ou comme nous venons de le dire, on les retrouve encore vivantes sur une multitude de plages. Ce n'est quà leur extrème ténuité quion peut imputer l'espéce d'oubli dans lequel on les laissa jusqu'à présent dans tous les systèmes conchyliologiques, et cela peut-être parce que, malgré qu'un œil exercé puisse les étudier à la loupe, il n’en est pas moins vrai que quelques-unes sont tellement exiguës, qu'elles veulent être soumises au microscope. Quant à nous, nous croyons être encore bien éloignés d'avoir donné tous leurs genres dans cet ouvrage ; mais au moins nous aurons fait comnoitre en partie leurs formes singulieres. Elles nous en offriront de si particulières qu'on pourroit quelquefois douter de ces formes, si leur conformation ne venoit en même temps résoudre une foule de problêmes, et nous prouver que toutes les places, malgréles lacunes qui existent encore pour nous, sont remplies, que la nature a modifié la matiere sous toutes lesformes, qu'elle l'a remanice sous tous les aspects; et que l'imagination même la plus féconde ne pourroit en créer une seule que la nature n'ait déja enfantée, et dont elle ne lui offre aussitót le type dans la graduation insensible, et dans la série immense des êtres procréés. Ces coquilles microscopiques qui existent dans une telle abondance, que Soldani a cru pouroir nommer terres nautiligues, les terreins qui leur appartiennent, sont loin de le cider pour leurs formes, pour leurs teintes et pour leurs robes, aux autres corpuilles qui, plus grandes, forment l'ornement 
de nos collections. Elles semblent même les surpasser eu égard à leur délicatesse et à leur ténuité; et nous savons aussi que jamais la nature n'est plus admirable que dans la construction et l'organisation des plus petits êtres, chez qui elle semble nous avoir voulu donner des preuves de l'immensité de ses moyens, dans le fini même le plus parfait. On doit donc au contraire, s'étonner que si peu d'auteurs aient entrepris leur histoire, dont tous, à l'exception de Soldani (I), se sont contentés d'en indiquer seulement quelques-unes: tels furent Bianchi, plus connu par le nom de Janus Plancus (2), chez les Italiens; Boys et Walker (3), chez les Anglois, et der-

(1) Saggio orittografieo ovvero osservazioni sopra le terre nautilitiche ed ammonitiche della Toscana, etc., dal Padre Don Ambrogio Soldani, ab. camaldol. in Sienna, $1780,4^{\circ}$. con tab. Cet onvrage traite uniquement des fossiles microscopiques.

Du même; testaceographiæ et 2oophytographix parvæ et microscopica, etc, Senis, 1789 et 179 folio. cum multis tabulis aneis.

(2) Jani Planci(Giovanni Bianchi ) Ariminensis, de conchis minus notis liber, etc., editio secunda. Roma, 176r. $4^{\circ}$ cum tabulis aneis.

(3) A collection of the minute and rare shells lately discorercd in the sand of the seashore near Sandwich; by William Boys, Esq. F. S. A. considerably augmented and all their figures accurately drawn and magnified with the microscope by Geo. Walker, Bookseller to Feversham. London , $4^{\circ}$. with plates. 
nierement von Fichtel et ron Moll (I), chez les Allemands. Bianchi en publia seulement quelyues especes, et cela d'une manière presque méconnoissable, acconpagnant son texte de figures tris-médiocres, pour ne point dire tout-a-fait mauvaises. Les deux auteurs anglois n'ont donné conjointement que les coquilles qu'on trouve dans les sables de Sandwich, en Angleterre, tt leurs descriptions ne remplissent pas entierement l'attente du lecteur. Quant aux écrivains allemands, ils sont minuticux, mais exacts, et quoique leur classification soit tris-imparfaite, leur ouvrage est excellent; d'ailleurs il nous paroit quils le regarderent cux-mémes comme un prodrome, comme un avant-conreur, et ils nous en ont fait espérer davantage. Soldani, de sou côté, écrivit sans méthode, sans caractériser les bouches de ses coquilles, et quoique son ouvrage soit bien propre à mettre sur la voie, cet auteur est bien loin d'avoir atteint le but; il eut à la vérité le rare mérite de donner beaucoup de coquilles, et surtout celles proprement microscopiques, tandis que les autres ne publièrent en général que des coquilles qui avoient presque une ligne et même plus de diametre; et on doit admirer cependant sa patience, et lui savoir gré de son travail, que lui-mème rendit si long et si minutieux, quoiqu'il n'ait embrassé que certains cantons particuliers.

(1) Testacea microscopica aliaque minuta ex generibus argonauta et nautilus ad naturam delineata et descripta a Lcopoldo von Fichtel et Jos. Carolo von Moll; cum 24 tabulis ceri incisis. Vindobonx, $1803,4^{\circ}$. 
Müller (I) à son tour décrivit, à la vérité, plusieurs coquilles microscopiques, mais il accompagna ses descriptions de bien peu de figures. Schrœter (2) en publia davantage, en ajoutant par le coloris, les teintes naturelles à l'égard de quelques-unes des coquilles qu'il décrivit ; et Spengler (3), de qui il sembloit qu'on devoit

(r) Von Würmern des süssen und salzigen Wassers, mit Kupfern, von Otto Frid. Müller. Kopenhagen I $7.7^{1} .4^{\circ}$.

Du même. Vermium terrestrium et fluviatilium seu animalium infusoriorum, helminthicorum et testaceorum, non marinorum, succincta historia. Havniæ et Lipsiæ, $177^{3}$ et $74.4^{\circ}$,

Du même. Zoologiæ Daniæ prodromus ; sen animalium Daniæ et Norvegix indigenarum characteres, nomina et synonyma in primis popularium. Havnix, $\mathrm{I} 766.8^{\circ}$.

(2) Die Geschichte der Flussconchylien mit vorziiglicher Riicksicht auf diejenigen, welche in den thüringischen Wassern leben, von Joh. Sam. Schroeter. Halle, $1779 \cdot 4^{\circ}$. mit is Kupfertafeln.

Du même. Plusieurs mémoires répandus dans ses grands ouvrages.

(3) Lorenz Spengler, inspectoris musæi rer. nat. et art. reg. Dan. Havn. tres tabulæ æneæ, cum iconibus testaceorum partim rarissimorum. folio.

Du même. Prælectiones in actis reg. Dan, societatis scientiarum Havn. cum tabulis æneis.

Du méme. Les planches gravées pour le catalogue de ses testacées quíil prépare. 
en attendre le plus, s'est contenté d'en publier un trèspetit nombre, que cependant nous devons regarder comme trés-rares. Parmi les autres conchyliologues des coquilles microscopiques, tels que Lister ( 1 ), Duchesne (2), Adanson (3), da Costa (4), Batsch, (5), Chemnitz (6), nous voyons qu'en général ils en ont publié très-peu; Batsch lui-même, quoique peintre et graveur, n'ayant, pour ainsi dire, donné l'initiative que de six espèces.

Nous aurions pu étendre ce travail à tous les auteurs

(1) Mart. Lister historix seu synopsis conchyliorum. Londini et Oxonix, $\mathbf{x} 685,1696$ et 1770 , folio, tabulx æri incisx.

(2) Recueil des coquilles fluviatiles et terrestres, qui se trouvent aux environs de Paris, dessinées, gravées et enluminées d'après nature, par Duchesne, etc., suirant l'ordre que leur a donné M. Geoffroy. Paris , ( sans date) $.4^{\circ}$. trois planches.

(3) Histoire naturelle du Sénégal. Coquillages , par M. Adanson. Paris, $1757.4^{\circ}$. avec figures.

(4) Historia naturalis testaceorum Britannix, or the British conchyliology. c. à. d. Conchyliologie britannigue, etc., arec figures coloriées, par Em. Mendel da Costa, London, ${ }_{17} 77^{8} .4^{\circ}$.

(5) Sechs Kupfertafeln mit Conclyylien des Seesandes, gezeichnet und gestochen von A. J. G. C. Batseh. Jena, $179^{1} \cdot 4^{\circ}$.

(6) Nenes systematisches Conchyliencabinet, fortgesetzt durch Joh. Hieron. Chemnitz. 1xter, Band, mit nach der Natur gemahlten Kupfern. Nürnberg, $1786.4^{\circ}$. 
qui ont traité de la conchyliologie, mais c'eût été entrer dans une espèce d'inventaire, long, pénible, fastidieux que nous avons fait cependant, mais que nous avons voulu aussi épargner à nos lecteurs. Quant à Martini, dans les actes de la société de Dantzick, Gualtieri et Ledermuller, ces derniers auteurs se sont contentés, dans leurs ouvrages, d'ailleurs estimables, de copier les autres, et ce quils publièrent à cet égard mérite à peine d'entrer en ligne de compte.

Dès le commencement de ce discours, nous avons dit que nos caractères génériques étoient tracés le plus sévèrement, et en même temps le plus succinctement qu'il nous a été possible; et nous devons observer ici que lorsqu'on veut tracer ces caractères, qui sont destinés à servir de type à un genre, on doit toujours chercher pour individu une coquille qui soit arrivée à toute la maturité de l'âge. Dans ses progressions successives, dans ses âges différens, le têt des mollusques n'est point toujours le même; et s'il falloit, pour appuyer encore ce fait maintenant si connu, citer de nouveau des exemples, nous pourrions faire observer que beaucoup de jeunes volutes ressemblent dans leur premier âge à des fuseaux, dont plusieurs sont mamelonnés au sommet, et qu'une jeune porcelaine ( cyprea) ressemble plus alors à un cône qu'à la coquille dont elle offrira les formes arrondies et renflées, quand l'age les lui aura fait obtenir dans toute leur perfection. Il en est de même des strombes, que le temps fait épanouir en aile, ce qui leur donne un aspect si différent, si éloigné de celui du premier âge, qu'il est presque impossible de ne point 
les regarder comme constitnant une autre espice, et de ne point se tromper; à moins qu'une longue habitude basće sur de fréquentes observations, n'ait fixé les idées sur cet objet. D'autres genres de coquilles, et parmi eux on doit ranger les limaçons, ont jusqu’a une certaine époque le bord extérieur de leur bouche tranchant et dirigé dans le sens de la spirale de leur coquille; mais à cette époque, ils y forment un rebord, un bourrelet lorsque cette même coquille recoit son complément; et c'est ce que les amateurs expriment lorsqu'ils disent que cette coquille a fait sa bouche, que sa bouche est. complète. Ces diflérences et ces aspects disparates exigent de la part des concliyliologues une étude et une attention ties-particulières. Nous remarquerons encore queles mollusques testacés forment l'enroulement, l'aspect extérieur de leurs coquilles, non point par des déveluppemens partant de l'extérieur et émanés du corps de ces mollusques, continus, et qu'autrefois on qualifia d'intussusception, systène absurde que nous voyons se renouveler encore de temps en temps dans un siccle qu'on regarde cependant à juste titre comme trés-ćclairé ; mais ces mollusques forment leurs coquilles par l'exsudation successive qui transsude du collier de l'animal, collier qui dans l'état d'action, de développement, reborde la bouche de leurs enveloppes testacées.

Dis que les formes extirieures sont arrétées, comme le prouvent ces stries fines et constamment dans le sens de sa bouche, qu'on pent voir sur toutes les coquilles sans exception, et qui sont indépendantes des cótes et des cordons; dès que ces formes sont dessinies, la co- 
quille est rendue de jour en jour plus épaisse par juxtaposition, c'est-à-dire au moyen de mollécules animales, spathiques et calcaires toujours nouvelles, et toujours transsudantes de toutes les parties du corps de l'animal qui sont en contact avec elles. Le bord du manteau ou collier des mollusques, forme encore ordinairement la robe colorée, le drap extérieur si vif, et d'autrefois si varié, qui embellit les coquilles; mais l'intérieur de ces coquilles, leurs parties mattes ou nacrées sont secondairement formées par un enduit postérieur de particules nouvelles, exsudantes du corps et de ses parties qui sont renfermées dans lintérieur des coquilles. Indépendamment de ces aspects différens, dus à l'âge et au temps, beaucoup de mollusques ont encore des époques plus rapprochées, ou ce même aspect varie, et ces époques sont en général annuelles ou bis-annuelles.

A des intervalles périodiques que règlent les saisons, le devoir de la propagation se fait sentir chez les mollusques dans toute sa puissance; quelques espèces se recherchent, d'autres se suffisent à elles-mèmes; alors, chez beaucoup de mollusques, les organes générateurs s'enflent, s'étendent, se dilatent, et la bouche de la coquille ne pouvant plus les contenir, ces organes saillans se recouvrent d'une enveloppe calcaire adhérente à celle déjà existante, et qui dessine leurs formes; mais le calme succède à la saison des amours, et alors tout rentre dans l'état de tranquillité et de repos ordinaire; voilà l'origine de ces tubercules, de ces bourrelets intermittens, de ces cordons perpendiculaires au sommet, et 
de ces côtes épincuses et frisées qu'on voit sur tant de coquilles, et principalement sur les casques et sur une toule de pourpres et de chicoracées. La coquille est-elle unie? c'est l'indication du temps de repos: montre-t-elle un bourrelet ou des épines, c'est l'indice de l'intermittence d'un temps d'activité qui lui succéda périodiquement ; et ces protubérances sont feuillées ou ćpineuses, d'apres la forme des organes alors en pleine extension, sur lesquels elles se sont moulćes. Quant aux coquilles bivalves, leurs mollusques sont attachés au tèt par un ou par plusieurs muscles qui ne se déplacent jamais, et qui, en augmentant d'annće en année, forment à fur et mesure de plus grandes impressions dans linterieur des valves; tandis que les mêmes époques d'une vie plus active sont marquées sur le dos des valyes par des cordons plus ou moins renllés et paralliles aux sommets de ces mémes valves; et chez tous les mollusques testacés on peut présumer leur âge par le nombre de ces saillies ou cordons, qui semble, par leur moyen, s'ètre inscrit en caractires ineflaçables presque tonjours sur les parties extérieures des coquilles, et quelquefois dans leur intérieur, comme nous le prouverons successivement quand l'occasion nous en prísentera des exemples.

On trouvera aussi dans cet ouvrage les figures des coquilles que nous avons dessinées, placées non point dune maniere pittoresque et proprea faire eflet, comme (.lles le sont dans tous les autres ourrages de conchyliologie, mais montrant leurs caractieres: c'est encore un sacrifice que nous arons été forcés de faire, afin de rendre le plus correct quiil nous a été possible, un ou- 
vrage élémentaire et didactique. On y verra en conséquence les coquilles univalves toujours présentées de manière à ce qu'elles offrent leur bouche vers le bas de la planche et leur spire élevée; position ingrate pour le dessinateur, mais de laquelle tous les auteurs qui écrivent maintenant sur les coquilles, sont enfin convenus, afin de s'entendre lorsqu'ils parlent de la base d'une coquille, de son sommet, de sa lèvre gauche ou de sa levre droite. Quant aux coquilles bivalves, dont nous traiterons dans notre troisième volume; comme leurs caractères génériques sont pris principalement dans la considération de leurs charnières et des dents qui les composent, nous avons préféré de multiplier les figures en faveur de la clarté que nous recherchons par-dessus toutes choses, et de consacrer particulièrement un dessin à ces mêmes charnières lorsque le cas l'exigera. C'est pour ces raisons que lorsque nous traiterons de cette classe de mollusques dans notre troisieme yolume, qui formera le complément de notre conchyliologie systématique, nous donnerons d'abord la figure de la coquille servant de type à chaque genre, telle qu'elle existe pendant la vie de l'animal à qui elle sert de revêtement, et nous indiquerons la structure des charnières par des figures destinées uniquement à cet usage, ainsi que l'ont dẹjà fait quelques auteurs anglois.

A l'exception peut-être des mollusques testacés qui par leurs formes et leur organisation, viennent, comme les nautiles et les spirules, se rattacher aux poulpes, aux sèches et aux calmars, tous les autres mollusques testacés réunissent dans le même individu les deux sexes; 
aussi loutes les coquilles d'une même espéce, recueillies sur lia mème plage, ne présentent aucune différence, à moins quion ne venille regarder comme telles desimples accidens. On ne peut donc point, ainsi que l'ont fait quelques anciens auteurs, distinguer les coquilles comme ayant appartenu à des máles ou à des femelles, en raison de la vivacité de leurs couleurs; prejugé qui existe encore et principalement pour les huitres, que lespécheurs qualifient de máles, lorsque leurs mollusques ont les barbes ou branchies noires. Trois modes de grénciation senblent être l'apanage accordé par la nature aux mollusques testacés; chez les uns, chez ceux qui se rapprochent des sèches et des poulpes, les sexes sont scparris, et cette scission a lieu de la manière la plus complète; là le concours de deux individus de sexe différent est impérieusement exigé pour donner naissance à un troisième être qui, uniquement mâle ou femelle, propagera la race aux mêmes conditions. La fenselle porte l'ovaire, et le mâle seul posséde l'aure séminale. Chez d'autres mollusques testacés l'animal renferme en lui les organes des deux sexes, et tellement placés, que l'organe mâle puisse féconder l'organe fémelle sans le concours d'aucun autre individu. C'est ici l'androgyneité dans toute son intrigrité primitive. Les genres des animaux qui jouissent de ce morle de propagalion sont extrêmement nombreux. Non-seulement beaucoup de bivalves se rangent parmi cux, mais encore des multivalves, des rissiralves, et méme quelques univalves : tous ces mollusques muliplient dans lisolement, renlermés sous leur têt et dans la solitude la plus profonile;et siquel fues-uns d'entr'eux recourrent en masses 
et très-souvent de très-grands espaces; si, comme les huîtres et d'autres coquilles adhérentes, ils masquent des surfaces tout entières de rochers, il n'en est pas moins vrai qu'ils ignorent l'existence de ceux de leurs congénères qui se trouvent leurs plus proches voisins. Enfin le troisième mode de propagation est celui des hermaphrodites, celui ou ces mollusques, quoique pourvus des deux sexes, ne peuvent se féconder euxmêmes, mais doivent chercher, rencontrer un autre individu de leur espèce, qui, comme eux, soit stimulé par le besoin de la reproduction. Combien leurs recherches et leurs amours ne sont-ils pas variées? Tantôt ce sont deux êtres qui s'isolent du reste de la nature, qui se provoquent par de douces caresses, qui se stimulent par des agacerics mutuelles; qui, comme chez les limaces, et parmi les limaçons, se dardent des flèches amoureuses, acérées, aiguisées au flambeau de l'hymen, finissent par s'étreindre, par s'enlacer, et par se plonger mutuellement, par une doublejouissance, dans l'excès d'un long bonheur. Tantòt c'est un peuple tout entier qui, comme chez les planorbes, travaille en masse à la propagation de l'espèce : chaque individu placé dans son rang reçoit d'un côté ce qu'il prête de l'autre ; et c'est à ces époques de multiplication qu'on retire du fond des eaux douces ces longs chapelets de planorbes, qui tapissent au printemps les flancs des fossés. Dans l'ouvrage actuel nous ne nous étendrons point davantage sur ces observations; il est destiné à la classification des coquilles par leurs propres caractères, sans rien emprunter à ceux des animaux, objet que cependant nous pourrons développer un jour. 
Cherchant done à traiter la conchyliologie proprement dite, dans l'étendue qui lui est propre, nous avons été forcés, d'après les découvertes nouvelles et nos propres olservations, de créer une foule de genres nouveaux, dont quelques-uns avoient dejà, comme nous l'arons dit, été soupconnés et mème indiqués par d'autres conchyliologues. Nous avouons avec franchise que rien ne nous a autant causé d'embarras que les noms que nous avons été dans le cas de leur imposer. D'un cóté nous n'avons point cru devoir sacrifier à la manie moderne, qui consiste à tirer une dénomination de la langue grecque, en la faisant dériver soit de laspect, de la forme, de la couleur, ou de tout autre carac lére de l'objet que lon reut décrire. Rien d'ailleurs ne paroit olfrir autant de prise à la critique qu'un nom significatif el indicatif tout en mème temps de la chose elle-mène, et ces noms ne furent que trop souvent une pierre d'achoppenent contre laquelle vinrent se briser les meilleurs idées, comme nous le prouve linterminable querelle devenue à plus d'un égard une dispute de mots, sur la nomenclature, entre les chimistes de lancienne école ct ceux de la moderne. Les noms insignifians sont, it ce que nous croyons, préférables en eux-mémes, parce que ne se rattachant à aucune idée complexe, ils ne laissent sous ce rapport aucune prise, et c'est toujours autant d'érité. Mais, d'un autre cúté, on veutarec juste raison quils soient aimables et sonores; on reut quils remplissent l'oreille agréablement, et quils ne soient ni trop courts, ni trop longs, sans syllabes dures on barbares; et les hommes laborieux yui ont passé par les memes épreures, savent quel embarras, quion auroit 
cependant peine à croire réel, on éprouve à cet égard. Tantôt nous avons puisé ces noms dans ceux des divinités et des nymphes de la mer et des eaux dont, pour ce sujet, on n'avoit jusqu’à présent fait encore aucun usage; mais comme cette mine a été très-fouillée depuis quelque temps, elle n'a pu nous fournir ce que nous en avions espéré; d'autrefois, par conséquent, nos choix aurontété moins heureux, et nous avouons ingénûment que nous avons, à cet égard, besoin de la plus grande indulgence. D'ailleurs nous abandonnons ces noms de bonne grace, en désirant même que tous ceux à qui ils pourroient déplaire veuillent bien les changer. Du reste nous avons adopté par devoir tous ceux qu'employèrent les conchyliologues systématiques qui nous ont précédé dans la détermination de leurs genres; heureux de pouvoir nous approprier un semblable travail, quoique même très-souvent les genres que nous établissons ne soient plus intégralement les mêmes que ceux indiqués par ces illustres devanciers.

Nous espérons que sous tous les rapports nos lecteurs voudront bien regarder cet ouvrage comme original, comme fruit de nos travaux, et comme nous appartenant en propre; en le distinguant de ces compilations indigestes qui ne coûtent à ceux qui les mettent au jour d'autre peine que celle de s'emparer des travaux d'autrui, et de copier servilement ce qui a été dit avant eux, sans rendre aucun hornmage aux auteurs qui, à juste titre, pourroient leur reprocher leurs plagiats. Souvent, et nous l'avouons encore avec reconnoissance, nous avons joui des travaux de ceux qui écrivirent avant 
nous, et c'est leur rendre l'hommage le plus digne d'eux, parce quil renterme l'aveu tacite que nous n'avons point pu mieux faire: mais les recherches qui nous sont personnelles, nos autres ouvrages, les olservations qui nous appartiennent, les longues études que nous ayons faites sur les matieres que nous traitons, et les royagres auxquels nous nous sommes livrés dans le scul espoir d'ajouter de nourelles découvertes à tout ce qui étoit dëja connu, prouveront incontestablement en notre faveur. D'ailleurs, depuis le temps que nous étudions les coquilles, soit par nos travaux, soit par des observations verbales, nous avons émis pliss diun fait, plus d'une idcé, dont les uns se sont simplement emparés, et qui germerent chez d'autres; et en conséquence nous aurions bien aussi quelques réclamations à faire; mais enfin aujourd'hui nous publions la masse de nos idies sur cette partie si brillante de l'histoire naturelle, et nous reprenons sans rancune tout ce que nous croyons pouvoir légitimenent réclamer. C'est par suite de ces observations et de ces recherches que mus par la vue de l'ensemble du régne conchyliologique, nous avons $\mathrm{cru}$ devoir adopter une division nouvelle, ou ajouter à celle deja reçue quelques coupes. Dans ces vues nous avous divisé l'ordre entier des coquilles en univalves, dissivalves, multivalves et bivalves, en adoptant quelques subdivisions pour les coquilles cloisonnces, spirées ou non cloisonnées, à bouches échancrées ou à bouches non-échancrées.

L'acception des dénominations d'univalves, de multivalves et debivalves est assez connue, pour que nous 
regardions comme inutile de la développer ici ; d'autant plus que nous renvoyons à la terminologie conchyliologique que renferme cet ouvrage : mais il n'en est pas de même de celle de dissivalves, dont nous nous servons les premiers, et sur laquelle par conséquent nous devons quelques explications.

C'est en considérant attentivement la série des coquilles qu'on est convenu de regarder comme multivalves, que nous nous sommes aperçus qu'elles devoient nécessairement être divisées en deux classes, division présentée par la nature elle-mème. De ces coquilles, les unes sont effectivement multivalves, toutes les pièces qui forment par leur réunion l'ensemble de leur têt, étant plus ou moins mobiles et liées entre elles par des charnières, des ligamens ou des nerfs qui les réunissent assez intimément pour ne point laisser de solution de continuité entre elles; telles sont les anatifes, les pholades, etc. Mais il en est d'autres, ainsi que les tarets, les fistulanes, les balanes, les coronules, les tubicinelles, etc., qui à la vérité présentent bien plusieurs pièces testacées, mais non cohérentes ni adhérentes les unes aux autres; elles sont sans nerf ou ligament commun, et totalement disjointes de la pièce principale:les tarets logés dans un tube testacé ou coquillier, ont de plus deux valves qui en sont isolées et placées sur une partie du corps du mollusque, qui a de plus deux spatules de semblable substance à la partic antérieure de son corps, indépendantes de son tube testacé, et qui ne lui adhèrent en aucune manière : il en est de même des fistulanes dont le corps renfermé 
dans un semblable tube, recele deux petites valves que rien n'y rattacle: quant aux quatre valves qui couronnent le sommet des balanes, des coronules, des tubicinelles, elles tiennent au corps de leurs animaux, et point du tout à la valve principale; raison pour laquelle ces coquilles qu'on voit dans les cabinets en sont ordinairement dépourvues. Il est très-probable que l'arrosoir et peut-être quelques autres corquilles encore, viennent se ranger dans cette classe, dejjà assez nombreuse parce qu'elle renferme tous les balanes marins et pétrifiés dont les espèces sont plus multiplices qu'on ne le croit communément; c'est à ces coquilles que nous avons cru pouvoir appliquer la qualification de dissivalues, équivalent de values dissidentes, et mot en harmonic avec ceux déjà consacrés d'univalves, multivalves et bivalves.

Celte division nous a paru simple. Il est rai qu'il semble en exister une autre, qui seroit peut-itre plus conforme encore à l'ordre naturel, basée sur l'organisation des animaux, que nous avons été tentés de suivre un instant, et que nous indiquons ici à ceux qui voudront s'en servir un jour lorsque les animaux des coquilles seront enfin plus géníralement connus. C'est en nous répétant en quelque façon que nous observerons à nos lecteurs que certains mollusques testacés, tels que les habitans de beaucoup de coquilles cloisomées, et les argonautes parmi celles non cloisonnées, viennent se ranger à côté des séches et des poulpes; d'autres, tels que ceux des cones, des volutes, des helix, à cóté des limaces; d'autres, tels que les serpules, les siliquaires, 
lestarets, à côté des vers; d'autres, tels que les arrosoirs, à côté des polypes; d'autres, tels que les balanes, les lingules; les anatifes, à côté des crustacés; d'autres, comme les camérines, les rotalites, à côté des vélelles et des méduses; et qu'il en est de même de toutes les coquilles dont la nature a décoré des êtres chez qui elle avoit déjà successivement épuisé toutes les formes du nu. 


\section{TER MES}

USITÉS EN CONGHYLIOLOGIE,

O U

VOGABULAAIRE

CONCHYLIOLOGIQ UE

ET RAISONNE

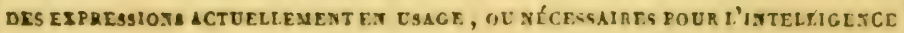
DE CETTE BRASCHE DE L'HSTOHE NATURELE.

A.

МBattras, ABATtu : se dic de certaines pointes on feuillelis que plusients mollusques abattent annuellement de la surface de leurs coquilles : beauconp de murex, ies bécasses épineuses et les chicoracées sont dans ce cas.

ABDOMEN. Partie postérieure et inférienre du corps de quelques mollusques. Le ventre.

ABDOMINAL. Da ventre.

ACCOLLÉ. Juint, réuni deux à deux; les valses des bivalves sont accolées.

ACEPHALE. Dénomination comprsée de l'a gree privatif, et de kephalè, tête. Sans sête. Le mollusyque acćphale ès donc un mollısque sans têtc. 
TERMES USITÉS FN CONCHYLIOLOGIE. XIVi]

ACÉPHALE. Signifie la même close.

ADHÉRENT. Beaucoup de mollusques adhèrent à leur coquille.

Adhérent. Une coquille qui s'attache est adhérente; les huîtres le sont ans rocliers.

Adhérent. Un opercule est adhérent lorsquili tient à l'animal.

AILE. Bord ou lèrre extérieure d'une coquille fort épanonie. Quelqunes strombes s'épanouissent en aile.

Aile. Le manteau des mollnsques des porcelaines recouvre dans son ampleur la coquille par deux ailes. Leur manteau a deux ailes.

\section{AMPLE. Large, étendn. \\ AMPLEUR. Iargeur.}

ANALOGUE. Semblable, pareil, identique ; on trouve parmi les coquilles pétrifiées de fréquens analogrues de celles qui vivent encore aujourd'hui dans les mers.

ANATIFERE. Porte-canards. Nom ancien, et propageant une crreur prossière, improprement donné à l'anatife.

ANGULEUX. Une coquille, au lieu d'ètre arrondie, peut être angulense. On voit encore des dents angruleuses.

ANUS. Ouverture, sortie des excrémens.

APPENDICE. Partie pendante, excédeate, ordinairement charnue, et allongée en filet.

APLATI. Qui est plat; une coquille, au lien d'être ronde, pent être aplatic.

Aplati. Lorsqu'une spire est écrasée, an lien d'être élerée , elle peut être aplatie.

Aplati. Cette épithète se donne encore anx spires renfoncées des coquilles en orbes on en disques. La spire des planorbes est aplatio.

ARÊTE. Partie saillante, allongée ct trèq-marquéc. Les hippurites ont deux arrêtes intérieures et caractéristiques.

Arête, à vìve arếte. Coupé, tranché net; en lame de contean.

ARMATURE, ARMURE.

ARMATION, ARMURE, ARMÉ. Une coquille est armée 
iorsyu'elle est chargéc d'épines; elle porte sun armature ou armure.

AROUÉ. Dessiné en are; se rejetant en arriere. Certaines coquilles ont un bec arqué.

ARRONIJI. Fait en rond.

ARTICULATION. Insertion, suture et mêne partic intêgrale d'une coquille. Des cormes d'Ammon sout formées par une saite très-noinbrense d'articulations.

Arliculation. Les tentacules des balanes, ou glands, ont des articulations.

Articulation. On dit encore l'articulation des charnières en parłant de l'engrènement de leurs dents: quelyues bivalves ont lenr charnière articuléc.

ARTICULÉ. Qui a des articulations, on qui est formé par elles.

ATT $\triangle$ CHE. Piece calcaire indépendante des ralres de la coquille; les anomies ont une attache, ou troisième piece qui les fixe aux luitres et aux rochers.

AURICULE. Qui a des oreilles. Des peignes sunt aurienlés.

AURIFORME. Portant la forme, fait en forme d'orcille.

AUSTRAL.Du sud, renant du sud, appartenant au sud,

AXE. Contre d'unc coquille. L'axe des nautiles, des plaurblos les traverse d'un flanc à l'autre: l'axe d'un cadran, d'une pyra. midelle descend du sommet jusqüà la base.

\section{B.}

BASE. Partic d'en bas d'une coyuille lorsque la pointe de sa spire regarde le ciel, et par conséquent celle opposice an sommet, ou pointe de la spire. Les volutessont chancrées a lenr base.

Base. On donne encore ce nom d̀ la partie basse; chez les mollusques les yeux sont quelquefois placés à la base des entacules.

Zase. Pied. Les lepas reposent sur les rochers par leur base.

Buse. Pied. Les balanes sont adlérens par leur base ; les diadêmes le sont de même sur le dos des baleines. 
Base. Linné donna encore ce nom anx sommets des coquilles bivalves, ou crochets, parce que, pour décrire ces coquilles, illes posoit sur les sommets, le ligament tourné vers le spectateur.

BAILLANT. Ouvert, sans pouvoir se fermer; il se dit des bivalves. Les manches de couteaux sont baillans à leurs deux extrémités: la mye, à une seule.

BEC. Base allonģée de certaines espèces de coquilles univalres. Les bécasses épineuses, les massnes d'Hercule, le murex braudaris, quelques fuseaux ont un bectrès-allongé.

Bec. Extrémité allongée des valves dans quelques bivalves. Certaines tellines ont leur partie ant érieure prolongée en bec.

Bec. Les poulpes, calmars et seches ont la bouche garnic de deux mâchoires cornées faites en bec de perroquet.

BIFIDE. Double, séparé dans son milieu et en longueur. Dent bifide, dent donble.

BOMBÉ. Élevé en bosse, arrondi, fait en bombe. Les porcelaines, les tonnes sont bombées.

BORD. Extrémité d'une coquille, rang extéricur. Bord d'une patelle, d'une fissurelle.

Bord. La lèrre extérieure se nomme encore quelquefois bord. La lèrre droite, le bord droit des ptérocères, des lambis, des strombes, s'élargit on s'épaissit arec l'àge.

Bord. Les valves des coquilles biralves ont leurs bords, tantôt unis, tantôt crénelés ou festonnés.

Bord. On dit aussi le bord du pied, du disque, du mantean; ces bords sont quelquefois frangés.

BOREAL. Qui vient du nord, appartenant au nord.

BOUCHE. Ouverture arrondie, allongéc et quelquefois même en triangle et qradrangulaire; elle est formée par le dernier tour de spire: c'est la bouche des coquilles, mais non des animanx qui y sont renfermés. C'est de la bonche des coquilles que la conchyliologie emprunte principalement ses caractères pour la classification systématique des coquilles univalres et dissivalves. Sonvent elle se divise en deux lèvres, l'une droite et l'autre gauche, et dans d'autres espèces la bouche est parfaitement circulaire et continue. Afin de s'entendre unavimement, les conchyliologues 
sont convenus de placerles couguilics de manière à ce que la bonche se présente de face et par en bas : c'est dans cette situation qu'ils en font la description, et quils en analysent les caractères.

Bouche se dit encore de la bouche réelle de l'animal, de l'ouverture quilui sert à saisir sa nourriture, et qui conduit à l'asophage; la tète des limaçons est garnic d'une bouche, celle des huitres est an milieu du corps.

BOLRRELET. Espée de cordon, saillant, arrondi ct prolongé qui borde la bouche de quelques coquilles; quelques limagno acquièrent ce bourrelet arec l'àge.

Bourrelet. On donne aussi ce nom aux cordons, restes d'une ancienne bouche, et qui ornent certaines coquilles; ces bourrelets sont this-apparens dans les culottes de Suisse.

BOUT. Extrémité sailiante d'une coqquille dont les denx lères sont roulées et volutées, et dont les bouts s'allongent, comme dans les navettes.

BRANCIIES. Espèces de bras plus ou moins contonrnés, et queliquelois osseux, qu'on trouve chez quelques mollusques et dans l'intérieur des ralves qui les recouvrent: les poulettes, anomies, ou térébratules sont munies de branches semblables, et elles sont ossenses.

BRA NCHIAL. Appartenant aux branchics on à leur roisinage. Ailes branchiales,

BRANCHIES. Organes respiratoires, et qui serrent de ponmous à beancoup de mollusques. Au moyeu des pores absorbans dont les branchies sont munies, cesanimaux séparent l'air propre à leur existence de l'air atmosphérique ou de l'ean qu'ils aspirent, et ils s'en approprient les particules nécessaires à la prolongation de leurvie.

BRAS. La partie antéricure du corps du mollusque de la lingule cst garnie de deux bras ciliés, que l'animal fait rentrer en les roulant en spirale : ceux de l'orbicule sont frangés ; ils s'allongent et se roulent de même.

BULLEUX. Arrondi, mais léger, mince, transparent, en bulle.

BYSSUS. Espèce de soie, de fil formé par yquelques mollusques 
bivalves; les monles s'attachent par un bissus; celui des pinnes marines se laisse filer, et on en fait des bas qui égalent ceux de soic.

\section{C.}

CAL. Renflement épais, solide et placé en surcharge, qui recouvre l'ombilic ou les lèvres de quelques coquilles. La columelle de l'hélicine a un cal:

CALLEUX. Chargé d'un cal.

CALLOSITÉ. Même signification que Cal. Beancoup de bivalves présentent des callosités dans leur intérieur.

CALCAIRE. Matière calcaire, matière propre à faire de la chaux. Les marbres, les craies sont des matières calcaires; et comme les coquilles, lorsqu'on les brîle, se convertissent en chanx, elles sont formées de matière calcaire.

CANAL. Plusieurs genres de coquilles dont la bonche est échancrée, ont un canal à leur bass; c'est une espèce de bec, plus ou moins court, tantôt droit, tantôt recourbé et formé en gouttière. Les cérites ont un canal recourbé, celui des strombes est court, dans les pleurotomes il est droit.

Canal; se dit encore lorsque, dans une spire, les tours sont séparés par une espèce de petit canal ou rainure qui règne dans tout leur prolongement. Les olives sont remarquables parce qu'elles ont constamment un canal à leur spire.

CANALICULÉ. Pourva, muni d'un canal soit à la base, soit à la spire. Fait en forme de canal.

CAPUCHON. Lorsqu'il est crochu ou recourbé, le sonmet des patelles forme le capuchon.

CARDINAL. Principal, très-remarquable, très-saillant. Quel. ques conchyliologues ont appliqqué ce mot aux maîtresses dents, aux dents principales des charnières dans quelques bivalves. Daus la charnièreà quatre dents des bucardes, deux sont cardinales, les mactres n'en ont qu'une.

CARÈNE. Fond, quille d'nn vaissean; les coquilles faites en vaisseau, en bateau, ont une carène. Les argonaules on nautiles papiracés sont carénés. 
CARÉNL. Formé en carène.

CARLE, rongé, crensé; le lutime thiarre et quelques coquilles uniralves lluviatiles ont leur sommet carié.

CARINLE. Même signification que caréné.

CARNIVGlí. Qui dérore, qui mange de la chair: l'animal qui sen nonrit est ua animal carnivore: it y a des mollusugues carnivores.

CARTILA GE. Partie solide, moins dure que la corne, ę plus ferme que la chair.

CARTILAGINEUX. De substance cartibangnese : formé en cartilage.

CAUDÉ. Du lasin cauda, quene. Garni, muni d'une queue.

C.IVI'Jí. Cremx d'une conquille. Le corps d'un limaçon ene contenu dans la canité de son tét. La carité est lintérienr de la spire, et elle est terminée par la bouche de la corguille.

CEILULE. Connille cellulée, renfermant une foule de petites chambres, de cellules. Les camérines sont des coquilles cullulées.

CHEVRON. Sunche fite en chevron, en équerre.

CENThE. Milien. La spire des ammonites commence au centre du disque formé par les rérolutionis de cette spire. Les cadrals ont un ombilic au centre de leur base.

CENTKAL, au cetate, ducentre ; appartenant au milicu, an centre.

CÉPIIALE, mot grec francisé: il signifie ayant une LEte. I.e limaçon est un mollusque céphalé.

CEPIALOPODE. Pieds sur la tète. Crtte familfe de mollniques oflie plusieurs genres : les seches, les calmars ont les pieds on bras sur la tête; ils sont céphalopodes.

CERCLL, còte grosse et transversale qui semble cercler qquelques coquilles; les lannes, liz peralrizi sout cerclées.

CERCLE, chargé de côtes cn traver's.

СНАMBRE, espace renfermie entre denx cloisms on pieces intéricures dans la casicé d'une conquile. Partie siparéce dans la cavité d'une coquille. Un orlinocénatice at des chambres.

CHAMBPE, coqnille gui a des chambres, yui est chambré. 
CHARNIERE. L'endroit du dos sous les sommets, par lequel les goquilles bivalves et multivalves joignent leurs battans ou valyes. Ordiuarement les charnières sont dentées, et quelquefois unies; et c'est de leurs dents, deleur position et de leur absence qu'on est convenu de tirer les principaux caractères propres à diviser les bivalves en genres respectifs. I.es charnières sont intéricures, clles se joignent et se recourrent l'une l'autre; et les dents dont elles sont armées rentrent aussi les unes dans les autres. La charnière des huîtres est sans dents, colle des vénus en est garnic; elles sont très-multipliées dans celle des arches.

CHARvU. De chair ; mou, ni ossenx, ni corné, ni cartilagineux; beaucoup de mollusques ont un pied, un corps, un mantean charnus.

CHICORACÉ. Frisé, formé en feuilles de chicorée. Plusicurs nutrex ont les bourrelets ou cordons garnis de fcuillures chicoracées.

CIL. Poil court et un pen recourbé, assez fort ct assez semblable à cenx qui dans l'homme bordent les paupières. Pinsicurs mollusques ont un manteau bordé de poils, un manteau cilié.

CILIÉ Garni, bordé de poils courts, de cils.

CIRCONVOLUTION. Tour entier de spire, révolution tonté entière; dans les nautiles le dernier tour de spire embrasse tous les antres par circonvolution.

CIRCULAIRE. Fait en cercle, se dossinant en cercle, en rondeur.

CIRCULAIREMENT. Disposé en cercle.

CLOISON. Pièce intérieure, parois disposées en travers dans la cavité, dans le creux d'une couguille, et la séparant, la divisant en concamérations on en chambres. Les nauliles, les cornes d'Ammon ; sont des coquilles cloisonnées.

CLOISONNÉ, qui a des cloisons.

LLOS : feriné, sans laisser d'ouvertures; il se dit principalement des bivalves. I.es ralves du manche de couteau cont baillantes, celles de la moule sont closes.

Clos, fermé, clos ; certains mollusques bivalves closent la bouche de leurs coquilles avec un opercule. 
Clos. Les limaģons closent leurs coquilles pendant l'hiver.

COLUMELLE, lerre intéricure des coquilles univalres, celle (qui forme le style on centre de la coquille; elle est lisse, callense ou arméte de dents, et c'est d'après ces différens caracteres qu'on emprunte les plus décidés de ceux qui serrent à classer ces coyuilles. La columelle des cónes est lisse, celle des mitrés est cientie, les anaulaces ont une columelle calleuse.

COMPRIMÉ, aplati, presque écrasé. Des plis, des dents penvent être comprimés. Les vasuliches sont des coquilles comprimées.

CUNCAMÉRATION. Cliambre formée par des cloisons placées dans la cavité des coqquillea polythalames ou clambrées. On dit les concanérations d'un nautile, celles d'un lituite, d'un orllocératilhe, d'un hippurile.

CONCAMERÉ, ayant des concamérations ; coquille chambrée, cloisonnée.

CONCAVE, qui est creur, l'opposé de convexe.

COYCHYLIVELE; chargé d'une coquille, portant un têt coquillier. Le's mollusques testacés sont concliylifères.

CONCHYLIFORME : façonné en forme de coquille, semblable à une coquille.

CONCHYLIOLOGIE, connoissance, science des coquilles.

CONCHYLIOLOGUE : celui qui conuoît les coquilles, l’auteur qui en traite; qui parle, qui écrit sur les coquilles.

CONCRETTION, converture étrangère; quelques coqquilles sont recouvertes de concrétions, soit de plantes ou de craie, qui lcur sont étrangères.

CONE. Fignure en còne, en pain de sucre. La flamboyante est une coyuille formée en cône.

CONIQUE. Fait en còne, en pain de sucre.

CONXÉ. Soudé, collí l'an ha l'autre, l'un sur l'autre, de manicre à ne sembler former qu'un mème corps. Les valves de l'hiale sont connces.

CONGEVERE. De mềe genre; tontes les espèces de harpes sonit congénères.

CONOIDE. Fait en cône aplati. 
CONTIGU, voisin, très-rapproché; quelques harpes ont leurs côtes contiguës; les deats de la charnière des arches sont sériales et contiguës.

CONTOUR. Surface extérieure et toute entière d'une coquille; les timbales, les tonnes sont arrondies dans leur contour: celui des murex est anguleux et rompu.

CONTOURNÉ, courbé en rond, formant le contonr, contourné en spirale, en sphère, en globe. Le spirule est contourné en spirale, le girogonite en sphère.

CONTRACTÉ. Replié, serré sur lui-même, rentré. Le limaçon p:ut à volonté contracter le pied sur lequel il rampe.

CONTRACTILE. Qui est susceptible de se contracter.

CONVERGENT. Accollé, courant dans le même sens, dans la nême direction. Les deux arrêtes intérieures de l'hippurite sont convergentes.

CONVEXE : relevé en bosse, en dos d'âne. Les lépas, les cabachons sont des coquilles convexes.

COQUILLE. Corps testacé et calcaire qui recourre les mollusques univalves, bivalves, multivalves et dissivalves; il $\mathbf{y}$ a cependant quelques coquilles ou pièces testacées intérieures et renfermées dans le corps de l'animal, telles sont celles des bullées, et les valves intéricures des fistulanes: l'hiale, la camérine sont encore des coquilles intéricures.

COQUILLIER. Formé d'une substance calcaire, de la même matière que les coquilles.

CORCELET. Partic exposée aux regards de l'observateur, lorsqu'une coquille biralve est conchée sur ses erochets. Linné employa, pour la désigner, le mot de vulva, repoussé justement par la langue françoise. C'est la partie qui porte le ligament; elle est très-remarquable dans les vénus. L'écusson est dans le milieu du corcelet, qui est armé dans la conque de Vénus.

CORDIFORME. Fait en forme de coeur. Par la réunion de leurs valves, beaucoup de bivalves sont cordiformes.

CORDON. Strie transversale, allongcee et colorée, quelquefois tuberculée et pointillée, proéminente sur le dos d'une coquille. Le cône amiral grenu, celui amiral de Curaçao ont des cordons. 
C(ORI)() V V É. Ciharené de cordons, qui a des cordons.

CORIA(:E. Dur, fibrenx, se déchirant dificilement; tenant le milien entre la como et la pran. Quelyues limaces ont entre cuir et chair us éeusson coriace.

CORIACE : qui est coriace.

COR VE. Fait de matiere analogue à la corne, ainsi que le son! beaucoup d'operenles dans les mollusques univalves, et les ned ou attaches dans les bivalves.

CORNET. Coyuille en cornet, rouléc, faite en cornet de papier.

COSTÉ, qui a des côtes. Garni de côtes.

COTES. Stries allongées, renflées, protubérantes et relevies, se dessimant perpendiculairement sur les conuilles. Les cites de la harpe nuble sont plus espacées que celles du manteau de Si.James; cet celles du coeur de boeuf épineux et de la conjue exotique sont triangulaires.

COTONNEUX, velu, velnuté; quelques coquilles univalves ont le drap marin cotouneur.

COUDE. Courbure subite et presque rompue; les cćrilhes ont un canal en coude.

COUDÉ. Fait en coude, subitement recourbé.

COURONNÉ. Une spire est conronnée lorsqu'elle est charáge au sommet de tubercules ou de pointes. Le cönce couronné impériale est couronné, il en est de mème de la tg-mbale conronne d'Ethiopie.

CRENEIÉ. Des bords crénclés, sont cenx qui sont dentés en créncaux et carrément ; celte particularité appartient notamment à quelques bivalves.

CRETE. Bords en crüte, formés en crête de corf : quelques huitres ont cetteforme, et il en est mème qui en portent le nom.

Créle. Des còtes peuvent ètre formées en clèie, surtont dans certaines coquilles bivalves, sourent elles sont armáes sur leur

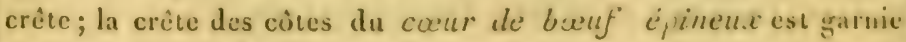
d'épines.

CROCIIET. Sommets de quelques genres de coquilles bivalves. Les sommets des moules se nomment crochets. 
Crochet. Dent faite en forme de petit croc. Les dents de la charnière des cœeurs, celles de la corbule forment le crochet.

CROCHU. Fait en façon de crochet.

CRUSTACÉ. Revêtu d'une croûte, d'une envcloppe solide, qui tient le milieu entre la corne et la matière calcaire. Les écrévisses, les crabes, les oursins sont crustacés. Il en est de même des bras de l'anatife.

CUNÉIFORME. Formé en coin, fait en coin.

CYLINDRE. Forme allongée, ronde, et ne dimiuuant rien de son diamètre; conservant la même dimension dans toute sa longaeur.

GYLINDRIQUE. Fait en cylindre.

CYLINDRACÉ. De forme cylindrique. Les maillots sont cilindracés.

\section{D.}

DECOLLÉ. Une spire est tronquée, décollée, lorsque ses premiers tours ont été alattus, et leur brèche réparée à plat par l'animal; quelques bulines ont leur spire décollée.

DECOUPÉ, qui n'est point uni : quelques ammonites ont leurs sutures découpées.

DECOUPURE. Fait en zig-zag, en feuilletis. Des bords déconpés sont l'opposé de bords unis.

DÉCURRENT : qu’ s'éloigne en s'avançant, en tounant; qui s'écarte du point de son départ: quelques calyptrés ont une lame décurrente; ee qui nous a engagé à en former un gene.

DENT. Plis saillans dont est chargée la columelle des mitres, volutes, turbinelles, et qui servent ì les caractériser.

Dent. Protubérances et crochets placés sur les. charnières des bivalves, et dont on fait le même usage.

Dent. Les bords faits en scie de ces mémes bivalves. sont encore dentés.

Dent. Màchoires dentées. Les limacons et. Zes limaces ont unc espèce de, màchoirc. 
Dent. On douncencore le nom de dent aux ossplets qqui sunt placés à la bouche des oursins, et quifont loffice de dents.

Dent. La pointe qui arme la bouche des licorne's vers sa base, porte anssile nou de dent.

DENTE: qui est garni de r'en's, qui porte des dents.

DEPRIMÉ, écrasć, aplati : yu dquess merex sout déprimṕs.

DERYAL. Les couleurs dermales sont celles çu'ou voit dans l'épiderme ou drap makin, et qui ne sont pas répétées sur le têt coquillier.

DESUXI. Séparé, non-joint. Les cilostomes ont leur bord arrondi et joint. Celui des toupies est désuni, il y a sulution do continuité.

DIAPHANE. Transparent, lucide, presque vitreux, mince et donnant passage au jour. Le carinaire, l'hale sont des coquilles diaphanes.

DIAPLIRA GME. Pièce intcrmédiaire, cloison placée au milieu de la cavité d'une coquille, et quelquefois même en reconvrement. Les crépidules ont un diaphragme qui forme leur cavité. ()rdinaircment les cloisoss sont multipliées, et le diaphragme solitaire.

DIGITÉ. Formé cn doights, fait en forme de doigta. I.a lève extérieure des ptérocires est garnie de pointes en forme de doigts, clle est digitée.

DIL ATF́, élargi, étendu. La lèvre extérieure des lambis, des ailes d'anges, ainsi que celle de plusicurs autres strombes, se dilate en aile. Cette lèvrest dilatée.

I)SIC.OIOE. En disque. Fait, formé en disque.

DISQUE. Forme elliptique et en disigue qu'affectent quelqques coqunilles. Le disque de l'haliotide est perforé, celui des s! omates et des sigarets u'a point de trons.

Disque. Les conrnes d'Ammon, les nautiles et les planorbes sont des coquilles formées en disque, en globe aplati.

Disque. Disque se dit encore du pied glutineux, on de la base musculeuse, an moyen desqucls rampent beaucoup de mollusques. C'est le pied des limace's et des limaçons.

DISSIV ILVE. Mollosique muni de plusieurs valres, mais non. 
réunies et dissidentes entre elles : de ces pièces testacées les unes le recouvrent, et les autres arment seulement quelques parties de son corps, sans être assemblées entre elles par des nerfs ou des charnic̀ros. Les tarets dont le corps est renfermé dans un tuyau, dont la tête est armée de denx valves, et dont le corps en porte deux autres, sunt des mollusques dissivalves.

L'organisation de ces mollusques, et celle de quelques autres qui leur sont congénères, nous a forcé à former cette nou velle division.

DIVERGENT. S'écartant l'un de l'autre en s'éloignant du point de départ. Les dents de la placune sont divergentes.

DORSAL. Du dos, appartenant an dos. Les carinaires ont une crête dorsale.

DOS. Partie extérieure et renflée d'un mollusque ou d'une coquille, et ordinairement la plus élevée. Le dos des nautiles est nni ; celui des limaces est réticulé.

DOUBLE. Des côtes, des stries peuvent être donbles on doublées. Ce sont celler qui se prolongent par paire, deux ̀̀ deux.

DRAP MARIN, ou épiderme. Substance sonvent cornue qui reconrre plusicurs coquilles; les cônes, les moules ont un drap marin.

DROIT. Sans courbure, s'élevant en droiture. La spire des tis est droite ; les baculites sont droits.

DROIT. Bord droit; c'est la lèvre extérieure d'une coquille dont la bouche s'ouvre a droite du spectateur, lorsqu'elle est placée sur sa base, et qu'il la voit en face: dans ce cas la columclle porte le nom de lèvre gauche.

Tonte coquille ainsi conformée est nne coquille à droite; et par conséqueut l'opposé d'une coquille à gauche.

\section{E.}

ECAILLE. Petite saillie unguiculée qui se trouve assez fréquemment sur le dos des coquilles bivalves; la partic postérieure du cour tricoté est écailleuse.

ECAILLEUX. Chargé d'écailles, portant des écailles. 
ECHANCRE. Qui n'est pas continu, mais découpé dans son prolongement. Les pleurolones ont la livre droite échancrée, et les houletles leur valve supéricure.

ECHANCRURE. Solution de continuite. Déconpure.

ECORCHÉ. Mis à nu, dépouillé. (Quelques molluryues perdent par le fiottement leur drap, on pran extérienre, et alors les couches intérieures on la nacre paroit à nu. Les somuets des anodontes et des maleltes sont écorchés dans le vieil àge.

ECLSSON. P'iece isolée, soit cornée on calcaire, ct renfermée sous la pean du dos. Les limuces ont un écusson; celui des sèclics porte le nom d'os.

Ecusson. On a encore attaché depuis pen cette dénomination it la partie centrale du corcelet, formée par le ligament dans les biralves; c'est le vulva de Linné.

EDENTÉ. Sans dents. Les pinnes oujamlnnneaux ont une sharnière élentée.

EGAL. Dans les bivalves, les valves sont égales lorsqua ì l’extéricur elles se ressemblent parfaitement en forme et en grandeur. Les cours, les moules ont leurs ralses égales.

Egal. Des stries, des cùtes sont égales, lorsque par leurs dimensions elles ne diflerent point les unes des autres.

Egal. Des plis égaux sont ceux qui ne difërent point nonplus entre cux.

Egal. Une sniface égale est une surface nnie.

FLLIPSE. Spirale ovale aplatic. $17 \mathrm{y}$ a yuel ques cognilles forinées en cllipse; tels sont les ellipsolithes.

ELLIPTIQUE. Formé en ellipse, approchant de l'ellipse.

EMBRASSANT. Qni reconve, qui embrasse: le manteau du mollusque des porcelaines cmbrasse toute la corquille.

EMOUSSÉ. Qui n'est pas pointu, dont la pointe cst émousséc. La spire des volutes est émoussée.

E.MPILÉ. Posé en nombre l'un sur l'antre, eı en ligne droile : les vertèbres des baculiles sont émpilées.

EMPILEMENT. Suite d'articles empilés les uns sur les antres. ENCIIASSt. Repris, serti, comme le sont les pierres pricienses dans l'or et l'arent, on sertissure des bijonr. 
ENCHASSEMENT. Reprise, sertissure.

ENFONCÉ. Qui n’est point releré, ni de nivean : la spire du planorbe, celle des ammonites sont enfoncées.

ENGAINANT. Qui renferme, qui enserre, qui contient, comme le fourrean renferine son épée.

ENGAINÉ. Recouvert d'une coquille engainante.

ENTAILLE. Découpure, brusque et profonde solution de continuité, Le pleurotome est entaillé,

ENTAILLÉ. Qui a une entaille.

En:aillé. Les bords des bivalves sont entaillés, lorsque les côtes, au lieu de se corrcspondre, alternent et se logent par leurs extrémités entre deux autres de la valve opposée.

Entaillé. Côtes entaillées.

ENTIER. Sans échancrure. Les scalaires, les ampulaires, les hélices ont leur bord entier et non-échancré.

EPAIS. Solide, fort. Les casques ont des coquilles épaisses ainsi que les strombes; les argonautes sont papiracés.

EPANOUI. Qui s'étend, qui s'élargit en éventail, en s'épanouissant du haut. Les pinnes sont des eoquilles épanouies.

ÉPIDERME. Enveloppe, surpeau membrancuse, cornée, qui reconvre la surface de quelques coquilles; les cônes ont un épiderme ou drap marin corné, les porcelaines, les olives n'ont point d'épiderme.

EPINE. Pointe allongée, droite ou recourbée. Les têtes de bécasse épineuses et quelques autres murex sont chargés d'épines.

EPINEUX, Qui a, qui porte des épines.

EPIPHR A GME. Cloison, fermeture dont les limaçons closent la bouche de leurs coquilles pendant l'hiver.

EQUILATERAL. Dont les côtés sont égaux; il se dit de quelques biralves dont les sommets se trourent précisément au milien des valves. Les péloncles sont équilatéraux.

EQUIVALVE. A ralves égales. Dont les devx ralves se ressemblent parfaitement, l'une offrant exactement la contre-partie do l'antrc. Lesmoules, les cours, les anodontes sont des coquilles équivalves.

E'TRANGLEMENT. Quelques coquilles sont successivement 
renflés et étranglécs : c’est ce quioa pent observer dans quelques ortocères.

EVASE. Très-ouvert; ouvcrute évastéc: les tgmbales, les tonnes ont une bonche évasce.

EVASE.MENT'. Ouverture tris-large, formce en vase.

EXCRE'TlON. Transudation, suintement, soit calcaire soit corné, au moyen desquels les mollusques testacés forment leurs coquilles et leurs opercules.

EXTENSIBLE. Susceptible de sétendre, de áallonger. Le col, les tentacules du liuagon sont tès-cxtensibles.

\section{F.}

FACETTE. Partie aplatic et unie du prolongement du sommet de la valve inlérienre dans quelques bivalves. Le sommet de la valve inférienre des spondiles a une de ces facettes ou aplatissement.

FENTE. Solution de continuité en longueur. Les siliguaires sont fendus dans tuute leur longueur.

FliNDU. Quia, quiporte une fente.

FESTON. Découpue en dents arrondies, et régulièrement placées à la suite les unes des autres; elles forment les bords festonnés.

IEUIILES. FEUIILETS. Lames minces couchées, appliquées et sourent toutes adhérentes sur leur plat les unes aux nutres. Solides, ce sont ces feuillets qui forment lépaisseur des coquilles que les mollusques tapissent graduellement par des couches nourelles, construction qui est la même dans les opercules soit calcaires, soit cornés, ain que dans lus écussums.

Feuilles. Feuillets. Les feuillets charuus constituent les branchies de quelques mollusques qui ont des organes respratoires fenilletés. Les branchies des huatres sont ferilletées ou faites en feuillets.

Feuilles. Fenillets. I.es cordons on hourrelets des chicoracés sont surmuntés et garuis defenilletsfrisés, et pressés les uns sur les antres.

FEUILLETli. Garni de fenillets, fait cu fenillets. 
FEUILLETIS. Suite de petits fonillets, se pressant les uns sur les antres; réunion de petits feuillets.

FEUILLURE, ou fenilletis; suite, série de petits fenillets.

FICHÉ. Planté debout, implanté dans la vase, le salske, ou entre les pierres.

FILET. Cils charnus, aigus, allongés, formés en fils. Quelques mollusques ont leur manteau bordé de filets.

Filet. Partie étranglée et sans renflement, qui attache un molInsque nnivalve à sa coquille : le nautile flambé est attaché de cette façon à la sienne par un filet qui traverse toutes ses cloisons.

FILIÈRE. Membre charnu qui sert à plusieurs bivalves pour porter les fils de leur bissus hors de leur coquille, afin de s'attacher anx corps solides : les mollusques qui habitent les moules, les perles, les marteaux sont pourvus d'une filière.

FISSURE. Fente mince, petite solntion de continnité. Les émarginules ont une fissnre sur le bord de leur coquille.

Fissure. On donne encore improprement ce nom à un tron qui perce le sommet des fissurelles.

FIXÉ, attaché. Une coquille fixée est cclle qui est attachée à un corps quelconque. Les huîtres se fixent anx rochers par le dos dc leur coqnille, les Lalanes par le pied, les anatifes, les anomies par un pédicule, et les moules par un bissus. Tons ces mollusques sont fixés.

FLUVIATILE, desfleures, appartenant aux eaux furiatiles : sous la dénomination de filuriatiles on comprend toutes les coquilles des fleuves, des ruisseaux, des étangs, des marais, des fossés, toutes celles enfin qu'on rencontre dans les eaux douces. Les planorbes, les anodontes sont des coquilles thuviatiles.

FONTINAL, des fontaines, appartenanb aux fontaines. Il y a des bulimes fontinaux.

FORE. Percé d'un on de plusicurs trous. Le disque testacé de l'haliotide est foré à jour: l'axe de la piramidelle ue l'est que jusqu'au sommet.

FOSSETTE; cavité qui renferme un ligament. Les fossettes sont placées à la charnière de quel ques bivalves. 
Fosiche. Les dents des charniores sont encore rerues dans les fossetles quileur sont opposées.

FOURCHU. Fait en fourche, à deux dents.

FRAGILE, cassant, se rompant, se lrisant facilement. Les spirules, les carinaires sont des conquilles frawiles.

FRAISE. Téte percée de trous de l'arrosnir : sa pomme.

FRANGE. Pordé de cils ou de filets nombreux, et disposéx en frange: quelques mollnsqunes ont un mantean frangé.

FLSEAU. Cylindre allongé, et insensiblement aminci par les denx bonts.

ILSIFORME. Fait en forme de fusean. Les rostellaires sont des coquilles fuisiformes.

\section{G.}

GATÉROPODES. Pieds sons le rentre. Dénomination qui s'appliqune a un très-grand nombre de mollurįues rampans. Les limaces, les limaçons et un grand nombre diunivalses sont gastéropodes.

GAUCIIE. La gauche du spectateur; une copuille à ganche est celle dont la bouche est inserse à celles tournées ì droite; les copuilles à gauche sont assez rares; il en existe cependant des genres tout entiers : les turrilites sout des coquilles à gauclie.

Gonche. La levre gauche dans les uniralies est celle à grauche du spectateur; dans les coquilles à droite, cest la columelle.

GiLATINELX, transparent, tremb]ant, en gelée. Il y a des mollusques gélatineux.

GIBBELX. Bossu. La grimace est une coquille gibbense.

GLABRE. Lisse, uni, poil. L'ivoire est une coquille glabre.

GLOBULEt F. Fia en globe, arrondi en boule de la même manière que les bulles.

GRAIV]. Clargé de grains, de petits tubercules ronds et preasés; la pomme de Grenade est chasuce de grains.

GRE VETIS. Assemblage de petits grains; la pomme de Grenade, le cóne amiral de Caragao ont un grenetis. 
GOUTTIÈRE. Creux, renfoncement prolongé et superficiei; les belemnites ont une gouttière.

Gouttière. Un canal fait en bec et très-alongé porte encore le nom de gouttière; les tétes de bécasse ont le bec fait en gonutière.

GROUPE. Réunion, assemblage en famille et adhérent. Les luîtres, les anatifes forment des groupes.

GROUPÉ. Réuni, assemblé, disposé en gronpe.

\section{H.}

HERBIVORE. Mangeur d'herbe, qui se nourrit arec des herbes; beaucoup de mollusques terrestres, les limaces, les limaçons sont herbirores.

HÉRISSE. Cihargé, rempli de poils, d'épines; les spondiles sont hérissés de pointes; les cames, par des ćcailles.

HERMAPHRODITE. Mâle et femelle tout ensemble, qui réunit les deux sexes. Une immense quantité de mollusqaes est hermaphrodite.

IIISPIDE. Une surface est hispide lorsqu'elle est hérissée de poils.

\section{I.}

IMBRIQUÉ. Disposé en tuiles, en recouvrement : l'oscabrion est reconvert par des pièces imbriquées.

IMPERFORÉ. Qui n'a pas de trous, qui n'est pas percé : les patelles, les stomates sout imperforées.

IMPRESSION. Trace, marque; les muscles qui ferment intírieurement les bivalves, laissent des impressions qu'on nomme musculaires: les huîtres n'ont qu'une impression musculaire, les vénus en ont deux, et les anodontes en présentent trois dans l'intéricur de leurs valves.

Impression. Le nerf, lien ou ligament corné qui réunit denx valves à leurs sommets, laisse aussi son impression, clle se nomme l'impression du ligament, et elle est très-remarquable sur la facette des spondiles, ainsi que sur le sommet des huîtres. 
INA URIC:ULE. Sans oreilles, dépourvu d'oreilles: les icllines sont inaurioulécs.

INCiLIN É, Recourbé sur lui-mème, ou rejeté en arrière sur ha coquille.

Incliné. Dévier de la ligne droite; des cùtes peuvent être inclinces à droite ou à gauche.

\section{INEGAL. Raboteux, peu uni.}

Ineigal. Les valves inégales, sont celles qui sont plus grandes ou plus petites dans le mème individu: les balanes ont une grande valve et quatre petites.

Inegal. Des ralves des corbules sont encore inégrales, parce qu'il y en a une qui est constamment plus grande que l'autre.

IM LUUIL.A'TERAL. A còtés inéganx : les valves inciquilatérales sont celles dont les sommets sont plus éloignés d'un lonu de la valve que de l'autre. Les valyes des tellines sont inéquilatérales.

IVÉ(jUIVALVE. A ralves inégales, ayant des values iniggales, et dont l'nie est plus grande ou plus bombée, on autrement formée que l'autre; les peignes, les corbules, les huïres, les spandiles, sont inéquiralves.

INFERIEUR, Valve inférienre, valve de dessons; clle est orilinairement plus bombée que la supérieure, et très-sourent aussi plus pâle: les peigne's, les lacuitres, les pandores ont une valve inférieure.

INEUKDIBULIFORME. Fait en forme d'entonnoir, formé $\mathrm{cn}$ entomuir. Quelques calyperces sont faits en forme dientonnoir.

INSECTE, INTEMSEC'TÉ. Liranglé par des espeeces de conpures; par des intersections comme des insectes. (Quedynes coguilles sunt intersectées.

INTERNE. En dectans, qui est intérieur : les impressions musculaives sont internes dans les bivalves.

INTERSTICE. Séparation. l.es dents sériales des pernes présentent entre elles autant d'interstices.

Interstice. Distance qui exinte sur le dos des coynilles entre un bourrelet ou cordon. et cclui qui précede ou qui suit ; les hourrelets des scalaires présentent desinterstices. 
Interstice. Les côtes des cœurs laissent entre eux des interstices.

INTRANTE. Lorsque les charnières des bivalves ont des dents qui s'engrainent, ces dents sont intrantes.

INVERSE. En sens opposé; les deux valres des biralres se dessinent en façon inverse.

INVOLUTE. Sans rolute, sans spire, non-spirale : les patelles, les fissurelles sont des coquilles in rolutes.

IRISÉ. Reflétant, nacré, poli, luisant, offrant toutes les conleurs de l'iris.

IRREGULIER. Qui n'est point sur un même plan, sur une même ligne, mais ondulé; les bords des bonnets de dragons sont irréguliers.

Irrégulier. Lorsque les hords d'une coquille ne sont point constamment les mêmes, ne dessinent point toujours les mêmes coutours, ils sont irréguliers; les crépidules, les huîttres ont leurs bords irréguliers.

ISOLÉ. Senl, qui n'est point rénni, qui n'est point groupé ; les anomies sont is olćs sur leurs pédoncules comme les lingules. Les harpes, les vis, les mitres vivent isolés.

ISOLEMENT. Vivre dans l'isolement, rester isolé.

ISOLEMENT. Vivre seul, virre isolément, sans former de réunion.

JUXTAPOSITION. Adjonction perpétnelle et successive de mollécules calcaires ou cornées, transsudantes du mantcau, du corps et du pied des mollusques testacés ou coriacés : ce sont ces mollécules qui forment par des couches sourent répétées les enveloppes, les têts ou coquilles, les opercules, les écussons, les os de ces mollusques.

\section{L.}

LAME. Espèce de diaphragme, pièce formant séparation : certains calrptrées ont une lame décurrente. 
LA.MFI.LIF()KML. En forme de lame, fait cn lame.

1.AVGUi'T'TE. P'ièce tectacée intéricure, adhérente à la coquille, et formée en langue; quelques cabochons ont une languette, on la retrouve encore dans les niyes.

LATÉRAL. De côté, appartenant au côté.

L.ATLRALEMLN'T. Placé latéralement, placé à côté.

LEVTICLAME, Fait en lestille, en forme de lentille; les nummulites eu les radiolibhes sunt des coquilles lenticulaires.

Lenticulaire. De la grandeur d'une lentille; des lamilles cutières de coyuilles sont lenticulaires.

Liv RE. Dernier tour de spire dans les univalves; son bord on partie extérieme, arrêté quelquefois par un cordon ou bourrelet.

Livre. Les porcelaines, les orules ont deux lèrres, l'une gauche et l'autre droite.

Livre. La columelle porte anssi quel(quefois le nom de lèrre.

Lère. Dans les bivalves, les lères sunt le bord extérienr des valves.

LIGliE. Point attaché, mais fottant, nageant ou roguant librement; les cúnes, les zolutes sout des mollusques libres.

LIGAMENT. Nerf, attache cornéc faisant ressort qui réunit, comme dans les huîtres, les deux valves par leurs sommets. Presque cons les mollusques bivalves ont leurs valves reunies par un nerf ou ligament. Celui des huâtres est intérieur, on le roit à l'extérieur dans les moules.

L.MBE. Circonférence intérieure des valies contre leur rebord.

LINÉAIRE. En ligne, de l'épaisseur d'une lignue.

L.INGUIlOR.ME. In maniere de langone, furmé en langue.

LISsE. Uni, sans plis, sans tubercules; les marginelles sont des coquilles lisses.

LITHE. Terminaison des noms de pétrifications, du greec lithos, pierre. Des mum:dithe's, des na:tilithe's, sunt des mumules, des nautiles pétrifiés.

I.OBE. Dácoupure faite en forme de larģe feston, mais irréguliere et allongée par une espice d’étranglement à ad base ; quelques 
cornes d'Ammon, des pélagulithes ont les découpures de leurs cloisons formées en lobes.

LOBÉ. Formé, dessiné en lobe.

LOGE. Espace compris entre deux cloisons dans les coquilles chambrées ou polythalames; les nautiles, les ammonies, les orthocères ont desloges.

LONGITUDINAL. Eu longueur, s'étendant en long; les arches ont une suite longitudinale de dents; leur channière est longitudinale.

LOZANGE. Carré long et posé sur sa pointe, maillé en lozange.

LOZANGE. Marqué de lozanges, reconvert par des lozanges.

LUNAIRE. Fait en croissant, en demi-lune; ouverture lnnaire.

LUNULE. Endroit plus ou moins remarquable placé sous les crochets et postérieurement dans quelques genres de bivalves; les tridacnes et les hippopes ont la lunule ouverte, ou baillante, elle est close et fermée dans d'autres espùces.

\section{II.}

MACULÉ. Taché, coloré, couvert par des taches larges et fondues.

MAILLE. Dessin formé carrément, semblable aux mailles d'un filet.

MAILLÉ. Couvert de mailles, dessiné, formé en réscau.

MAMELON. Pointe, protubérance arrondie et obtuse. Les tymbales, les volules ont leurs sommets formés en mamelons.

MAMELONNÉ. Fait en mamelon, clargé de mamelons.

MANTEAU. Prolongement charnu, membraneux, musculeus faisant partie du corps des mollusques, recourrant quelqueiois leur coquille tout entic̀re, mais s'arrêtant ordinairement à la bouche des coquilles univalves, la rebordant intérieurement, et formant par la transsudation de ses hords la robe extérienre de ces coquilles; dans les bivalves, ce sont encore les bords du nanteau 
qui ajontent perpétucllement à ceux de la coquille, en formant ct colorant leurs valres.

MARÉCAGEUX. De narécage, les coquilles marécagenses sont celles qui vivent et se multiplient dans les marais, et autres endroits bourbeux.

M.IRGE. Partic extérienre, dos des nantiles, desconnes d'Ammou, et de toutes les coquilles contunuées en spirale.

MARGINAL. De la marge, du côté, à la marge ; un tule marginal est celui situé contre le dos, ou la partie la plus extrieure et la plus arrondie, Je quelques cornes d'Ammon; an lien d'ére au centre des cloisons ou contre la spire; le tube de plusieurs pélagolithes est marginal.

MARINE. De la mer : on distingue les coquilles en roquilles marines, terrestres et lluviatiles. Les cônes, les zolute:, les huîtres sont des coquilles marines.

ME.MBRANE. Tissu, peau, pellicule d'une certaine tenacité, et ne servant pas i recourrir médiatement la chair ; susceptible mème de s'ealler en forme de vessie. Les janthines ont une memJrane qu'ils enfent en forme de grappe de raisin, ou de bulles de savon.

MEMBR.LNECI. Tissu membrancux, formé en manière de membrane.

\section{Membraneux. Revêtu d'une membrane.}

MICROSCOProue. Peu ou point visilıle à l'veil nu, de la grandeur d'un grain de sable; les sables des bords des mers sont remplis de coquilles microscopiques. Soldani a fait un tres-bel ouvrage sur les coquilles microscopiques.

MOLLLisgUE. Animal à corps mou, sans vertèbes ni charpenteossruse; on général le sang des mollnsynes est blanc, ou plutôt c'est une espoce de lymphe. Ces étres forment une classe tres-nombreuse dans te rérne animal : il y en a do nus, de crustacés, de testacés, de coriacés et de gélatineux.

MURTE. Une coquille est morte lorsquabandomóce à la mort de son mollusque, elle a été roulée par les flots an point de pacrle ses couleurs.

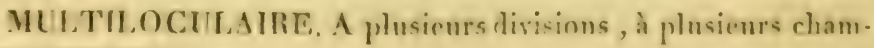


bres, coquilles chambrées et polythalames : ces coquilles sont surtont très-nombreuses parmi celles pétrifiées et nicroscopiques. Le3 hippurites, les nautiles, les chélies, les spirules sont multiloculaires.

MULTIVALVES. Ayant plus de deux valves, mais réunies entre elles par des nerfs ou ligamens, et formant charnière. L'anatife, le pholade sont des coquilles multivalves.

MUSCLE. Organe, masse charnue, flexible, contractile, fibreuse, au moycu desquels les mollusques, et surtont cenx bivalves, sont attachés à leurs coquilles; les muscles sont intérieurs, et servent à l'animal pour tenir leurs valves ferıcées: les huittres ont un seul muscle ainsi que les moules, les cœurs en ont deux, les anodontes comme les mulettes referment leurs valves avec trois.

MUSCULAIRE. Semblable à un muscle, de substance musculaire.

MUSCULEUX. Fait en muscle, formé de muscles; le pied sur lequel rampent les limaçons, est museuleux.

\section{N.}

NACRE. Irisé, reflétant tontes les couleurs de l'iris, de l'arcen-ciel. Les nautiles, plusieurs toupies, le cantharide, les mulettes sont nacrés intérieurement.

NAISSANCE. Origine, base; la naissance des tentacules est l'endroit où elles sont rénnies à la tête, au corps de l'animal; celle des filets du manteau est celui de leur point de départ. Les mollusques habitans des natices ont les yeux à la base ou naissance extérieure des tentacules.

NAVICULAIRE. De forme naviculaire, fait en manière de petit vaisseau; les nautiles, les carinaires sont des coquilles naviculaires.

NÉOLOGIE. Termes, expressions nouvelles et étrangères à la langue en usage et parlée; mais admises et consacrées dans une science quelconque. 
YERF. Jignament corné qui réunit les denx valves des coquilles bivalves, et qui parson ressort les fait ouvrir.

NODUI.fil. A . Ayan des nocuds, chargé de tubérosités en forme de uouds, ayant des renflemens; les bras de quelques velelles sont uodulenx.

NOEUD. Renflement, glande.

NON-ADHERE.VT. Sans adhérence, saus réunion; le limarnn ferme en hiver sa copuille ares une espece d'opercule non-adherent à l'animal, les ralves de l'acarde n'adhirent point l'une i l'autre par un ligament.

NOUELX. Chargé de monds, derenflemens; les pointes qui arment la livre droite du plèrocerescorpion noueux sunt chargées de noends.

NC. Mollusque dont le corps cst nu, sans tit, sans coquille ou enveloppe solide quelconque; les siches, les poulpes, les calmars sont des mollusques nus.

NYMPLES. Impressions, restes et indiention de l'emplaer-

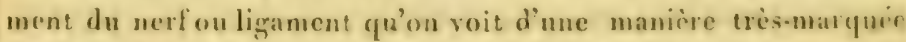
dans quo'ques bivalves, et notamment dans certaiues véuna. Cies impressions doivent alors ètre placies sous les crochets ou snmmets, en avantet au centre du corcelet. I.es uymphes de la z'énus dionéc, ou congue de frénus, sont iresapplarentes.

\section{O.}

OBI.IกUT. De côté, dirergent ì ganclse on à droitc; s'éloi. guant des lipnes horizontales et perpendieulaires; quelques bivalses ont des stries ubliques. Les plis de la columelle des mitros sont obliques.

OBLIOLEMEXT. Rui est place, formé, disposé d'une manière oblique.

Obi.ONG. Conuille oblongue, arrondie ct allongée. Corpsun peu plus long que large.

OBTUS. A pointe écrasée en mamelon; la spire des volubes est obluse à son sommet. 
OESOPHAGE. Orifice supérieur de l'estomac, on sac alimentaire chez les mollusques.

OEUF. Les mollusques ont des oeufs, et par conséquent ils sont ovipares; l'hclix vivipare lui-même l'est, ses neufs sont carrés et renfermés en août sous son mantean dans sa coquille.

OGIVE. Fait en ogive, en fenêtre gothique, en voùte aiguë; quelques coquilles cloisonnées ont la bonche faite en ogive.

OMBILIC. Creux, trou, ouverture centrale formée dans l'épaissenr et an centre de la columelle, et souvent même par les tours de spire; les cadrans sont ombiliqués, et d'antres coquilles encore sont munies d'ombilics.

OMBILIQUÉ. Qui a un ombilic, qui est formé en ombilic.

ONDÉ. Fait en ondes, se relevant et s'abaissant buccessivement comme l'eau agitée.

ONDULÉ. Formé en petites ondes, en ondulations.

OPERCULE. Pièce ordinairement arrondie, solide, cornée on calcaire, qui recouvre ct ferme dans l'état de repos la bouche des coquilles dans beatucons d'univalves. Ordinairement ces opercules sont adhérens au pied de l'animal. Les veuves ont un opercule corré, celui des burgaux est calcaire. On a improprement donné aux opercules calcaires lenticnlaires le nom de pierres d'hirondelle.

OPERCULÉ. Qui a un opercule, bouche ferméc par un opercule, pied operculé. Le mollnsque des turritelles a le pied operculé.

OPPOSÉ. En regard, vis-à-vis l'un de l'antre. Les valves de l'acarde, qui ne sont point réunies par un ligament, mais retenues senlement par un muscle, sont opposées.

ORBE. Rond aplati, mais cependant un peu renflé, coquille eli orbe, coquille arrondie.

ORBICULAIRE. Fait en orbe, formé en rond.

OREILLE. Les peignes en général ont des oreilles.

ORIFICE. Le manteau de quelques mollusques est quelquefois fermé, à l'exception d'un ou de deux trous on orifices; e'est ainsi encore que les tarets ont denx orifices à la partie postérieure de leurs corps. 
OS. C'est ainsi que l'on nomme un large et long écusson calcaire que la seche porte dans le dos entre cuir et chair.

OSSILET. Piece calcaire intérieure adhérente à l'estomac de quelyues mollusques, et qui sourent est accompagnée de deus antres. Les bulles ont ainsi trois pièces calcaires qui constituent leur estomae; et c'est de ces trois prièces réunies qu'on aroit rouln constituer un genre sous le nom de gioenie.

OSSEUX. De nature d'os, de substance ossense.

OUVERTURE. Bouche des coquilles univalves; les sabors ont la bouche ronde, les volute's, les casques l'ont échancrée.

OVAIRE. Organe renfermant les oufs avant quils soient cxpulsés du corps de l'amimal, il constitue le sexe féminin.

Oraire. Membrane libre, souvent digitéc et formce cn fleurs, renfermant lé embrions des mollusques testacés; l'ovaire des buccins ressemble par sa forme générale à des clous de girnfle qu'on auroit implantés sur une membrane les uns à côté des autres.

Oraire. L'ovaire que jettent anuuellement les seches et les calnars, est formé en grappes; celui du calmar en présente une multitude rénnies à un point commun et central.

OVAL. En forme d'oeuf, fait en forme d'oenf.

OVIPARE. Qui donne, qui jette des oufs; qui se multiplie, qui se propage par des oeufs ; les mollusques sont ovipares: le limagon jetie ses oufs par une ouverture qui se trouve à cúté de la tète, à l'endroit où d'autres animaux ont l'orcille.

\section{P.}

PANACIIE. En forme de plumes d'autruche, ramifiration épanouie. Les branchies de quelques mollusques présentent des panaches.

PAPIRACÉ. Léger, mince comme une feuille de papier. Les argonaules sont dés coquilles papiracées.

PARALLELE. Còte à cóte, conservant une égale distance; deux lignes sont parallibles lorsque sans dévier elles conservent 
cntre elìes constamment le même éloignement; des côtes, des cordons sont quelquefois parallèles.

PAROIS. Surface inférieure et supérienre des cloisons qui forment les chambres ou séparations des coquilles polythalames. Les parois des cloisons des nautiles sont nacrés, cenx des hippurites sont ternes, et de couleur jannâtre.

PÉDICULE. Pied qui remonte, qui s'élève en devenant plus mince qu'il ne l'est à sa base; les pleurotomes sont montés et rampent sur un pédicule qui s'élargit par le bas en manière de pied.

PÉDICULÉ et PÉDICELÉ. Qui est monté, placé sur un pédiculc.

PÉLAGIEN. Qui habite, qui reste au fond des mers; on regarde les anmonies comme des coquilles pélagiennes.

PÉLAGIQUE. Dn fond des mers, des abîmes profonds do l'océan.

PERFORÉ. Percé. L'axe de la piramidelle est perforé jusqu'au sommet dela spire,

Perforè. Le disque des auricules, et le commet des fissurelles est perforé.

PERSISTANT. Qui ne s'efface, qui ne s'enlève point ; constant et se formant à chaque période: les bourrelets de beaucoup de murex sont persistans, les côtez des casques sont persistantes.

PIED. Disque ou pied, faiscean de muscles charnus sur 1equel un mollusque rampe, et qui lui sert de pied. Le limaçon rampe sur son pied, il en est de mêrme des lépas.

Pied. Base ou pied d'une coquille lorsqu'elle est posée ou attachée sur sa base. Les balanes sont fixés par le pied de leurs coquilles.

Pied. Tube, ou pédoncule sur lequel sont portées d'autres coquilles. Les anatifes ou pousse-pieds, et les térébratules sont portés sur un pied.

PILEUX. Chargé de poils courts et de nature cornée, il y a une arche pileuse; le drap marin de certaines coquilles est pilenx. 
P'IIfIORME. En forme de poils, fait en façon de poils.

PISHORME. Resscmblant à des pois, formé en pois.

PLANE. Uni, plat; une surface plane est platte et polic: les placunes sont des coquilles planes.

PLiS. Dents dont sont chargées Ies columelles de beaucoup de mollnsques univalres, et qui servent ì caractériser leurs genres; les volutes, les milres ont des plis, les harpes en sont dépourvus.

Plis. Lorayue les lères des bivalves dévient de la ligne droite, lenrs hords forment quelquefois un pli ; c'est ainsi que les tellines ont un pli qui les caractérise.

PLISSÉ. Chargé de plis, formé par des plis. On dit qu'une coquille unisalve est plissée, lorsque ses còtes sont très-pressées et qu'clles semblen former des plis.

POLYTIALAME. Chambré, cloisonné, ayant plusieurs concamérations : les nautiles, les orlhocères sont des coquilles polythalames.

PORES. Petits trous très-multipliés, très-rapproclıés les uns des antres. La tête des arrosoirs est poreuse.

POREUX. Rempli de pores, ou de petits, trous très-rapprochés.

POSTÉRIEUR. En arrière; bord postéricur, cûté postéricur.

PROÉMINENT. Saillant, élevé par-dessus; une spire prociminente est le contraire d'une spire enfuncée ou écrasée.

PRO'TUBÉRANCES. Elévations roisines les unes des autres et multipliées. La mure est recourerte de protubérances.

PROTLBERANT. Saillant ; quelques coqquilles ont sur leurs cordons des épines protubérantes : les dents dont les charnières des biralres sont armées, sont protubérantes.

PYRLOORMF. Fait en forme, en farcon de poire: les firues, les provules sont faites en poires, clles sont pyriformes.

\section{Q.}

QQLADRAYGUTAIRF. Carré, une nuverture quadrangulaire 
est une bouche carrée; les toupies ont la bouche presque quadrangulaire.

QUADRANGULAIREMENT. Coupé carrément, à angles droits ; les stries et les còtes du cancellaire, pas de parsan, sont conpés quadrangulairement.

QUADRIVALVE. Arrué de quatre valves; indépendamment de leur valve principale, les balanes ont la bouche armée de quatre autres petites valves.

\section{R.}

RACCOURCI. Devenn plus court. Les tentacules des limaçons rentrent en eux-mêmes, leur pied se contracte, et lenr corps se raccourcit.

R.ADlE. En rayons, rayonnant. Les stries colorées de la telline, soleil levant, sont radiées, elles rayonnent.

RAMEAU. En ramean, qui s'épanouit, qui se divise; les branchies de quelques mollnsques sont formées en rameanx.

RAMEUX. Fait en rameaux, en s'épanouissant, en se divisant en branches.

RAMIFIÉ. Se divisant en branches, fait, formé en rameaux.

RAMPANT. Mollusques rampans. Les mollusques ne rampent point tousà la manière des serpens, ils semblent se traîncr, se glisser; ils avancent et marchent en imprimant un mouvement d'ondulation à leur pied.

RAMPER. Marcher, se traîner à la manière des limaces et des limaçons.

RAIE. Strie fine, ordinairement dans le sens de l'accroissement de la coquille; les raies du cône écorché sont très-fines.

REBORD. Bordure, bord, le rebord du mantean, le rebord des lèvres des coquilles; la lèvre extérieure des marginelles a le rebord renflé et formé en façon de bourrelet.

liECOURBE. Plié en coude, courbé, replié; les cérites ont un canal recourbé.

RECOUVRANT. Qui conrre, qui recourre entièrement l'ani- 
mal. Jes parciles, le's fissurelle's, ley concholejas sunt des coquilles recourrantes.

REFHECIII. Des borls réfléchis, surplombés, surhordés, qui débordent leur pied, leur base; les burds ou cordons des sealaires sont rélléchis.

RE.VHLF. Devenu plusépais, plus ggros ; beancoup de cascjues ont le bord extérienr renllé.

RENGLEMENT. Espèce de bourrelet; ce mot s'applique principalement anx leves des miralves qui s'épaississent dans leur bord : le hord extrienr des marginclles est renflé.

RETICULE. Fait en réseau, en filet, couvert de mailles.

IEETOUR. Retour de la spire. La bourhe des nauliles, des cornes d' Immon reçoit le retour de la spire. Dans les spirnles il conserve son indépendance.

RETRACTILE. Susceptible de se retirer, de rentrer en luimème, en dedans, dans l'intérieur; les tentacules des linaçons, la trompe des tarets sont rétractiles.

RE'TRECI. Un tuyan se rétréd lorsyu'il diminue de diamètre. La tigge des arrosoirs se rétrécit par le bas.

REUNI. Qui n'est plus divisé, qui n'est pas séparé, mais conjoint; le hord de la bouche des cic.ostomes forme le cercle, ce bord n'est plus divisé, mais il est réuni.

REUNION. Accouplement; beancoup de mollusques ne s'acconplent que dans des réunions ordinairement printanieres; les planorbes s'accouplent dans de grandes réunions, ì cette difuque ils forment de longs chapelets.

RIMULE. Petite fente, légère scission, quelqques coquilles cloisounées dont la bouche est recourerte, y ont une rimule.

RIVURAL. Provenant des rives, habitant des rives. Les mollusques qui habitent lesplages, les rives de la mer et des rivieres sont des mollusques riverains, et leurs coquilles se nomment rivurales.

ROBE. Parlie extririeure, surface d'une coquille; la robe de beancoup de porcelaines est coloríe, celle desovules est blanche.

ROMPU. Sans continuté, en sursant; des rayes, des stries en sursant sont des raies et des stries rompues. 
ROSÉ. De couleur de rose, teinté de rose. Quelques strombes ont la columelle teintée de rose.

ROSTRE. Bec formé en éperon et fort allongé; les têtes de bécasse épineuse, les massues d'Hercule ont au lieu de canal à leur base un rostre fort allongé.

ROSTRÉ. Qui a un rostre, un canal eu bec fort allongé; les coquilles que nous venons de citer sont rostrées.

ROULÉ. Une spire estroulée; les tours de spire se contournent, se roulent en recouvrement les uns sur les autres.

Roulé. Une coquille roulée est encore une coquille abandonnée par son animal, une coquille morte, ronlée par les flots, et dont les angles, les pointes et les tubercules sout usés par le frottement.

RUGOSITÉ. Sillon, creux prolongé ; une côte est placée entre deux rugosités.

RUGUEUX. Sillonné, traversé par des stries, qui n'est pas uni, mais âpre an touclier; fait en lime, en rape : les limes ont leurs valves rugueuses.

S.

SAILLANT. Elevé, hors-d'œuvre, protubérant; les feuillures des chicoracées, les côtes de la conque exotique sont saillantes.

SAILLIE. Elévation, reuflement. L'avant-dernier tour de la spire des nautiles forme une saillie dans leur bonche.

SCABRE. Ecailleux, âpre; les cames sont scabres extérieurement.

SCELLÉ. Fermé momentanément, mais hermétiquemeut ; la bouche des limaçons ( helix) est sceliée en hiver par un épiphragme et quelquefois même, s'il est rigoureux, par derıx ou trois.

SÉCURIFORME. En forme de hache, fait en manière de hache. Le pied musculeux des peignes est sécuriforme, il est fait en forme de hache.

SEMI. Presque, demi, approchant; une coquille scmi-ronde est demi-ronde. 
SEMI-LUNAIRE. Formé, fait en demi-lune; il $y$ a des opercules sémi-lunaires.

SlililAL. Suite régulière, en série et régulière; les dents des arches, des pernes sont sériales.

SKRlile. Suite, continnité, objets placés les uns à cûlé des antres; une série de dents, de puintes, de côtes.

SEliRATE. Formé en dents de scic; les plicatules ont les bords de leurs valves serratés.

SESSILE. Des yeux sor.t sessiles lorsquils ne sont point montés sur un pédicule.

SILLON. Linne creusée et prolongée, placée entre deux élévations ou crêtes.

SILLONNÉ. Crensé, traversé par des sillons, par des raies profondes; le conca veneris, ou venus dionce, est sillonné.

SIMPLE. Se dit d'une coquille qui n'est pas composée, qui n’a nispire, ni languette, ui cloison intérieure; les patelles sont des coquilles simples.

SINUEUX. Ondé, replié, profondément lo'sé, déconpé en rondeur; certaines cornes d'Ammon, ainsi que des nauiles, ont des cloisons dont les bords sont sinueux.

Sinueux. Le bord ou lères des coquilles univalses sont sinueur, lorsque leur intégrité est interrompue par un sinus; le bord des pleurotomes est sinueux.

SINUOSITE. Cioupure, découpure ondulée; les sinuosités d'une cloison.

SINUS. Echancrure, découpure profonde, qui paroit séparer ou conper en deux la lève extérieure des pleurotomes; les clavatules ont aussi une espèce de sinus.

SIPIION. Trou ou tube prolongé, et se continuant au travers des cloisons des coyuilles chambrées. Les naulile's, les ammonices ont un siphon.

SOM.ME'T. Parlie la plus élevée; dans les coquilles uniralves c'est la pointe de la spire.

Sommet. Cher. Le's bivalyes on nomme sommets des ralves lit parlie suprérienre et ordinairement recomiée en come dabon. dance, opposíe aux hords, et qui surmonte en dehors la clars- 
nière ; c'est sous les sommets que le ligament ou nerf tient les denx valves attachées l'une à l'autre; les sommets des anodontes sout écorchés, ceux de l'isocarde, ou bonnet die fou, contournés.

SPATULE. Fait en bec aplati et arrondi, en spatule; les tarets ont deux petites valves spatulées.

SPINCTER. Ouverture resserrée et plissée sur scs bords orm dinairement faite en cul de poule.

SPIRALE. Fait en forme de spire; les ammonies, les pélagolithes sont des coquilles spirées, faites en spirale.

SPIRE. Ensemble ou totalité des contour's en spirale formés par les mollusques uniralres, et constituant leurs roquilles; la spire des mitres, des vis est élevée, celle des volutes plus courte et celle des planorbes aplatie.

SPIRE. Muni d'une spire saillante ou écrasée.

SPIRIVALVE. Les mollusques spirivalves sont cenx qui portent une coquille formée en spire; les strombes, leв buccius, les olives, sont des coquilles spirivalves.

SPONGIEUX. Rempli de pores, de trous, ayant plus de vide que de plein; ressemblant à une éponge, fait en forme d'éponge.

STAGNAL. Des étangs, provenant des étangs, habitant les étangs ; leurs eaux nourrissent des coquilles qui leur sont particulic̀res.

STIGMATE. Cicatrice, marque; une coquille qui a étéblessée pendant la vie de l'animal, porte des stignates de ses auciennes blessures.

STRIE. Raie très-fine; les stries sont des raies fines et nombreuses, quelquefois en travers, d'autrefois en long ; ordinairement les striës marquent l'àccroissement journalier et progressif des cocpuilles; les stries dn cóne drap d'or sont très-pisibles quöique très-fines.

STRIÉ. Chargé de stries, de raies très-fines et multipliées; indépendamment de ses côtes le dos des harpes uffre des stries.

SUBAURIFORME. Presque en forme d'oreille: lorsque la 
syllabe sub est préposće à quelque mot, c’est un diminutif, et il signifie presque dans le langage conchyliohogique.

SUBCiANALICULE. Presque en canal.

SUBCORDIFORME. Presque en forme de coeur.

SUBCILINDRIQUE. Presque en forme de cylindie.

SUBDISCOIDE. Presque en disque.

SUBÉQUILATÉRAT. Presque é(quilatéral.

SUBÉ:OUIVALVE. Presque équivalre.

SUBIUSIFORME. Presque en forme de fuseau.

SUBGI.OBULEUX. Presque en globe.

SUBORBICULAIRE. Presque orbiculaire.

SUBPISIFORME. Presque en forme de pois.

SUBPYRIFOR.ME. Presque en forme de poire.

SLBTRIGONE. Presque triangulaire.

SUBTURRICULÉ. Presque en tour.

SUTURE. Joint denté, formé en scie, persillé ; quelques ammonites ont des cloisons avec des sutures persillées. Quelquefois les sutures sont simplement lobées.

Suture. Fente formée en dents de scic.

Suture. fiéunion de deux parties, qui affecte les mênes dents.

Suture. Ligne apparente sur la spire des univalves, et qui est formée par la révolution de ses tours. La spice des fuseaux, des vis, a des sutures très-apparentes.

$$
\mathrm{T} \text {. }
$$

TEINTÉ. Légèrement et largement coloré; la columelle des sirombes lambis est teintée en beau couleur de rose.

TEVDINEUX. Muscles rigides et tris-fermes, de la nature des tendons, tendineux, presq̨ue cartilagineax.

TENTACUIE. Ce sont des filets creux, fermes ou charnus, susceptibles de contraction, sans articulations, doućs d'nne très-grande sensibilité ; les corr̈es dont la tête des lima- 
çons est gुarnie, sont des tentacules; ils paroissent servir à ces mollusques pour tâter et reconnoître les corps qui se reucontrent sur leur passage.

TENTACULÉ. Muni, armế de tentacules.

TENTACULIFORME. En forme de tentacules, fait camme des tentacules.

TERMINAI. Une bouche est terminale lorsqu'elle est placée à la partie antérieure da corps de l'animal; les tarés ont une bouche terminale.

TERRESTRE. De la terre, qui appaztient à la terre; il v a des coquilles terrestres comme il y en a de marines et de fluviatiles: les limaçons, beaucaup de bulimes, les testacelles. sont des coquilies, terrestres, dont les mollusques, an lieu de vivre dan, les eaux, vivent sur la terre.

TÊT. Revêtement solide et dur, formé de matière calcaire, phs on moins épais, qui constitne les enveloppas on coquilles des mollusques testacés.

TESTACÉ. Couvert, revêtu d'un têt solide et calcaire; sans têt un mollusque est nu, muni d'on têt il est testacé.

THERMAL, THERMALE. Qui appartient aux thermes, aux fontaines d'eau chaude, qui vit dans les eaux chaudes. Dondi Orologio a décrit nn buliue thermal. Il y a des coquilles thermales.

TIGE. Fût, hampe; la tige de l'arrosoir.

TORS. Qui est tordn, qui est formé en colonne torse; la columelle des turbinelles est torse.

TORDU, Qui est formé en colonne torse.

Tordu. Contourvé, déformé; le dévidoir est tordn.

TORSION. Conformation torse on tordue; accident qui a déformé une coquille en forçant le mollnsque qui l'habitoit à s'écarter: des lois ordinaires.

TOLR. Tour despire, une seule circonrolntion de la spire mais complète, un senl tour : dans les nautiles le dernier tour de spire enveloppe tons les autres.

TRANCHANT. Coupant, à vive arête; les côtes tranchanies sont l'opposé des côtes émoussées; la conque cxotique porte des côtes tranchantes. 
TRANSSUDATION. Excrition, suintement qui sort ou trans. sude du mantean et du corps des mollusques; daus les mollusques testacés coute excrétion est calcaire, et éest clle qui par des conches successives forme le têt de ces animaux.

TRANSSUDEli. I'action de la transsudation; le pied des mollusques qui ont un opereule corné, transsude de la matiére cornée.

TRANSVERSE. En travers; le cône flamboyant est orné de deux larges raies brunes et transverses.

TRIANGULAIRE. lait en triangle, ofirant la forme d'un triangle.

TRICUSPJJÉ. Armá de trois pointes conrtes et roisines, disposées en fourche; l'hyale est une conuille tricuspictée.

TRIFIDE. Fendu en trois. Une dent trifide.

TRILOBÉ, Ayant trois lobes.

TROMPE. Esprece de suçoir retractile qui sert de bouche à quelques espèces de mollusques.

TRONCATLRE. Endroit trongué, coupé net et carrément.

TIKOYQUÉ. Coupé, tranché net et carrément; les valves de $l a$ ligule sont tronquées antérieurement.

TLBBE. Гиyau formé soit par la coquille clle-nême, soit par le manteau de l'animal.

Tube. Pied fait en tuyau, tube sur lequel sont portés les anatifes, les térébratules et les lingules.

TUBERCULE. Elévation, petite bosse arrondic, protubérance, mamelon; quelques pourpres sont charécés de tubercules.

TUBFRcULli. Qui a, qui est chargé, garni de tubercules.

TUBERCULIUUX. Ayant des tubercules.

TUBERECX. Chargéde petiles éminences rondes, failes en forme de trufies, de pommes.

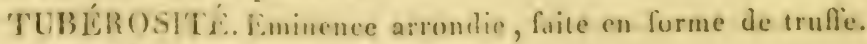
TUBULÉ. lait, formé en tube, ayant un tube.

TUBLTLEUX. En tube.

'TLILE. Chargé d'éailies en forme de tuiles ; le ridacne fuilière est tuilée. 
TUILEUX. Chargé de tuiles.

TURBINE. Contourné en spirale montante ou allongée, formant la toupie; les sabots, les toupies sunt des coquilles turbinées.

TURRICULÉ. Fait en tour, en spire très-allongée, ćlevé en forme de clocher.

\section{U.}

UNGUICULÉ. Fait en ongle; les tuiles ou écailles de la faitière sont unguiculées.

UNIL ATERAL. N'ayant qu'un seul côté.

UNILOCULAIRE. A une seule valve sans cloisons, ni séparations, d'une seule pièce; les cônes, les olives, les strombes sont descoquilles uniloculaires. Ce mot ne se dit que des coquilles uniralves quine sont point chambrées.

UNIVALVE. N'ayant qu'une seule valve, une seule pièce testacée. Coquille univalve.

\section{V.}

VALVE. Pièce testacée qu'on est conrenu de nommer coquille, les coquilles sont formées par les mollusques testacés; une ancienne division les séparoit en coquilles univalves, ou à unc senle pièce; bivalves, on à deux pièces; multivalves, on à plusicurs pièces : nous y avons joint la dénomination de dissivalves, ou à plusieurs pièces, mais qui ne sont pas réunies entre elles par un lien commun, ou par des ligamens ou nerfs.

VARICES. Veines élevées et rameuses, ou bourrelets trèsrenflés ct fort apparens ; les culottes de Suisse sont remarquables parleurs rarices.

VARIQUEUX. Clargé de rarices, de bourrelets renfés, de reines très-apparentes et ramenses.

VARIE. Lorsqu'une coquille présente plusieurs couleurs, sa robe est variée. 
YARIETĹ. Légre changeuent de couleur, de grandeur, ou d'épasseur yur prósentent les mêmes espèces de coquilles, mais prowenant de pay's diveri, onsouvent du móme endroit; rien n’est ansui varié que le limacun des bois, connu sous le nom de liurée; les uns ont des coquilles suns bande, d'antres arec une, deux, trois, yautre et cinq bandes : ce sont autant de variétés.

VASCIULELX. Formé de vajseaux, de conduits, de tayaux aspiratenrs on excrétems.

VENIUUSE. Esprece de godet musculcur on cartilarinenx dont les bras de quelyues mollesplics sout zarnis; les siches, les poulines, le's calmars ont de semblables ventouses : clles lemp serreut à saisir leur proie, et pour s'atlacher aux corps solides.

Vliv'TRAL. Du ventre, voisin durentre; les limaces, les limaçons rampent sur un disque reneral.

VI.VTRE. Partie la plus renliée d'une coquille unisalve; le ventre de la harpe est sa partie la plus renfée.

VEVTRU. Renllé en forme de ventre; les tonnes sont des coquilles globuleuses et ventrues.

VERMICLLAIRE. Fait, formé en façon de ver; les serpules sont des coquilles vermiculaires.

VERMIFORME. Ayant la forme de rer, comme les serpules, les siliquaires et les tarets.

VERSANTE. Une coquille, une ouverture rersantes, sont celles qui teumes horizontalement, la coquille sur le dos, laissent écouler par la base ou canal une partie de l'eau dont on les auroit remplies; toutes les bouches échancrées à la base sont dans cecas.

VERTILAL. En tournaat à cjié; sommet rertical, spire verticale.

V LSICUL IIRE. l'orné en vessie; teansparent, léger, souffé comme unc vessie; los grapnes qui renferment les aufs des calmars sont résiculaires.

VESICULE. Petile ressie; lorsque le jan:hine reut flotter sur lcs eaux, il cufle un amas de résicules.

VIBRATIOY. Mourement rirculare rt trembio:tant quin croboit convulsif; quelques mollusques font vibrer leurs tentacules arec la plus grande rapidité. 
VIBRER. Remuer circulairement et en tremblottant.

VIS. Fait, formé en vis, en spire extrêmement allongée.

VIVIPARE. Qui met au jour ses petits en vie, qui nefait point d'œufs, qui n'est point ovipare; un helix ou limaçon fluviatile porte ce nom, wais c'est une crreur. Les oufs du vivipare sont carrés long, et on peut les tronver sous le maniean de ce mollusque en juillet et août : il est vrai qu'ils éclosent dans la coquille.

VOILE. Large membrane qui s'épanonit an-devant de la tête de quelques mollusques : les nautiles ont une de ces membranes : les argonautes ont deux de leurs pieds munis chacurn d'une voile pareille qui leur sert à voguer.

VOLUTTÉ. Roulé sur soi-même, et renflé du ventre : les trmbales sont volutées.

VOYAGEUR. Mollusque voyageur, coquille royagense: sous cette dénomination on entend ces mollusques qui s'attachent aux pieces de bois flottantes, ou aux animaux qui les transportent au loin: les balanes qui s'attachent anx vaisseaux, et les coronules qui s'implanient sur la peau des baleines sont des mollusques voyageurs.

\section{Z.}

ZOOPHYTE. Nom tiré du grec; il signifie animal plante, il fut anciennement mais improprement appliqué anx coranx, aux éponges, aux flustres et à une foule d'autres productions purement animales, qu'ou regardoit comme autant de plantes mariaes.

ZOOPHYTIQUE. De la nature des zoophytes. 


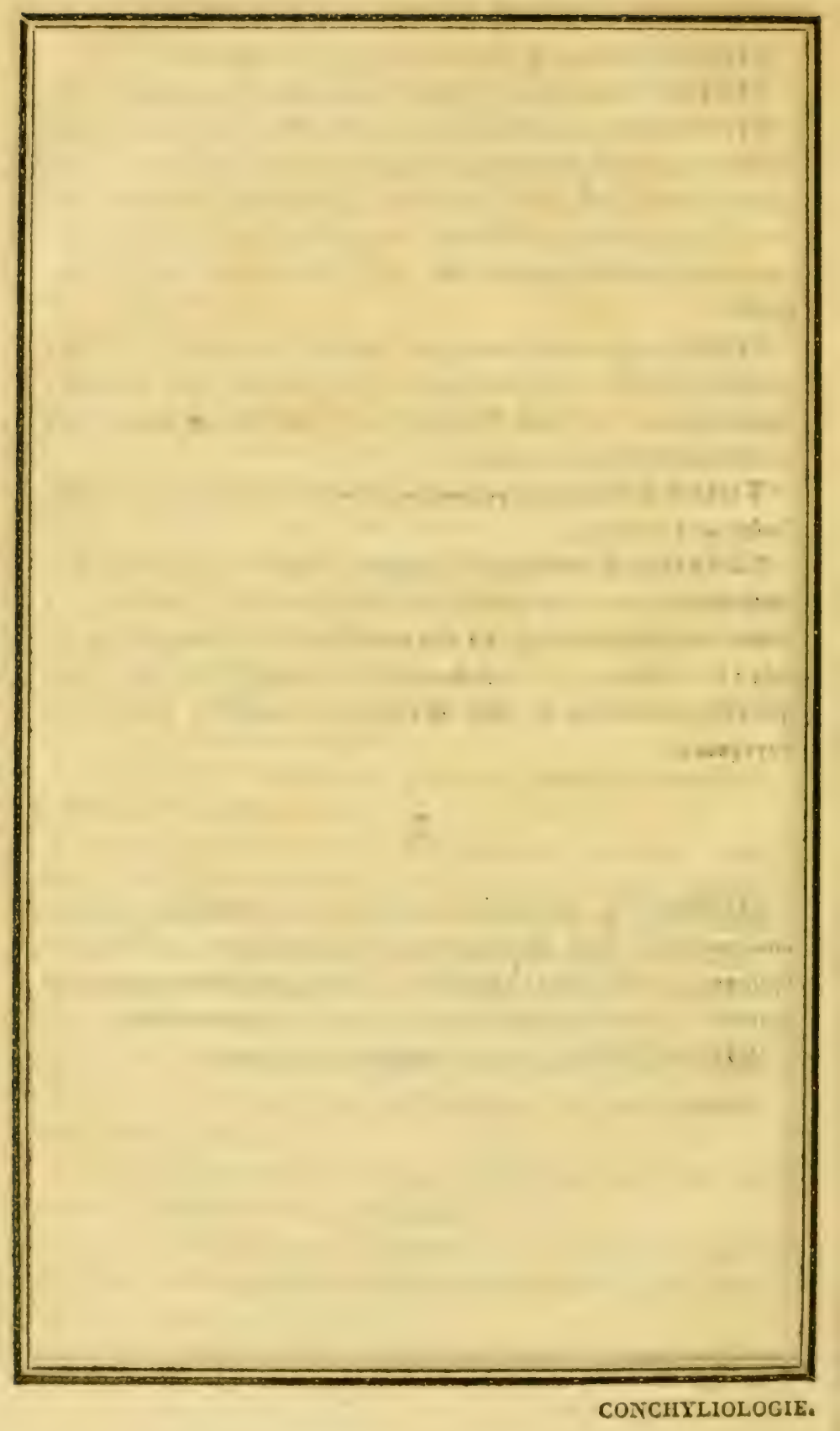




\section{COQUILLES}

UNIVALVES CLOISONNÉES,

CONTOURNÉES EN SPIRALE.

Coquille à spire en disque, sans ombilic. 


\section{PREMIER GENRE.}

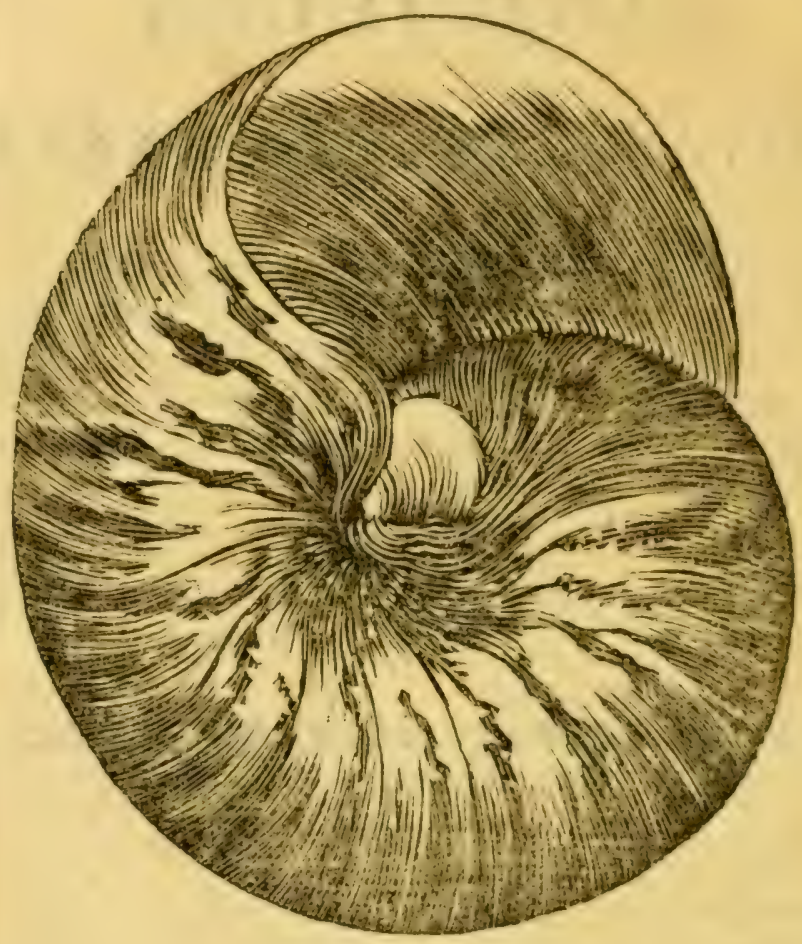

NAUTILE; cn latin NautiLus. 


\section{LE NAUTILE.}

En allemand, Schiffküttel, Fahrküttel, Schiffchen, Schifferchen.

En hollandois, Schipper.

En danois, Skibssnekken.

En suédois, Skeppare.

En anglois, Sailor.

En espagnol, el Nauchel, ô Nautillo.

Caractères génériques. Coquille libre, univalve, cloisonnée, en disque et contournée en spirale aplatie, sans ombilic; le dernier tour de spire renfermant tous les autres; bouche arrondie, évasée, recevant dans son milieu le dos de la coquille; cloisons sans dentelures, et toutes percées dans leur centre par un seul siphon.

\section{Espèce servant de type au genre.}

Le Nautile flambé: Buffon, édition de Sonnini, mollusques, t. 4 , p. 65 , pl. 44 . Ie gros nautile, le nautile fort; le nautile à cloisons; le voilier de différens auteurs. Derschwere oder dicksch: lige nautilus; das dickschalige schiffboot; prlschnecke; perlenmutter-horn; papedasschnecke des Allemands. Nautilus pompilius, nautilus crassus; nautilus major vel maximus; cochlea margaritifera des Latins. Parlemoer nautilus; parlslak, parlemoer-hooren; papedo-hoorn, des Hollandois. Parlunıour schelp, schippertje des Belges. Kika lapia; krany modang des Amboisiens. Bia papeda, bia cojün ; des Malais. 
Circonscrit dians des caractires aussi sévères, le genre des nambiles nous offrira peu d'espéces, car il est enticrement isolí des nautiles ombiliqués : quelques espieces, quclques variétés viennent s'y rattacher; et il en est principilement une qui a des espéces d'oreilles yuion pent voir dans Gualtieri, ind. test. tab. r 8, f. 5 , et tal.. I7, fig. A. Elle a été copiée par Favannes, et elle vient des Malrlives. Diponillís de leur robe jaunâtre et fauve, les nantiles présentent la nacre la plus brillante; gravés et montés sur des pieds d'or et d'argent, ils ont fait un des plus beanx ornemens des buffets anciens. Les nautiles appartiennent aux mers de l'Inde et à celles d'Afrique; l'animal qui les construit se rapproche beaucoup des poulpes, mais il a un plus grand nombre de bras, et dans cette espéce aussi les sexes sont séparés et isolés; jamais il ne vient sur le rivage, il habite au fond des mers, ou vogue à leur surface.

Les nautiles sont assez rares à l'état fossile, ils portent alors les noms de nautilites, en francois; de nautili petrefacli, naulaliti, nauticien latin; de nautiliten, verstcinerte schiffsbote, fahrkiultelstcine, en allemand. Nous en avons trouvéa Courtagnon pris de Rheims, et à Grignon prés Versailles, ayant encore conservé leur nacre: on cn rencontre d'autres a l'état de pitrification it Turin en Piémont, à Richmond en An gleterre, a St.-Gilles dans les Pays-lias, au Havre, a Rouen, pris de Caen, au mont de la Lume en Champagne, a Châlillon en Dauphiné, en Boulommois, a Montbard en Bourgogne, à St-Didier, à Namur, a Nenfchitul, a Boetstein en Suisse, au duché de Weimar, is Aludorff, it Penmling prés Nuremberg en Allemagnc. 
Coquille à spire en disque, sans ombilic. 


\section{GENRE.}

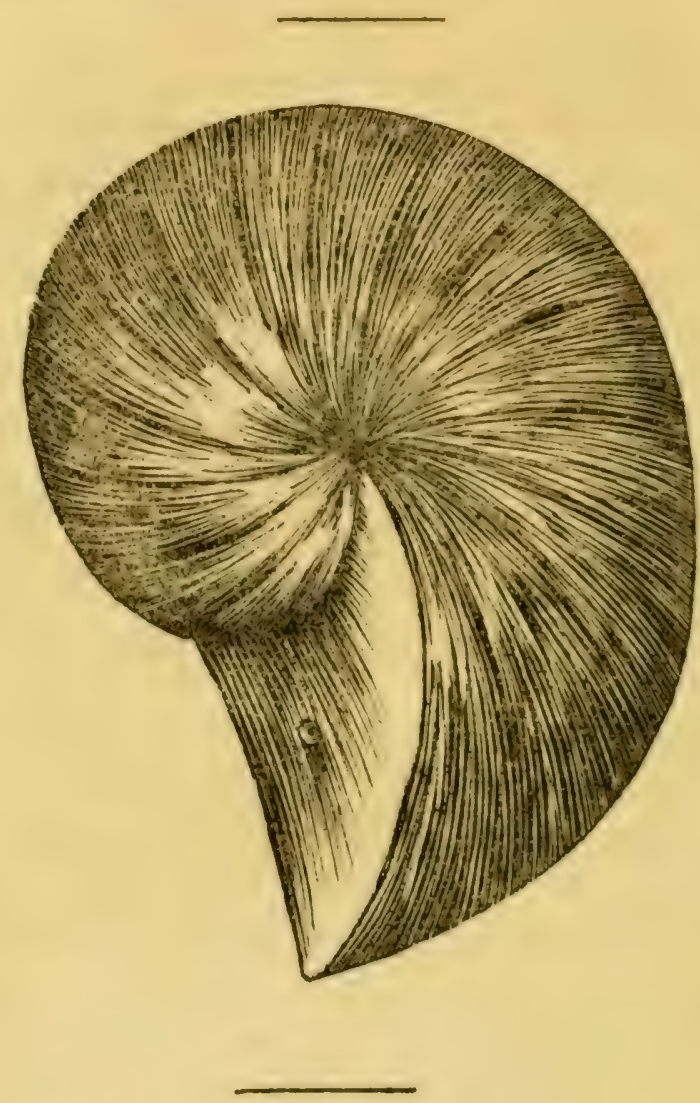

ANGULITHE; en latin, Angemithes. 


\section{L'A N GULITHE.}

Caractères génériques. Coquille libre, univalve, cloisonnée, en disque, et contournée en spirale aplatie, sans ombilic; le dernier tour de spire renfermant tous les autres; bouche triangulaire, recevant dans son milieu le dos de la coquille; cloisons unies, et toutes percées dans leur centre par un seul trou.

\section{Espèce servant de type au genre.}

Nautilite triangulaire du Havre. Histoire des. Mollusques, Buffon, édition de Sonnini, tom. 4, pag. 292, planche 49. Langius, hist. lap. figurat. pag. 91. tab. 25. L. B. Nautilites triangularis. Drykantig versteind schippertje des Belges.

Nous avons recueilli cet angulithe aux pieds des rochers du Havre en Normandie, rochers qui renferment tant de pétrifications; ils sont en grande partie argileux, et la mer dans son flux et reflux, en les sapant par le pied, les dégrade continuellement, elle lave les débris qu'elle en arrache, et lespierres ainsi que les pétrifications restent. Nous ne connoissons pas encore l'analogue vivant de cette coquille; la pétrification dont nous parlons est de couleur grise, et sa matière est argilo-calcaire. On trouve dans quelques auteurs plusieurs coquilles pétrifiées qui se rapportent à notre genre; mais en général ce sont des coquilles microscopiques, Lang cependant publia la figure d'un de ces corps pétrifiés; il est de la grandeur d'une pièce de six sous, celui que nous don- 
unns est de grandeur naturelle; il en est mème de plus grands; car nous en avons possédí des cloisons délachées qui avoient trois fois la mème grandeur. En général tous les auteurs confondirent les angulithes aver, les aunnonites, ou cornes d'Ammon; il en étoit de même avec les natutilites arant que nous les en eussions séparr's dans notre: ourrage faisant suite aux œuvres de Buffon, et cepeurlant comme les nautiles, les angulithes ne prisentent i lextérieur aucun vestigge d'ombilic. Isamark et Bosc, d'apres lui, en firent des orbulithes; plus tard, et dans louvrage que nous venons de citer, nous les joignimes aux nautiles, arec qui ils avoient plus d'analogie. La science conchyliologique étant maintenant plus avancée, leur forne carénée, et leur bouche triangulaire nous ont permis d'en former aujourd'hui un genre particulier.

Celui qui nous sert de type a six pouces de diamitre sur deux pouces et demi d'ouverture à la bouche; le siphon est central, et les cloisons tris-concares, les bords en sont unis, le têt a disparu et les concamérations ont été remplies par une matière crayeuse, calcaire, fortement argileuse et grisatre, de la consistance du marbre. 
Coquille à spire en disque, sans ombilic. 


\section{GENRE.}

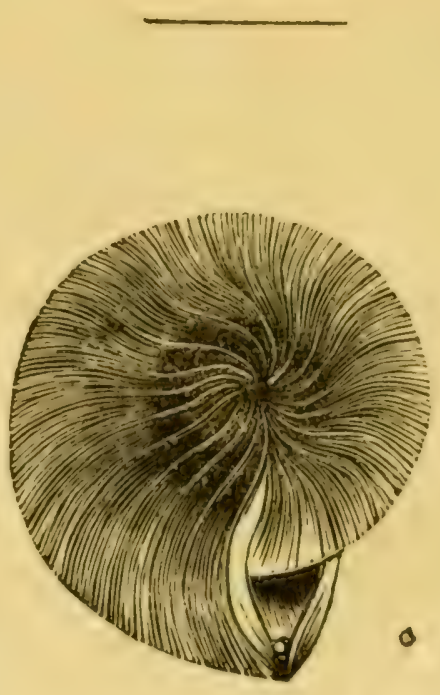

PIIONEME; cn latin Pronenus. 


\section{LE PHONEME.}

Caractères génériques. Coquille libre, univalve, cloisonnée, en disque et contournée en spirale aplatie, sans ombilic; le dernier tour de spire renfermant tous les autres; bouche triangulaire, en partie recouverte sur les bords par une lame, et ouverte au centre, recevant dans son milieu le dos de la coquille; cloisons unies et toutes percées vers le dos d'un seul trou, ou siphon.

\section{Espèce servant de type au genre.}

Le phonème tranchant. Nautilus vortex; en allemand Wirbelschiffer; testac. microsc. a Leo. von Fichtel et J. P. C. von Moll. pag. 33, tab. 2, fig. d - i.

Soldani. Sag. oritt. pag. 99, tab. i, fig. I 2.

- Testaceo. tom. 1 , part. 1 , pag. 66, tab. 59 , fig. t. t.

L'espèce que nous citons demande à être vue à la loupe, elle est de la grosseur d'une graine de navet; on la trouve en très-grande quantité dansles sables littoraux de quelques rivages de la mer Adriatique, et aussi dans ceux de la Coroncine, près de la ville de Sienne, dans l'ancienne Toscane, aujourd'hui Étrurie; sa couleur est blanc-perlé, le plus souvent elle n'a qu'une demiligne de diamètre. On la rencontre encore avec quelques autres qui lui sont congénères, sur les coralines de la Corse, et surtout dans les touffes de celles qui sont formées en buisson.

Nous sera-t-il permis de répéter ici que les coquilles 
microscopiques jouent 1 un tries-grand rúle dans la formation des bancs calcaires; que des chaines entieres des montagnes A pemnines en sont formćes, et qu'à elles seules elles constituent une foule de banes calcaires ou méme on ne les soupconneroit pas?

Le phon'me est arrondi en orhe assez épais, les côtés de la spire se renflent en cóne trìs-obtus, les cloisons sont bleuâtres et apparentes par la transparence de la coquille, elles sont très-courbées, en spirale et presque semi-circulaires: la bouche recouverte en partie, nous conduit naturellement aux coquilles sans ombilic dont la bouche est entièrement recouverte. 
Coquille à spire en disque, sans ombilic. 


\section{IVe. GENRE.}

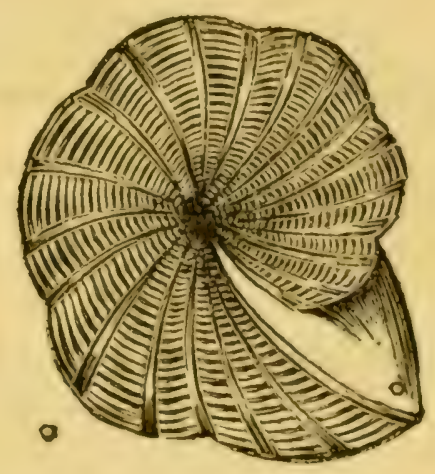

ELPHIDE; en latin ELPmoivir. 


\section{L'ELPHIDE.}

Caractères génériques. Coquille libre, univalve, cloisonnée, en disque, et contournée en spirale aplatie ; sans ombilic; le dernier tour de spire renfermant tous les autres; bouche triangulaire, scellée et recouverte par un diaphragme, recevant dans son milieu le dos de la coquille, et percé à l'angle extérieur par un trou qui se répète dans chaque cloison intérieure. Cloisons unies.

\section{Espèce servant de type au genre.}

L'Elphide souflé, Nautilus macellus; en allemand, der magere schiffer; testac. microsc. a Leo. von Fichtel et J. P. C. von Moll, pag. 68. tab. 10. h. i. k.

Cette espèce, tantôt rosée et $\operatorname{tar}^{\text {' }}$ it de couleur jaunâtre, se trouve assez abondamment dans les éponges, les polypiers, et autres concrétions zoophytiques de la mer Méditerranée : son diamètre est d'un quart de ligne; von Fichtel et von IMoll sont les premiers auteurs qui en aient donné la figure et la description, et quoiqu'ils n'aient apperçu qu'un seul trou sur le diaphragme de cette coquille, c'est cependant par erreur, et sur un simple soupcon qu'il devoity en exister plusieurs autres, qu'ils se sont décidés dans leur ourrage, d'ailleurs si exact, de ne faire qu'une simple variété du genre actuel en le joignant à une autre espèce à six trous dont nous avons, de même que de celui-ci, fait un genre particulier : genres d'autant plus remarquables qu'ils ne présen- 
tent point de bouche ou d'ouverture proprement dite; cette bouche étant fermée et scellie par un diapliragrme qui est intimement soudé aux bords de la corquille; et malgrél'étonnement ou une pareille organisation devroit nous jeter, les genres de coruilles qui vont suivre serviront successivement d'appui à cette nouvelle observalion en histoire naturelle.

L'elphide est renflée, et chaque cloison renferme l'espace de trois côtes, ce qui forme autant de festons: indication de trois époques quelconques, mais annuelles, dans la vie de ce mollusque. 
Coquille à spire en disque, sans ombilic. 


\section{Ve. G ENRE.}

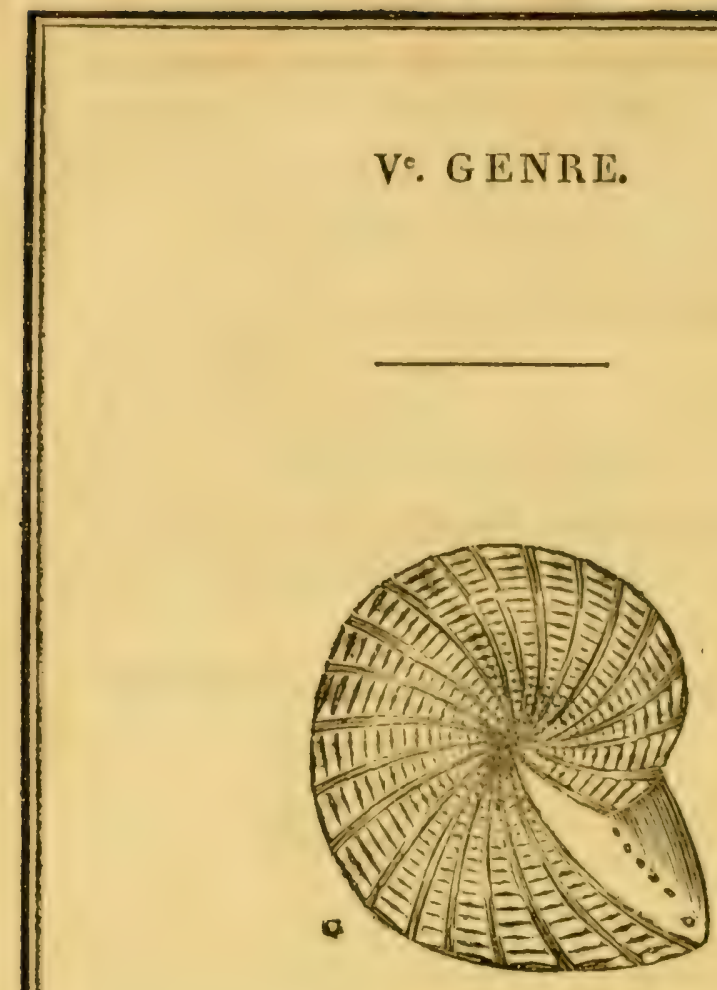




\section{LE GÉOPONE.}

Caractères génériques. Coquille libre, univalve, cloisonnée, en disque, et contournće en spirale aplatie ; mais un peu renflée sur un des côtés; sans ombilic; le dernier tour de spire renfermant tous les autres; dos aigu, bouche triangulaire, scellée et recouverte par un diaphragme percé en longueur par six trous, dont celui de l'angle extérieur est le plus grand, et disposés en série; le diaphragme recevant un peu de côté le dos de la coquille; cloisons unies.

\section{Espèce servant de type au genre.}

Le Géoponejaune. Nautilus macellus; enallemand, der magere schiffer. Testac. microsc. a Leo von Fichtel et J. P. C. von Moll, pag. 66. tab. Io. fig. c. f. g.

Cette espéce si remarquable est de couleur jaunâtre; elle se trouve, comme celle caractéristique du genre précédent, au milieu des polypiers et des concrétions animales, que le vulgaire nomme plantes marines, de la Méditerranée; son diaphragme supérieur est soudé de même, et il ne présente point d'autre ouverture que celle des six trous, sérialement placés dans sa longueur : celui situé à l'angle extérieur est plus grand que les cinq autres, et les deux côtés de la coquille sont inégaux; celui de la gauche est plus bombé que celui de droite, ce qui fait gauchir la bonche dója si 
singulière des coquilles de ce genure; prassitge: aux coupuilles turbinces ou à spire devie, si on. y remarynoit une spire ou un ombilic quelconfue. Le diatmeve du griopone que nutus ávons dessine est dime ligne, il y en a de plus petits. Les auteurs allemands que nous avous cilés, sont les fremices qui nous aient fait connoitre ces corpuille's, ils les rangerene parmi les nautiles. Le diaphragne qui recourre la bouche est un peubombé; elles sont joliment sillonnées et striées. 
Coquille à spire en disque, sains ombilic. 


\section{GENRE.}

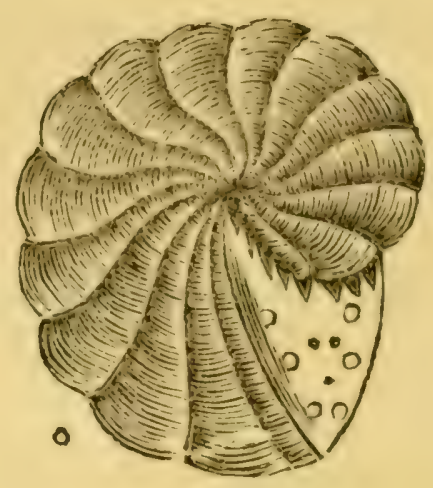

PĹlore; en latin , Pelonus. 


\section{LE PÉLORE.}

Caractères génériques. Coquille libre, univalve, cloisonnée, en disque, et contournée en spirale aplatie; sans ombilic; le dernier tour de spire renfermant tous les autres; dos aigu, bouche triangulaire, scellée et recouverte par un diaphragme recevant dans son milieu le dos de la coquille, percé de six trous, placés sur les bords en deux sćries de trois chacune, renfermant entre eux trois stigmates en triangle, ce diaphragme percé en outre par dix trous en fer de flèche, placés contre le retour de la spire, cinq de chaque côté, et séparés en dents de scie les uns des autres; cloisons unies.

\section{Espèce servant de type au genre.}

Le Pélore ambigu, nautilus ambiguus; en allemand, der zuveydeutige schiffer. Testac. microsc. a lco. von Fichtel et J. P. C. von Moll. , pag. 63. tab. 9. fig. d. e. f.

Cette coquille singulière est originaire du golfe Persique, et de celui de l'Arabie; on l'y trouve principalement dans le sable que contiennent d'autres coquilles plus grandes, abandonnées lors de la mort des mollusques qu'elles renfermoient : c'est ainsi que Spengler les avoit recues, et qu'il les envoya à von Fichtel et à von Moll ; il est possible que les trois stigmates du centre du diaphragme, disposés en 
trianghl', soirent encore antant de trous, ou de siphons; peut-ibe cependant nes sont - ce yne de simples impresions, comm: nous les retrouverons encore dians les loges et dans les diapliragnes des hippurites. Nous navons ponent eu loccasion d'examiner par nousmines les copuilles de ce geme, anssi rare que curieux.

Ix: prilose ambigu ent de couleur blanche, jolinent rose el armé, son diamitre est d'une ligne; il est fresifue oidsiculaire, le centre en est un peu diprime: et le dos aigu. 


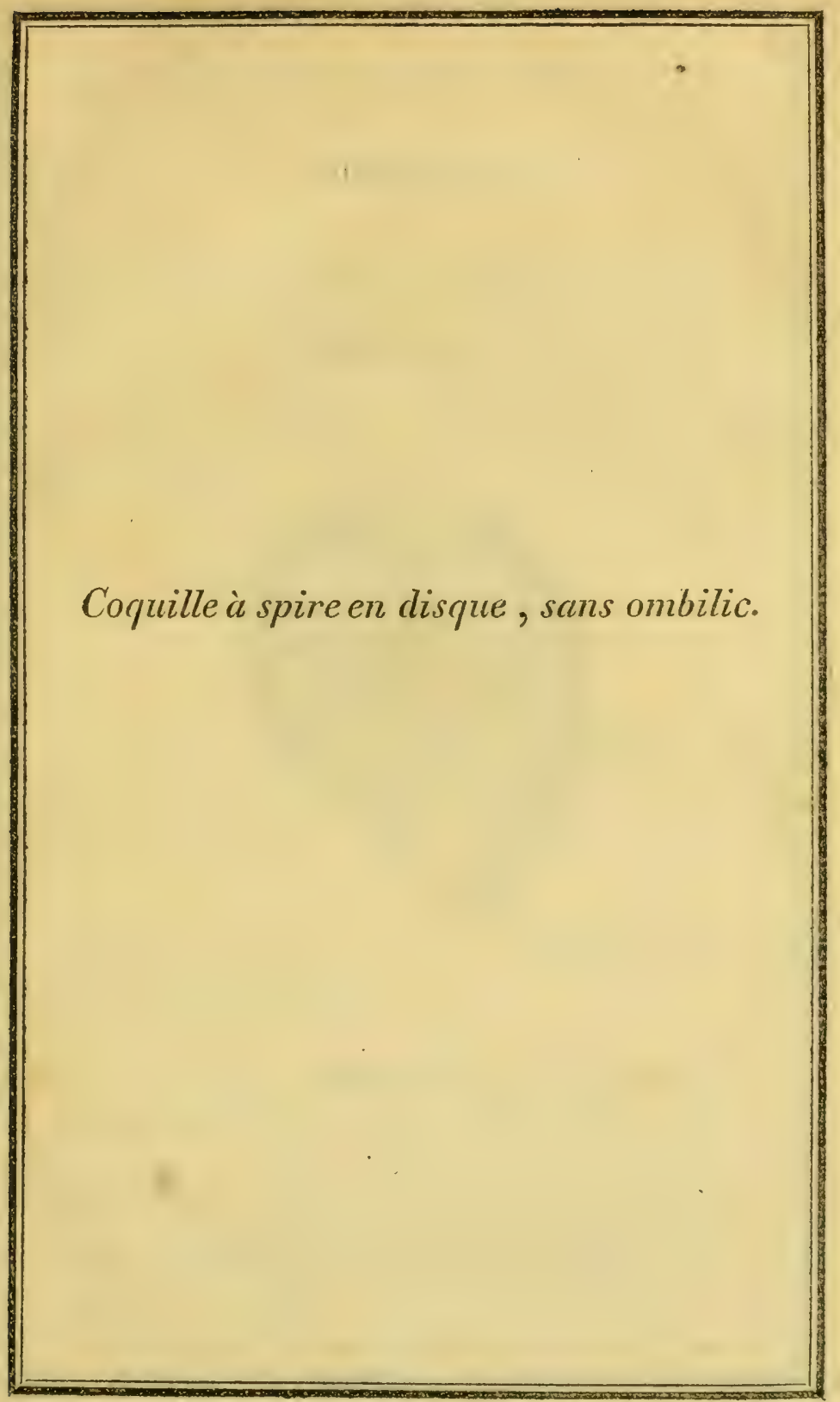


VII. GENRE.

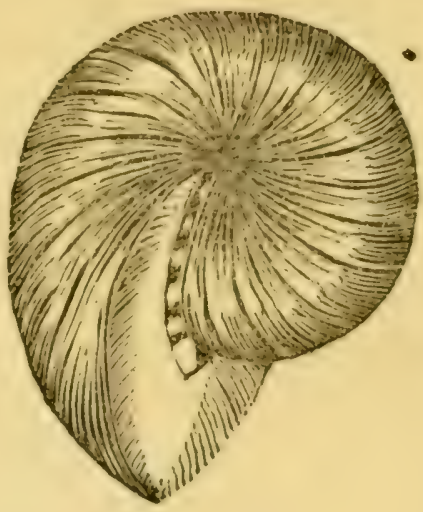

CHRYSOLE; en latin, Cunrsor.cs. 


\section{LE CHRYSOLE.}

Caractères génériques. Coquille libre, univalve, cloisonnée, en disque, et contournée en spirale aplatie; sans ombilic; le dernier tour de spire renfermant tous les autres; dos aigu; bouche triangulairc élargie et scellée, recouverte par un diaphragme reccvant dans son milieu le retour de la spire, plein, sans trous ou siphons, mais crénelé contre ce même retour de spire, et bombé; cloisons unies.

\section{Espèce servant de type au genre.}

Le Chrysole perlé. Nautilus crepidula. En allemand, der pantoffelcehnliche schiffer. Testac, microsc. a Leo von Fichtel et J. P. C. von Moll, pag. x 07. tab. I9. fig. g. h. j.

Soldani, testac. tom. I, part. I , pag. 64 , tab. 58, fig. bb. Nautilus lituitatus.

Les tours de spire du chrysole se renflent, ce qui rend le triangle de la bouche plus évasé que dans les autres genres que nous venons de citer; sa couleur, apres la mort de l'animal qu'il renfermoit, est d'un blanc de perde, mais pendant sa vic la couleur de la coquille est rase; on en trouve pendant toute l'annéc sur les riwayse voisins du port de Livourne dans la ci-devane Tosmane, 
son diametre est de deux tices le: ligne. Les crén. lures yui, dams ce genre, arment le diaphluragme contre le retour de lia spire, sont d'autant plus remarfuables, qu'elles nous conduisent anx camérines et aux antres geures de roquilles nom-coulement dramInries, mais cucore cellulies, comme le sont les canrirines; tant il est rai fue lat nature semisle: avoir ipuise: toutes les formes, particulierenent en cllesmimes, dans leur essence, mais encore dans toutes leurs modifications.

Le cluysole perlé est pour ainsi dire pellucide; il forme une esfrece d'ovale assez allongé. 
Coquille à spire en disque, sans ombilic. 


\section{GENRE。}

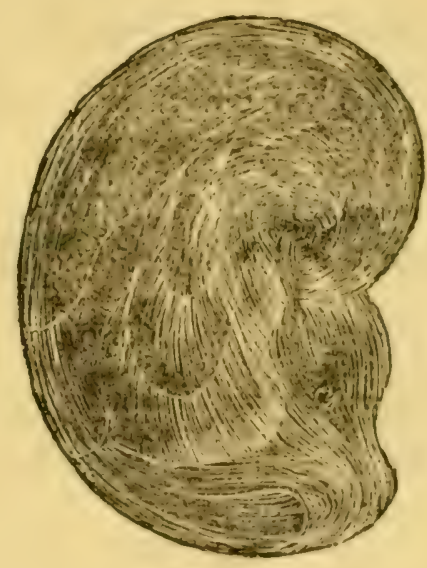

AGANIDE; en latin, Agavides. 


\section{L'AGANIDE, (ou Aganimithe.)}

Caractères génériques. Coquille libre, univalve, cloisonnée, en disque, et contournée en spirale arrondie; sans ombilic; le dernier tour de spire renfermant tous les autres; bouche arrondie, évasée, recevant dans son milicu le retour de la spire; cloisons percées par un seul trou, feuilletées, lobées, en zigzag, ou découpées.

\section{Espèce seruant de type au genre.}

Aganide encapuchonné. Le nautile encapuchonné. Buffon. hist. des moll. édit. de Sonnini, tom. 4, pag. 223 , pl. $4^{8}$, fig. I.

Cette coquille, que nous ne connoissons pas encore à l'état vivant, mais seulement à celui fossile, a le dos rond, et elle est aplatie en façon de palet ; c'est un nautile au premier aspect, mais ses cloisons sont onduleuses, et comme encapuchonnées les unes dans les autres; ces sinuosités conduisent à celles des ammonites à cloisons plus ou moins découpées, mais ici point d'ombilic, et par conséquent elle tient au nautile ou nautilite. Nous l'avons ramassée dans les 
rothers de pierre calcare, noire, fülide ( lipis suillus, pierre de pore) des environs de Namur : sil substance eat trés-spothingue, dure comme le marbre; clle est de consur brune, tirant sur le canclle, et n’a plus son tèl extérienr : mais les concancrations remplies de matièes calcaires, carbonces et solides, ont conserve leur figure primitive. 
Coquille à spire en disque, sans ombilic. 


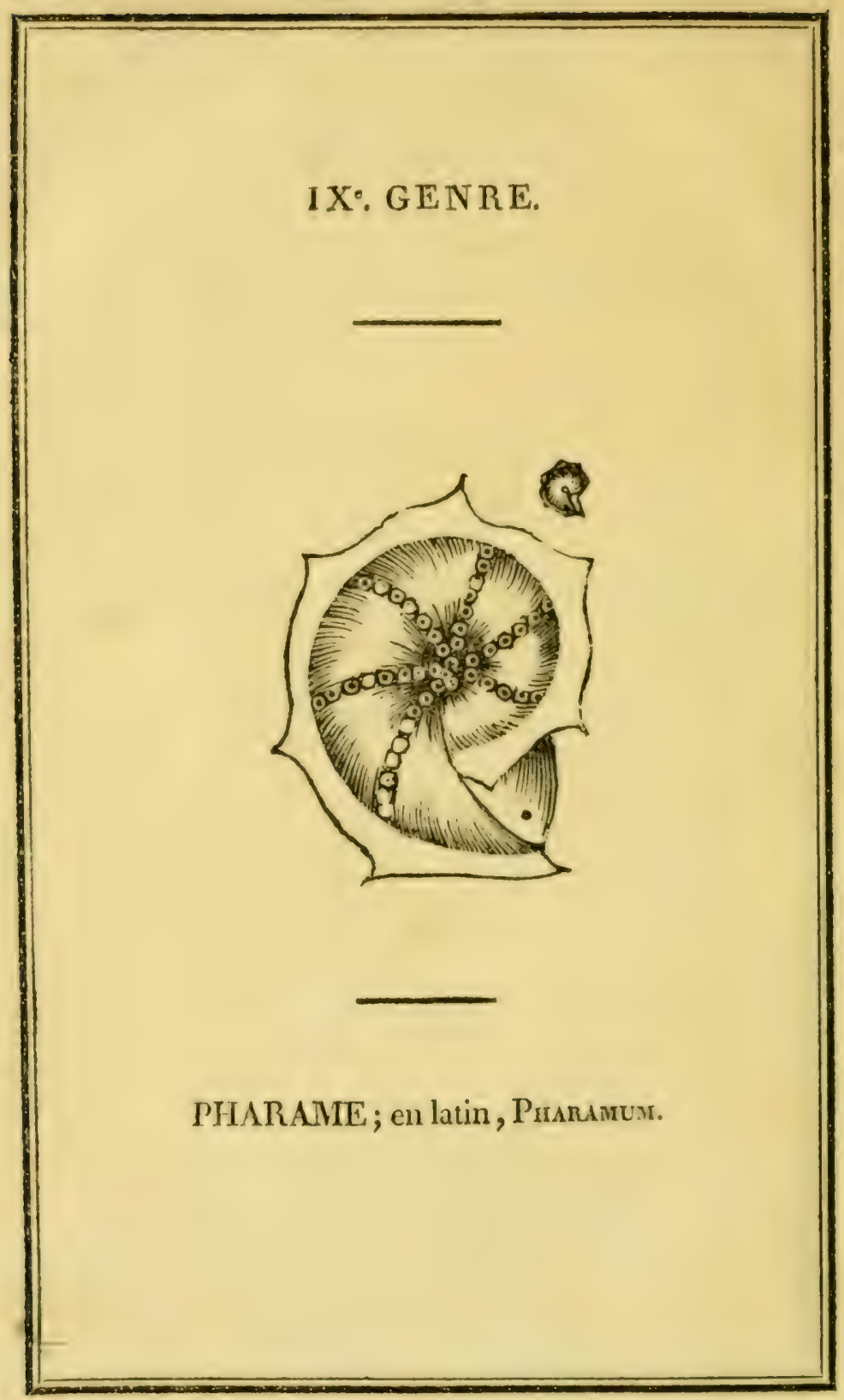




\section{LE PHARAME.}

Caractères génériques. Coquille libre, univalve, cloisonnée, en disque, et contournée en spire aplatie; sans ombilic; le dernier tour de spire renfermant tous les autres, dos aigu, caréné en molette d'éperon ; bouche triangulaire, ouverte, recevant dans son milieu le retour de la spire; cloisons perforées d'un trou à l'angle extérieur, unies et sans ondulations.

\section{Espèce servant de type au genre.}

Le Pharame perlé. Nautilus calcar. En allemand, der spornschiffer. Testac. microsc. a Leo. von Fichtel et J. P. C. von Moll, varietas 4. pag. $7^{3}$. tab. I I. fig. i. k. Linnæus, syst. nat. edit. XII. pag. I 16a. sp. 274.

$$
\text { - XIII. pag. } 3370 . \text { sp. } 2 .
$$

Schrœter, Einleit. in die Conch. kennt.I. B. d.pag. g.II. Sihreiber, Conch. Kennt. I. Bd. pag. 2. II.

D'après les apparences et l'aspect de cette coquille, de la carène et de son armature, tous les auteurs sans exception qui traitèrent des coquilles microscopiques en ont confondu une foule les unes dans les autres, sans faire attention à la différence de leurs bouches, ou à d'autres caractères très-distinctifs. Dans l'ouvrage, d'ailleurs si estimable de von Fichtel et von Moll, trois planches entières, celles I r , х 2 et I 5 , sont consacrées à plusicurs coquilles qui ne sont rien moins que congénères, et que ces auteur's ne donnent que comme 
des variétís, tamulis que toutes dillérent, fine quelquesunes somt des espieces particulieres, et que les autres fornent des genres : ils disent mêne que leurs varivés sont si multiplices quil leur paroit presque impossible de pouroir leur assigner un caractire spécilinge. Nous mpirons itre plus heurcux on les classant succescivenient iz leurs genres, déterminés d'aprés la forme de leur bouche, et d'aprés quelifu'autre différence notabl. ct caractéristique. Dans le greme dont nous traitons, la coquille qui mous sert de type, a naturellement la grandeur d'ume assez forte lentille, c’est-a dire i-peu-pres trois lignes de diamitre; nous ne domnons point comme un caractère génćrique les grains dont. les bords de ses cloisnus sont perlés; ils constituent senlement l'espérs. Sa conleur est blenatre et irisce; à l'éat fossile, les memes corpuilles sont brunes et rongeitres: on les trouve dans la mer Adriatique, principalement sur ses bords pris de Rimini; ct quant auxfossiles, a la Coronrine pris de Sicme en Toscanc. Dans les mimes cmlroits on en rencontre quelques autres esprees, yuan premier abord on confondroit arec celle.ci; mais ches mi lourerture du siphon faite en rerrue, ou radice. In bouche du pharame perlé est ourerte, comme celle des nautiles. 
Coquille à spire en disque, sans ombitic. 


\section{$\mathrm{X} \cdot$ GENRE.}

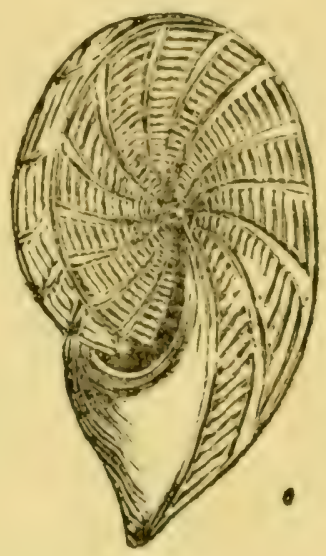

ANDROMlDE; cn latin, Axpronedes. 


\section{L'ANDROMÉDE.}

Caractères génériques. Coquille libre, univalve, cloisonnée, en disque, et contoumée en spirale arrondie; sans ombilic, Ie dernier tour de spire renfermant tous les autres; bouche triangulaire, faite en forme de poire, recevant dans son milicu le retour de la spire, et recouverte par un diaphragme offrant une ouvcrture sémi-lunaire contre le retour de la spire; dos caréné; cloisons unies.

\section{Espèce servant de type au genre.}

Andromède gauffrée. Nautilus strigillatus, en allemand der gestrichelte schiffer. Testac. micros . a Leo. von Fichtel et P. J. P. C. von Moll. pag. 49. tab. 5. fig. c. d. e.

Soldani testac. T. I. P. I. pag. 54. tab. 34. fig. I ?

Quoique dans le dessin cette coquille paroisse elliptique, elle est parfaitement ronde et assez renflée : on pourroit, à la rigueur, y recormoître une espèce d'ombilic, mais il est si peu marqué, si peu apparent que constamment il seroit contesté. Ces apparences sont formées par un léger creux central d'ou ces cloisons partent en rayonnant; les 
coicons sont tris-apparentes el assez rapprochices les unes des autres.

La conleur de l'andromede gaulfree est irisér : on la trouve en quantité a Poville pres de Nori, sur les bords de: la mer Adratique: la coquill. a une demi-ligne de diantide, et plusicurs espéres viemnent se ratlachex a ce genre; le diaphragne de la bouclie est bombé. 
Coquillle à spire en disque, sans ombilic. 
Xie. GENRE.

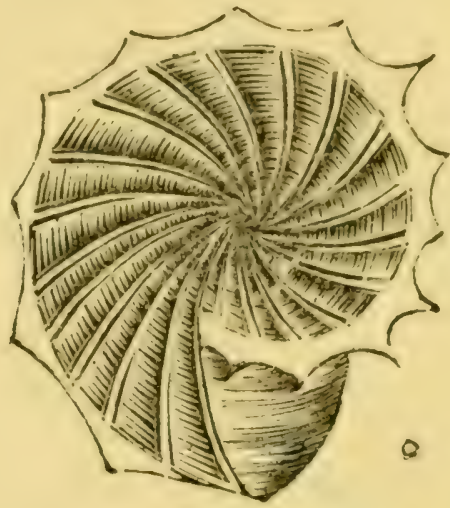

SPORULIE; cn latin, Sponilus. 


\section{LE SPORULIE.}

Caractères génériques. Coquille libre, univalve, cloisonnée, en disque, et contournée en spirale aplatic; sans ombilic ; le dernier tour de spire renfermant tous les autres; bouche ouverte, triangulaire, recevant dans son milieu le retour de la spire; dos caréné et armé; cloisons unies, percées au centre.

\section{Espèce servant de type au genre.}

Sporulie pectiné. Nautilus strigillatus. En allemand, dergestrichelte schiffer. Testac. microsc. a Leo von Fichtel et J. P. C. von Moll. pag. 5o.tab. 5. fig. f. g. deuxième variété.

Dans les coquilles de ce genre, comme dans celles qui constituent celui des pharames, nous avons une preuve nouvelle que la nature n'a laissé aucune place sans chercher à la remplir; les sporulies, comme les pharames, ont une carène armée, éperonnée de forme singulière, espèce d'appendice à leurs coquilles, et qui vient remplir une place qui, sans elle, ne présenteroit qu'une lacune. Les pointes de cette armure sont inégales. 
I a corpuille clu sporulie pectiné, est finement plissée en sens contraire des stries de l'accroissement successif, el par conséfucint des cloisons, et l'melroit de ces cloisons est indiqué par ders espéces de cótes qui partent du centre en divergeant vers la circonférence.

La couleur de cetse conuille est blanchitre, perlíe et irisce; on la trouve en imncuse quantité pries die Novi, sur les plages de la mer Adrialique; clle a une: ligne de diametre. 
Coquille à spire en disque, sans ombilic. 


\section{GENRE.}

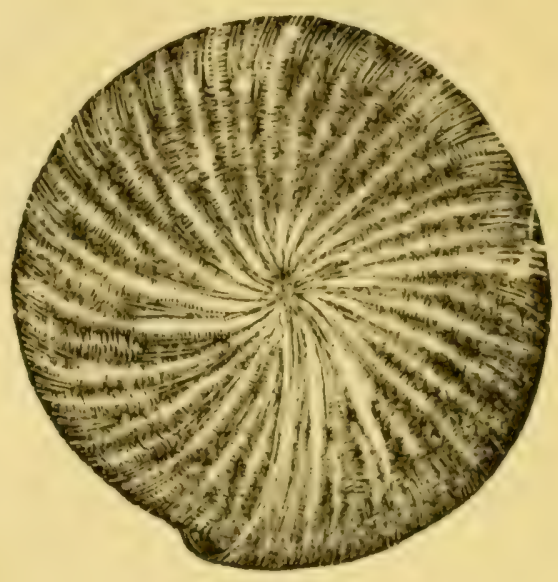

CANTHROPE; en latin, Canthropes. 


\section{LE CANTHROPE.}

Caractères génériques. Coquille libre, univalve, cloisonnée, en disque, et contournée en spirale trèsaplatie; sans ombilic; le dernier tour de spire renfermant tous les autres; bouche arrondie en ferà cheval, recevant dans son milieu le dos de la coquille; cloisons unies et percées, contre le retour de la spire, par un seul siphon ou trou.

\section{Espèce servant de type au genre.}

Canthrope galet. En belge, Plat schippertje.

Cette coquille que nousne connoissons encore qu'à l'état de pétrification, se rapprocheroitinfiniment des nautiles, si ce n'étoit son extrême aplatissement et son siphon placé médiatement contre le retour de la spire, au dos de la coquille : elle est représentéc ici de grandeur naturelle, et telle que nous l'avons trouvée, déjà un peu roulée, parmi les nombreux galets tourmentés par le flux et reflux de la mer sous la tour d'ordre à Boulognesur-mer. Elle ressemble extrêmement par sa forme aux galets; mais sa superficie est ondée par des plis trèsrapprochés les uns des autres; la bouche est très-étroite, car le dernier tour n'a tout au plus qu'une ligne de saillie sur les autres, et par conséquent elle présente la forme d'un fer à cheval très-allongé. 
Sa substance est argilo-calcaire, et elle se rapproche: beaucoup de celle de la pierre puante, lapis suillus.

Nous crnyons que cette espèce est trisrare, n'en avant retrouvé le dessin ni la description dans aucum dess nombreux auteurs que nous arous été à mime de consulter it cet égard; mais il existe quelyues autres pétrilications qu'on peut y rapporter, quoiqu'en général elles soient plus renflées et plus épaisses. 
Coquille à spire en disque, sans ombilic, à ailesétendues. 
XIII'. GENRE.

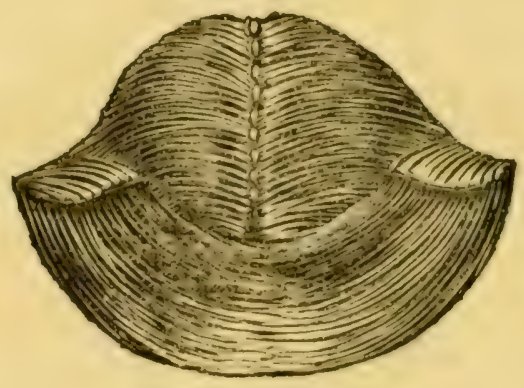

BELLEROPHE; on latin, Bellenopion. 


\section{LE BELLEROPHE.}

Caractères génériques. Coquille libre, univalve, cloisonnée, roulée sur elle-même et en spirale déprimée, formant la navette; le dernier tour de spire renfermant tous les autres; bouche très-ovale, recevant dans son milieu le dos de la coquille; cloisons unies, percées par un siphon.

\section{Espèce servant de type au genre.}

Le Bellérophe vasulite. Vasulite déprimé ; Bellerophon vasulites, Histoire naturelle des Moll. Buffon, édit. de Sonnini. vol. 4. pag. 298. pl.L. fig. 2 et 5. Le nautile déprinué. En hollandois; den gevleugelden nautilus. De Hupsch Naturgeschichte des NiederDeutschlandes, etc. tab. 5. fig. 20 et 2 r, et pag. 27 . Einfacher nautilit von Bensberg, et ibid. fig. 22. Einfacher nautilit von Eiffel.

C'est le baron de Hupsch, naturaliste distingué de Cologne, qui, le premier, publia, dans un petit ouvrage assez rare, deux espèces congénères de cette coquille, très-remarquable par sa forme en navette, par la disposition de sa bouche, et par son évasement; nous ne la connoissons encore qu'à l'état fossile, et M. de Hupsch les avoit rencontrées parmi d'autres pétrifications dans l'Eiffel, canton du duché de Juliers:j’en ai possédé qui avoient trois pouces d'une extrémité à l'autre; la coquille étoit convertie en spath calcaire d'un blanc très-éblouissant, qui tranchoit vivement en onyx sur le marbre noir spathique fétide qui en remplissoit toutes 
les cloisons. D'autres échantillons renfermoient une craie argileuse, happant fortement à la langue.

La bouche des bellérophes s'épanouit en demi-cintre, comme celles des ovules, nommées navettes par les conchyliologues (Bulla birostris. Limn. syst. nat. verm. test. pag. 5425. spec. 5. edit. X). Elle a de même deux pointes ou prolongemens à ses côtés, auxquels on pourroit cependant domer le nom d'oreilles : la coquille est bombée, striée par de petites côtes sillomnées, partant de chaque oreille, pour se rendre au milieu du dos arrondi et remarquable par une espèce de chainette perlée et disposée en série ou cordonnet, qui sépare la coquille en deux parties égales, comme le feroit une carène; le tît a une ligne dans sa plus grande épaisseur. Il est probable que le bellérophe vasulite se trouve aussi dans les environs de Namur.

M. de Hupsch, naturaliste infatigable, aroit recueilli une foule de matériaux en tout genre; nous sarons que son cabinet, si précieux sous tant de rapports, se trouve aujourd'hui dans toute son intégrité a Darmstadt; et nous formons des rœux, afin que le public et la science jouissent enfin, par la publication, de tous les faits que ce savant avoit su réunir avec tant de soins. 
Coquille à spire en disque, sans ombilic. 


\section{XIVe. GENRE.}

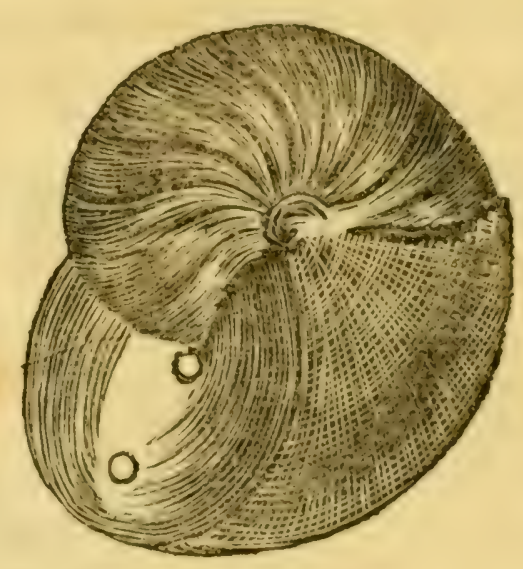

BISIPHITE; en latin, Bisirnytes. 


\section{LE BISIPHITE.}

Caractères génériques. Coquille libre, univalve, cloisonnée, roulée sur elle-mème et en spirale déprimée, le dernier tour de spire renfermant tous les autres; bouche ronde et évasée, recevant dans son milieu et.en retour le dos parfaitement arrondi; cloisons unies percées par deux siphons en ligne droite, placés l'un près du retour de la spire, et l'autre plus à l'extérieur.

Espèce servant de type au genre.

Le Bisiphite quadrille. Bisiphytes reticulatus. Nautile à deux siphons. Hist. nat. des Miollusques. Buffon, édit. de Sonnini, vol. 4. pag. 208. pl. XLVI. fig. 2.

Nous fimes connoitre les premiers cette coquille fossile dans l'ouvrage que nous venons d'indiquer. Cette pétrification venoit de Sombrenon en Bourgogne, elle avoit encore en partie conservé son têt, il étoit assez épais et quadrillé; et les deux siphons séparés et distincts, permettoient le passage à un plomb de chasse, pareil à ceux dont on se sert pour tirer le lièvre. La couleur du têt étoit rousse ct jaunâtre, entièrement calcaire, et répandant sous le marteau une odeur fitide. 11 y en a de plus de deux pieds de diamètre, et dont la coquille a deux lignes d'épaisseur, quadrillée, et ressemblant au plus gros canevas. On en rencontre encore de plus ocracés dans les environs de Bruxelles et aux carrières de marbre noir de Barbançon dans l'enclavement des Ardennes; nous en avons possédé de trois pouces et 
demi de longsur deux d'ouverture; convertisen marbre noir, leurs con amérations offroient des cristallisations spathingues et calcaires, et leur cassure ne rendoit point d'odenr fétide; leur têt ou robe extéricure étoit uni. Ces derniers bisiphites, à qui nous donnerons le nom de flambés, ont dẹja été gravésà l'état marin par Gualtieri (index testaceorum, tab. 18. la vignette fig. 4.), et par Favannes, sous le nom de grand nautile épais à deux siphons (Conch. tom. I. part. 2. pag. 72 f et pl. VII. Lit. D. 5 ; ct zoomorph. pl. LXIX. fig. A. 4. ) Ils sont les analogues virans aujourd'hui dans les mers orientales de ceux que nous retrourons pétrifiés en Europe au milieu de la forèt des Ardennes; leur robe légerement teintéc de safran est flambée de brun dans le genre des taches du grand nautile flambé.

Dans ce genre nous pouvons donc compter trois espéces, dont deux à l'état de pétrification, et la troisicme à l'élat marin, comme à celui fossile. Quoiqu'elles ne soient plus ombiliquées, le centre de leur disque offre une espece de creux, qui nous conduit naturellement aux coquilles contournées, comme le sont les แantiles, mais qui offrent de plus ru'elles un ombilic prononcé : ces coquilles, connues sous le nom de namiles nmbiliqués, nous servent de passage pour atciudre les cornes d'Ammon et les ammonites. 
Coquille à spire en disque, ayant un ombilic. 


\section{$X V^{\circ}$. GENRE。}

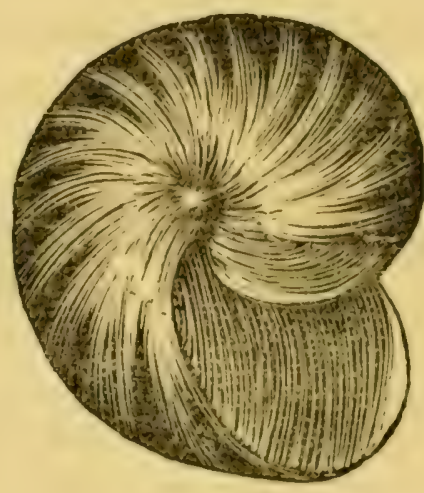

OCEANIE; en latin Oceanus. 


\section{L'OCE ANIE.}

Caractères geénériques. Coquille libre, univalve, cloisonnée, en disque, et contournée en spirale, ayant un ombilic; le dernier tour de spire renfermant tous les autres; bouche arrondie, évasée, recevant dans son milieu le retour de la spire. Cloisons unies et percées parun siphon.

Espèce servant de type au genre.

Océanie flambée. Oceanus flammeus. - Le nautile ombiliqué. Hist. nat. des Mollusques. Buffon, édit. de Sonnini. - Le petit nautile ombiliqué. Favannes, conch. vol. I. part. 2. pag. 725 . pl. VII. Lit. D. I. ; et Zoomorph. pl. LXIX. let. A. 4. - Nautilus crassus minor, concameratus et umbilicatus. Favart d'Herbigny, dict. tom. I I. pag. 418. Het doorgeboord schippertje, des Hollandois.

Nous venons de donner quatorze genres de coquilles que l'on a confondus jusqu'à ce jour sous la dénomination de nautiles; de ces genres aucun n'est ombiliqué, et cependant nous devons avouer que malgré leur grand nombre, il eût peut-être été nécessaire de faire encore quelques coupes; car il existe des nautiles ou nautilites à trois siphons, d'autres à siphon continu, et d'autres encore qui plus d'une fois pourront faire hésiter sur la place ou ils devront venir se ranger. Nous allons maintenant nous occuper de quatre genres, dont l'ancienne nomenclature fit ou auroit fait des nautiles ombiliqués, et auxquels nous avons cru devoir donner enfin un nom géné- 
rique, et qui leur appartint. L'océanie flambé n’a'ordinairement que deux à trois pouces de diametre, et souvent il est bien plus petit, sa robe est blanchûtre, flambée de fauve et de couleur de feu; la nacre intérieure est grise, irisée, réflétante, et du poli le plus doux; la poupe ou retour de la volute est teintée de feuille morte rembrunie.

La corguille est fortement arrondic, presqu'en boule, et l'ouverture est it-peu-pres ronde; clle est munieà son centre d'un ombilic, percé à jour, au travers durjuel on peut fair e passer un fil, caractire inhérent à ce genre. L'océanie flambe vient des Molurques(Valentin amb. cor. univ. (ig. 4), il est assez rare. L'analogue fossile a été publié par Breyn (de polytlı. tal). 2. fig.5.\$58). Les deux Bayer, de Iupsch et Liang ( I) nous en ont donné au même état qui avoient tantôt conservé leurs têts, el qui d'autrefois étoient convertis en pyrites; mais Knorr (2) en a publié un trés grand venant a'Aristorff, dans le canton de Bàle; ilest énorme pour la taille, et cependant l'analogue de l'océanieflambé est originaire d'Amboine, dans la mer des Indes. Il est probable que nous n'en connoissons point encore de grands individus à l'état marin, et vu la rareté des petits, il est à croire qu'ils ne virent qu'à une grande profondeur dans la mer, et que ce sont des coquilles pélagiennes. On en trouve encore de pyritisís et it tèt calcaire à Neufchátel en Suisse.

(1) et (2) Oryct. norica.pl, 11. fig. 8, et pag.3 3. - Ferd. Bayer i usuppl. tab. 10. fig. 3. 4. 5. ct pag. 17. - et iljid. tab. I1. fig. I. 2. et pag. 17. - De llupsch naturgesch. cte. vol. I, tab. 3. fig. 19, et paer. 25, paragraphe 33. - Lang. hist. lap. helr. tab. 22. fig. A, et par. 84. - - Knorr de poly. vol. i I, sect. 1. pag. 44, et pl. A. IV. fig. I. 
Coquille à spire en disque, ayant un ombilic. 


\section{$X V I \% G E N R E$.}

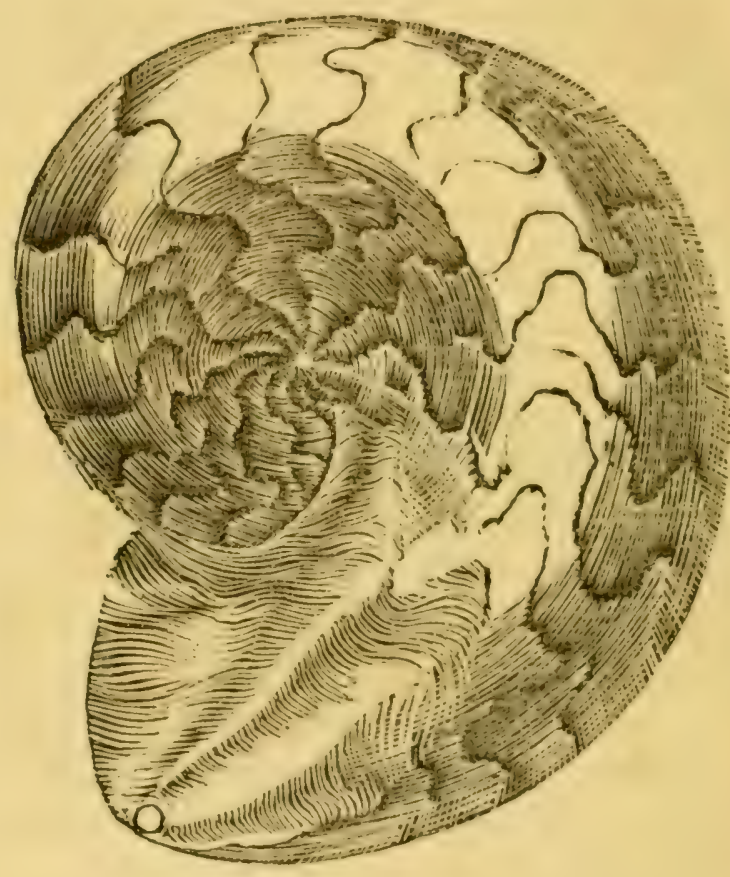

PĹLAgUSE; en latin Pelagus. 


\section{LE PÉLAGUSE.}

Caractères génériques. Coquille libre, univalve, cloisonnée, en disque, et contournée en spirale aplatie; ayant un ombilic; le dernier tour de spire renfermant tous les autres, bouche arrondie, évasée, recevant dans son milieu le retour de la spire; cloisons lobées, persillées, dentelées et percées par un seul trou.

\section{Espèce servant de type au genre.}

Pélaguse lobiserraté; nautilite persillé. Tous les nautilites ou nautiles pétrifiés ont en général été confondus par les anciens oryctologues avec les ammonites ou cornes d'Ammon, et nous croyons être les premiers qui, dans l'histoire naturelle des Mollusques, faisant suite à celle de Buffon, ayons sorti cette matière de l'espèce de chaos où elle étoit ensevelie : dans notre plan actuel, forcés de prendre une nouvelle extension, et de ne donner aux choses que leurs noms rigoureux, nous avons dû séparer des coquilles à ombilic, celles qui n'en avoient aucun, et réunir parmi ces coquilles à ombilic des genres séparés et distincts. Nous venons de voir dans les océanies des cloisons unies et simples; le genre actuel en offre de persillées et de découpées, et c'est ce qui l'en sépare; c'est sous ces derniers que viemnent se ranger tous les ci-devant nautiles dont les cloisons, loin d'être unies, sont lobées, persillées ou dentelées, coquilles dont on retrouve les pétrifications dans une multitude d'auteurs qui traitèrent de ces objets. C'est au genre 
pélaguse que nous rapporterons le natulitic ondulé que nous publiàmes dans l'histoire des Mollusques que nous venons de citer, tome 4, page 247 , planche XLVI, figure 3 , de mème que toutes les antres coquilles qui lui sont congénères. Nous ne comnoissons pas encore de coquille à l'état marin qui puisse être citée comme analogue, et c’est principalement par la déperdition du tết qu'on s'apercoit de la découpure des cloisons. Le pélaguse qui nous sert de type, vient des Vaches noires en Normandic, il est calcaire, et ses cloisons sont pyriteuses; on trouve les pélaguses dans tout état de pétrification, et on en a rencontré dans l'Inde comme en Europe, et il est hors de doute que tût ou tard nous connoitrons enfin cette coquille pélagienne, qui avec d'autres semble n'habiter que le fond des mers les plus profondes. C'est ainsi que l'étude des pétrifications nous mine à la connoissance d'ères que nous dérobent encore, malgré nos recherches, les profondeurs incommensurables de l'océan, et qui chaque jour, ajoutent, lors de leur non-existence, aux couclies de ce globe évidemment formé par les dépouilles des êtres vivans, dont la taiche unique et suprème est de créer quelques parcelles solides qui doirent se réunir, s'ensevelir, s'amalgamer eufin dans les couches terraquées et pressées qui constituent et augmentent depuis des siecles incalculables le globe de la terre. 


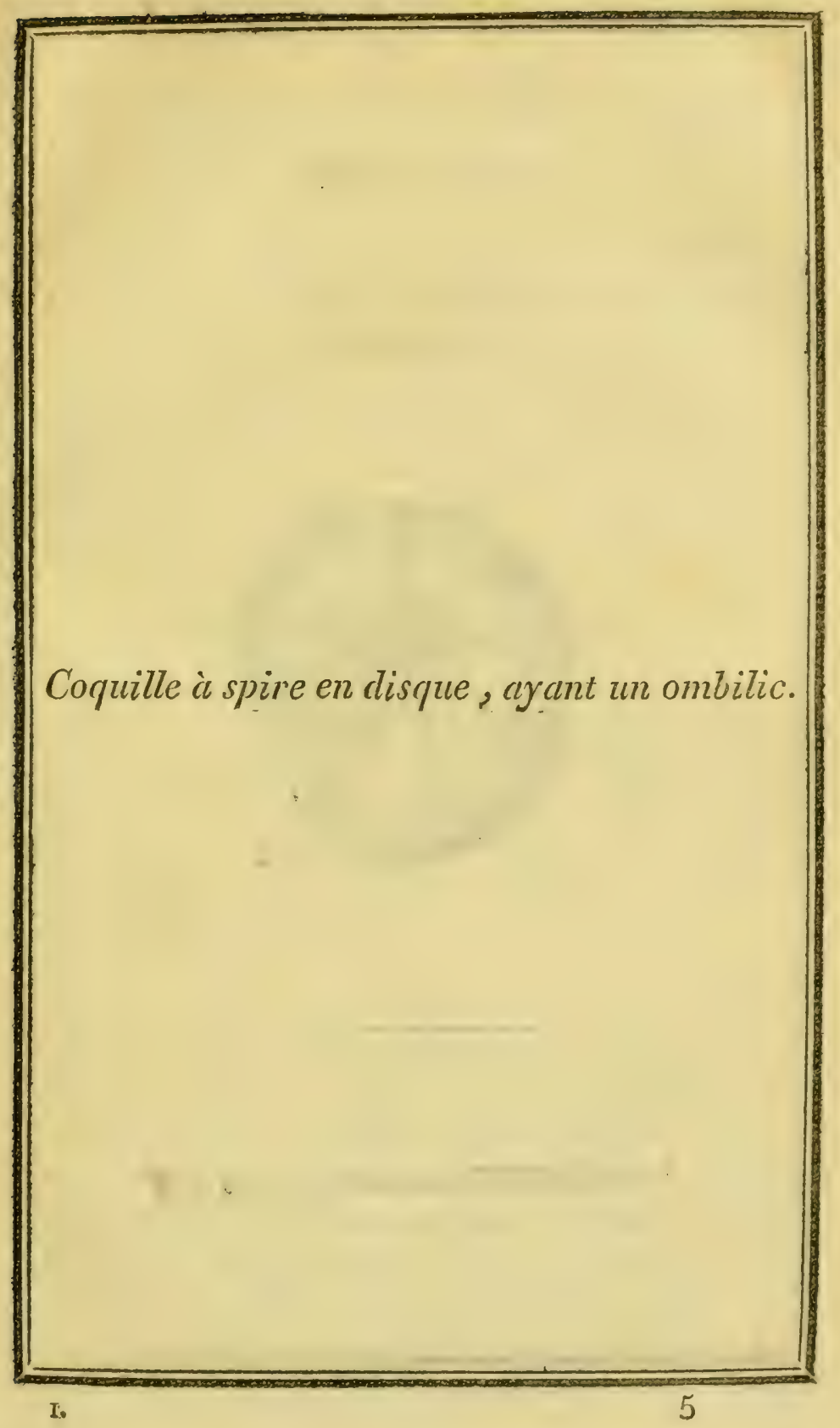



66
COQUILLES.

XVIIE. GENRE.

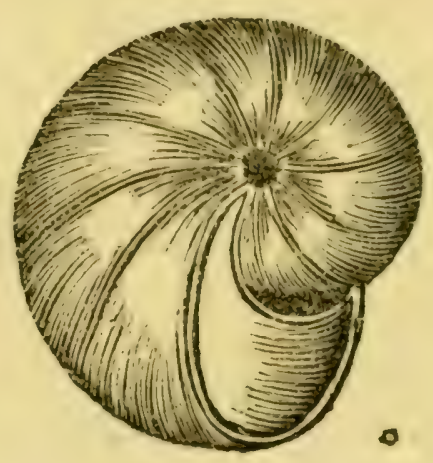

MLLONIE; en latin, MeLons. 


\section{LE MÉLONIE.}

Caractères génériques. Coquille libre, univalve, cloisonnée, en disque, et contournée en spirale aplatie; ayant un ombilic; le dernier tour de spire renfermant tous les autres; bouche arrondie, recevant dans son milieu le retour de la spire, scellée et recouverte par un diaphragme sans siphon, mais laissant une ouverture sémi-lunaire contre le retour de la spire; cloisons unies.

\section{Espèce servant de type au genre.}

Le Mélonie étrusque. Melonis etruscus. - Nautilus pompiloüdes. Der kleine dicke schiffer. Testac. microsc. a Leo. von Fichtel et J.P.C. von Moll, tab. 2, fig. a , b , c. - Nautilus melo. Saggio oritt. pag. 100. tab. 2 , fig. I6. tt. II. VV. XX. - Testaceo. T. I. P. I. pag. 49. tab. 46. fig. qq?

Cette coquille, par ses formes extérieures, ressemble tellement en petit au nautile flambé, qu'au promier abord on pourroit la regarder comme une enveloppe du premier âge de ce mollusque ; mais sa bouche scellée et recouverte, et son ouverture sémi-lunaire démontrent bientôt qu'elle appartient à un genre particulier, dont pour nous elle constituera la tête, à côté de laquelle viendront se ranger ses congénères. Sa couleur est blanche, mais les cloisons se dessinent sur le têt par des 
raies blemitres dans létat virant ou marin ; lorsqu'elles sont fossiles, elles partagent la couleur des lits ou clles sont renfirmées; dans ceux calcaires elles sont grises ou cendrées, dans ceux ferrugineux, jaunâtres et ocracées.

On troure les mélonies a l'état marin sur les marlrépores, el sur les concrétions zoophytiques de la mer. Héditerranéc; et à l'état fossile à la Coroncine en Toscane.

Ces coquilles ont une demi-ligne de diamitre. 
Coquille ì spire en disque, ayant un ombilic. 


\section{GENRE.}

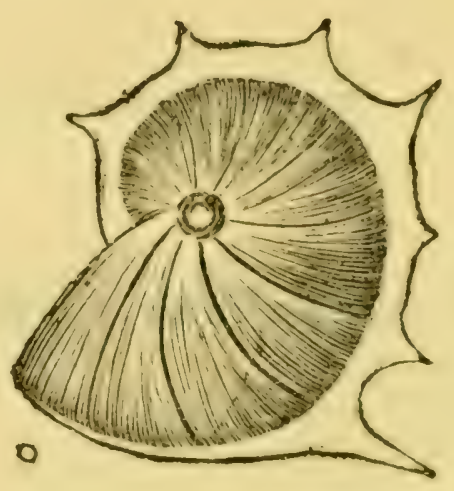

ANTÉNORE; cn lalin, Axtevor. 


\section{L'ANTÉNORE.}

Caractères génériques. Coquille libre, univalve, cloisomnée, en disque, et contournée en spirale; ayant un ombilic; le dernier tour de la spire renfermant tous les autres; bouche triangulaire, évasée, ouverte, recevant clans son milieu le retour de la spire; dos caréné et armé ; cloisons unies et percées par un siphon.

\section{Espèce servant de type au genre.}

\section{L'Anténore diaphane. Antenor diaphaneus.}

Cette coquille qui vient terminer la série de celles dont le dernier tour renferme tous les autres, et ayant un ombilic, vue ici au microscope, a, dans son état naturel et marin, une ligne de diamètre ; elle est diaphane, unie, et de couleur rosacée et irisćc; les cloisons se font remarquer au travers de sa transparence. On la trouve non-seulement dans les sables des rivages de lile de Bornéo, mais plus fréquemment sur les coralines qu'on arrache à une certaine profondeur. L'animal a huit bras, dont deux palmés, et il semble faire le milieu entre les poulpes testacés et les polypes; l'armure ou crête de la coquille semble formée de la glace la plus pure, et dans l'état de repos les bras palmés reposent sur elle. 
Simbinore diaphane est tres-rapproche de celui qur nous arons dejja dérit el grave dans notre hi-woire de's Molluspares, tome fr, parge ag, planche X.VH, ligure 5, sous la dínomination de nauile nicroscopingue de Solelani : ce savant aroit retire cette espice dn fomd ale la mer dans les environs de Lis onme et dr Porto-Fermajo (Soldani, test. vol. x. pas. 54. col. 1. tab. 55. F, 66. vas. 151.); et nous oloserverons ici 'pue les coquilles microsopiques sont repandues daus les ners des deux Inémisplicies, el rue de tres-fortes raisons font soupoumer qüil n'est aucme profondeur qui 'm soit exempte, et que leur multiplication y est prodigieuse.

Les anténores nous conduisent anx ammonies ou comies d'Ammon, qui n'en difièrent que paree que le dernier torm de la coquille n'enveloppe plus tous les antres, mais laisse au contraire entreroir la spire, en sicartant plus ou moins, et graduellement, du point central. 
UNIVAL VES.

Coquille is spire apparente. 
C OQUILLES

XIX: GENPE.

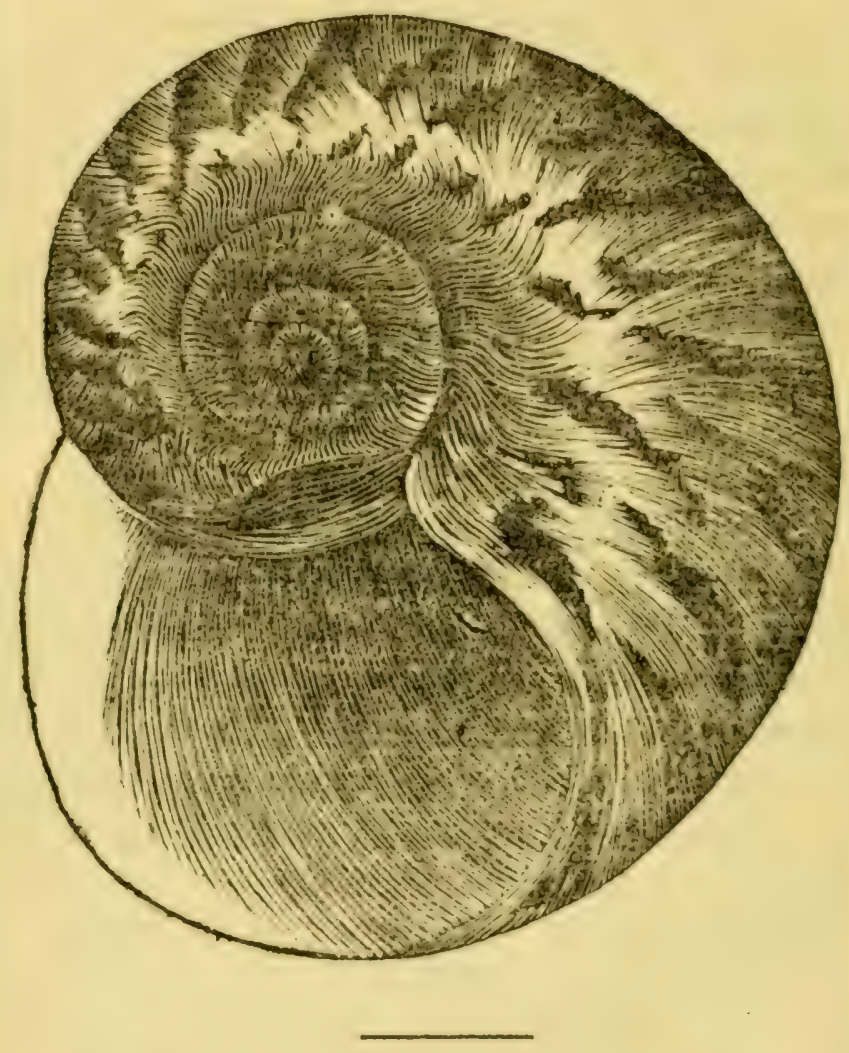

AMMTONIE; cn latin, Aruovites. 


\section{LAMMONIE.}

Caractères génériques. Coquille libre, univalve, cloisonnée, en disque, et contournée en spirale aplatie; tous les tours de spire apparens; bouche arrondie, évasée, recevant dans son milieu le retour de la spire; cloisons unies et toutes percées par un seul siphon.

\section{Espèce servant de type au genre.}

L'Ammonie flambé. Ammonites virgatus. Le grand nautile à spire de Favanne, et de plusieurs autres auteurs. En hollandois, denlevendigen ammons'hoorn.

Jusquà présent cette coquille rare a porté le nom de nautile : il est temps de lui assigner enfin sa véritable place. Elle ne peut point être rangée parmi les nautiles, parce qu'elle n'appartient pas à cegenre, et qu'elle en constitue un qui lui est propre. On ne peut donc être que trèsélonné lorsque l'on voit tous les auteurs qui cherchoient la corne d'Ammon à l'état marin, la méconnoitre dans l'ammonic dont nous parlons maintenant. Cette coquille faite pour orner les plus riches cabinets, a quelquefois neuf pouces dans son plus grand diamètre, sur un renflement de trois pouces et demi à son dernier tour de spire ; ses couleurs sont, à pou de chose près, celles du nautile flambé, ce qui a peut-être contribué à propager l'erreur et la cécité où on a été constamment à l'égard de l'ammonie flambé. A l'extérieur le têt estjaune, flambé de brun, l'intérieur est nacré, le retour de spire est chargé d'un enduit noirâtre semblable à celui du nautile, et qui prouve que l'ammonie est habité de même par une espéce de poulpe, qui jette aussi une liqueur noire dans certaines occasions. Cette belle coquille vient des mers de la Chine, et il est évident que c'est ici le type 
l'me espices d'ammonic: analogue dos ammonites ou comes d'Ammon a cloisons unies. Nous arons posscile: ume superbe pétrification le cet ammonic; elle aroit un pied dediamctre, et le dessin en est actuellement dans les porteteuillessle M. Fanjas de St. Fond; cet anmonitc cut verdiltre, argilo-pyrieux-calcaire, et vient des Vaches noires en Normandie.

Nous croyons deroir placer en ce lien, non pas la symonymie rles ammonies et ammonites, clle seroit fastidiense, mais, d'aprés notre plan, la nomenclature en plusicurs langurs. On tromye ders anmonites dans toutes les parties du monde.

Les ammonites se nomment en latin, ITammonila, cormu Hammonis, ammonia, oplyyociảa, oplyyomorphila, serpens hapideus, ceratoides, chrysolilha, ammonius lapis, ammoniles, ophyoüdes. En allemand, ammonilcn, ammonshorincr, widilerhormer, zicherharner, belschnectien, meenendret, scilfischslcine, drachensleine, schnectiensteine, sleinharrner, bergschnecticn, stcincrne schneckin, sclilingen. En francois, ammonite, corne d' Lmmon, cornedeluélier, fiento demoretre. En linllandois, ammonshoorn, ranshoorn, slangensiech, belslack, "ersteendeslang. Fin danois, ammonit, ammonshorm, sicculiorn, sinchliesicen, forstecnede slunger. En sucilois, ammoniter, ammonshorm. En anglois, lhc ramishom, the shacke slone, the sirpent slone. En inlien, ammonite, corno d'Ammoni, serpenti impetriti. In cspagnol, ammonila, cuerno de Ammon. In porlugais, ammonima, como de carnciro, corno de Hammon. En polonois, zaglick. 
Coquille en disque, ¿̀ spire apparente: 


\section{XX․ GENRE.}

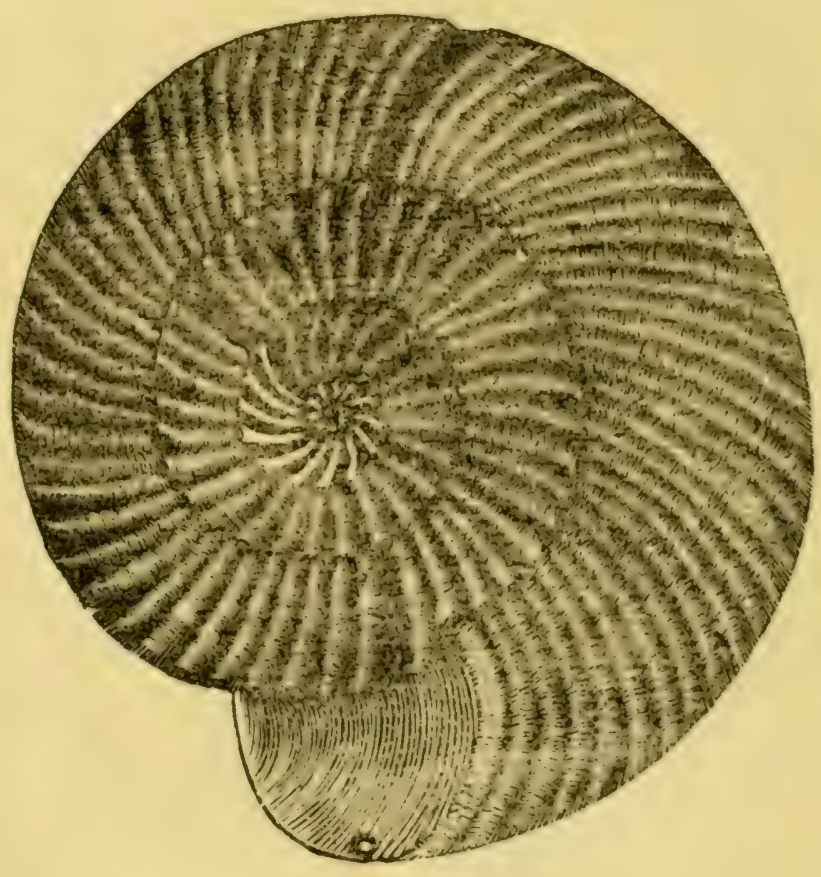

PLANUlite; en latin, Playulites. 


\section{LE PLANULITE.}

Caractères génériques. Coquille libre, univalve, cloisonnée, en disque, et contournée en spirale aplatie; tous les tours de spire apparens portant de distance en distance des étranglemens ou arrêts ; bouche arrondie, recevant dans son milieu le retour de la spire, cloisons unies, toutes percées par un seul trou.

\section{Espèce servant de type au genre.}

Le Planulite gauffré. Planulites undulatus. En belge, den gefronsden ammonshoren. Ammonites de six, sept, huit et neuf tours de spire de quelques auteurs.

On trouve des planulites dans presque tous les ouvrages qui ont traité des pétrifications; la synonymie en seroit immense, mais nous n'en connoissons pas l'analogue vivant ou à l'état marin. Les coquilles qui constituent ce genre sont en général beaucoup plus aplaties que les ammonies et les simplégades qui feront la matière du genre suivant: leurs tours de spire sont aussi plus nombreux, et on les trouve en aussi grande quantité que les simplégades, mais jamais nous n'en avons rencontré d'aussi énormes. Ses tours de spire sont coupés, de distance en distance, par de certains arrêts ou étranglemens où l'ondulation ou gauffrure est interceptée : ils offrent une espèce de repos, indication probable de l'âge de l'animal; dans tous les cas il est évident que ces ressauts 
sont les indices dim ritat momentime de repos on dinaction chez le nollusque constructeur de ces corpuilles, qui, retenant en contraction quelifues-mes de ses partics, ne lui permit pas de former à ces époques les bourrelets ou ondes qui caractérisent l'espice qui nous sert ici de type: ces intervalles sont aussi un des caractires du genre.

Les planulites se rencontrent partout à la surface du globe, milés avec d'autres pétrilications pedagriennes; ils y sont dans tous les élats communs aux autres fossiles; il en est d'agathisés, comme de calcaires; de pyriteux et de convertis en mine de fer, soit hímatite, soit ocracéc: on les trouve dans les plaines comme sur le sommet des montagnes. Juscu'à ce jour, ils n'avoient porté d'autre dénomination que celle de corne d'Ammon, ct le lecteur peut juger jusqüà quel point ils en different. 


\section{Coquille en disque, à spire apparente.}




\section{GENRE。}

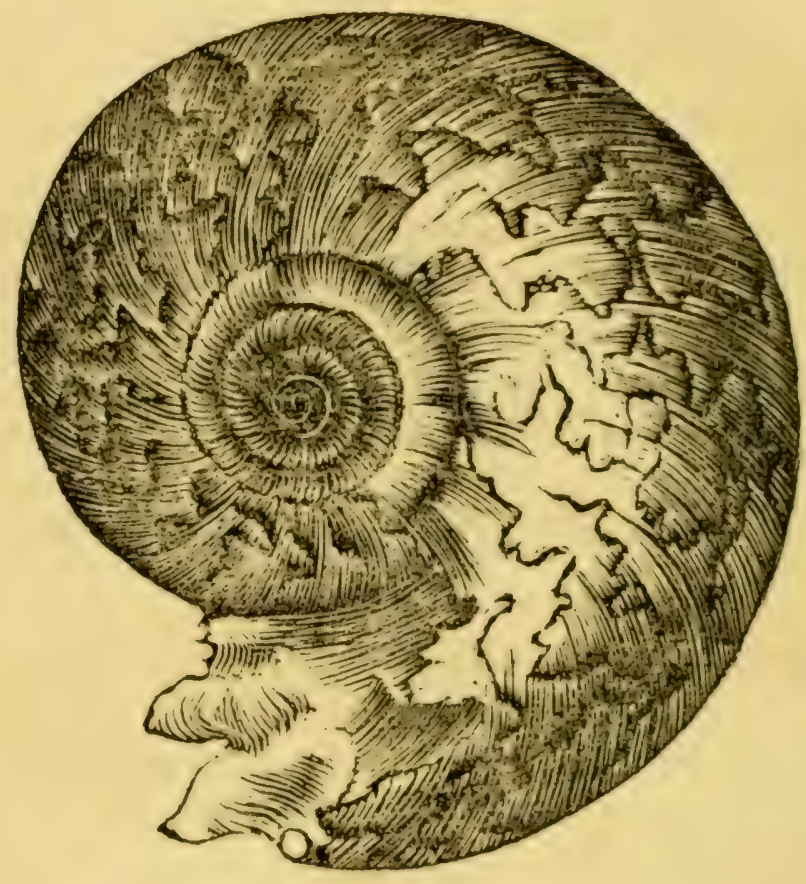

SIMPLÉGADE; en latin, Simplegades 


\section{LE SIMPLÉGADE.}

Caractères génériques. Coquille libre, univalve, cloisonnée, en disque, et contournée en spirale aplatie; tous les tours de spire apparens; bouche arrondie, recevant dans son milieu le retour de la spire; cloisons dentelées, lobẻes, persillées, et percées par un seuI trou.

\section{Espèce servant de type au genre.}

Simplégade couleuvrée. Simplegades colubratus.

Dans ce genre nous ne connoissons encore rien à l'état vivant ou marin; et les simplégades confondus sous la dénomination banale de cornes d'Ammon, portoientpour épithète caractéristique celle de persillées. On ne les connoit qu'à l'état fossile, ou ils remplissent tous les modes de pétrifications : on les a rencontrés dans toutes les couches calcaires du globe, depuis la grandeur d'une lentille jusqu'à celle de huit pieds de diamètre, et même davantage; volume vraiment énorme, et qu'on auroit peine à vouloir admettre s'il n'en existoit même à Paris, dans le Musée impérial d'histoire naturelle, d'environ quatre pieds. Les simplégades sont souvent ferrugineuses, et d'autres fois encore elles ont conservé une partie de leur nacre et de leur orient : à l'état pyriteux, elles paroissent bronzées, et c'est ce que les 
Alkmands nomment cones d'Anmon habillées, armies (geharnischte Ammonshorn ). Il est très-probable que les simplégades, comme beancoup d'antres mollusques pélagiens, vivent daus le fond des hautes mers, el qu'une cause physique quelconque ne leur permet point de paroitre à la surface des eaux : ricn au contraire n'est aussi rarié que les nombreuses espéces de simplierades que l'on rencontre dans lesflancs entr'ouverts des montagnes, dont la formation est évidemment due aux catux d'un antique océan, déplacé par quelque grand calaclysme, dont les traces existent bien pour nous, mais :lont l'époque se perd dans l'impénétrable nuit des sierles. C'est-là que les simplégades, les ammonites, les flanulites, les nautilites gisent au milieu d'une immensité d'autres coquilles fossiles, restes, comme cux, et débris de générations antiques dont les dépouilles ont survécu aux âges; médailles probantes de la série des siecles que la main du temps a accumulés sur le globe. 
Coquille en disque, à spire apparente. 


\section{XXIIe。GENRE.}

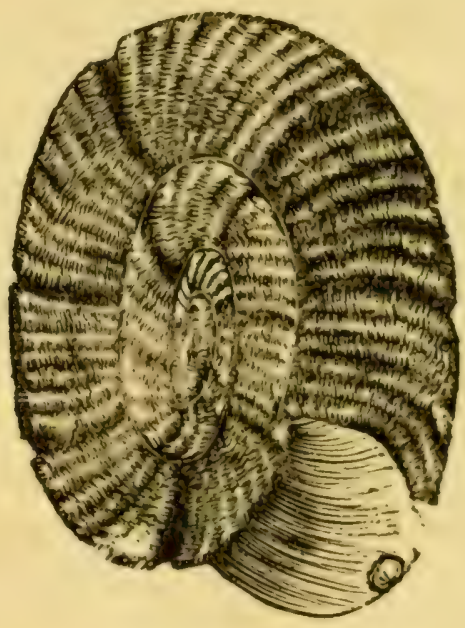

ELLIPSOLITE; en latin, Elt.ipsonithres. 


\section{L'ELLIPSOLITE.}

Caractères génériques. Coquille libre, univalve, cloisonnée en ellipse, et contournée en spirale; tous les tours de spire apparens; bouche arrondie, recevant dans son milieu le retour de la spire; cloisons unies, percées par un seul trou.

\section{Espèce servant de type au genre.}

Ellipsolite cordonné. Ellipsolithes funatus. Corne d'Ammon elliptique.

Quoique singuliers par leur conformation, et assez rares, les ellipsolites comptent cependant sept à huit espèces qui viennent se ranger sous le genre qui leur est propre. Lindividu que nous décrivons vient de la montagne de Sainte-Catherine, près de Rouen; nous l'y avons rencontré aggloméré avec quelques turrilites, et d'autres pétrifications de ce genre ; il est de couleur grise jaunissante, et sa matière est argilo-calcaire; on voit que ses tours de spire portent, de distance en distance, des étranglemens ou arrêts plus rapprochés que ceux qui caractérisent les planulites, et qui paroissent résulter de la même cause. La forme elliptique est constante dans ces fossiles, et elle n'y est due à aucun accident qui auroit écrasé ou déprimé la coquille, soit pendant la vie de l'animal, soit à toute autre époque. Nous 
avous vus souvort, "t dans plusienrs rabinels, des fragmens rillipsolites quion mous présentoit comme des

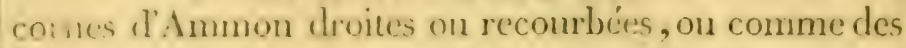
linites; mais lerreur ust farile à reconnoitre, en ce que jamais ces fragmens ne sont terminés, que les lituites le sont par leur sommet, et que les baculites ou cornes d'Ammon droites ne dévient point de cette ligne; d'ailleurs, pour des regards excrerir, cette erreur ne perut avoir lieu, et il est facile de distinguer au premier coup-d'ocil le fragment mème d'un lituite d'avec celui des ellipsolites.

Nons avons dessiné celui-ci de grandem naturelle; mais il y en' a de plus grands.

L'analogue à l'état virant n'est point connu. 
Coquille en disque, à spire apparente. 


\section{GENRE.}

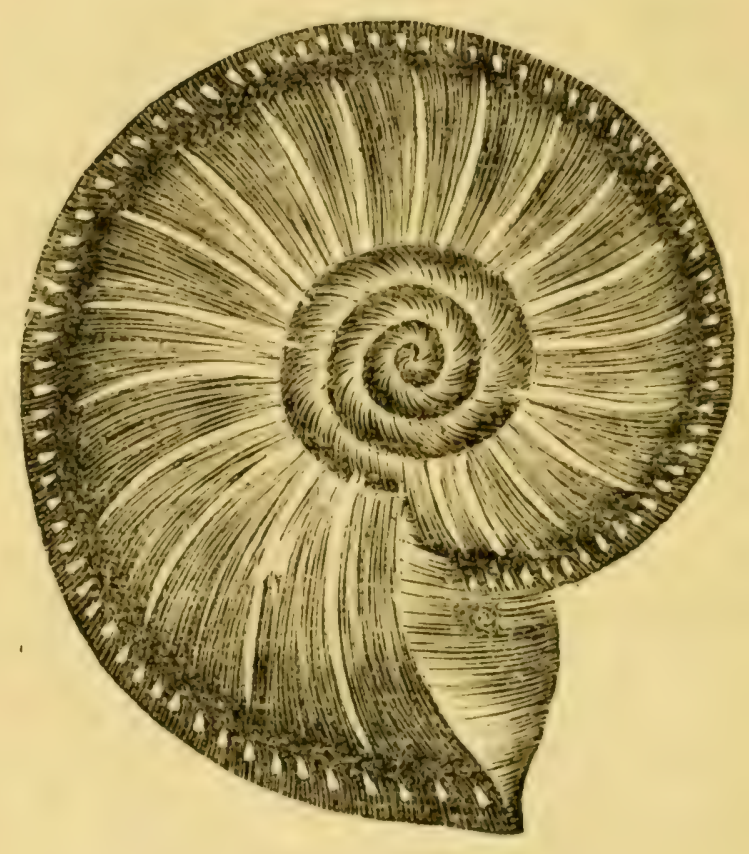

AMALTÉ; en latin, Amaltueus. 


\section{L’A M ALTÉ.}

Caractères génériques. Coquille libre, univalve, cloisonnée, en disque, et contournée en spirale aplatie ; tous les tours de spire apparens; dos caréné et armé, bouche triangulaire, recevant dans son milieu le retour de la spire; cloisons unies, percées par un seul siphon.

\section{Espèce servant de type au genre.}

Amalté perlé. Amaltheus margaritatus. Corne d'Ammon à dos caréné.

L'amalté est encore plus aplati que les planulites et les ellipsolites; beaucoup de coquilles pétrifiées de ce genre ont encore conservé leur nacre et leur orient, d'autres sont ocracées et ferrugineuses. On en trouve qui ont plus de cinq pouces de diamètre. Le dos des amaltés, au lieu d'être arrondi, est caréné et armé d'une crête, ce qui rend leur bouche triangulaire lancéolée. Celui dont nous donnons la figure est encore nacré et vient des environs d'Anvers. Ce genre est assez nombreux en espèces fossiles, qu'on retrouve dans tous les cabinets, mais nous n'en connoissons pas encore l'analogue vivant, et sous ce rapport nous sommes forcés de ranger l'amalté parmi les coquilles pélagiennes, que quelqu'heureux hasard nous pourra faire connoitre un jour. 
Indépendammont des coquilles entieres, on rencontre souvent des fragmens d'amalté: il en est de móme des planulites, desammonites, des simplégades; souvent il n'en reste que cing à six articulations, et comme elles sont plus ou moins arrondies, on a cru y reconnoitre res queues ou corps d'écrevisses, et en conséquence on leur a douné le nom de Caudes cancri. C'est une erreur, non pas qu'il n'y ait des écrevisses pétrifiées, auxipuclles les oryctologues ont donné la dénomination d'astocolithes, mais clles sont beaucoup plus rares, et recounoissables sous une foule de rapports. Il en est de même, lorsque par suite des temps ces coquilles fossiles ont perdu leurs cloisons; alors la matiere solide qui remplissoit leurs concamérations se trouve isolée, et chaque articulation libre joue avec celles qui lenr sont voisines; ces accidens sont fréquens, et bien connus par les naturalistes qui se plaisent ì étudier les fossiles, à les réunir et à les considérer souvent. 
Coquille en disque, à spire apparente. 


\section{XXIV'. GENRE.}

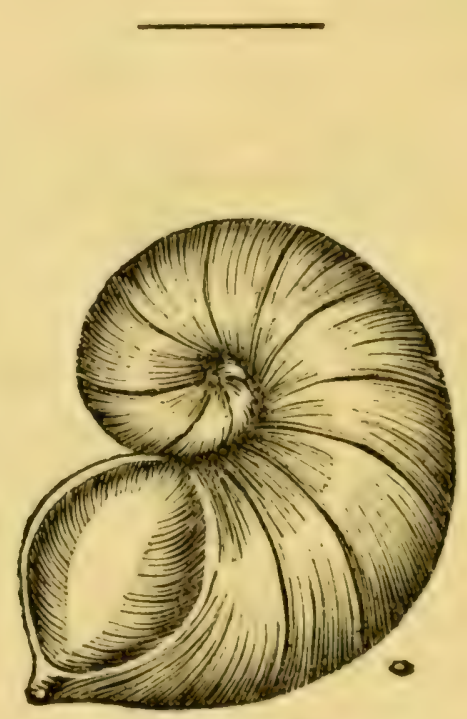

ORÉADE; en latin, Oneas. 


\section{L'ORÉ ADE.}

Caractères génériques. Coquille hibre, univalve, cloisonnée, en disque, et contournée en spirale; tours de spire apparens, adhérens, mais sans enchâssement ; bouche piriforme, scellée par un diaphragme bombé, mamelonné à l'extrémité extérieure ou il est percé par un petit siphon; cloisons unies. Le retour de la spire hors de la bouche.

Espèce servant de type au genre.

Oréade cornet. Oreas subulatus. - Nautilus acutauricularis. Der spitzohrschiffer. Testac. microsc. a Leo. von Fichtel et J.P.C. von Moll, pag. 102. tab. 18 , fig. $g$, h, i. - Soldani, testac. T. I. P. x. pag. 6r. tab. 49 , fig. $x$ ?

Cette coquille assez évasée, paroît tenir le milieu entre les ammonies, les genres qui viennent s'y rattacher, et les spirules; ellen'a point une spire circonvolutée comme les premiers, et dans le peu qu'elle en présente, les tours sont adhérens, au lieu que ceux des spirules sont détachés. L'Oréade en seroit cependant très-rapproché, si sa bouche scellée ne l'en éloignoit pas entièrement. Fondés sur ces caractères, nous avons cru pouvoir en faire un genre particulier, et pour nous il termine la série des cornes d'Ammon proprement dites, que nous nous flattons d'avoir enfin mises en ordre, en les divisant simplement en cinq genres, ou toutes viennent 
Se ranger. C'est le travail yui nous a coulé le julus de recherches, et cest par suite des nombreux et volumineux onviages que nous arons consulés, que noms

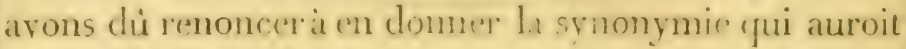
occupré a clle sente des peges tout entieres: d'antant plus que nous sommes con aincus glume bonne ligure raut it elle seule toutes les synonymies.

La conleur de l'oréade cornet est hlanche, teintée de bleu et de violet; on la trouve parmi les concrétions marines, et engagée dans les coralines de la Méiliterrannée; clle a quelquefois une demi-ligne de diamitre.

En terminant ce rqui concerne les nautiles et les cornes d'Ammon, il nous reste encore une obscrvation à faire, et qui appartient à l'étude des fossiles; elle se rapporte aux empreintes et creux qui se détachent du redief de ces pétrifications; rénérés par les Indiens, ils en ont recu le nom de Salagramman; quelques naturalistes atlemands les nommèrent Naulilus ammonites sacer. Les oryctologues leur domerent la dénomination d'ammonitot)politluc, ou uspe pétrifié d'ammonite. Voyez surtout Blumenbach, Specinen archeologice tclluris. Goettingue, 1805 , planche 11 , fig. 7 . 
Coquille à spire en disque, et contournée en trompe. 
$9^{8}$ COQUILES

XXVe. GENRE。

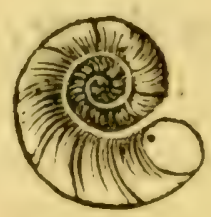

SPIRULE; en latin, Sinuld。 


\section{LE SPIRULE.}

Caracteres génériques. Coquille libre, univalve, cloisonnée, en disque, et contournée en trompe aplatie; tous les tours de spire séparés; bouche ronde; cloisons unies, et toutes percées par un siphon conlinu et placé contre le bord intérieur de la coquille.

\section{Espece servant de type au genre.}

Spirule fragile, Spirula fragilis. Lamarck, Syst. des anim. sans vert. pag. I o2. F. de Roissy, Hist. nat. des Moll. Buffon de Sonn. tom. 5, pag. 15, pl. ı6, fig. I. Nautilus spirula.Linn. Cornu Anmonis spurium. Le cornet de postillon. D'Argenv. pl. V. fig. G. Rumph. mus. tab. 20. f. r. Martini, conch. I. tab. 20. f. I 84. I 85. En allemand, das unæchte Ammonshorn, Widderhorn, Posthorn, Mewendrek. En hollandois, Posthoorntje. En belge, Posthooren. En anglois, the Ramshorn-Sailor. En françois, Cornet de St.-Hubert, cornet I chambré, fiente de mouette. En malais, Taymanu-samal.

Les conchyliologues modernes ont confondu en général cette jolie coquille avec les lituites; mais les lituites, indépendamment du commencement de leur spire, faite en trompe, prolongent cette spire à une certaine époque, et la jettent en avant, droit et sans lui donner aucun enroulement; ce qui nous a décidé, d'accord avec les faits, à en former un genre particulier; le spirule, au contraire, reste toujours roulé en trompe, 
nous en avons vu des millions, et jamais nous n'en avons rencontré un seul qui se soit écarté de cette forme. Dëjà depris plus de dixans nous en connoissions l'animal, que nous nous étions proposé de publier it son tour dans l'histoire des mollusques : 1MM. de Roissy et Péron nous ont prévenu; cclui-ci, naturaliste du gouvernement dans la dernière expédition autour du monde, a rapporté, au milieu d'une foule d'autres objets du plıs grand intérêt, une coquille renfermant cncore son individu, quil a trouvé mort et flottant sur les eaux, a mme grande distance des côtes. Rapproché, d'un côté, des poulpes constructeurs d'un têt ou coquille, le spirule a une tète entourée de huit bras courts et de deux autres plus longs; et voisin, d'un autre côté, des calmars, les deux côtés qui terminent son corps un peu allongé, couvrent, comme le feroit un manteau, toute sa coquille, qui d'apres cette raison est toujours lisse et nacrée. Le spirule est un mollusque pélagien, il flotte et vogue en troupes dans les hautes mers, et ne rient point visiter les rivages; il est ichthy ophage, car nous lui avons recoimu un bec comme celui des séches, des poulpes et des calmars. Chez lui les sexes sont séparés, il en est de mâles et de femelles. Les spirules vivent dans les mers de l'Amérique et de l'Inde, et après les tempétes on trouve souvent une quantité de leurs coquilles jetées sur la côte dans les íles indiennes, à Amboine, à Bornéo surtout, mais elles sont vides, parce que le mollusque ne tient à sa coquille que par un petit filet charnu trés-aisé à rompre. La coquille est nacrée, presque diaphane, teintée des sept couleurs prismatiques, et perlée ; elle atteint quelquefois jusquà un pouce de diamètre. 
UNIVALVES.

Coquille à spire en disque, et contournée en trompe. 
XXVI. GENRE.

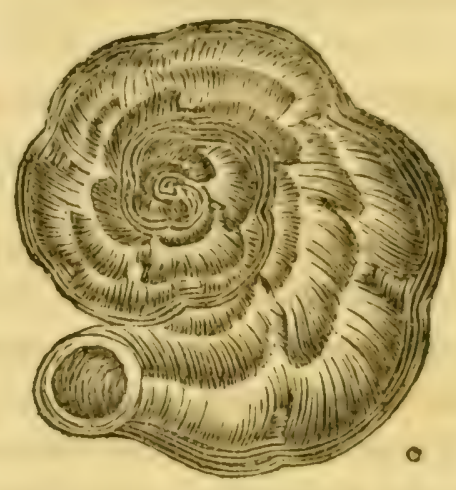

JESITE ; en latin, Jesites. 


\section{LE JÉSITE。}

Caracteres génériques. Coquille non-libre ou adhérente à d'autres corps, univalve, cloisonnée, en disque, et roulée en trompe; tours de spire adhérens; bouche écartée du corps de la coquille, ronde et plus petite que lintérieur de la dernière concamération; dos à demicaréné; cloisons unies; siphon inconnu.

\section{Espèce servant de type au genre.}

Le Jésite vermicule', Jesites vermicularis. Polythalame de Soldani, Testac. tab. 3o. vas I 43. X.

Plus nous avançons dans te champ des coquilles microscopiques, et plus nous trouvons à glaner, indépendamment de nos propres observations. Actuellement nous avons devant nous une corne d'Ammon, ou espèce d'ammonie, d'après tous les nomenclateurs qui nous ont précédés, mais formant un genre particulier en raison de sa bouche, ronde, singulière, étranglée, et qui se projette en avant, passage qui pourroit nous conduire, s'il n'existoit d'autres intermédiaires, des spirules aux lituites. Cette bouche est plus petite que la chambre ou camération qu'elle termine, de façon qu'elle forme une espèce de col ou de gorge étranglée par un bourrelet ou cordon. Du reste la coquille est arrondie, le dos en est à demi-caréné, et on voit distinctement tous les tours de spire, comme dans les ammonies proprement dites, 
mais ici la boucle ne recuit point le retour de la spire dans son intérieur.

Le Jésite vermiculé a cncore un autre caractère distinctif ou spécifique : son têt, indépendamment des stries ordinaires d'accroissement, porte aussi des espèces d'enupreintes qui lui donnent un aspect mâché; les cloisons sont peu apparentes à l'extéricur, mais cependant assez indiquées pour montrer qu'elles sont éloignées les unes des autres.

Soldani est le premier et le seul jusqu’à présent qui ait figuré et décrit cette espèce, elle a une ligne de diametre dans sa plus grande dimension; sa coquille, quoique assez solide et non-diaphane, est teintée de rose; on la trouve cn grande quantité sur les algues, les coraux, les crusLacés el les coralines de la Méditerranée; elle s'y attache isolément, et paroît parasite. 
Coquille à spire en cisque, sommet écrasé. 
XXYII. GENRE。

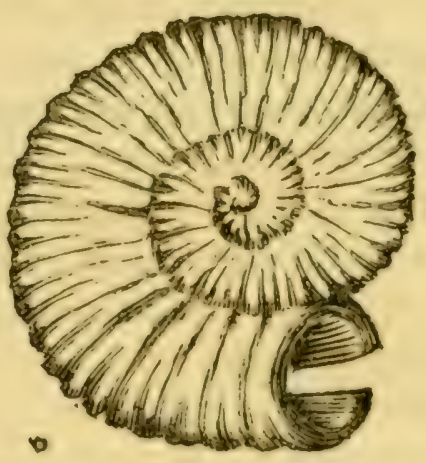

CHatribDE; en latin, Cratums 


\section{LE CHARIBDE.}

Caractères génériques. Coquille non-libre, univalve, cloisonnée, sommet en spire écrasée, et base aplatie ; bouche ronde; entaillée par-dessous; cloisons unies, siphon inconnu.

\section{Espèce servant de type au genre.}

\section{Le Charybde froncé, Charybs plicatus.}

Polythalame de Soldani, testac. tab. 29. vas. I43. k. et pag. 33.

Cette coquille qu'on trouve, de même que la précédente, sur l'algue, les plantes marines, sur le têt des crustacés, et dans les ramifications des coralines de la mer Méditerranée, présente une bouche échancrée par une solution de continuité; ainsi que nous le verrons dans celles des pleurotomes, lorsque, dans le second volume, nous publierons les coquilles univalves noncloisonnées : cette fissure ou échancrure se trouve encore de même dans quelques lepas ou patelles en cabochon, dont les conchyliologues ont fait maintenant des genres particuliers. Nous avons donc ici une preuve nouvelle que la nature remplit toutes les places, tous les interstices, et qu'il seroit même difficile à l'imagination la plus vive d'enfanter des formes dont elle n'offre les types, en les répétant même sous une foule de modifications ; idée qui naît de la vue des faits, et qui se confirmera de plus en plus, à mesure de l'accroissement de la masse de nos connoissances. 
Les Charybdes sont contournés i-peu-près dans le genre des cornes d'Ammon; mais ces coquilles ne sont plus libres, elles sont adhérentes, ce qui les en sépare, indépendamment de da scission de leurs bouches. Leur spire rentrante de même d'un côté, est aplatie sur un seul plan de l'autre, en raison du corps sur lequel elles s'attachent et croissent. Elles s'isolent les unes des autres, et cependant paroissent vivre en famille, adhérentes, comme les corquilles du genre qui précède. On peut en inférer que les mollusques qui les construisent ont une autre organisation que celle des séches et des poulpes, et que plus rapprochés des poulpes à bras, ils réunissent les deux sexes, ct sont par conséquent androgynes, à moins que le mâle ne jette un frai, une aure fécondante pour vivifier les œufs de la femelle. Le charybde froncé a sa coquille toute plissée, et comme boudinée ; tous ces bourrelets sont finement striés dans le sens du prolongement de la coquille, qui a quelquefois une ligne de diamètre. Elle est blanche, teintée de vert et de rose, et elle est très-abondante dans toute la Méditerranée. 
Coquille à spire en disque, sommet éminent. 

I 10
COQUILLES

XXVIII. GENRE.

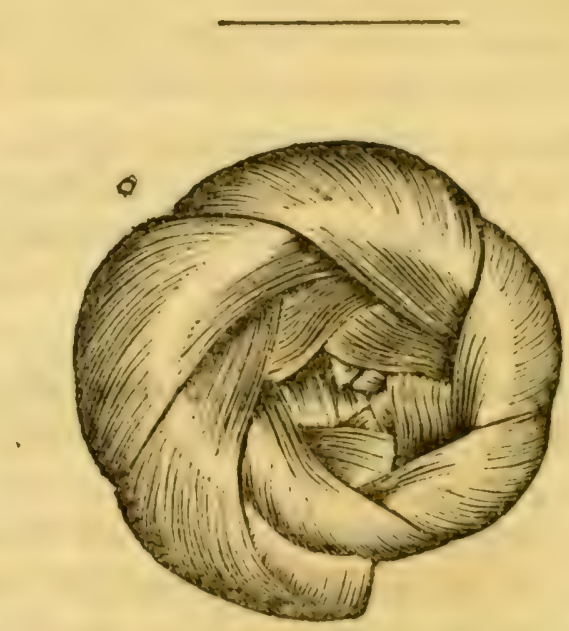

CiDArOLle; en latin, Cinarolius. 


\section{LE CIDAROLLE.}

Caractères génériques. Coquille libre, univalve, cloisonnée, en disque, à spire éminente et base aplatie; roulée en forme de turban; bouche ouverte, recevant verticalement le retour de la spire; cloisons unies; siphon inconnu.

\section{Espèce servant de type au genre.}

Cidarolle étoffé; Cidarollusplicatus.

Polythalame de Soldani, testac. tab. 36. vas. 160. S.

Ce genre est d'autant plus remarquable qu'il nous présente un singulier enchevêtrement des concamérations de la coquille, qu'on ne peut, à ce qu'il nous semble, mieux comparer qu'aux plis roulés d'un turban. Le cidarolle étoffé est parfaitement arrondi, le sommet est obtus, mais aplati à la base. Il n'y offre cependant point encore d'ombilic; cette configuration nous mène insensiblement aux genres qui vont suivre. Les cloisons sont assez espacées les unes des autres, mais elles dessinent les concamérations en forme de sacs presque triangulaires et renflés, et il est probable que l'animal du cidarolle se rapproche infiniment de celui qui habite les spirules, d'autant plus que le genre que nous décrivons n'offre que des coquilles libres, et nonadhérentes. 
Comme presque toutes les coquilles microscopiques, le cidarolle étoflé est diaphane et perlucide; il reflette d'ailleurs les coulcurs de l'iris, et il se fait encore remarquer par le brillant d'une nacre extrêmement fine. Tout porte à croire qu'il multiplic extrêmement, car on le trouve en très-grande quantité dans les sables des plages, aux environs de Livourne, ainsi que sur les rivages de la Toscane.

Sa grandeur est de deux tiers de ligne. 
Coquille spirée, sommet éminent:

$$
\text { I. }
$$




\section{XXIX'.GENRE。}

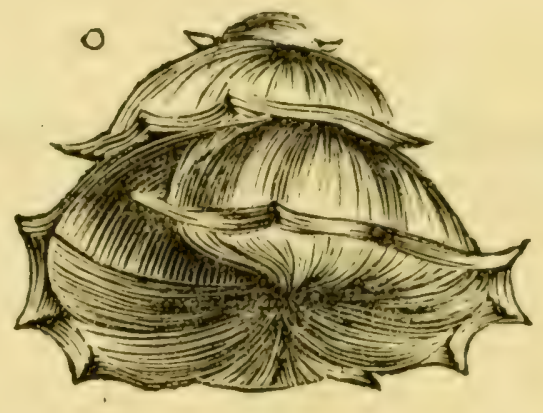

CorTale; en latin, Contalus. 


\section{LE CORTALE.}

Caractères génériques. Coquille libre, univalve, cloisonnée, à spire saillante, élevée en sommet, et base aplatie ; bouche triangulaire, ouverte, recevant verticalement le retour de la spire; dos caréné et armé; cloisons unies.

\section{Espèce servant de type au genre.}

Le Cortale pagode, Cortalus pagodus.

Polythalame de Soldani, testac. tab. 86. vas. 162. X.

Cette coquille cloisonnée est la première qui, dans notre système ou arrangement classique, nous présente une spire assez relevée, assez distincte pour nous permettre d'y compter deux tours; cependant ces retours de spire, quoique verticaux, se logent encore par leur retour successivement dans la bouche, à la manière des cornes d'Ammon, et d'après cette position nouvelle, le dos devroit porter ici le nom de ventre, comme il le fait dans les toupies et dans les sabots avec lesquels cette coquille auroit de grands rapports d'analogie , si elle n'étoit pas chambrée ou cloisonnée. A l'extérieur elle en a la forme, et c'est ce nouveau rapprochement qui nous a forcés à faire un ordre entier des coquilles cloisonnées, ordre dans lequel on retrouve toutes les formes, qui à l'extérieur caractérisent toutes les coquilles non-cloisonnées, au point que l'on peut retrouver toutes ces formes extérieures dans les deux 
ordres. Ce ventre ou dos est armé ou caréné dans les cortales, ce qui rend leur bouche triangulaire; la carine est courte, ćpaisse, et armée de fortes saillies épineuses, et dessinées en festons renversés.

La coquille du cortale pagode est diaphane, et on voit distinctement au travers du tèt les cloisons assez Ćcartées les unes des autres, elles sont unies; la base de la coquille est aplatie, un peu renfoncéc au centre, mais sans ombilic.

Elle est nacrée et teintée des couleurs de l'iris, le fond est cependant perlé.

Elle a une ligne et demi de diamètre; et on la trouve dans la Méditerranée, mais principalement sur les rivages de Livourne.

Cette coquille nous mène à celles qui, indépendamment de leurs cloisons, sont turbinées. 
Coquille spirée, turbinée. 


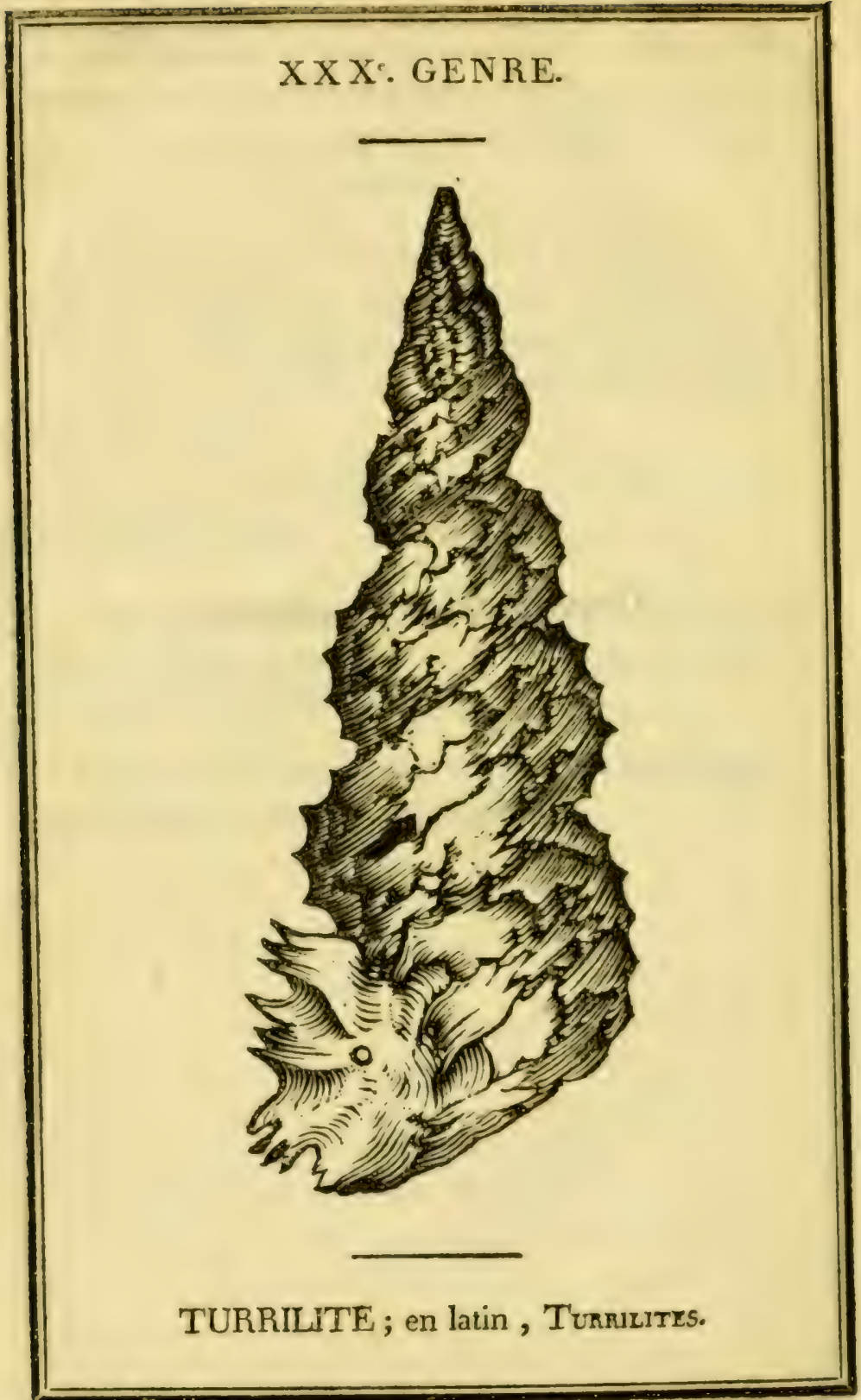




\section{LE TURRILITE.}

Caractères génériques. Coquille libre, univalve, cloisonnée, contournée en spire turbinée et élevée, les tours de la spire contigus et apparens; bouche arrondie; cloisons dentelées et persillées, percées au centre par un seul trou ou siphon.

\section{Espèce servant de type au genre.}

Turrilite turbiné, turrilites costatus; corne d'Ammon turbinée. Montfort, Monogr . Journ. de phys. therm. an 7 , p. I, t. I, f. I. Lamarck, Syst. des an. sans vert., pag. 102. F. de Roissy, Hist. des moll. Buff. de Sonnini, tom. V. pag. 30.

Nousfùmes les premiers à faire parfaitement connoître ces coquilles si remarquables, et qui forment la nuance intermédiaire principale entre les cornes d'Ammon, les nautiles, ou coquilles à spire aplatie en disque, et celles dont la spire est relevée et turbinée. Nous en avions reconnu plusieurs espèces, et nous avons vu que tous les nomenclateurs qui écrivirent après nous sur les coquilles, ont adopté ce genre dans toute son intégrité, et tel que nous le représentons aujourd'hui. Cependant quoiquil y en ait plusieurs espèces, on n’a encore rencontré les turrilites quà l'état fossile: Langius, Bayer, Scheuchzer, Bourguet en avoient publié quelques fragmens informes, mais en ayant rencontré d'un pied et demi de long dans nos courses lithologiques, nous publiâmes nos observations, accompagnées d'une bonne 
ligure, dans le journal de plysique. Dëjà on en connoit (fuatre espices, el nous pourrons un jour en publier davantage. Cette coquille singuliere peut encore nous conduire aux baculites, aux hippurites, et a tous les genres nombreux de coquilles cloisonnées droites ou peu arquées et contournées, qui feront partie de ce volume.

Quoiqu'entièrenent pétrifiées nous en avons trouvi qui avoient conservé leurs têts; il est mince, blanc, solide, peu nacré; mais fon sent combien l'état fossile a dul'altérer. En général, nous avons trouvé les turrilites dans les bancs de matiere calcaire crayeuse des environs de Rouen; d'autres d'une couleur grisâtre et argileuse, dans les environs du Havre, et dans ces deux cas, les concamérations sont remplies d'une matière lomogine ou semblable à celle qui constitue les bancs ou ils sont ensevelis; trés-souvent les articulations se délachont les unes des autres, et alors les persillures se dessinent avec la plus grande netteté. Nous avons possédé des fragmens de turritiles entierenıent agathisés, mais ce mode de pétrification est trés-rare. Tout prouve que ces coquilles furent pélagiennes, et que les animaux qui les construisirent étoient très-rapprochés de ceux des nautiles et des spirules. 
Coquille à spire raulée, sommet en pain de sucre. 


\section{GENRE.}
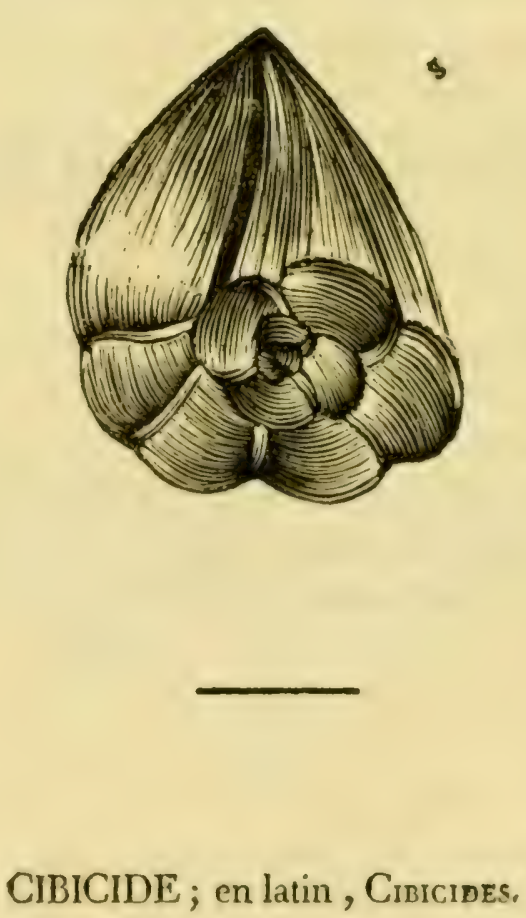


\section{LE CIBICIDE.}

Caractères génériques. Coquille libre, univalve, cloisonnée, à base aplatie, le sommet conique, élevé en pain de sucre; bouche linéale, de toute la hauteur de la coquille, et appuyée contre le dos, ou retour de la spire; cloisons unies.

\section{Espèce servant de type au genre.}

Cibicide glacé, Cibicides refülgens.

Polythalame de Soldani. Testac. tab. 46. vas. 170.

Nous venons de voir une coquille cloisonnée avec une spire véritable et apparente, dont chaque tour renflé s'appuie et se soude aux deux tours qui lui sont voisins: ici c'est toute une autre modification. Quoique le cibicide présente un sommet élevé, ce sommet n'est plus le point de l'origine de la coquille, et il est au contraire l'ouvrage des concamérations successives qui s'enroulent sur les premières, et les recouvrent, parce que successivement elles grandissent avec l'animal qu'elles renferment, de façon que les premiers tours de cet enroulement sont renfermés dans le centre de la coquille; cependant comme la base est aplatie, la spire ou l'enroulement tout entier s'y dessinent, et l'œil peut aisément suivre ses progrès:les concamérations occupent toute la longueur, depuis le sommet jusquà la base, de manière à former une section de cône; cha- 
cune d'elles se bombe dans son milieu, et est fermée hermétiquement partout ailleurs quà l'endroit de la bouche. On ne sauroit mieux la comparer quà une côte d'orange qu'on auroit coupée en deux : cette bouche qui régne tout le long de la concamération est linceale, c'est-à-dire que non-seulement elle est en longueur, mais encore qu'clle n'a que tres-peu d'ouverture, représentant beaucoup plutôt une fente qu'une bouche; elle est appuyée sur le retour de la spire.

Le cibicide glacé est diaphane, nacré ct irisé : on le rencontre à l'état marin, comme à celui fossile, prés de Jivourne en Toscane, et dans le territoire de Sienme; dans l'état fossile sa couleur est tantôt ferrugineuse, et tantôt ardoisće, en raison des bancs ou lits dans lesquels on le rencontre. Sa grandeur est celle d'un point géométrique. 
Coquillè̀ spire, roulée, globuleuse. 


\section{XXXII'。 GNRE.}

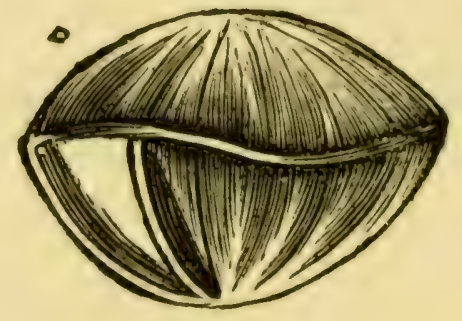

EPONIDE; en latin, Eponides. 


\section{L'ÉPONIDE.}

Caractères génériques. Coquille libre, univalve, cloisonnée, spirée et globuleuse, ou presque lenticulaire, le têt recouvrant la spire; sommet et base formés en calotte; marge carénée; bouche située à la base, dessinée en arc, et ayant en longueur le quart de toute la circonférence, triangulaire, partant du centre de la base, et se rendant à la carène ; recouverte par un diaphragme ouvert contre le retour de la spire, et offrant à son angle extérieur un siphon; cloisons unies.

Espèce servant de type au genre.

L'Eponide déprimé, Eponides repandus.

Nautilus repandus, der ausgeschweifte schiffer. Testac. microsc. a Leo. von Fichtel et J.P. C. von Moll, pag. 55. tab. 3. f. a , b , c, d.

D'après la longueur de la phrase caractéristique, nos lecteurs jugeront combien cette coquille differe de toutes les autres qui ne lui sont point congénères; elle n'est ni un nautile, ni une camérine, et forme un genre absolument à part par la singularié de sa construction, et particulièrement par celle de sa bouche. La spire est totalement masquée et recouverte par le têt, et les premiers tours, ceux de l'origine, sont au centre; tout porte à croire que l'univalve de l'éponide est pourvu d'un 
manteau, au moyen duquel il enveloppe sa demeure; le siphon indique encore un appendice ou fil charnu qui l'attacheroit à elle, comme l'est l'animal du nautile à la sienne.

Le mollusque de l'éponide se nourrit sur les algues et sur les plantes marines de la Méditerranée, il y est assez rare: la couleur de sa coquille est d'un jaune blanchâtre sur lequel se dessinent les cloisons, traversées par la carène, en jaune beaucoup plus foncé; elle est légèrement nacrée, et a une demi-ligne de diamétre.

On peut regarder l'éponide comme formé par deux hémisphères ou caloltes qui seroient réunis par une carène circulaire. 
Coquille à spire élevée, base aplatie. 


\section{XXXII". GENRE.}

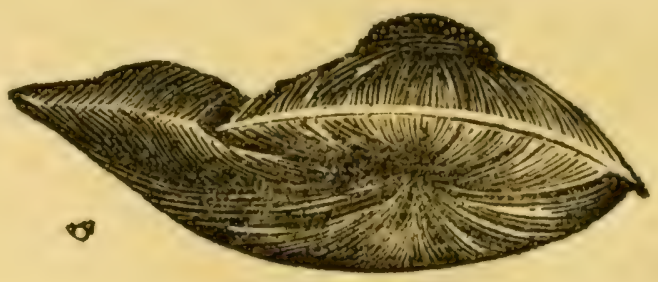

STORILLE; cn latin, Storilus. 


\section{LE STORILLE.}

Caractères génériques. Coquille libre, univalve, cloisonnée, à spire saillante, couronnée par un mamelon , base aplatie, dos caréné , bouche lancéolée, recouverte par un diaphragme, ayant une ouverture contre le retour de la spire qu'elle reçoit dans son milieu; cloisons unies; siphon inconnu.

\section{Espèce servant de type au genre.}

\section{Storille rayonnant. Storilus radiatus.}

Cette jolie coquille differe de toutes celles des autres genres en ce qu'elle est surmontée d'un mamelon qui indique le sommet d'une spire, dont les tours se dessinent d'ailleurs avec l'âge. Dans l'espèce que nous décrivons, ce mamelon est percé d'une infinité de petits trous, il en est, pour ainsi dire, criblé; la base estaplatie, et laisse soupconner un ombilic central : l'aspect général de la coquille se rapproche de celui du sabot, et c'est ainsi que successivement nous voyons toutes les formes des coquilles non-cloisonnées, venir se répéter parmi celles qui le sont ; tandis que d'un autre côté ces coquilles cloisonnées présentent des formes et des modifications qui leur sont individuelles et particulières. Les storilles 
sont assez rares, ils vivent arec une foule d'autres coquilles nicroscopiques, sur les bords du golfe Persique; et on en retrouve quelques espèces congénires prís du port de Livourne, dans la ci-devant Toscane: les cloisous du storille rayommant se dessinent encore sur une robeblanche et perlée, et c'est le diaphragme qui successivement forme ces cloisons, tandis que l'ouverture contre le retour de la spire paroit remplacer le trou du siphon; il est probable qu'ume partie du corps de l'animal y est engagíe de la même manière quel'est l'appendice filiforme des spirules: fait que nous arons de:ja observé dans quelques genres dont nous n'avons pu indiquer le siphon, et que nous retrouverons encore dans plusieurs des genres qui vont suivre. Quant au mamelon du sommet, on sait que beaucoup de coquilles univalves non-cloisonnées, en portent de semblables; ce qui indique que les formes changent, et que celles du premier âge ne sont plus les mêmes dans un âge plus avancé.

Le storille rayommant a quelquefois une ligne et demie de diamitre. 
Coquille à spire en disque, ombiliquée. 


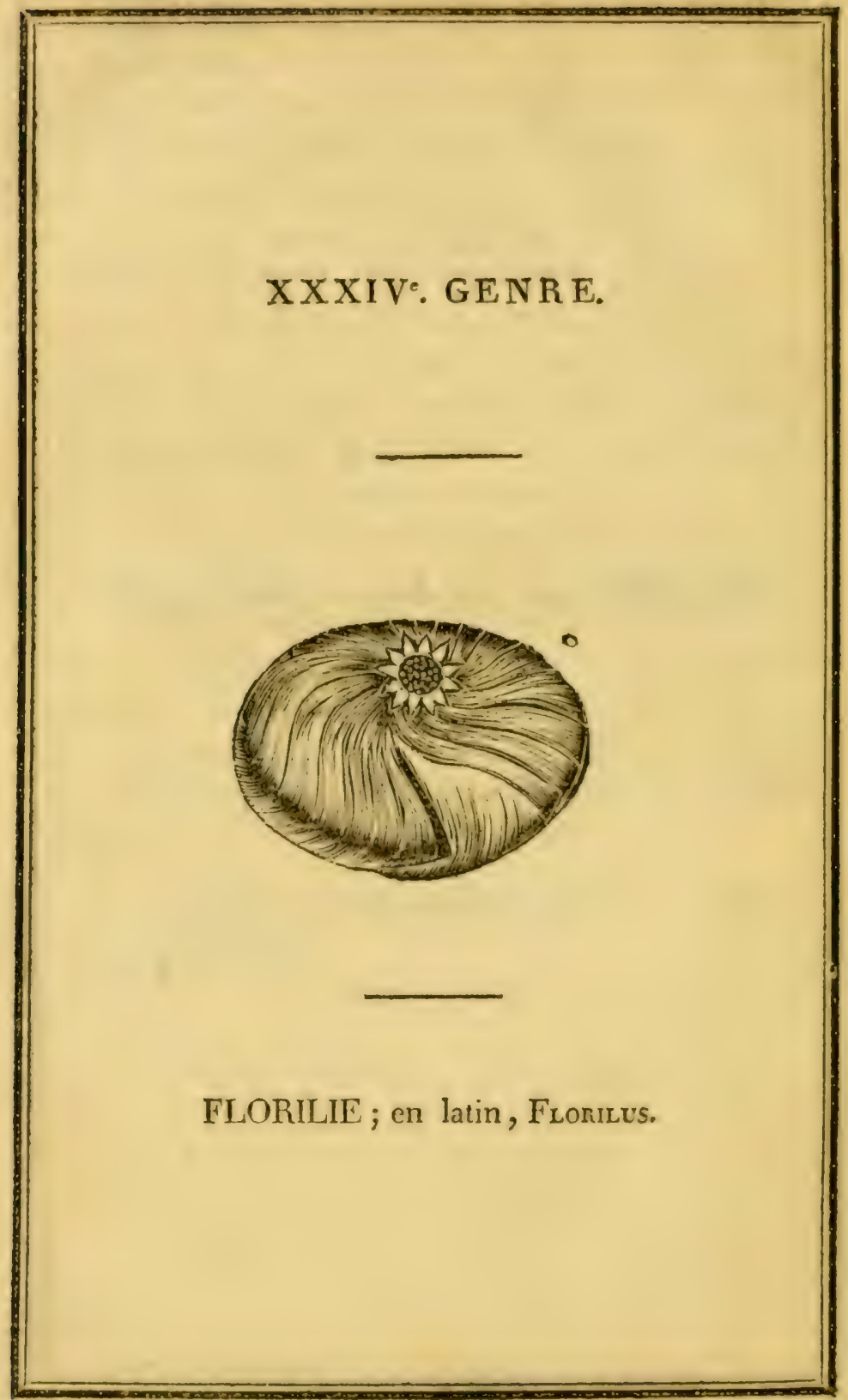




\section{LE FLORILIE.}

Caractères génériques. Coquille libre, univalve, cloisonnée, à sommet apparent et base ombiliquée; bouche triangulaire, recouverte par un diaphragme, et offrant une ouverture en ogive contre le retour de la spire, reçu obliquement dans cette ouverture; dos caréné, cloisons unies; siphon inconnu.

Espèce servant de type au genre.

Florilie étoilé. Florilus stellatus.

Nautilus asterizans, der gestirnte schiffer. Testac. microsc. a Leo. von Fichtel, et J. P.C. von Moll, tab. 3. fig. e-h.

Soldani testac. T. I. P. I. p. 66. tab. 6o. lit. B ?

En s'écartant des nautiles par son sommet et par son ombilic, ce genre rentre dans les coquilles turbinées cloisonnées; cet ombilic est assez profond, rond, et le dessous de la coquille est à-peu-près aplati ; le sommet est remarquable par un joli mamelon, étoilé dans cette espèce, et au centre duquel est un petit disque criblé de petits trous, ce qui donne à ce sommet un aspect 
1leuri, car il ressemble en petil au soleil du Pérou, ou tournesol.

La coquille est unie, finement striée, et les cloisons en sont très-apparentes; le dos est aigu ou caréné, mais non-armé, ct le retour de la spire n’a plus lieu médiatement dans le milieu de la bouche, attendu que la coquille est réellement turbinée. Sa couleur est jaune, striée de couleur de feu à l'ouverture des cloisons; elle est nacrée et diaphane.

On la trouve dans les algues, et dans les buissons des coralines de la Méditerranée, surtout dans la coraline de Corse.

Le florilie étoilé a une demi-ligne de diamètre. 
Coquille spirée, roulée sur elle-même. 


\section{$X X X V^{e}$. GENRE。}

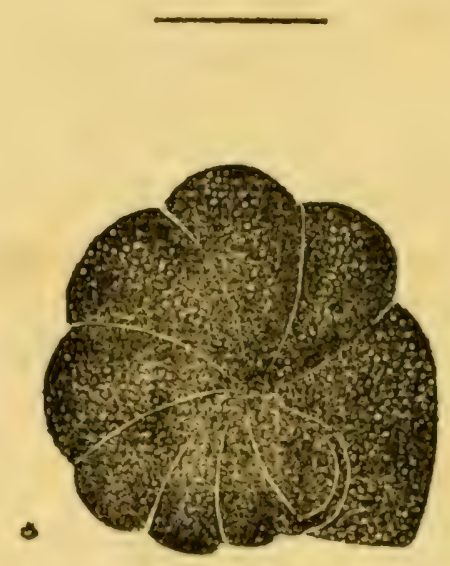

POLIXENE; en latin, Polyxenes. 


\section{LE POLIXENE.}

Caractères génériques. Coquille libre, univalve, cloisonnée, à sommet et à base ombiliquée, roulée sur elle-même; bouche linéale contre le retour de la spire; cloisons unies.

\section{Espèce servant de type au genre.}

\section{Le Polixène criblé. Polyxenes cribratus.}

Nautilus farctus. Der ausgestopfte schiffer, testac. microsc. a Leo. von Fichtel, et J. P. C. von Moll , pag. 64, tab. 9, fig. g, h, i.

Ce genre est encore un de ceux dont la base est aplatie, mais ici le sommet s'enfonce et n'est plus relevé ou saillant; dans cette coquille chaque concamération est arrondie en dòme; la bouche longue et linéaire est contre le retour de la spire, et individuellement elle est entièrement criblée de pores qui sont à jour ; organisation très-digne de remarque, et qui sembleroit permettre le passage à autant de bras qui, comme ceux des polypes, saisiroient leur proie à l'instant où elle s'en approcheroit : analogie qui nous conduiroit aux animaux 
constructeurs des coralines. L'étude que nous en avous faite nous a prouvé que ces mollusques étendent, développent plusieurs bras, et que ces bras, malgré leur ténuité, sont armés de cupules, tout comme ceux des séches, des poulpes et des calmars.

Les cloisons du polixène criblé sont trés-apparentes, d'autant plus quelles forment la côte de melon; cette coquille est rare.

Elle est fossile jusquà présent, et vient de la Coroncine, pres de Sicme, dans la ci-devant 'Toscane, anjourdhui Etrurie; sa couleur est jaune, mais ocracéc; elle a une demi-ligne de diamétre.

I A demière concamération est beaucoup plus renflée, et beaucoup plus grande que toutes les autres. 
Coquille spirée, contournée sous son sommet. 


\section{XXXVIe. GENRE.}
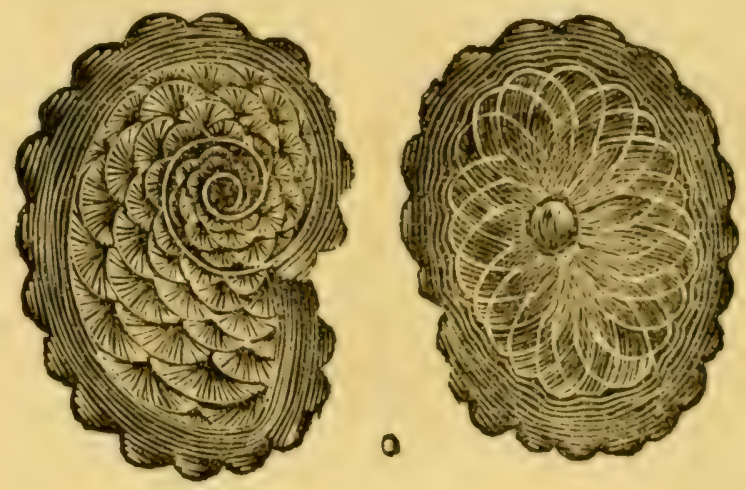

EOLIDE; en latin, Eolinr.s. 


\section{L'ÉOLIDE.}

Caractères génériques. Coquille libre, univalve, cloisonnée, à spire relevée et base aplatie; bouche ronde, placée au centre de la base; dos ou marge caréné et armé ; cloisons unies.

Espèce servant de type au genre.

\section{L'Éolideécaillé. AEolides squammatus.}

Polythalames de Soldani, Testac. tał. 167 . vv.

Pour faire connoitre parfaitement les formes de notre type, nous avons été forcé de doziner deux figures; l'une représente la coquille vue par-dessus, et l'autre par-dessous ; du côté du sommet on voit le têt se dessiner en écailles de poisson: il est bordé de sa carène ondulée en armature, et sa spire saillante, quoiqu'arrondie, est parfaitement régulière: la base est un peu renflée et vésiculaire, elle est marquée d'un réseau circulaire, se terminant en ogive contre la carène, et les striess'en dessinent finement en cerceaux.

Au centre de la base est l'ouverture ou bouche; elle est ronde, assez petite, et elle indique que l'animal qui occupe cette coquille a une grande portion de son corps à l'extérieur, qui, comme dans les porcelaines et les ovules, 
recouvrantentièrement le dos de la coquille, ajoute continuellement à son épaisseur, et forme successivement denouvelles couchesà l'extéricur, et peut-être même les raies divergentes qui représentent sur ce têt les écailles de poissons.

Cette coquille cloisonnée est diaphane et transparente; sa couleur est jaune, ardente et irisée.

On la trouve surles rivages de la Méditerranée; elle a deux tiers de ligne de diamètre. 
Coquille en disque, et lenticulaire, à spire intérieure. 


\section{XXXVII'. GENRE。}

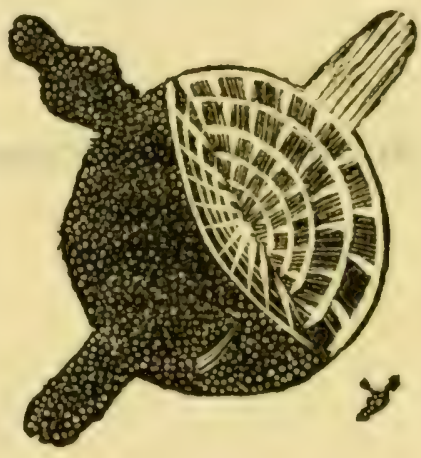

TINOPORE; en latin, Tixoronus: 


\section{LE TINOPORE.}

Caractères génériques. Coquille libre, univalve, cloisonnée et cellulée, spirée et lenticulaire ; têt granulé extérieurement ; bouche sémi-lunaire, placée vers la circonférence et sur un des côtés ; dos caréné, armé de quatre pointes au plus; les deux centres bombés et relevés.

Espèce servant de type au genre.

Le Tinopore bâtonné. Tinoporus baculatus.

Nautilus Spengleri, der spenglerische schiffer. Testac. microsc. a Leo. von Fichtel et J. P. C. von Moll, pag. 89. tab. I5. fig. i. k. Quatrième variété.

Cette coquille, qui pour nous est la tête d'un genre nouveau etassez nombreux, ressembleroit à la numulie ou camérine tuberculée et criblée, si elle n'étoit armée de trois pointes obtuses. Ces pointes sont intérieurement sillonnées et tuberculées à la manière de quelques tubipores : la bouche de la coquille, placée sur un des côtés, est très-remarquable, en ce qu'elle est petite et formée en demi-lune : la spire est cachée et intérieure. Les auteurs allemands que nous avons cités dans notre synonymie, y comptèrent au moins quatre-vingts cellules. Nous avons fendu cette coquille à demi par le milieu, afin de faire apercevoir la construction de l'in- 
L'́ricur, qui, cellulé sur divers plans, nous conduit naturellement aux numulies, mais elle en differe par ses bras ou pointes, qui sont constans, quoique leur intérieur présente une organisation qui n'est point celluléc, mais tubulée.

La couleur du tinopore baculé est blanclie, flambée et teintée de jaune; la coquille est entièrement opaque.

L'individu qui a servi de sujet à notre description venoit de la mer des Indes orientales; on le trouva dans le sable dont étoit remplie une coquille du genre casque: on rencontre encore les tinopores parmi d'autres coquilles microscopiques, sur les plages du golfe arabique, ainsi que dans quelques éponges de la mer Adriatique.

D'une pointeà l'autre le tinopore que nous venons de décrire, a deux lignes de diamètre. 
Coquille en disque, et lenticulaire, à spire intérieure. 
XXXVIII* GENRE.

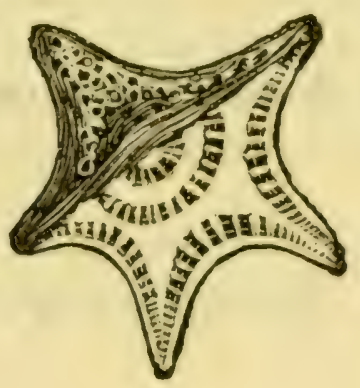

SIDÉROLITE; en latin, Sinerourtes. 


\section{LE SIDERROLITE.}

Caractères génériques. Coquille libre, univalve, cloisonnée et cellulée, lenticulaire, tuberculeuse sur les deux șommets; marge carénée, éperonnée ou armée; bouche inconnue.

\section{Espèce servant de type au genre.}

Sidérolite chaussetrappe. Siderolites calcitrapes.

Siderolithes calcitrapoüdes. De la Marck, Syst. des anim. sans verteb., pag. 376 . Knorr. Pétrif. $3^{\mathrm{e}}$. vol. suppl. pag. I8 г. fig. 9-16. Faujas-de-Saint-Fond, Hist. nat. de la mont. de St.-Pierre, à Maëstricht.

Faujas-de-Saint-Fond, est le premier auteur qui, en faisant voir l'intérieur de cette coquille, a prouvé que ce n'étoit point un polypier pierreux à rayons, ou madrépore des zoologues; de la Marck, d'ailleurs si exact et si didactique, paroît en conséquence être en erreur lorsqu'il en fait son $\mathrm{XX}^{\mathrm{e}}$. genre des polypes à rayons. Dans les sidérolites, indépendamment du sys- 
téme des cellules centrales et spirales, il semble qu'il en existe un second qui suit la direction des pointes disposées en molette d'éperon; ce qui les distingue des tinopores dont nous avons formé le genre précédent : nous avons de mème dessiné ici le sidérolite avec un quart de son têt enlevé, afin de laisser voir la disposition des cloisons et des cellules. Il y a des sidérolites qui ont de trois à quatre lignes, et mème plus, de diamètre, et quelques-uns ont jusqu’à huit et dix pointes.

On ne les connoît encore qu'à l'état fossile, et on en a rencontré une grande quantité parmi les pétrifications si nombreuses de la montagne de St.-Pierre, à Maëstricht.

Leur couleur est jaune, ocracée, ferrugineuse ; leur substance calcaire et crayeuse; ils sont opaques et solides. 
Coquille en disque, et lenticulaire, à spire intérieure. 


\section{XXXIX}

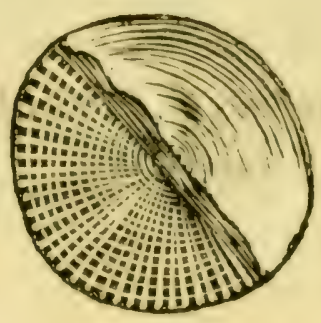

NUMULIE; cn latin, Numulites. 


\section{LE NUIULIE.}

Caractères génériques. Coquille libre; univalve, cloisonnée, cellulée et lenticulaire; têt extérieur uni; spire intérieure ; bouche inconnue ; dos ou marge caréné ; les deux centres bombés et relevés.

\section{Espèce servant de type au genre.}

\section{Le Numulie monnoyé. Numulites denarius.}

Nummus diabolicus, nummus sancti Petri, sancti Bonifacii, lapides numismales; en allemand, Teufelspfennige, schwefelkiese; en belge, Sint Pieter's munt, duivels munt. D'Argenville, oryct. pl. 8. fig. 10. Bourguet, petrif. tab. 5o. fig, 32 I , 32 . Guettard, mem. 3. pag. 33 I. tab. I3. fig. x-1 0. Knorr, foss. I I. tab. A. VII. no. I-I 2. Brugniere, encyclop. nummulites lævigata. De la Marck, hist. des anim. sans vert. pag. 181. Genre LXXXIX. Felix de Roissy, hist. des moll. Buffon, edit. de Sonnini, tom. 5, pag. 49 et suivantes.

Comme pétrification, cette coquille a été très-fréquemment citée par tous les oryctologues, tantôt sous les dénominations que nous venons de rapporter, et tantôt sous les noms de camérine, d'hélicite, de pierres lenticulaires, de numismales, de monnoie de St.-Pierre, de monnoie du diable, de placites, de porphites, de discolithes. Agglomérée, c'est le lapis frumentarius de Langius, ou pierre de froment, parceque tranchées dans leur plus petit diamètre, leur coupe ressemble un peu par la forme à un grain de blé. 
Les numulies sont disséminés à l'état de pétrification dans les bancs calcaires du globe entier, quelquefois ils forment a eux seuls de grands pays; base du rocher lybique, c'est sur eux que sont fondées les pyramides d'Egypte.

Nous avons encore enlevé une partic du têt de celui ' fue nous présentons, afin de faire entrevoir la disposition des cellules intéricures. Il est probable que Stavorinus a décrit dans son Voyage aux Indes, l'animal des numulies; mais comme ce navigateur hollandois n'en a point donné de figure, il existe encore du doute a cet égard. Quant à l'animal que M. Péron, naturaliste de la seconde expédition autom du monde, commandée par le capitaine Baudin, a donné pour l'analogue du numulie que nous décrivons, nous avouons de bonne foi que nous n'avons pu l'y recomnoître. On trouve des numulies fossiles de diverses grandeurs, depuis le diamètre d'un écu jusquà celui d'un point mathématique. Von Fichtel et von Moll en ont décrit plusicurs espèces sous le nom de Nautilus mamilla, et comme ils ne traiterent que des coquilles microscopiques, il paroit que c'est en raison de leur grandeur que ces auteurs ont voulu les ranger parmi les nautiles; car on ne peut soupçonner que les nummulites ou camérines leur fussent inconmues; on peut consulter à cet égard leur ouvrage, pag. 55. tab. 6. fig. $a, b, c, d$. 
Coquille en disque, et lenticulaire; à spire intérieure. 
XLe. GENRE.

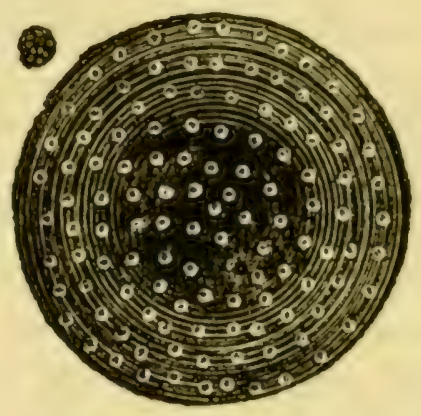

LICOPHRE; cn latin, Lycopniss. 


\section{LE LICOPHRE.}

Caractères génériques. Coquille libre, univalve, cloisonnée et cellulée, lenticulaire ; têt extérieurement tuberculé ou criblé, sans rides ou rayons; recouvrant la spire intérieure; bouche inconnue, dos ou marge caréné; centres bombés et relevés.

\section{Espèce servant de type au genre.}

\section{Licophre lentillé. Lycophris lenticularis.}

Nautilus lenticularis, der linsenformige schiffer, testac. microsc. a Leo. von Fichtel, et J. P. C. von Moll, pag. 56. tab. I 7 . fig. $a-b$, deuxième variété.

Nous ne connoissons encore cette coquille qu'à l'état fossile, elle est une de celles qu'on trouve dans les bancs calcaires de la Transylvanie en très-grande quantité, et son entassement fait présumer que les licophres sont, comme les numulies, des mollusques qui vivent en grandes familles. Jusqu'aux auteurs allemands que nous venons de citer, on les avoit regardés comme une espèce de numulie, mais MM. von Fichtel et von Moll les ayant retirés de ce genre pour les donner aux nautiles, nous croyons, avec encore plus de raison, pouvoir en faire un genre particulier, d'autant plus qu'ils sont diaphanes et criblés, pour ainsi dire, à jour, ce qui rend leurs cellules rondes, et il seroit même possible de regarder chaque trou comme une bouche, d'autant plus qu'elles paroissent s'être fermées successivement. 
Nous ignorerons encore long - temps le mode d'être d'une foule de coquilles microscopiques, parce qu'en général elles échappent à nos regards, que leur étude ne fait, pour ainsi dire, que de naitre, et que leur observation est bien plus difficile que celle des mollusques testacés plus grands, qui cependant sont loin encore d'être bien connus; mais les formes des coquilles microscopiques et leurs aspects nous conduisent à reconnoitre ou à soupçonner une longue série de mollusques testacés, soit rampans, soit flottans, et intermédiaires entre ces mollusques, les polypes proprement dits, et les animaux des madrépores ; c'est pourquoi nous avons placé dans cet ourrage un assez grand nombre de ces coquilles microscopiques si négligées, comme formant des pierres d'attente sur lesquelles on pourra båtir un jour.

Le licophre lenticulaire a près de trois lignes de diamètre ; on le trouveà Claudiopolis, dans lit Transylvanie; sa couleur est ferrugineuse ou variée d'après les bancs quile renferment; et quelquefois mème la coquille diaphane a encore conservé sa teinte nacrée. 
Coquille en disque, et lenticulaire; à spire intérieure. 

I6.
COQUTLLS

XLI'. GENRE.

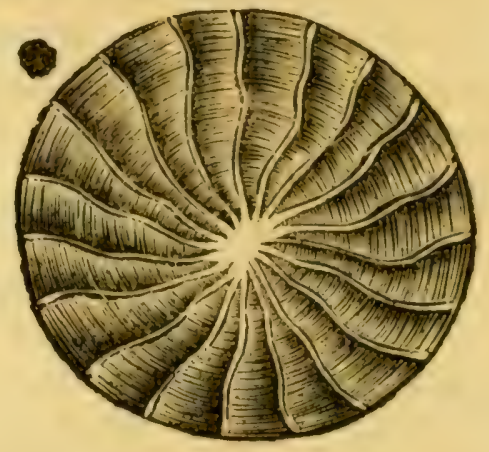

ROTALITE; en latin Rotalites. 


\section{LE ROTALITE.}

Caractères génériques. Coquille libre, univalve, cloisonnée et cellulée, lenticulaire; têt extérieurement striée et ridé en rayons, recouvrant la spire intérieure; bouche inconnue; dos ou marge caréné; centres bombés et relevés.

Espèce servant de type au genre.

Rotalite rayonnant. Rotalites radiatus.

Hélicite rayonnée. Guettard. mem. vol. 3. pag. 432. pl. XIII. fig, r I-22.

Testac. microsc. a Leo. von Fichtel, etc., pag. 57 , tab. 7, fig. 9. De la Marck, Syst. des anim. sans vert. addit. pag. 40r. Felix de Roissy. Moll, edit. du Buffon de Sonnini, vol. 5 , pag. 59 .

Ce genre rotalite est déjà formé par d'autres conchyliologues que nous, et quoique nous ayons conservé cette dénomination, notre phrase caractéristique n'est point la même, parce qu'ils ont donné à leur rotalité une petite bouche marginale et triangulaire, en citant pour type une espèce presque microscopique qui se trouve fossile à Grignon, près Versailles. Nous avouons que nous n'avons point pu reconnoître cette bouche, et nous soupçonnons qu'on aura regardé comme telle une fracture que la coquille présentoit sur son dos ou marge: et quoique ces auteurs aient reconnu pour caractère tranchant un seul côté à sommet tuberculé, tandis que l'autre seroit lisse, nous ne doutons pas cependant que 
cette espéce de Grignon, (qu'ils ont décrite, n’appartienne à notre genre, ou peut-chte à celui que nous metrons immédiatement à sa suite; car nous avons cru entrevoir que les figures de Guctard, que De la Marck cite pour synonymie, ont été regardées par d'autres auteurs, et notamment par Fortis, dans ses mémoires sur l'Ttalic, et par Felix de Roissy, comme simples variétés, mais strices, de la camérine on numulic : et pour ne point multiplier les objets et les dénominations sans n'ecessité, nous avons'conservé le nom imposé au genre par De la Marck, don nous nous honorons d'aroir suivi les leçons au Muséum d'histoire naturelle.

Le rotalite rayonnant se trouve fossile en Suisse, en Allemagne, en Hongrie, et surtout en Transylvanie; il a deux lignes de diamétre, et partage la couleur des couches dans lequel il gît à bancs pressés. 
Coquille en disque, et lenticulaire ; à spire intérieure. 


\section{GENRE。}

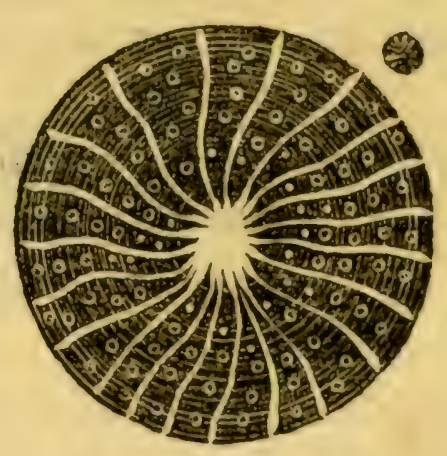

L'GÉONE; en latin, EGEON. 


\section{L'ÉGÊONE.}

Caractères génériques. Coquille libre, univalve, cloisonnée et cellulée, lenticulaire ; têt extérieurement strié et tuberculé ou criblé en rayons, recouvrant la spire intérieure; bouche inconnue; dos ou marge caréné; centres bombés et relevés.

\section{Espèce servant de type au genre.}

Égéone perforé. Egeon perforatus.

Nautilus lenticularis, der linsenformige schiffer. Testac. microsc. a Leo. von Fichtel, etc., pag, 57 , tab. 7 , fig: $h$, cinquième variété.

Il n'a encore été rencontré qu'à l'état fossile; les stries ou rides qui garnissent les deux côtés de cette coquille partent du centre et vont se rendre en divergeant vers la circonférence; elles alternent avec des rangées intermédiaires de tubercules, ou d'espèces de trous disposés en séries. L'égéone perforé vient de Claudiopolis, en Transylvanie, ou , entremêlées en grande quantité avec des numulites, ces coquilles pressées et entassées forment presque le sol entier, au point qu'elles le rendent, partout où elles sont, entièrement stérile.

L'égéone est diaphane, ses côtes sont plus opaques que le têt, et les trous dont il est criblésont plus clairs. Sa cou 
leur est ordinairement blanche, encore un peu perlée; d'autres fois ocracéc et ferrugineuse, en raison des bancs où il se rencontre; il y ena méme qui forment des mines de fer en pleine exploitation. L'égéone perforé a deux lignes de diamètre.

Malgré l'usage, nous avons donné à des coquilles dont les noms ont une terminaison féminine, une acception masculine; et nous nous y sommes décidés d'autant plus volontiers que cette seule acception dílivre de l'embarrás d'avoir à chercher de nouvelles dénominations pour désigner les animaux de ces corfuilles lorsqu'ils nous sont connus; c'est ainsi qu'au lieu de dire un nautilier, un océanier, un mólonier, un ammonier, nous dirons un nautile, un océanie, un mélo nie, un ammonie, etc., marche qui nous a paru plus simple, et propre d'ailleurs à lever beaucoup de diffi. cultés. 
Coquille en disque, et globuleuse; à spire intérieure. 
COQUIIL ES

\section{GENRE.}

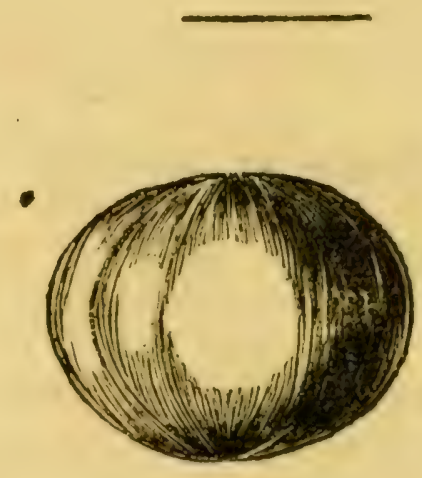

BORÉLIE; en latin, Borelis. 


\section{LE BORÉLIE.}

Caractères génériques. Coquille libre, univalve, cloisonnée et cellulée, globuleuse ; le têt extérieur formé en côtes de melon, et recouvrant la spire intérieure; bouche inconnue; sommets déprimés.

\section{Espèce servanit de type au genre.}

Borélie melonné. Borelis melonoïdes.

Nautilus melo, der melonenschiffer, testac. microsc. a Leo. von Fichtel, etc., pag. 123, tab. 34 , fig. 9 , variété 2 .

Ce sont ces coquilles, d'autres qui leur sont congénères, et d'autres encore de genres différens quil ne faut cependant point confondre avec les meconnites ou mines de fer en graines de parots, qui forment quelquefois à elles-seules des bancs entiers de pierre calcaire. D'autres fois elles constituent par leur grand nombre l'empâtement ou gangue de masses de pétrifications plus grandes. Les anciens oryctologues les ont nommées oolithes, cenchrites; en allemand, roggensteine;pierres de frais, œufs de poissons. On trouve beaucoup de ces pierres formant des bancs, en Suisse, dans le territoire d'Halberstadt et près de Querfurth en Allemagne. Le borélie melonnése trouve à Grignon près de Versailles, à Courtagnon près de Rheims, et à Chaumont dans le Vexin françois, principalement au mont Thouin; sa 
couleur est blanche, quelquefois un peu rosće, souvent ocracée; il a une demi-ligne de diamètre.

On le trouve encore dans les sables des environs du villée d'Alpestis, dans le comté d'Hunniade, en Transylvanie; dans cet endroit ces coquilles sont jaunes et ocracées.

On peut consulter, à l'égard de ces fossiles, Bertrand ( I), Heuckel (2), Kundmann (5), Bruckmann (4), Buttuer (5), Volckmanu (6), Scheuchzer (7), Licbkuecht (8), ainsi que beaucoup d'autres auteurs.

(r). Dietionuaire des fossiles.

(2) Flora saturnizans, pag. 538.

(3) In rar. nat. et art.pag. 147 ; et onomnt. hist. nat. tom. I. pag. 349.

(4) Ilist. nat. oolithi, seu ovariorum piscium et concharum in saxa mutatorum. Helmstadt, I6 6 I.

(5) De ruderibus dilusii testibus, pag. $2 / 55$.

(6) Silesia subterranea, tab. $26, n^{\circ}, 19$.

(7) Naturgeschichte der Schweitz. pr. I. pag. 106.

(8) Specimen Hassix subter.'pag. 97. 
Coquille en disque, ovulaire; à spire intérieure. 


\section{XLIVe. GENRE.}

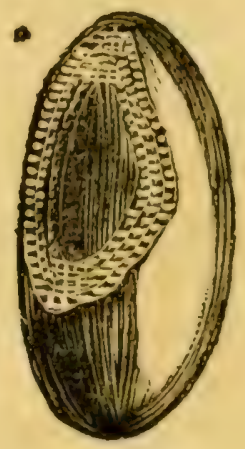

MILIOLITE; en latin, Minor.rtes. 


\section{LE MILIOLITE.}

Caractères génériques. Coquille libre, univalve, cloisonnée et cellulée, ovulaire ; lisse extérieurement; spire cachée et intérieure ; bouche inconnue; sommets élevés.

\section{Espèce servant de type au genre.}

Miliolite sablonneux. Miliolites sabulosus.

Comme la coquille précédente, on trouve le miliolite, mais en moindre quantité, dans les environs de Versailles, en Champagne, dans le Vexin françois, et en quelques autres endroits encore, romme dans le Falun de Touraine, où, confondu avec une foule d'autres corps microscopiques, il remplit les interstices et les cavités de coquilles plus grandes: le nombre de ces petites coquilles y surpasse limagination.

On rencontre encore le miliolite dans les environs de Paris, au milieu d'une pierre calcaire d'un ton gris, dans la pâte delaquelle il est renfermé, et on ne doit pas confondre cette coquille cellulée avec l'ovéolite de Lamarck (Syst. des anim. sans vert. add. pag. 402.), car elles n'ont entr'elles aucune analogie, l'ovéolite étant uniloculaire, c'est-à-dire sans aucune cloison, ni cellule, et d'ailleurs percéà son sommet. 
Le miliolite sablonneux est blanc, oparyue, quelquefois jaunissant ou ocracé; il a une ligne dans son plus grand diamètre.

Nous avons brisé trois de ses circonvolutions, afin de faire voir la disposition de ses cloisons et de ses cellules intéricures; cette organisation est la même que celle des tinopores et des numulies, à la suite desquels ce genre est venu se placer, après avoir cependant été précédé par quelques intermédiaires. 
Coquille en disque, globulaire; à spire intérieure. 


\section{XLV*, GENRE.}

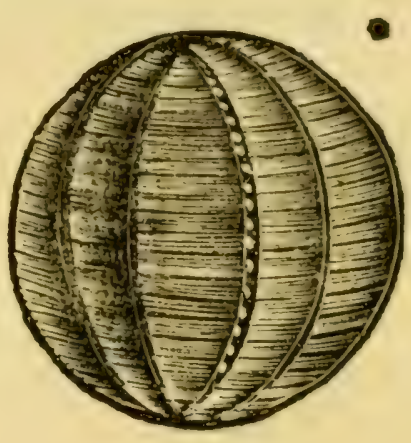

Clausulie; en latin, Clausurus. 


\section{LE CLAUSULIE.}

Caractères génériques. Coquille libre, univalve, cloisonnée et cellulée; globulaire et contournée en spirale; le dernier tour de spire renfermant tous les autres; bouche sériale, cellulée, étroite, de toute la longueur de la coquille, et recevant en plein le retour de la spire; cloisons unies et sériales.

\section{Espèce servant de type au genre.}

Le Clausulie indicateur. Clausulus indicator.

Nautilus melo, der melonen-schiffer, testac. microsc. a Leo. von Fichtel, erc. , pag. i 8 , tab. 24 , fig. a-f.

Joh. Ehr. von Fichtel Nachricht. von den Versteinerungen Siebenbürgens, etc., Nurnberg. i 780 . P. I. pag. 78.XXIII. Il le donna pour une espèce d'échinite.

Le clausulie est fait en globe parfait; il a un axe comme les autres coquilles que nous venons de décrire; mais ni l'une ni l'autre de ses extrémités ne peuvent être regardées comme sommet: le têt offre des côtes en longueur, et des stries en travers, et la coquille sembleroit être formée par autant de tuyaux roulés en spirale, et latéraux les uns aux autres. On peut les suivre isolément depuis l'ouverture particulière qu'ils offrent à la bouche commune jusqu'à la naissance de la spire au centre de la coquille. Chacune de ces ouvertures de la bouche est faite en gueule de four, et ces ouvertures sont constamment en nombre pair ; les côtes indiquent les cloisons qui font de ces tuyaux autant de coquilles cloisonnées; et par leur réunion, une coquille cellulée. 
Malgre cette structure singuliere, qui semble appartenir at plusieurs genres, mais que nous avons remis à faire comnoitre lorsque nous parlerions des clausulies, ce genre actuel n'appartient pas à coup sûr à des polypes, maisbien à des mollusques conchiliferes. Il tranche donc la question, et nous avons cru pouvoir domer au clausulie qui nous sert de type, l'épithète d'indicateur. Dëjà admis parmi les coquilles par les auteurs allemands si exacts que nous nous plaisons à citer, ce genre jette un grand jour sur toutes les coquilles cellulées, mais dont la bouclie est inconnue.

La couleur du clausulic indicateur est tantót blanche et tantòt ferrugineuse et ocracée ; jusqu'à présent on ne les a rencontrés qu'à l'état fossile.

Blancs, à Brumn, et à Stcinfeld, sur la rivière de la Hongrie et de l'Autriche inférieure, oi ils sont rejetés avec d'autres pétrifications par une fontaine qui source du scin d'un lac. - De la même couleur dans les pierres calcaires de Kroisbach, près du lac de Heuscieder en Hongrie; mêlés arec une foule de nautilites et de pectinites. - De même dans les pierres dont a été construite au commencement du dernier siècle la chapelle de Ste.Madeleine à Vienne en Autriche; pierres probablement tirées de quelque carriére voisine, maintenant incomme.

Bruns et ocracés, dans le sable quelquefois cohérent du village d'Alpestis, dans le comté d'Humiade en Transylvanie.

Blancs et rosés, dans la pierre puante noire de Duind sur le bord de la mer Adriaticque.

Leur axe a quelquefois une ligne de diametre. Il y a une espéce dont le têt est uni. 
Coquille en disque, globuleuse; à spire intérieure. 


\section{XLVI: GENRE.}

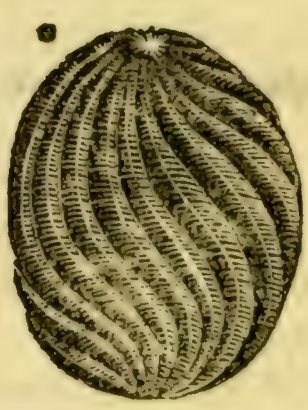

GIROGONITE; en latin, Grnogovires. 


\section{LE GIROGONITE.}

Caractères grénériques. Coquille libre, univalve, cloisonnée, globuleuse et ovoïde ; têt sillonné à l'cxtérieur; spire cachée ou intérieure; bouche inconnue; cloisons unies, contournées en double crochet ou $S$; sommets arrondis.

\section{Espèce servant de type au genre.}

Girogonite luzerné. Girogonites medicaginula.

De la Marck, Syst. des anim. sans vert. add.pag. $40 \mathrm{r}$.

Cette petite coquille est répandue avec la plus grande profusion dans tous le bassin de la Seine, autour de Paris, où on la trouve dans la masse d'une pierre dure, faisant feu avec le briquet et siliceuse, quoique la coquille ait conservé son têt à l'état calcaire. Ordinairement ce silex se trouve par fragmens, ou rognons, et pierres roulées, et sa couleur est d'un gris tirant sur le blanc; il est très-dur.

Le girogonite luzerné a une configuration qui lu: est particulière, en ce que chacune de ses concamérations peut se détacher : en cet état elles ressemblent à des graines de luzerne, et paroissent fermées de tous 
culés, comme le seroit une gousse de pois. Ces concamérations font le double crochet, elles sout renflées dans le milieu, minces sur le côté qui tient au centre de la coquille, pointues aux deux extrémités, et carénées en crête assez aigue au côté formant le têt extéricur.

La couleur de l'espèce que nous décrivons estblanche, quelquefois jaunatre; elle a une demi-ligne de diamètre.

La configuration des concamérations donne à l'extérieur de crue jolie coquille un aspect tourmenté et guilloché. Chacune de ses côtes est très-saillante. 
Coquille en disque aplati, à spire intérieure. 


\section{XLVIIe, GENRE。}

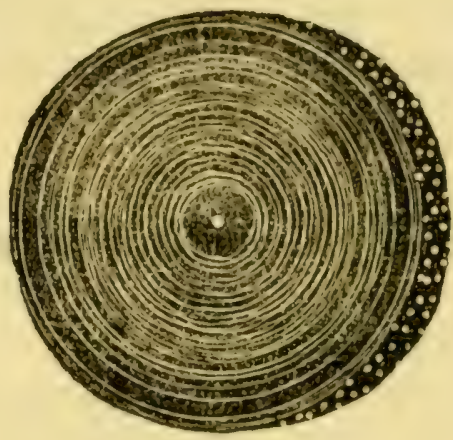

DISCOLITE; en latin, Discolites. 


\section{LE DISCOLITE.}

Caractères génériques. Coquille libre, univalve, cloisonnée et cellulée; en disque et aplatie; très-mince au centre, plus épaisse sur les bords; le dos ou marge entièrement recouvert d'un diaphragme criblé de pores; bouche inconnue.

Espèce servant de type au genre.

Discolite concentrique. Discolites concentricus.

Il est assez étonnant que les conchyliologues modernes n'aient point parlé de cette coquille fossile, qu'on trouve cependant si fréquemment parmi celles de Grignon et de Courtagnon; il est vrai qu'elle est extrêmement fragile, et que pour peu qu'on la touche, elle se sépare en cercles que l'on croiroit concentriques, mais qui sont des portions de spirale; et comme en général il en est d'assez grands, on auroit pu croire qu'elles auroient attirés davantage l'attention. Le discolite est d'autant plus singulier, qu'on ne peut lui donner d'autre bouche que son tour tout entier, qui est criblé de pores ou petites ouvertures; la spire devient plus grande et plus épaisse à mesure que la coquille prend plus d'accroissement. Nous en avons vu d'un pouce de diamètre, mais le milieu reste constamment plus mince qu'une feuille de papier. 
Le célébre abbé Fortis, qui nous honora de son amitié ct que la mort est venu enlever aux sciences, dans un âge à la vérité assez avancé, avoit donné le nom de Discolithes (I) à tous les corps marins quil rangeoit parmi les numismales ou numulies; mais comme nous avons conservé l'ancienne dénomination, nous avons cru pouvoir faire revive celle de discolite en faveur du nonveau genre, dont les coquilles sont constamment en disque aplati.

La couleur du discolite concentré est blanche, quelquefois ocracée : nous en connoissons une autre espèce, mais dont la superficie et guillochéc.

(1) Fortis, Mémoires sur l'Italic.: 
Coquille en disque aplati, contournée en spirale. 


\section{GENRE。}

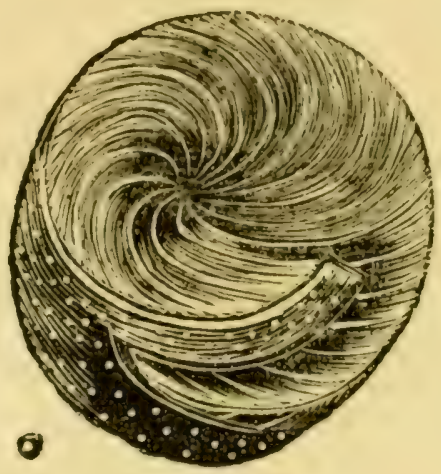

ARCHIDIE; en latin, Ancmaras. 


\section{L'A R CHIDIE.}

Caractères génériques. Coquille libre, univalve, cloisonnée et cellulée; en disque et aplatie; spire excentrique, ombiliquée; dos caréné ; bouche aplatie, triangulaire, très-allongée, au moins d'un demi-contour de la coquille, recevant dans son milieu le retour de la spire, et recouverte par un diaphragme criblé de pores; cloisons unies et criblées; le dernier tour de spire recouvrant tous les autres.

\section{Espèce servant de type au genre.}

Archidie spirant. Archaias spirans.

Nautilus angulatus, der winkelige schiffer, testac. microsc. a Leo. von Fichtel, etc., pag. I13, tab. 22, fig. a-e.

La bouche si particulière de cette coquille, et qu'on pourroit appeler dorsale, sert à nous donner une idée de la grande variété de formes que l'on rencontre chez les mollusques testacés ou conchilifêres. De concert avec le genre précédent, celui-ci nous mène de même aux polypiers : tous deux font la nuance intermédiaire. Cette bouche s'élargit avec l'àge, elle fait la fourche sur le retour de la spire, son diaphragme ressemble à une aire 
criblée, mais comme elle finit en pointe, le dos de la coquille est constanment caréné : l'archidie spirant gauchit un peu, l'origine de la spire n'ítant pas au milieu, et on pourroit y soupconner un sommet, l'ombilic étant mieux marqué sur une des surfaces: l'intérieur est divisé en un grand nombre de cellules qui augmentent en nombre avec les tours de spire, comme nous le prouvcrons par une section lorsque nous parlerons de l'hélénide.

La couleur en est blanche.

On trouve l'archidie spirant dans le golfe arabique, et Spengler est le premier qui l'y a découvert, c'est lui qui en fit le don à d'autres conchyliologues.

Cette coquille a presque une ligne de diametre. 
Coquille en disque aplati, contournée en spirale. 
XLIX: GENRE.
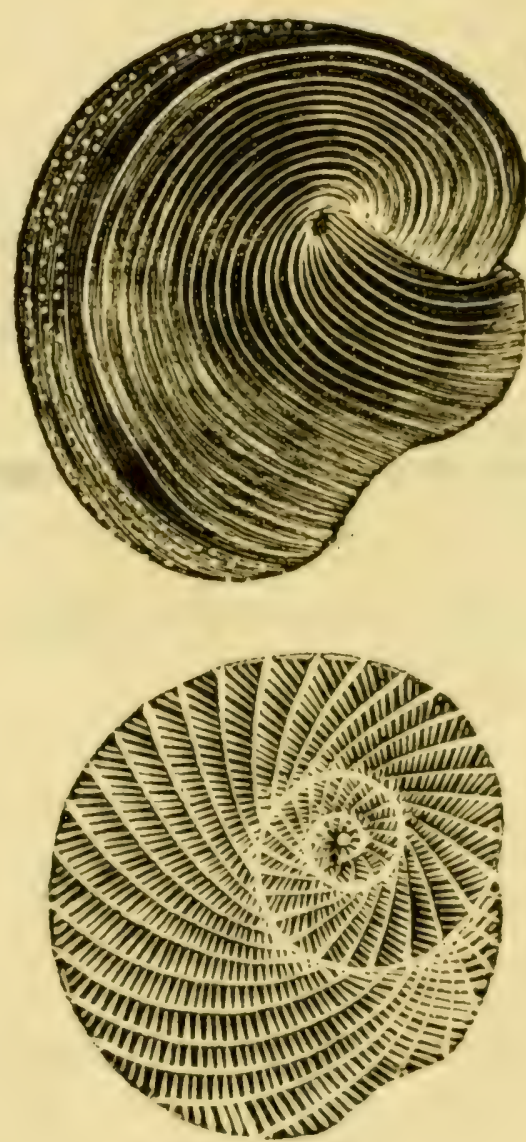

HÉLÊNIDE; en latin, Hrc.xis. 


\section{L'HÉ LÉ NIDE:}

Caractères génériques. Coquille libre, univalve, cloisonnée et cellulée, contournée en disque aplati; spire apparente, excentrique sur les deux flancs ; dos caréné ; bouche très-alongée, recouverte par un diaphragme criblé de pores; cloisons criblées et unies.

Espèce servant de type au genre.

L'Hélénide épanoui. Helenis spatosus.

Nautilus aduncus. Der eingekriinmte schiffer. Testac. microsc. a Leo. von Fichtel, etc., pag. I 15 , tab. 23 , fig. a.

Le genre que nous venons de caractériser a beaucoup d'analogie avec le précédent; mais la coquille qui nous sert de type n'est point ombiliquée, et sa bouche ne reçoit pas le retour de la spire, qui vient au contraire s'appuyer contre elle. Les deux flancs relevés de l'hélénide vont nous conduire à plusieurs genres de coquilles qui , mamelonnées sur les flancs, rentreroient cependant pour leurs formes dans les nautiles, si d'un autre côté leurs bouches ne venoient pas essentiellement les en séparer.

Les stries de l'hélénide épanoui vont dans le sens des côtes ou cloisons, et elles sont finement recroisées par d'autres qui marquent la cellulation; à mesure que la coquille croîtet augmente, elle forme l'aile ou la trompe en s'écartant du retour de la spire, avec lequel néanmoins elle fait corps, et c'est cet écartement qui nous a décidé à lui donner l'épithète caractéristique d'épanouie 
ou d'épatée. Les cellules de cet hélénide sont extrèmement nombreuses, on peut en compter plus de six mille. La planche qui fait partie de cet article présente deux figures, la premiére ou supérieure est celle de la coquille entiere, et la seconde offre sa coupe sur le plan de son plus grand diamètre : par cette section nous avons roulu domner une idée de la disposition intéricure de la spire et des cellules, disposition qui est commme à plusicurs autres genres, et notamment aux archidies. Les rangs de ces cellules en offrent davantage a mesure qu'ils devicnment plus longs, par l'agrandissement de ces coquilles. C'est en examinant de prés cette organisalion, et d'apres des observations qui nous sont particulieres, mais qui n'ont point encore acquis toute 'la maturité nécessaire, que nous croyons pouvoir regarder les hélénides, les archidies, les discolites comme servant de têt et de demcure à autant de familles de mollusques, vivans en société, mais différens des animaux des polypiers; plus rapprochés des sèches, des poulpes et des calmars que des polypes, et donnant toutes les nuances internćdiaires; nouvel anneau de cette concaténation générale qui lie insensiblement tous les êtres les uns aux autres.

La couleur de l'hélénide épanoui est blanche; il se trouve au golfe d'Arabie; Spengler le découvrit dans le sable qui remplissoit de plus grosses coquilles.

Il a deux lignes dans son plus grand diametre. 
Coquille en disque aplati, mamelonnée, contournée en spirale. 

198
COQU I Y E 5

\section{I: GENRE。}

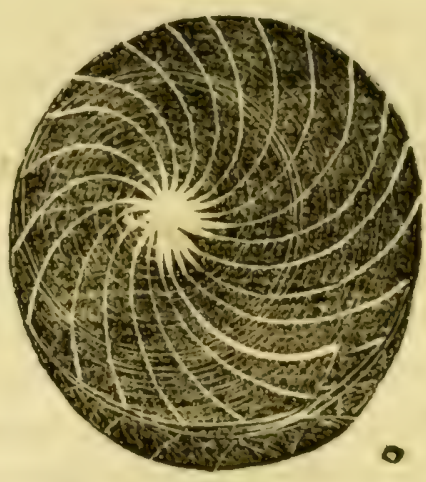

ILOTE; en latin, ILOTES. 


\section{L'ILOTE.}

Caractères génériques. Coquille libre, univalve, cloisonnée et cellulée, contournée en disque, et presque lenticulaire; spire excentrique, apparente, mamelonnée sur les deux flancs ; bouche linéale, triangulaire, échancrée sur le dos et cellulée, recevant dans son milieu le retour de la spire; le dernier tour enveloppant tous les autres; cloisons unies; dos caréné.

\section{Espèce servant de type au genre.}

\section{L'Ilote rotalé. Ilotes rotalitatus.}

Nautilus orbiculus. En allemand, der scheibenformige schiffer. Testac. microsc. a Leo. von Fichtel, etc., pag. 112. tab. 21 . fig. a-d.

Cette coquille forme aussi un des anneaux de la grande chaine que nous venons d'indiquer. Indépendamment de sa cellulation, sa bouche est encore trèsremarquable par l'échancrure triangulaire qu'elle présente sur le bord de sa carène; sa spire est excentrique, ce qui la distingue particulièrement des numulies et de quelques genres qui en sont voisins, et les sommets de cette spire sont mamelonnés sur les deux flancs, ce qui 
constitue un nouvel ordre de gentc. D'ailleurs les ilotes out un retour de spire dans la maniere des nautiles; la bouche en s'avançant graduellement clargit le tèt ou coquille, et lilote seroit pour nous un phoneme, si cette bouche n'étoit pas cellulée. Sa carine est assez tranchante, parce que l'ouverture de la coquille se dessine en ogive très-alongé. Indépendamment des traits ou cótes demi-circulaires qui partent du centre pour se rendre à la circonférence, et qui sont formés par les cloisons, lilote rotalé nous montre encore des stries fines, transversales, et tournées dans le sens de la spire : elles indiquent les cellules.

On trouve cet ilote à Livourne et sur les plages sablonneuses de la Méditerranée; en général ce n’est que dans les sables que se tiennent des coquilles flottantes et fragiles, qui, balottées par les flots, ne pourroient que se briser contre les rochers, à moins que, semblables aux mollusques rampans et testacés, leurs animaux ne vinssent s'y attacher.

La couleur de lilote rotalé est blanche, teintée de violet, et reflétée en or ; la nacre en est extrêmement fine, mais très-irisée, quoique perlucide.

11 a presque une ligne de diametre. 
Coquille en disque, mamelonnée, contournée en spirale. 


\section{LI* GENRE.}

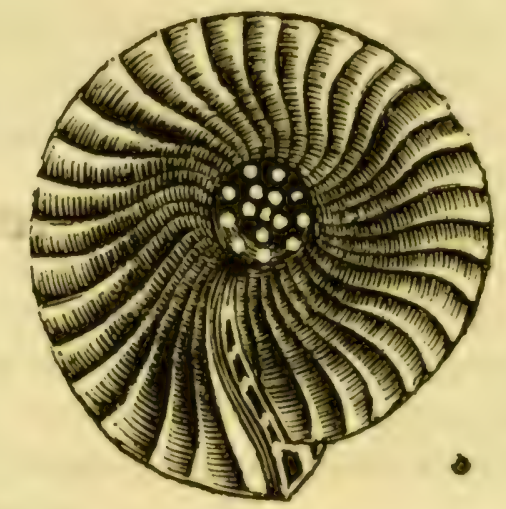

THEMLONE; en latin, Tusmeon. 


\section{LE THÉMÉONE.}

Caractères génériques. Coquille libre, univalve, cloisonnée et cellulée; contournée er spirale, et formée en disque conique aplati sur ses deux flancs ; mamelonnée sur les deux centres; le dernier tour de spire renfermant tous les autres; dos aigu; bouche en ogive, cellulée et recevant dans son milieu le retour de la spire; cloisons unies.

Espèce servant de type au genre.

Le Théméone frisé. Themeon rigatus.

Nautilus crispus; en allemand, der krause schiffer. Testac. microsc. a Leo. von Fichtel, etc., pag. 40. tab. 4. fig. d. e. f. et tab. 5. fig. a. b. Der korner nautilus. Stat. Muller. Das punktirte und mit reifen umlegte ammonshorn. Schrœter. Das punktirte ammonshorn. Schreibers. Nautilus striatus vulgatissimus. Soldani.

Linn. Syst. nat. edit. XII. tom. I. pag. r 162. sp. 265.

- Natursystem. V. Stat. Muller. Th. 6. Bd. I. pag. 356. $\mathrm{Sp} .275$.

- Syst. nov. ed. XIII. Gmel. T. I. P. 6. p. 3370. sp. 3.

Planc. conch. pag. 10. tab. I. fig. 2. Très-mauvaise. Gualt. ind. testac. tab. rg. fig. A. D. Mauvaise. Ginanni ad tab. I4. f. I I 2 .

Ledermuller mic. tab. 8. fig. B. mauvaise. Martini conch. cab. Bd. 3. p. 248-253 et tab. 20 f. 172,175 . 
(de Gualt.) et 174 (de Lederm.) Schroeter conch. kemut. B. I. p. ro.sp. 3. Schreibers couch. kennt. B. r. p. 5. sp. 5. Soldani sag. orit. p. roo. tab. 2 lig. r 7. Y. Z. ćl le méme testac. T. I. P. I. p. 54. tab. 55. f. F. et tab. $3 /$. G. H.

Le Théméone frisé présente un disque relevé sur les deux flancs en cóne aplati, dont la pointe des deux côtús est surmontée par un mamelon criblé de pores: ils recouvrent le noyau ou centre de la coquille, qui est assez solide, et dessinée en orbe comme les numulies; ellerenferme de même intérieurement des cellules nombreuses, mais ici elles sont indiquées par la bouche cellulée et crénelée ; et quoique Gualtieri nous ait scml)lé vouloir indiquer un siphon dans la figure peu exacte qu'il nous a domée de cette coquille, nous croyons cependant, conduits par l'analogie, que sil existe des siphons dans ces coquilles, on doit les chercher dans le fond de chaque cellule ou concamération, dans la supposition ou nous sommes que chacune d'elles est liabitie par un animal isolé, mais point solitaire, attendu qu'il fait partic d'une assez nombreuse famille, co-habitante sous un toit commun aux cellules de chacun de ses membles.

Le théméone frisé vit dans la Méditerranée; on le trouve encore dans celle Adriatique, et on le rencontre fossile dans beaucoup d'endroits de la Toscane.

A l'état vivant ce théméone est d'une couleur blanche, perlée et irisée; les mamelons sont bleuaitres : à l'état fossile, il partage la teinte des bancs ou il git.

Il a une demi-ligne dans son plus grand diametre; tranché sur le plus petit, lą coupe présente un losangge. 
Coquille en disque, mamelonnée, et contournée en spirale. 


\section{LII* GENRE.}

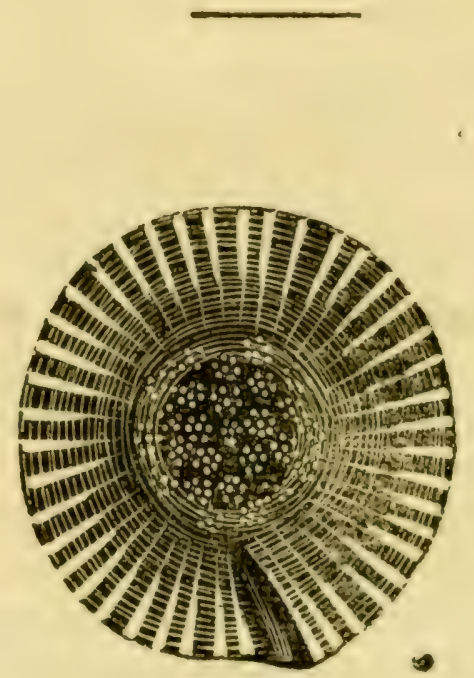

CEllulie; en latin, Celiatrues. 


\section{LE CELLULIE.}

Caractères génériques. Coquille libre, univalve, cloisonée, cellulée, en disque aplati, et contournée en spirale; mamelonnée sur les deux centres; le dernier tour de spire renfermant tous les autres; dos obtus mais caréné; bouche en ogive écrasé contre le retour de la spire qu'elle recoit dans son milieu, à demi-recouverte par un diaphragme; cloisons unies.

Espèce servant de type au genre.

Le Cellulie grilloné. Cellanthus craticulatus.

Nautilus craticulatus. Der geflochtene schiffer. Testac. microsc. a Leo. von Fichtel, etc., pag. 5 r. tab. 5. fig. h. i. k.

Parmi les genres que nous parcourons actuellement, on verra dans chacun d'eux les mamelons des deux flancs placés d'une manière centrale; ce sont ces mamelons très-caractéristiques qui nous ont surtout engagé à former ces genres. Jusqu'à présent, on a dû voir les mêmes formes se répéter et reparoître pour ainsi dire successivement, mais avec des modifications tranchantes. C'est ainsi qu'après avoir passé en revue les nautiles ct leurs genres voisins, mais tous sans ombilic, nous avons traité des océanies, autrefois nautiles ombiliqués, et qui en nous fournissant les océanies, les pélaguses, 
les mílonies, les antenores, nous donnirent quatre genres tres-rapprochés, chamons qui nous conduisirent aux ammonies. Celles-ci nous présenterent tous leurs tours de spire à découvert, et c'est ainsi qu'en passant au travers d'une foule de modes d'être de la même matiere, traitant tantôt des coquilles multiloculaires turbinées et non-turbinées, ombiliqures sur um plan, aplaties, à spires recouvertes, nous sommes arriris a celles mamelonnées, qui de méme offient une foule de modifications, au milieu desquelles nous avons cherché à étałlir celles propres à former les types de leurs genres. Les mamelons des cellulies grillonnés, extrêmement saillans, occupent au moins le tiers du diametre de la coquille, qui est comme craticuléc ou grillée ; ces mamelons sont chargés de petits pores disposés en étoiles, et ressemblant à autant de fleurs; cette coquille est fortement renflée, et elle seroit semi-globuleuse, sans sa carime obtuse. La bouche en ogive écrasé, n'est recouverte qu'en partie par son diaphragme, qu'on peut envisager comme formé en faux.

La couleur de ce cellulie est blanche, tcintée d'azur et légirement nacrée; teinte et iris que partagent les mamelons. Ces coquilles se trouvent dans les sables des bords du golfe d'Arabie, ou Spengler les rencontra le premier, mais jetées sur la plage par les flots, et nayant plus leurs animaux.

Lecellulie grilloné a une demi-ligne de diamètre sur son plan. 
Coquille en disque, mamelonnée ; et contournée en spirale. 
COQUILI, ES

\section{LIII: GENRE.}

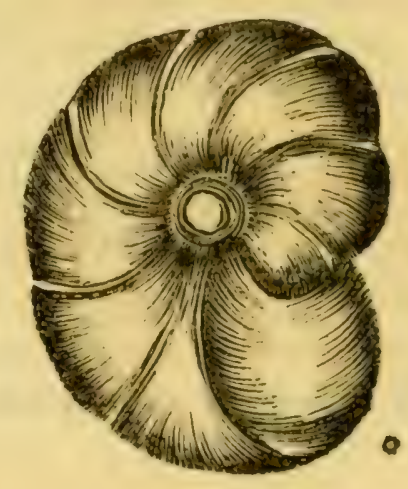

NONIONE; en latin, Noxion. 


\section{LE NONIONE.}

Caractères génériques. Coquille libre, univalve, cloisonnée, en disque, et contournée en spirale; mamelonnée sur les deux centres, le dernier tour de spire renfermant tous les autres; dos renflé, bouche arrondie, recouverte par un diaphragme ouvert en croissant contre le retour de la spire, qu'elle reçoit dans son milieu; cloisons unies.

\section{Espèce servant de type au genre.}

\section{Le Nonion soufflé. Nonion incrassatus.}

Nautilus incrassatus. Der aufgeblasene schiffer. Testac. microsc. a Leo. von Fichtel, etc. pag. 38. tab. 4. fig. a, b, c.

Nous retrouvons, au milieu des coquilles mamelonnées, les formes des nautiles: celles-ci sont simplement cloisonnées et non-cellulées. Peut-être nous fera-t-on un reproche de n'avoir point serré les uns contre les autres, les genres qui se rapprochoient le plus par leurs formes extérieures ; mais nous avons cru devoir, peutêtre à tort, en agir différemment ; les formes en général nous ont peu arrèté, mais nous avons pesé davantage sur la bouche, sur la spire, sur l'ombilic, sur son absence, et sur des cals ou mamelons. Un système, comme nous l'avons dit dans notre discours préliminaire, est une chose de convention : il est un moyen de se retrou- 
ver, de se recomnoitre au milieu d'une foule d'oljojets; il les classe diune maniere précise, mais plus ou moins arbitraire. Convaincu de cette vérité, nous avons tellement disposé la partie typographique de notre ouvrage, qu'on peut le démembrer à volonté; afin que chaque conchyliologue puisse varier, d'aprés ses desirs ou ses vues, l'ordie que nous avons suivi, mais que nous sommes bien loin de prétendre avoir institué à demeure.

Nous avons sommis à plusieurs reprises le nonion soufflé au microscope, et dans ces observations les naturalistes sarent combien il faut se metre en garde contre l'illusion de l'optique et le brisement des rayons lumineux; cependant ce nonione nous a paru constamment recouvert d'une myriade de pores, assez profonds à la vérité, mais tellement petits, quïl est impossible de les rendre par le dessin. La bouche est très-ćvasée, le diapluragme bombé, et l'ouverture semi-lunaire trés-apparente contre le retour de la sjire: les cloisons sont de mème très-visibles, assez nombreuses, et les concamérations sont renflées circulairement.

Ce nonione est blanc, teinté de rose. On le trouve dans les sables sur le bord de la mer à Porto-Ferrajo, à lile d'Elbe, dansla Méditerrancéc; il a une denu-ligne de cliamètre. 
Coquille en disque, mamelonnée; et contournée en spirale. 


\section{LIVe. GENRE.}

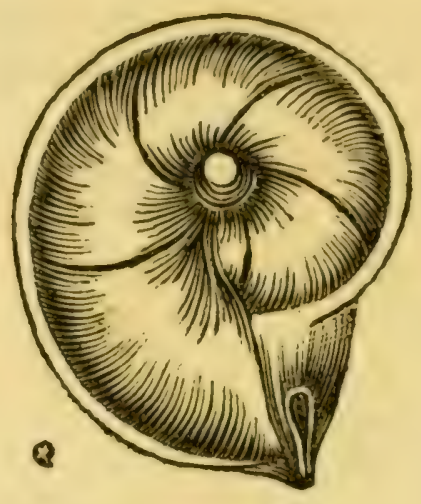

ROBULE; en latin, Rosulus. 


\section{LE ROB ULE.}

Caractères génériques. Coquille libre, univalve, cloisonnée, en disque, et contournée en spirale, mamelonnée sur les deux centres; le dernier tour de spire renfermant tous les autres; dos caréné et armé ; bouche triangulaire, recouverte par un diaphragme, et recevant dans son milieu le retour de la spire, percée à l'angle extérieur d'une rimule piriforme; cloisons unies.

\section{Espèce servant de type au genre.}

\section{Le Robule tranchant. Robulus cultratus.}

Nautilus calcar. Der spornschiffer. Testac. microsc. a Leo. von Fichtel, etc., onzième variété. pag. $7^{8}$. tab. I5. fig. c, f, g.

Offrant de nouveau à l'extérieur presque les mêmes formes que les nautiles et les angulithes, le robule tranchant est encore caréné ; mais indépendamment de cette carène tranchante, ce genre est d'autant plus singulier, qu'au lieu du siphon il montre à l'angle extérieur une rimule, une fente plissée, un spincter enfin fait en cul de poule, et dessiné en poire; la coquille qui nous sert de type présente de plus des cloisons très-éloignées les unesdes autres, et successivement en couvrant la bouche, elles servent de diaphragme.

Il est une observation générale, et qui paroîz appar- 
tenir a la tolalité des coquilles cloisonnées et cellulées; jamais elles ne sont recouvertes de mousse ou de drap marin, et toujours elles sont lisses et polies; jusqüa présent nous n'avons pas encore trouvé d'exception à cette rigle, et nous croyons pouvoir en infirer que les mollusques qui les construisent les embrassent en dehors par leur manteau en les rendant lisses et irisées, polies et nacrées: non pas qu'elles soient des coquilles intérieures ou renfermées dans le corps d'un mollusque, comme nous le verrons pour quelques autres genres, mais les coquilles qui constituent ceux dont nous par. lons sont entierement recouvertes par un manteau que leurs mollusques ouvrent à volonté.

Dans le robule tranchant les cloisons marquent autant de côtes, et quoique la coquille soit unie, ces côtes sont tres-apparentes; elle est de plus très-renflée; et ses mamelons sont unis, mais saillans : ils sont blancs et teintés d'orangé, comme la coquille.

On trouve le robule tranchant à la Coroncinc, en Toscane.

Il a trois quarts de ligne de diamitre. 
Coquille en disque, mamelonnée ; et contournée en spirale. 


\section{LVe. GENRE.}

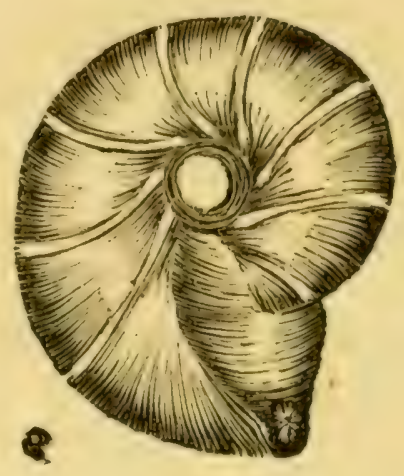

PATrocle; cn latin, Patrocles. 


\section{LE PATROCLE.}

Caractères génériques. Coquille libre, univalve, cloisonnée, en disque, et contournée en spirale ; mamelonnée sur les deux centres; le dernier tour de spire renfermant tous les autres; dos obtus mais caréné; bouche triangulaire, piriforme, recouverte par un diaphragne qui reçoit dans son milieu le retour de la spire, percée à l'angle extérieur par une rimule étoilée et ovale; cloisons unies.

Espèce servant de type au genre.

Patrocle dissident. Patrocles querelans.

Nautilus calcar. Der spornschiffer. Testac. microsc. a Leo. von Fichtel, etc. , pag. 76. tab. I 2. fig. g, h. septième variété.

Jani Planci de conch. min. not. pag. I2. tab. I. fig. 3. très-médiocre.

Ledermullcr, microsc. gem. u. aug. ergœtz. tab. 8 . fig. c, médiocre.

Martini, conch. cab. I. tab. Ig. f. I7I. copiée de Ledermuller.

Rien ne prouve autant le besoin de ranger enfin dans un ordre systématique, et sous des dénominations génériques, mais à coupes un peu resserrées, les coquilles 
microscopirjues; coquilles si négligées jusqu’à nos jours, ct yui cependant jonent un si grand rôle dans la texture du globe terraqué. Nous voyons ici, malgré l'exactitude de von Fichtel et de von Moll, ranger peut-étre pour la vingtieme fois parmi les nauliles, et, ce qui est bien plus fort, parmi les nautiles armés ct ćperonnés, une coquille dont la carine est obtuse, lisse, unic, et sans armure; et cependant clle n'est pas un nautile, tant par ses deux mamelons, que par sa bouche recouverte et percée à l'angle extéricur par une rimule étoilée qui remplace le siphion. Il pảroît que cette espece a échappé ou a été inconnue à Soldani: ses cloisons sout trés-apparentes, elles prennent la direction inverse au sens de la volutation de la coquille, étant arquées en arrière; ses mamelons sont lisses.

On trouve le patrocle dissident à l'état marin, et de coulcur blanchâtre rosacée, dans les mers Adriatique et Méditerranéc. On le rencontre aussi fossile en grande abondance dans les bancs de la Coroncine, jrès de Sicune, en Toscane, et alors sa teinte ferruginée tire sur le roux.

Del'une ou de l'autre maniere on en voit qui ont juscqu’à une ligne et demic de diamètre, et même jusqu’à deux lignes. 
Coquille en disque, mamelonnée, et contournée en spirale. 
LVI $\mathrm{I}^{e}$ GENRE.

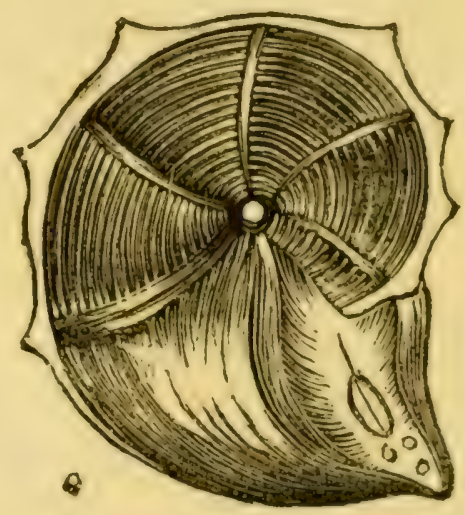

SPINCTERULE; en latin, SPigctFrLes. 


\section{LE SPINCTÉRULE.}

Caractères génériques. Coquille libre, univalve, cloisonnée, en disque, et contournée en spirale ; mamelonnée sur les deux centres; le dernier tour de spire renfermant tous les autres; dos arrondi, caréné, armé, bouche évasée, recouverte par un diaphragme percéde trois trous, disposés en triangle à l'angle extérieur, ainsi que d'une rimule au centre, dont la fente se prolonge vers le retour de la spire qui est reçu dans le milieu du diaphragme.

\section{Espèce servant de type au genre.}

Le Spinctérule membré. Spincterules costatus.

Nautilus costatus. Der geribbte schiffer. Testac. microsc. a Leo. von Fichtel, etc. , pag. 47. tab. 4. f, g, $\mathrm{h}, \mathrm{i}$.

Indépendamment de ses côtes saillantes, formées par les cloisons et arquées en sens inverse de l'accroissement, ce spinctérule est encore finement strié dans le sens de sa circonférence. Parmi ces côtes il en est de plus épaisses les unes que les autres, et il en est même qui paroissent 
triplées ou redoublées dans leur épaisseur. Les deux mamelons sont petits, mais saillans, et toute la coquille est renflée dans la manière des nautiles.

La bouche de cette coquille est une des plus remarquables de toutes celles que nous ayons encore décrites, et que nous ayons rencontrées parmi les coquilles microscopiques, en raison de ses stigmates ou siphons, et de sa rimule longuement fendue; son diaphragme est aussi relevé en dos d'âne.

La couleur est blanche, teintée de vert d'émeraude, les mamelons sont roses.

On trouve le spinctérule membré en très-grande abondance en Afrique, sur la còte du royaume de Maroc; il a une demi-ligne de diamétre. 
Coquille en disque, mamelonnée, et contournée en spirale. 
LVII: GENRE.

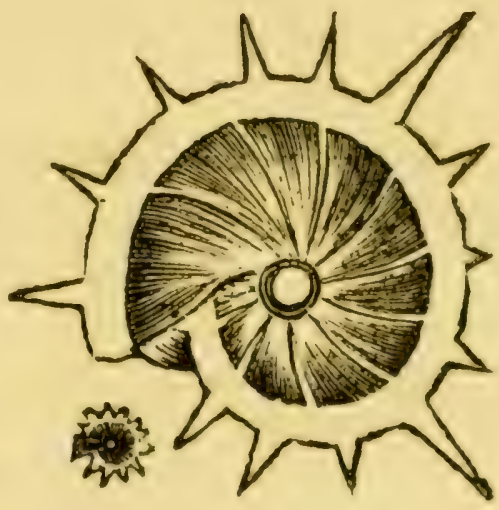

CLISIPHONTE ; en latin ChIŚIPHontres: 


\section{LE CLISIPHONTE.}

Caractères génériques. Coquille libre, univalve, cloisonnée, en disque, et contournée en spirale; mamelonnée sur les deux centres, le dernier tour de spire renfermant tous les autres; dos caréné et armé; bouche triangulaire, ouverte, recevant dans son milieu le retour de la spire; cloisons unies, percées par un siphon.

\section{Espece servant de type au genre.}

\section{Le Clisiphonte molette. Clisiphontes calcar.}

Hist. gen. et part. des Miollusq. Buffon. édit. de Sonnini, tom. 4. pag. 219. pl. XLVII. fig. 4. Tirée de la Testacéographie de Suldani.

Cette jolie coquille qui rentre encore dans les formes des angulithes, à l'exception de ses deux mamelons, est armée tout autour de sa carène en molettes d'éperon ;ses cloisons sont très-apparentes, et elles tranchent au travers de sa robe iriscee et diaphane.

Elle est mince, d'une couleur azurée, et ses cloisons marquent en brun.

Le clisiphonte molette vient de l'Océan indien; on le trouve en très-grande quantité sur les rivages des îles de 
Bornén et de Java, oi nous l'avons reconnu: quant a celui que publia Soldani, eł que nous dessinâmes d'après lui, dans l'édition de Buffon que nous venons de citer, il venoit de la Méditerranće; cependant Soldxni qui en avoit réuni quekyues milliers, leur assigna un tiers de ligne dans leur plus grand diametre.

Le clisiphonte molette de l'Océan indien a un peu moins de six lignes, d'une pointe de son armure à une autre. 
Coquille en disque, mametonnée, et contournée en spirale. 


\section{GENRE.}

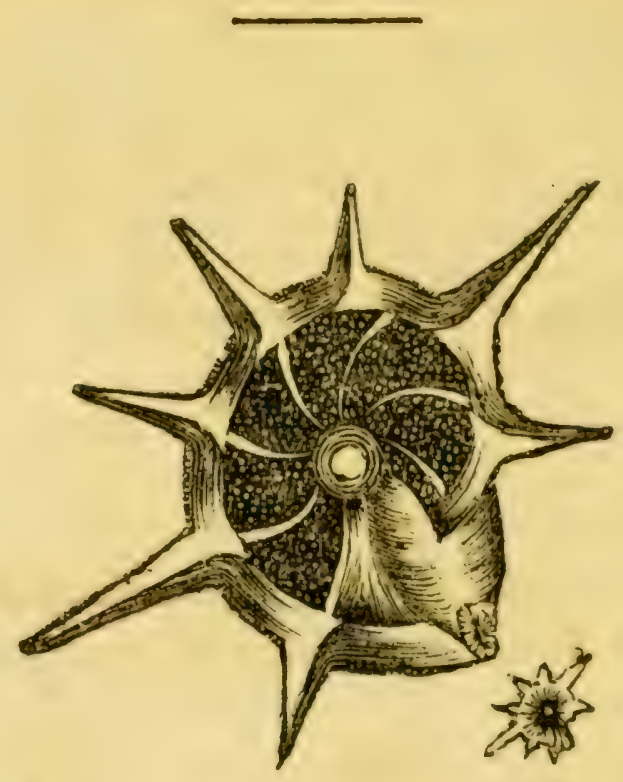

HÉRIONE; en latin Henox. 


\section{L'HERIONE.}

Caractères gennériques. Coquille libre, univalve, cloisonnée, en disque, et contournée en spiralc ; mamelonnée sur ses deux centres ; le dernier tour de spire renfermant tous les autres; dos caréné et armé ; bouche triangulaire, piriforme, recouverte par un diaphragme percé à l'angle extérieur par une fissure en rimule étoilée, et recevant dans son milieu le retour de la spire; cloisons unies.

\section{Espèce servant de type au genre.}

\section{L'Hérione rostré. Herion rostratus.}

Nautilus calcar. Derspornschiffer. Testac. microsc. a Leo. von Fichtel, etc. , pag. 74. tab. 12. fig. a , b , c , cinquième variété.

L'hérion rostré sort de l'ordre des coquilles microscopiques, observation que déjà nous eussions pu faire pour quelques autres, ou au moins ce sont les plus grandes coquilles sablonneuses. Celle que nous décrivons est grainée dans toute sa superficie, à l'exception de la dernière concamération qui est lisse; elle porte le diaphrazme de la bouche, qui lui-même est uni : les cloisons sont arquées en sens inverse, très-apparentes, e assez éloignées les unes des autres; lisses et diaphane; comme la glace la plus pure, les mamelons ont une 
tcinte couleur de rose; et le dos est non-seulement carúné, mais celte carene est dentelie en scie, et porte de distance en distance des espéces de pointes ou d'épines en forme de rostres ou d'éperons de galéres, dont les uncs sont plus longues que les autres, et qui sont au nombre de sept à huit.

On peut regarder lhérione rostré comme très-rare, à moins que, comme les épines ou pointes sont très-fragiles, elles ne soient abjattues dans le plus grand nombre d'individus; et alors il seroit possible de retrouver cettr. coruille daus celle décrite el figurée par Soldani (saggin orittografico, pag. (,8.tab. r. fig. 6. J.) et dans la Testaccingraphie du même auteur, T. I. P. I. tab. 59. fig. qq. zz.

Indépendamment du mamelon rose, sa couleur est blanche, flambée et perlée dans l'état marin. Lorsque la coquille est fossile, elle prend une teinte ferrugineuse ct rongeâtre, et quelquefois mème une couleur envinće.

On trouve l'hérione rostré dans la mer Adriatique, et surtont sur le rivage de Rimini: quant au fossile, on le recucille à la Coroncine, prés de Sienne en Toscane.

D'une pointe à l'autre cet hérione a près de six lignes de diamitre; la coquille, proprement dite, en a trois et demi. 
Coquille en disque, mamelonnée, et contournée en spirale. 


\section{LIX: GENRE.}

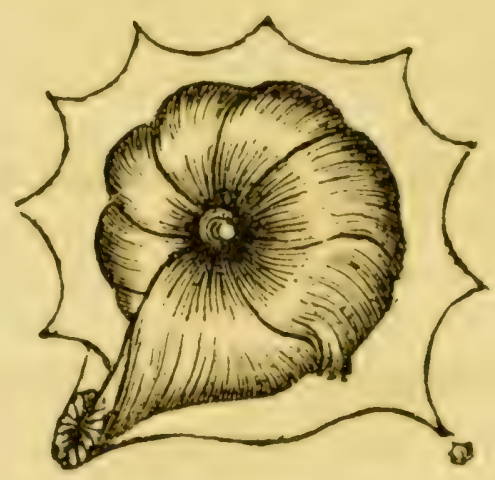

RHINOCURE; en latin, Rmrocurus. 


\section{LE RHINOCURE.}

Caractères génériques. Coquille libre, univalve, cloisonnée, en disque, et contournée en spirale; mamelonnée sur ses deux centres; le dernier tour de spire renfermant tous les autres; dos caréné et armé ; bouche oblongue, arrondie, recouverte par un diaphragme qui porte à son extrémité extérieure une rimule ovale, plissée en forme de spincter, fendue dans sa longueur, cette fente se prolongeant jusqu'au retour de spire, qui est reçu dans le milieu du diaphragme; cloisons unies.

\section{Espèce servant de type au genre.}

Le Rhinocure aranéeux. Rhinocurus araneosus.

Soldani, testac. tab. 58. v. 191. hh.

Cette jolie coquille qui vient se ranger parmi celles qui sont éperonnées, est d'un tissu si délicat, qu'elle semble le disputer de finesse aux toiles d'araignées; elle est mamelonnée sur ses deux flancs comme celles qui précèdent; et sa bouche aussi singulière que nouvelle, quant aux observations conchyliologiques, va nous conduire à des bouches plus compliquées encore: ses cloisons sont arquées d'une manière molle et sinueuse, et le dos du rhinocure aranéeux est armé médiatement d'une lame testacée qui se dessine en festons. 
Diaphane et presque limpide, cette coquille offre toutes les couleurs, les nuances, les teintes et les reflets de l'iris; on peut chercher à rendre ces teintes, mais y parvenir seroit difficile.

On trouve le rhinocure aranéeux sur les plages de la mer Adriatique; et fossile, mais de couleur ocracée, à la Coroncine.

Il a plus d'unc ligne de diametre. 
Coquille en disque, mamelonnée, et contournée en spirale. 
GOCUILI. E 5

\section{LXe. GENRE。}

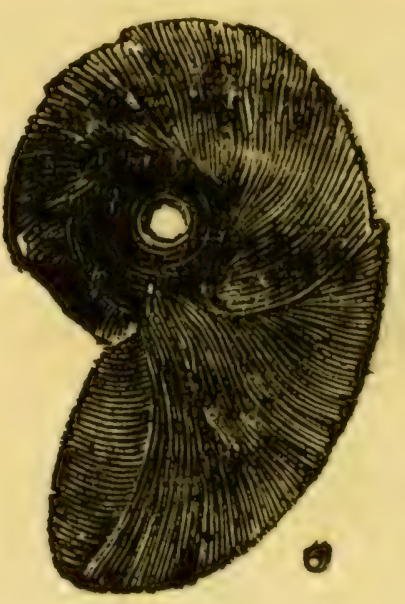

MACRODITE; en latin, MAcrodites. 


\section{LE MACRODITE.}

Caractères génériques. Coquille libre, univalve, cloisonnée, en disque, et contournée en spirale ; mamelonnée sur ses deux centres; le dernier tour de spire renfermant tous les autres; dos arrondi; bouche oblongue, recouverte par un diaphragme; siphon inconnu; cloisons unies.

\section{Espèce servant de type au genre.}

\section{Macrodite cuculé. Macrodites cucullatus.}

D'après ses formes allongées, on pourroit ranger cette coquille parmi celles elliptiques, avec qui elle a beaucoup d'analogie : les mamelons sont très -grands, de forme orbiculaire, et c'est autour d'eux que viennent s'enrouler les concamérations, qui toutes forme nle capuchon, et dont les cloisons sont arquées dans le sens opposé à la croissance de la coquille; chacune de ces concanérations est renflée en particulier, et elles enjambent successivement sur l'orbe des mamelons. La bouche nous a paru totalement fermée, et son diaphragme nous a semblé n'avoir point de solution de continuité ; mais nous croyons qu'il manque à cet égard quelque chose à nos recherches. D'autres observateurs seront plus heureux, et nous ne doutons point quills ne trouvent un siphon, une rimule, une fente que nous avons cherchés en vain. 
Le macrodite cuculé est teinté de ronge, de jaune, de bleu, et il reflete dans sa nacre pellucide toutes les nuances intermédiaires du spectre solaire; on ne sauroit mieux le comparer qu'à une bulle de savon, tant il est diaphane.

On le trouve sur les rivages de l'Adriatique, ou il est jetẻ par les flots.

Il a une ligne as demie de longucur. 
Coquille elliptique, mamelonnée ; et contournée en spirale. 


\section{LXI॰. GENRE.}

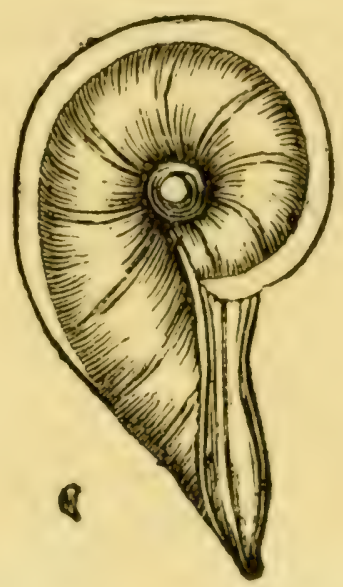

LAMP',IDIF, ; en latin , LAMras. 


\section{LE LAMPADIE.}

Caractères génériques. Coquille libre, univalve, cloisonnee, en disque et elliptique, contournée en spirale, mamelonnée sur les deax centres; le dernier tour de spira renfermant tous les autres; dos caréné et armé; bouche lancéolée, terminée en tubercule, couverte par un diaphragme fendu dans toute sa longueur, et rece vant dans son milieu le retour de la spire; cloisons unies.

\section{Espèce servant de type au genre.}

Le Lampadie trithème. Lampas trithemus.

Nautilus calcar. Der spornschiffer. Testac. a Leo. von Fichtel, etc. , pag. 75. tab. 12. fig. d, e, f. sixième variété.

Soldani, testac. T. I. P. I. tab. 58. C. gg. hh. ii. kk. mm. et tab. 59. fig. qq. pag. 64. Nautiles carinatos. Figures assez mauvaises.

Les coquilles de ce genre se dessinent en forme de lampe; celle qui en présente le type est assez renflée, et ses mamelons sont très-proéminens; le bec de l'angle extérieur de la bouche est fait en cul de poule, et il offre une espèce de tubercule; la carène en est obtuse, mais elle porte une armature unie et vitrée; le têt est de même très-lisse ; quant à ses cloisons elles sont très-apparentes, 
et on les voit trés-distinctement au travers ; leur direction est arquée en sens contraire de l'accroissement de la coquille; elles y forment comme autant de côtes plus fortement colorées.

La couleur du lampadie trithème est brunâtre et ocracíe, parce que jusquà présent on ne l'a rencontré que dans l'état fossile, dans des terres et dans des bancs ferrugineux, et qu'il partage la couleur locale de son gisenaent; on l'a principalement trouvé à Ripalta près Saint-Quirico, dans le voisinage de Sienne en Toscane, ou d'anciennes révolutions maritimes l'ont déposé en masses très-considérables, avec d'autres coquilles devenues comme lui fossiles.

Paryenu à tout son accroissement, ce lampadie a quelquefois une ligne et demie de longueur. 
Coquille elliptique, mamelonnée; enretour sur elle-même. 


\section{LXII* GENRE.}

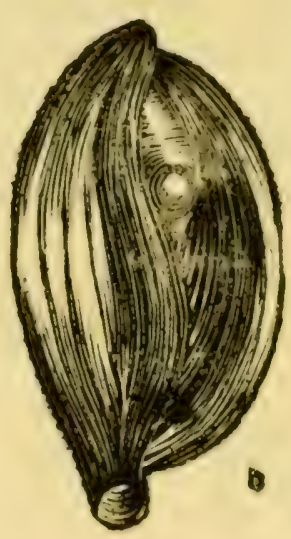

POLlONTE; en latin, Poleovtes. 


\section{LE POLLONTE.}

Caractères génériques. Coquille libre, univalve, cloisonnée, en retour sur elle-même, mais en disque; elliptique; mamelonnée sur ses deux centres; dos arrondi ; bouche ouverte, ronde, dégagée, portée sur un col, et terminant une chambre faite en forme de cosse ou de sac; cloisons unies, en calotte; siphon inconnu.

\section{Espèce servant de type au genre.}

Le Pollonte vésiculaire. Pollontes vesicularis.

Polythalame de Soldani. Testac. tab. 154. cc.?

La structure singuliere de cette coquille pourroit nous faire croire que son nollusque forme chaque année une cosse ou gaine nouvelle, latérale à l'ancienne, et en retour sur un noyau primitif, dessiné par les mamelcns; mamelons qui la rapprochent des genres qui préciedent. Ces gaines ou sacs ont beaucoup de ressemblance avec les vésicules aériennes de certains poissons, tels que la carpe, le brochet, etc.; mais ils sont terminés par une bouchc arrondie, portée sur une espèce de collet ou étranglement, le sac ou concamération étant renflé dans son milieu, et devenant plus étroit aux deux bouts dont celui postérieur est terminé en pointe très-émoussée; et quoique la spire de cette coquille ne puisse point être regardée comme parfaitement régulière, il n'en est 
pas moins vaii (pu'elle ne doit point ètre rangée parmi les coquilles droites, auxquelles cependant elle commence it nous conduire, faisant un de ces chainons intermódiaires, qui tienuent aux genres qui précédent, a ceux qui viennent les suive, en nous conduisant sans ressaut à d'autres modifications assez majeures pour exiger une classification ou aumoins une dénomination générale.

Le pollonte vésiculaire est orangé et irisé. On le trouve sur les plages qui bordent l'Océan indien, et sur celles de la Méditerranée. Il a presque une ligne de longueur.

C'est ici que nous allons quitter les coquilles cloisonnées et contournées en spirale, pour entrer dans un nourel ordre de choses; désormais nous n'aurons plus dans les têts cloisonnés que des coquilles droites, ou au moins dont le seul bout sera arqué et roulé, tandis que le reste nous présentera une espèce de fût, et c'est ainsi que sans violence, nous atteindrons les belemnites tes genres qui en sont voisins. Aprés ces coquilles droites, nous en vermons quelques-unes de torses, et ce sera par clles que nous terminerons le premier volume de notre conchy-liologie, uniquement consacré aux coquilles cloisonnées. 


\title{
COQUILLES
}

UNIVALVES CLOISONNÉES,

\author{
DROITES.
}

Coquille droite, sommet spiré. 


\section{GENRE。}

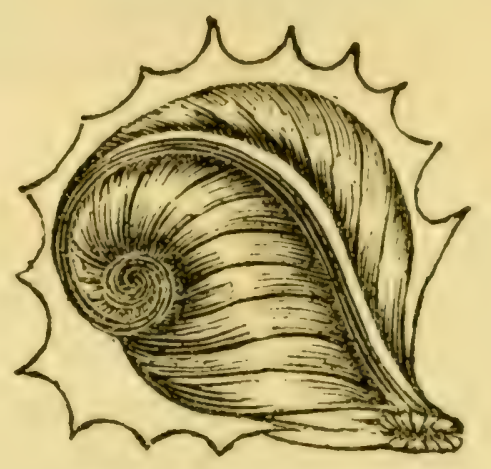

SCORTIME; en latin, Sconrrarcs. 


\section{LE SCORTIME.}

Caractères génériques. Coquille libre, univalve, cloisonnée ; droite, à sommet spiré, ayant une veine carénée sur ses deux flancs; dos arrondi, caréné et armé en molette d'éperon; bouche allongée, recouverte par un diaphragme fendu dans sa longueur, et terminée par un siphon figuré en sphincter ou bourse; cloisons unies.

\section{Espèce servant de type au genre.}

Le Scortime naviculaire. Scortimus navicularis.

Polythalame de Soldani. Testac. tab. 55 . vas. 187, D.

Dans le plan que nous nous sommes tracés, il nous seroit difficile d'indiquer des ressauts et des séparations brusques et tranchées, séparations que l'ordre naturel semble fuir constamment, parce que chez lui tout est fondu, tout se lie, el que les êtres se rattachent l'un à l'autre d'une manière insensible. Nous avouons que cette façon d'envisager les choses, est peut-être celle qui nous a donné le plus de travail, mais aussi nous nous flattons d'être arrivé à notre but jusqu'à un certain point, d'autant plus que nos grandes divisions se fondent dans leurs genres respectifs, et que ceux-ci se nuancent tellement dans leurs espices, que nous regardons le point de 
séparation comme impossible à indiruer; et c'est ce que nous pourrons prouver un jour, en publiant ces mémes especes: travail immense, mais dont nous nous occupous. 'Telle est la marche die la nature, qui a revêtu toutes les formes, et qui n'a laissé aucume place vacante.

Le scortime naviculaire, que nous regardons comme une corpuille droite, ou au moins mitoyenne, présente une organisation singuliere par la veine, varice, ou còte qui carène ses flancs, en divisant ses concamérations en deux séries distinctes; une carène éperonnée et dorsale, ainsi qu'une bouche terminée par un spincter, rapprochent cependant encore cette coquille de quelques-unes d'enire celles que nous venons de parcourir.

Sa couleur est bleuâtre, irisée en vert et reflétée de jaune; toutes ses cloisons sont apparentes, et la spire n'est plus recue dans la bouche, ni roulée contre elle.

Ce scortime vient des Canaries, on le trouve encore dans la Méditerrané; il acquiert quelynefois la dinension de l'ongle du petit doigt. 
Coquille droite, sommet spiré. 


\section{LXIV*. GENRE.}

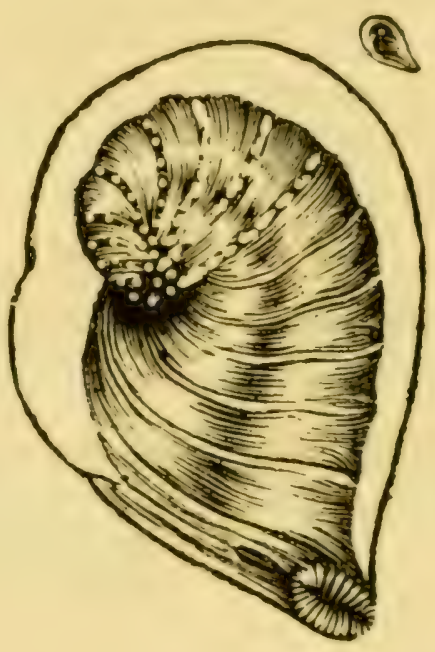

LINTHURIE; enlatin, Listucnis. 


\section{LE LINTHURIE.}

Caractères génériaues. Coquille libre, univalve, cloisonnée, droite, à sımmet spiré; aplatie; dos arrondi , mais armé; bouche allongée, recouverte d'un diaphragre fendu dans toute sa longueur, terminé au bout extérieur par un siphon en spincter, précédé par un eufoncement en fer de lance; cloisons unies.

\section{Espèce servant de type au genre.}

Le Linthurie casqué. Linthuris cassidatus.

Nautilus cassis. Der helmformige schiffer. Testac. microsc. a Leo. von Fichtel, etc., pag. 97. var. 2. tab. I7. fig. e, g.

Soldani, saggio oritt. pag. 97. tab. I. f. 1. A. B. C.

D'après ses formes extérieures on peut regarder cette coquille comme ayant deux aspects différens, l'un appartenant au jeune âge, et l'autre à celui plus avancé. Lorsqu'elle est jeune, l'emplacement de ses cloisons se marque sur le têt par des cordons perlés, ce qui n'a point lieu plus tard, et alors ces côtes sont unies, quoique toujours très-apparentes. La bouche du linthurie casqué est d'autant plus caractéristique que son spincter ou siphon est précédé par un enfoncement en fer de lance; le dos arrondi est garni par une lame ou crête transparente, vitrée et diaphane; et par son ensemble cette coquille ressemble assezà un casque grec. Les côtes 
indicatives des cluisons sont doubles et assez larges; elles se courbent dans le sens de l'accroissement de la coquille, qui, entre elles, est finement striée; et il est probable que le clangenent de forme qu'elle éprouve avec l'âse, a dú plus d'une fois induire en erreur les observateurs qui auront cru y reconnoitre deux coquilles différentes.

Le linthurie casque est d'un blanc jaunâtre, mais argentin, sur lequel tranchent des perlures et des côtes orangées; plussouvent encore il est entièrement ocracé, parce que jusqu’à présent on ne l'a rencontré qu'à l'état fossile à la Coroncine prìs de Siemne, en 'Tuscane.

Cette jolie coquille est de la grandeur d'une lentille ordinaire. 
Coquille droite, sommet spiré. 


\section{GENRE。}

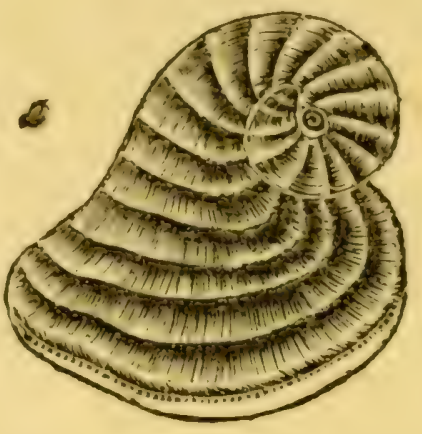

PÉxĺrople; en latin, Pexerorlis. 


\section{LE PÉ NÉROPLE.}

Caractères génériques. Coquille libre, univalve, cloisonnée, cellulée, droite, et à sommet spiré, formée en corne d'abondance aplatie; bouche de toute la longueur de la base, et percée sérialement par une file de pores; dos arrondi; cloisons unies.

\section{Espèce servant de type au genre.}

Le Pénérople aumusse. Peneroplis lanatus.

Nautilus planatus. Der flache schiffer. Testac. microsc. a Leo. von Fichtel, etc., pag. 93. tab. 16. fig. d, f. deuxième variété.

Indépendamment des caractères que nous venons de tracer, la coquille qui nous sert de type a de plus une inflexion ou courbureà sa base; ses cloisons très-apparentes forment autant de côtes plus fortement colorées, et finement striées. Quant au reste, cette coquille est encore pellucide, et permet de lire au travers de son têt la série et la disposition des nombreuses cellules de chaque concamération; ces cellules deviennent plus grandes à mesure que la coquille prend plus d'accroissement. Il est probable que leur nombre répond à celui des animaux qui les habitent, et qui les construisent simultanément pour former un nouveau rang. Cette volonté simultanée n'a rien qui nous étonne, parce qu'elle est nécessairement commandée à une époque annuelle 
et réguliere de croissance et de goufloment ou de julithore; mais nous croyous pouvoir indiquer ici une observation qui derient commme a toutes les copuilles cellulées quion trouve constamment privées de leurs animaux sur les plages asséchées des mers, et qui ne flottent pas it la surface de leurs eaux, ce qui en fait des espéces, pour ainsi dire, pélagienues, habitant ì la vérité, non pas les mers les plus protondes, mais toujours aufond des eaux, parce quil faudroit que tous les animaux d'une méme famille eussent instantanément la volouté de remonter à leur surface, et de manouvrer de concert en conséquence; ce qui supposeroit une volonté intellectuclle, communc à tous, et résultant de communications mutuelles : opération toute différente de celle si micanique de la croissance. Cette observation peut encorc sétendre à tous les autres mollusques qui virent en famille dans une habitation qui leur est commune, ainsi qü ù une foule de polypes, dont beaucoup d'ailleurs restent attichés aux rochers du lieu de leur naissance.

La spire du pinérople aumusse est roulée dans le genre de celle des lithuites: ce genre est un des plus marquans de ceux qui servent de passage des coquilles contournées en spirales à celles droites. Sa couleur est jaune, il est perlé, et ses cloisons narquent en rose. On le trouve jeté par la mer sur la plage de Livourne en Etruric. Il a une ligne de grandeur en tout sens. 
Coquille droite, sommet spiré. 


\section{LXVI。GENRE。}

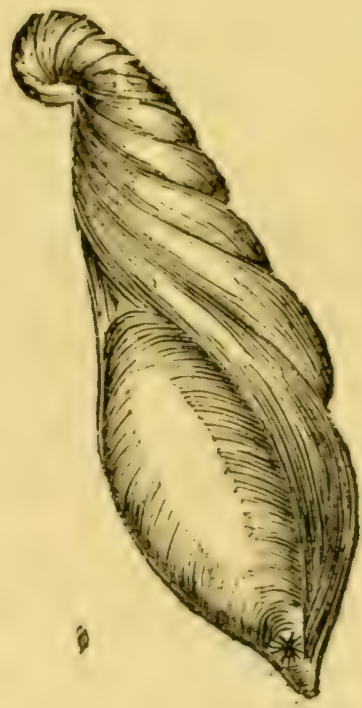

ASTACOLE; en latin, Astacoles. 


\section{L'A STACOLE.}

Caractères génériques. Coquille libre, univalve, cloisonnée; droite, et à sommet spiré; renflée, arquée; dos arrondi; bouche lancéolée, recouverte par un diaphragme bombé, percé à l'angle extérieur par un siphon étoilé; cloisons unies.

\section{Espèce servant de type au genre.}

\section{L'Astacole crepidulé. Astacolus crepidulatus.}

Nautiluscrepidulus. Der pantoffellcehnliche schiffer. Testac. microsc. a Leo. von Fichtel, etc., pag. 107. tab. rg. fig. g, h, i. - Soldani, test. T. I. P. I. pag. 64. tab. 58. fig. b.b. Nautilus lituitatus.

Très-rapproché des lituites, ce genre ne peut cependant point être confondu avec eux, parce que la bouche n'en est point ronde, ni placée à la base sur un plan parallèle à l'horizon; cette bouche au contraire est sur le côté dans une direction perpendiculaire à l'horizon ou verticale, ce qui permet d'assigner un dos aux coquilles qui le composent. Dans l'astacole crépidulé le dos n'est ni armé ni caréné; mollement arrondi, il indique par des renflemens successifs, le nombre des chambres ou 
concamerations dont la coquille est composcée. Les astacoles sout une des nuances intermédiaires entre les coquilles cloisonnées, contournées en spire, et celles qui ne le sont qu'à leur sommet. Cette nuance est d'autant plus marquuée que l'astacole est légérement arqué, et ‘fu'il est très-rapproché des lituites. Dëjà cette observation avoit été saisie par le célèbre Linnée, qui indiqua ces coquilles alongées, mais un peu arquées, sous la dénomination gínérale de nautiles elongati, en y rapportant toutes celles qui partageorent celte conformation.

L'astacole que nous décrivons est diaphane et vitré, un peu aplati sur les flancs, et son sommet fortement recourbé, est replié et adhérent au corps de la coquille.

Sa couleur est d'um blanc de perle, teintée en oranger, surtout aux indications des cloisons, qui sont fortement prononcées.

On trouve l'astacole crépidule sur la plage de Lirourne, en 'Toscane, et dans toute l'Adriatique.

Il a une ligne de longueur. 


\section{Coquille droite, à sommet spiré.}




\section{LXVII'. GENRE。}

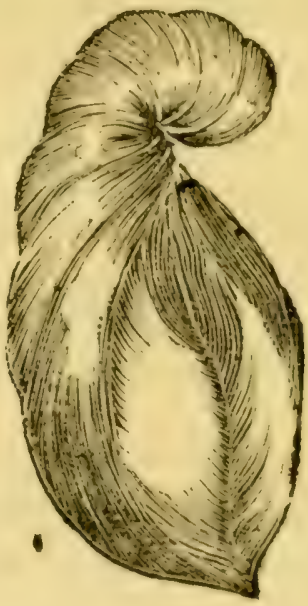

CANCRIDE; en latin, Caxcris. 


\section{LE CANCRIDE.}

Caractères génériques. Coquille adhérente, univalve, cloisonnée; recourbée au sommet, mais droite en s'avançant vers la base; bouche lancéolée, recouverte par un diaphragme bombé, et divisé dans sa longueur par une raie ou fente; têt arrondi, dos caréné vers la base; cloisons unies; siphon inconnu.

\section{Espèce servant de type au genre.}

\section{Le Cancride oral. Cancris auriculatus.}

Nautilus auriculus. Der ohrfermige schiffer. Testac. microsc. a Leo. von Fichtel, etc., pag. I Io. tab. 20. fig. $d, e, f$.

Ce genre s'écarte du précédent, malgrẻ toute l'analogie qu'il pourroit avoir avec lui, parce qu'au lieu d'un siphon angulaire et étoilé, sa bouche présente au con. traire une longue fente ou raie qui, probablement, remplace ce siphon; sa base se termine en pointe, et le dos rond jusquà elle se carène en formant une espèce de bec avancé; du reste la coquille servant de type est assez ventrue et comme soufflée.

Extrêmement transparente par sa pellucidité, elle laisse lire au travers de son têt la disposition de ses cloisons; et on la trouve sur les algues, les fucus et les crustacés de la Méditerranée, sur lesquels cependant elle est assez rare, quoiqu'elle paroisse préférer les langoustes pour se placer sur leur cruste ou têt. 
Cette observation nous conduit à regarder le cancride oral comme annucl, c'est-i-dire croissant, vivant et terminant sa carrière dans le cours d'une année, terme quilui seroit commun avec beaucoup d'insectes, de plantes, et plusicurs autres ctres; et alors on ne peut qu'ètre étonué de son prompt accroissement, car nous lui arons reconnu jusqu'i vingt-neuf cloisons, formant autant de concamérations. Si nous n'eussions rencontré le cancride oral que sur des algues et des fucus, nous n'aurions point pu délerminer aiusi son igge à quelyues égards; mais dis rue nous l'eumes reconnu sur le têt des crustacés, et principalement des langoustes, qui, comme l'on sait, changent ce têt ou cruste chaque année a l'époque du solstice d'été, nous avons dù en inférer naturellement que ce mollusque testacé cloisonné périssoit à cette époque, qui pour lui devient nicessairement le terme de son existence : et quelque soit l'étre auquel il s'attache en parasite, que cet étre soit crustacé ou végétal, comme il n'y atteint toujours qüà la dimension d'un tiers de ligne, et que jamais on ne la vu plus grand, il est tries-probable quil ne devient point plus âgé sur les algues que sur les crustacés.

Nous croyons devoir encore indiquer pour la synonymie de ce genre, Soldani, test. T. I. P. I. tab. 5o. fig. C. lite. 00. Pp. qq. rr. - et tab. ror. dd. 
Coquille droite, à sommet spiré. 


\section{LXVIII". GENRE.}

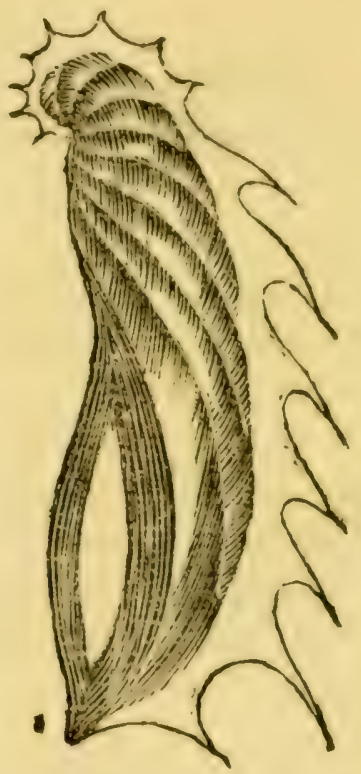

PÉriple; en latin, Prniples. 


\section{LE PERIPLE.}

Caractères génériques. Coquille libre, univalve, cloisonnée, recourbée au sommet, droite en s'avançant vers sa base; bouche lancéolée, recouverte par un diaphragme bombé; t têt arrondi; dos caréné et armé; cloisons unies; siphon inconnu.

\section{Espèce servant de type au genre.}

\section{Le Périple élongé. Periples elongatus.}

Polythalames de Soldani. Testac.tab. 58. vas. I9o.bb.

Toujours lié, tant avec les genres qui le précèdent qu'avec les autres qui le suivent, celui que nous décrivons n'a point de siphon, ni de raie ou de fente au moins visibles; d'ailleurs son dos est caréné et armé de pointes en manière d'éperon et de crémaillère, caractères qui nous ont paru assez tranchans pour nous décider à l'isoler de tous les autres. La coquille du périple élongé est presque droite, mais le sommet en est spiré, et cette spire se roule contre le têt, auquel elle est adhérente. Les concamérations en sont étroites, parce que les cloisons très-apparentes sont aussi très-rapprochées les unes des autres; quant à son ensemble, la coquille est assez aplatie et carénée ; elle est perlée et irisée, la bouche est aurore, et les cloisons conservent cette teinte dans l'intérieur. 
On trouve le péripte célongé a létat marin, sur les plages de l'Adriatique; et on le rencontere de méme sur la côte occidentale de lìle de Bornćo, dans locran indien; il git encore fossile dans les bancs de la Coroncine, prés de Sienne en Étrurie, et alors sa coulcur est ocracćeet ferrugineuse.

Il n'acquiert ordinairement qu'me demi-ligne de longueur. 
Coquille droite, à sommet contourné. 


\section{LXIX: GENRE.}

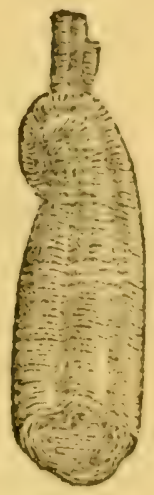

NOGROBE; cn latin, Nognouss. 


\section{LE NOGROBE.}

Caractères génériques. Coquille libre, univalve, cloisonnée, droite, et à sommet contourné; bouche trefflée ou festonnée, horizontale; cloisons coniques, festonnées et percées par un siphon central.

\section{Espèce servant de type au genre.}

Le Nogrobe vermiculé. Nogrobs vermicularis.

Tuyau vermiculaire testacé à quatre pans, etc. Ḱnorr, recueil des monumens des catastrophes, etc. vol. I I. sect. I I. pag. 255. pl. I. a. fig. 8.

L'auteur que nous venons de citer dans notre synonymie, en nous servant de l'édition françoise, a donné une assez mauvaise figure du nogrobe qui nous sert de type. Elle a à-peu-près huit lignes de longueur, et en général elle est peu caractérisée. Cette pétrification curieuse lui avoit été communiquée par M. d'Annone, de Bâle en Suisse, comme pouvant être rangée parmi les vermiculites, et Walch, rédacteur du texte de l'ouvrage de Knorr, qui n'en vit que le dessin, a suivi aveuglément cette indication. Cependant il auroit dû y reconnoitre une coquille cloisonnée, puisque ces cloisons sont appa- 
rentes à l'extéricur, ce qui lui a lasit dire "qu'on voit à l'extrémité la plus large deux replis, comme si le têt ayoit deux fois été retroussé en arrière ". Aussi heureux yue le docteur d'Annone, nous possédons une pétrification semblable, mais plus grande, et qui vient d' $\mathrm{Im}$-. boine, tandis que celle décrite par WValch venoit de Muttenz, village du canton de Bâle.

Notre nogrobe vermiculé est spathique, conme le sont toutes les bélemnites, arec qui il a beaucoup d'analogie: il ressemble extérieurement ì de la corne, et donnc une tris-mauvaise odeur quand on le frotte. Ses cloisons sont encapuchonnées les unes dans les autres en manière de cliausses à filtrer. Il a une teinte plombée, bleuâtre, mais ferrugineuse.

Le nogrobe vermiculé d'Amboine a un pouce et demi de long; on ne le comnoit encore qu’à l'état fossile. 
Coquille droite, is sommet spiré. 


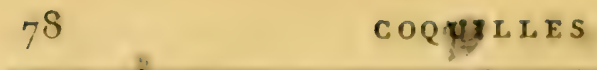

\section{LXX $X^{e}$ GENRE.}

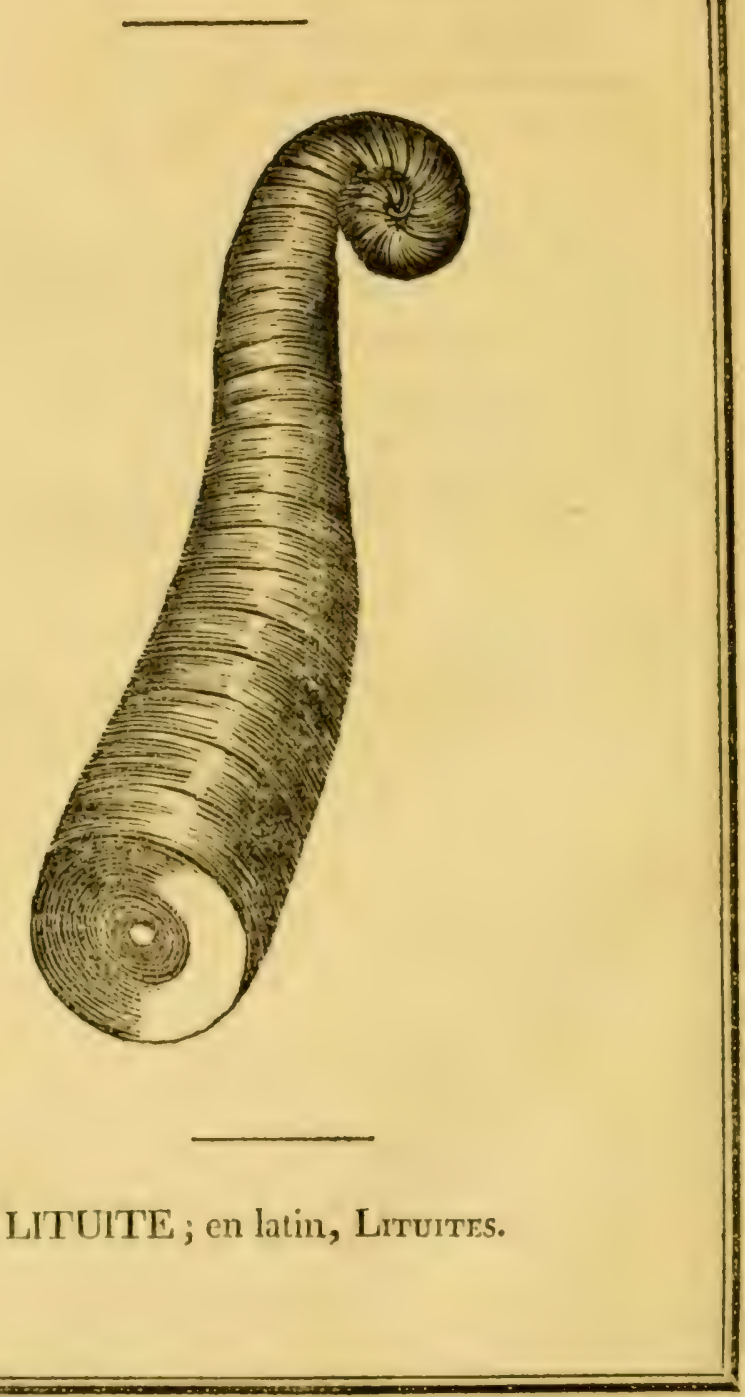




\section{LE LITUITE.}

Caractères génériques. Coquille libre, univalve, cloisonnée; recourbée au sommet, mais droite en se prolongeant vers la base; bouche ronde, ouverte, horizontale; cloisons unies, percées par un siphon central; la spire du sommet adhérento au têt.

\section{Espèce servant de type au genre.}

\section{Le Lituite augural. Lituites lituus.}

Breyn de Polythalamiis, pag. 27. Jacq. Théod. Klein, de tubulis marinis, pag. 1 o. - Lituites, tubulis concameratis, apice spiræ modo intorto. - J. Fr. Leopold, relatio. de itinere suevico, London, I 720. Hill. natur. hist. of fossils. p. 620. - J. W. Baumer, natur des mineralreichs. p. 321. fig. 21. - Ceratoïdes articulatus Scheuchzeri. - Radii articulati lapidei c cono curvati, vel ad apicem spiræformes. Waller. Lituites. Bertrand dict. oryctol, - En allemand, Lituiten, bischofsstæbe, gebogene orthokeratiten. - En hollandois, Lituit, bisschop staef. - En belge, abt'sstock.

Les lituites sont aujourd'hui trop connus des concliyliologues pour que nous croyions devoir entrer ici dans de plus grands détails, quant à leurs formes, que nous croyons d'ailleurs avoir suffisamment déterminées par notre figure, notre phrase caractéristique, et la courte synonymie que notus avons donnée à l'appui, et dans laquelle nous n'avons pas cité la vingtième partie des 
auteurs qui en ont traité, atcendu que tous les oryctologrues en général en ont parlé. Jusqu'a ce jour nous ne connoissons les lituites qu'à l'état de peitrification, el presque toujours repris dans des marbres coquillicrs, avec des ammonites, des belemnites, et une foule d'autres êtres pélagiens. Il est pour nous sans exemple quion en ait rencontrí il l'état marin, et nous nous sommes vu forcé en conséquence d'abandomer ici l'un de nos gruides ordinaires (I) qui a rattaché les lituites au genre spirule, dont cependant ils s'écartent autant que peut le fire une coquille droite d'une coquille contourne totalement en spirale : et quoique l'exemple donné par M. De la Marck ait été suivi par M. Felix de Roissy (2), nous ne pourons point partager leur opinion, aric dautant plus de raison que la spire du sommet des lituites est adlıérente au têt, et qu'en suivant le systime de ces auteurs, on devroit plutòt alors jeter les lituites parmi les cornes d'Ammon. Nous connoissons des lituites de deux pieds de longueur. On les rencontre surtout dans les marbres rouges rlOEland en Thuringe; ainsi qu'on Angleterre et en Écosse. Quelques espueces décrites par Soldani à l'état mariu, semblent venir se rattacher à notre genre.

(1) De la Marck, anim. sans vert. pag. soz. Genre spirule.

(2) Felix de Roissy, cont du Buffon, édit. de Sonnini. hist. des moll. tom. 5. pag. 14. geure spirule. 
Coquille droite, is sommet spiré. 


\section{LXXI․ GENRE.}

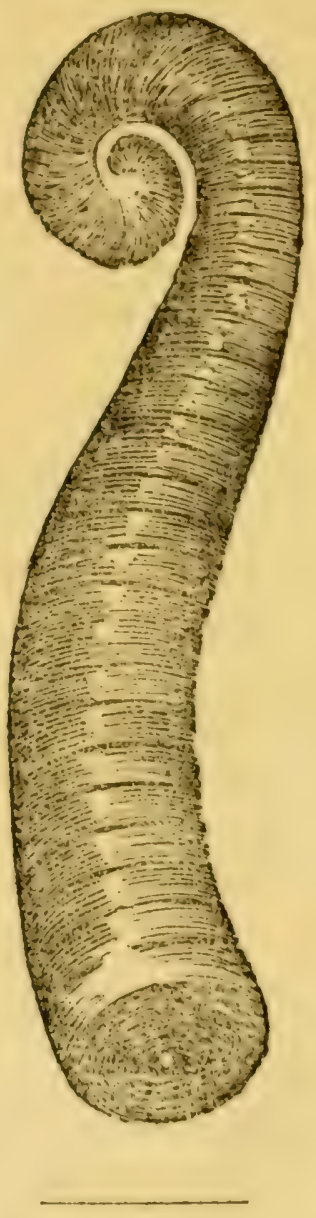

IOR'TOLE; ; latin, Horrorces. 


\section{L'HORTOLE.}

Caractères génériques. Coquille libre, univalve, cloisonnée; recourbée au sommet, mais droite en se prolongeant vers la base; bouche ronde, ouverte, horizontale; cloisons unies, percées par un siphon central ; la spire du sommet évidée et non-adhérente au têt.

\section{Espèce servant de type au genre.}

\section{L'Hortole crossé. Hortolus convolvans.}

Quoique cette coquille fossile ait été constamment confondue par tous les auteurs avec les lituites, nous avons cru, fondés sur sa spire évidée et non-adhérente, pouvoir en faire un genre particulier; et en effet, n'y sommes-nous pas autorisés par la division déjà faite par tous les conchyliologues, des cornes d'Ammon ou ammonies et des spirules, dont la différence caractéristique est d'avoir tous les tours de spire isolés et non-adhérens? C'est donc cette considération qui nous a porté à fendre et séparer le genre lituite en deux sections, à l'une desquelles nous avons conservé l'ancienne dénomination, pour donner celle d'hortole à la seconde. Comme les lituites, les hortoles sont très-nombreux, mais seulement à l'état fossile, car on n'en connoît pas à l'état marin ; comme eux ils rappellent par leur forme recourbéc le lituus, ou bâton augural des anciens, et qu'on retrouve encore de nos jours dans les mains des évèques et des abbés. 
Les hortoles acquierent tout autant de dimension que les lituites: on en connoit qui viennent de la Cline; celui qui nous sert de type fait partie d'un fragment de marbre noir, espéce de pierre puante des environs de Namur. Lorsque les concamérations, alvéoles ou cloisons de l'hortole se détachent, elles ressemblent, comme celles des lituites et de quelques autres pétrifications, à des verres de montres; et dans cet isolement, elles en portent le nom chez quelques lithologues.

La couleur de lhortole crossé est condrée; et ses cloisons, comme son têt, se dessinent en blanc, parce qu'ils sont convertis en spath calcaire; cependant, comme toutes les autres, ces pétrifications partagent plus ou moins la teinte des lits ou couches dans lesquelles elles gissent ; ocracées, dans les terrains ocracés; pyritiscées, dans ceux sulphureux et vitrioliques. 
Coquille cloisonnée, droite. 


\section{GENRE.}

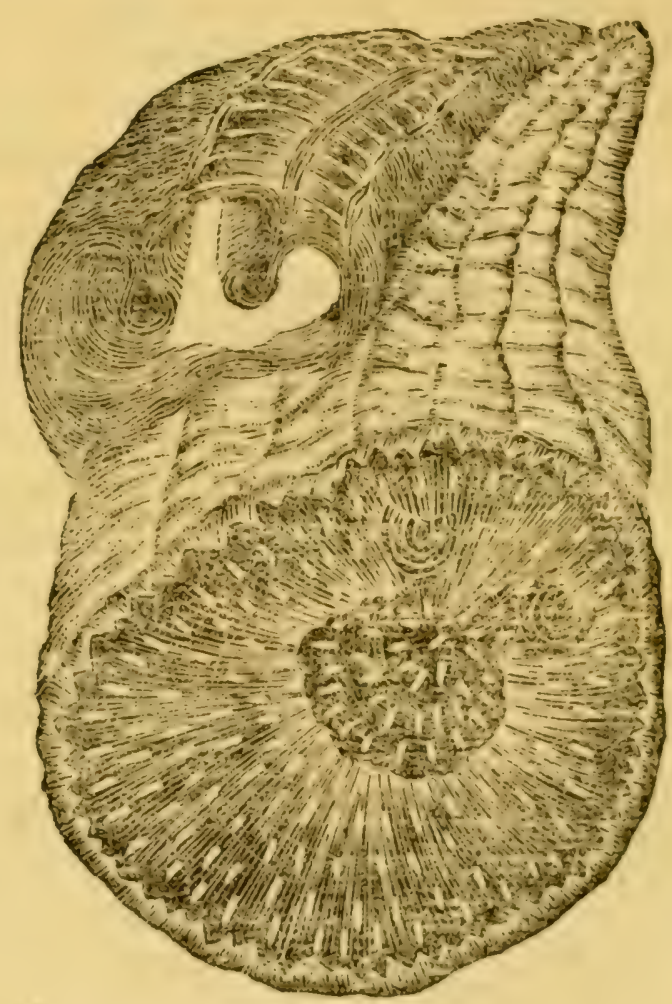

IIIPPURITE; en latin, In'peritrs. 


\section{L'HIPPURITE.}

Caractères génériques. Coquille libre, ou adherente, univalve, cloisonnée; droite ; conique et arquée ; bouche arrondie, peu profonde, ouverte, horizontale; cloisons criblées et percées latéralement de deux grands stigmates, répondant à deux arrêtes parallèles ou divergentes, qui percent toutes les cloisons jusqu'au sommet de la coquille.

Espèce servant de type au genre.

\section{L'Hippurite bioculé. Hippurites bioculatus.}

Orthoceratithes. - Picot de la Peyrouse, Monographie des orthoc. Schrœter. lithol. lex. - Orthoceratitæ. Orthoceratiti. Orthocerothes. Tubulus concameratus. Radius articulatus. Cauda cancri. Helmintholithus nautili orthoceræ. - En allemand, Orthokeratiten; vielkammerige tubuliten; gerade hœrner.

De la Marck, anim. sans vert. pag. 1 o4. genre hippurite. - Felix de Roissy. Suite à l'hist. nat. de Buffon, édit. de Sonnini, hist. des moll. tom. 5. pag. 40. genre hippurite. - William Thomson. Cornucopice. Biblioth. brit. no. I 5o. pag. 247. fig. - Journal de phys. an. 1802.

Assez généralement les oryctologues ont confondu les hippurites avec une foule d'autres pétrifications, dont beaucoup tiennent aux madrépores. Picot de la Peyrouse est le premier qui nous ait donné de bonnes figures de ceux qu'il recueillit dans les Alpes, et qu'il publia sous 
le nom d'orthoceratites; De la Marck, suivi prar Felix de Roissy, et maintenant par nous, lemr doma le nom d'hippurites, afin de mieux les caractiriser, et de faire plusieurs coupes devenues nécessaires dans le travail de la Peyrouse. ( uant au docteur Thompson, qui voulut décrire lihippurite quion trouve en Sicile, dans les couches calcaires du cap Passero, cap Pachymus des anciens, et ì qui il domna le nom de cornu copice, on regrette que cet auteur n'ait pas été plus limiliarisé arec le sujet qu'il traitoit.

M. Desmarets a rapporté des Pyrénces beaucoup d'hippurites; ils doivent encore exister dans quclque dépût du Jardin des Plantes de Paris, oi ils restent en-. fouis, ce savant, d'ailleurs si estimable, s'ítant refusé à les publier, ou à les laisser publier par d'autres jusqü à présent. Puisse cette lacune être remplie!

L'hippurite bioculéa un têt tres-épais : il vit souvent en famille, parce qu'on le trouve fréquemment groupe; il n'est comnu qu'a l'étatfossile, ordinairenent spathique et decouleur ocraccéc. Nous en arons vu d'un pied et demi de diametre; et quoique MM. De la Marck et de Roissy lui aient donné un opercule, nous regardons cet opercule comme cloison; et aprés le plus mùr examen nous persistons dans notre opinion. 
Coquille droite, spire arquée. 
LXXIII. GENRE.

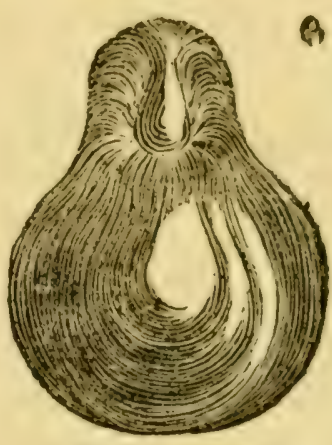

CANOPE; en latin Caropts. 


\section{LE CANOPE.}

Caractères génériques. Coquille libre, univalve, cloisonnée ; droite, à sommet arqué et base globulaire ; bouche inconnue; cloisons unies; siphon inconnu.

\section{Espèce servant de type au genre.}

\section{Le Canope fève. Canopus fabeolatus.}

Cette coquille qui ressemble à une fève, ou même plutòt à une de ces perles en poire, que nos pères nommoient perlerines ou pélerines, d'après celles qui ornoient les oreilles de Cléopâtre, et dont, une fut dissoute dans du vinaigre par cette belle reine d'Égypte, est parfaitement diaphane; sa transparence permet de voir au travers du têt la disposition des cloisons; elles sont placées régulièrement les unes au-dessus des autres, comme le sont celles des nautiles et des ammonies ; c'est à-dire un peu arquées, ce qui pourroit faire regarder le sommet, qui de même n'est point roulé, mais arqué, comme remplaçant dans les canopes le retour de spire contourné que nous ont présenté les coquilles dont la volute étoit contournée en spirale. Le canope fève extrêmement renflé à sa base, y acquiert presque tout d'un coup des dimensions doubles de celles qui caractérisent le sommet; l'un et l'autre sont parfaitement arrondis et presque globulaires.

Ce canope offre des coulcurs charmantes : il est glacé et teinté de toutes celles du prisme. Jeté sur la côte par 
les flots, il perd bientôt toutes ces muances ; il blanchil, devient firineux, et alors c'est une copnille morte. Nous sommes en génćal bien loin de comnoitre encore les couleurs intégrantes, vivantes et primitives des corguilles; et surtout de celles qui sont naturellement polies par les mollusques qui les forment, et qui le sont antant au dohors qu'au dedans. On preut cependant s'en faire une idées prar celie que l'art díprouille, et dont il fait refléter toute la nacre et ees teintes si riches qui forment l'ornement de nos calbincts ; et néanmoins ces belles teintes sifraiches, si pures, dues à l'art, sont bien loin encore du brilliun de celles prodiguées par la nature à une foule de contulles cloisonnées et microscopiques. Ce n'est que daus l'eau, dans l'état de vie, quil est possible de s'en firire me idće; et alors mème leurs teintes si brillantes, sont si fingilives, qu'elles perdent une grande partic de leur orient it la mort des animaux qui leur donnerent l'être.

Le canope fève se trouve sur les côtes de l'ile de Java, et sur celles de quelques autres îles de l'Océan indien.

Il acquiert quelquefois une ligne et demie de longrueur.

Nous croyons que queleques coquilles figuries at décrites par Soldani, viemment se ranger sous ce genre. 
Coquille droite, dos armé, 
LXXIVe. GENRE.

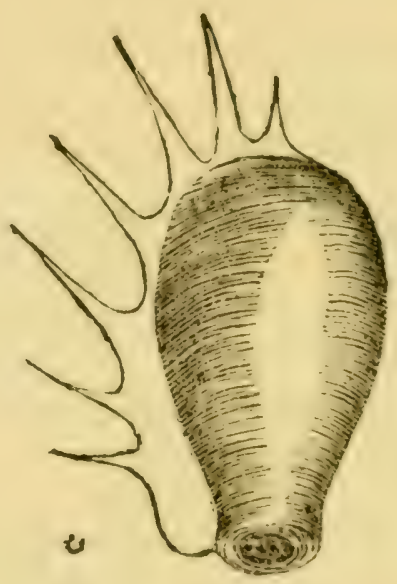

MISILE; en latin Misilus. 


\section{LE MISILE。}

Caractères génériques. Coquille libre, univalve, cloisonnée; droite et formée en cruche un peu aplatie; carénée et armée sur un des còtés; bouche ovale, ouverte; cloisons unies; siphon inconnu.

\section{Espèce servant de type au genre.}

\section{Le Misile aquaire. Misilus aquatifer.}

Polythalames de Soldani, tab. III. vas. 24r. Y?

Les misiles ressemblent par leurs formes à un vase à boireayant un col étroit ; on ne peut mieux les comparer qu'à ces gourgoulettes dont on se sert dans les pays chauds pour rafiraichir l'eau destinée à la boisson. Ils ont de plus une crête ou carène fortement armée d'épines ou de pointes. Dans le misile aquaire ces pointes sont longues, effilées et semblables à celles de quelques rosiers, qui ont les épines très-allongées; toute la coquille est transparente, mais la carène ou armature est beaucoup plus diaphane et plus vitrée encore que tout le reste. Cette carène ne garnit qu'un seul côté en remontant jusqu'au sommet qui est arrondi; et nous croyons pouvoir regarder cette armure comme indiquant encore le dos, 
indication que bientit nous verrons disparoitre tolalement, surtout dans les coquilles droites et cylin driques.

Les coulems de la coquille qui fait le type de ce genre, sont les mimes que celles qui constituent l'irisation; elle partage à cet égard, de la maniére la plus brillante, les teintes de quelques-unes de celles qui précèdent.

On trouve le misile aquaire à l'état marin et à l'état fossile: fossile il est somvent ocracé, et sà couleur se ressent des bancs dans lesquels il est ensevcli.

Il vient de l'Adriatique; et il acquiert une ligne de diametre. 
Coquille droite; dos bombé. 


\section{LXXV: GENRE.}

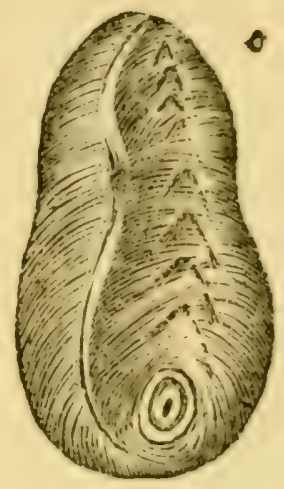

CANTHARE; en latin, Cavtuiarus. 


\section{LE CANTHARE.}

Caractères génériques. Coquille libre, univalve, cloisonnée; droite; formée en nacelle, arrondie sur le dos, aplatie sur le ventre; obtuse au sommet, plus large ì la base; bouche ronde, placée latéralement vers la base ; cloisons en triangle ou chevronnces ; siphon central.

Espèce servant de type au genre.

Le Canthare sabot. Cantharus calceolatus.

Soldani, testac. tab. I 07. vas. 239 . pp.

D'après les caraclères que nous venons de tracer, on voit que les canthares sont dessinés en sabot, en pirogue, en mandoline ou guitare, formes d'autant plus singulières quils ne présentent aucun retour de spire, le sommet étant parfaitement obtus. Par suite de cette conformation le veutre est absolument plat; il présente l'indication des cloisons toutes disposées en chevrons vers leur partie supérieure, et formant l'onglet en se rendant vers le dos de la coquille qui est parfaitement arrondi. 
Cest ici fue nous parlons pour la derniere fois du dos diune corpuille dans lordre de celles cloisonnies; il lavenir cette partic ne se distinguera plus, a moins quion ne veuille en retrouver les derniers linramens dans le paclite; genre rapproché des belemnites, mais ouvert, limulé et courbé vers le bout. Ce sont ces dígradations qui nous conduisent insensiblement vers la lin de cet ordre, qui s'est étendu sous notre plume, et que cependant nous avous peut-être encore plus resserré que ne l'exigeoit le mode de nos travaux; car nous prévoyons qu'après nous d'autres conchy liologues ajouteront encore à la série des genres que nous avons été forcés de créer, dans une partie que nous regardons comme la plus difficile de la science que nous traitons, et dans larfuelle nous croyons avoir pris linitiative.

Le canthare sabot est perlé et irisé: il vient de l'Adrialique; et il a une ligne de longueur. 
Coquille droite. 


\section{LXXVI . GENRE.}

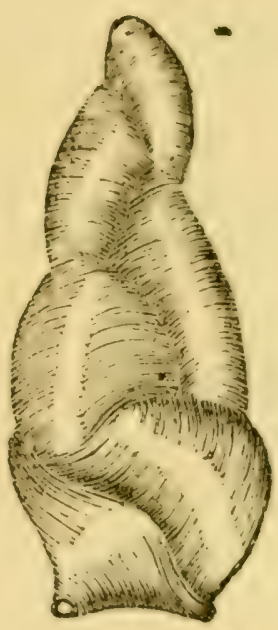

ARETHUSE; enlatin, Aretuzsa. 


\section{L'ARETHUSE.}

Caractères génériques. Coquille libre, univalve, cloisonnée ; formée en grappe; sommet rond ; base élargie; concamérations triangulaires; bouche ronde, placée latéralement à la base; cloisons ondulées; siphon inconnu.

\section{Espèce servant de type au genre.}

\section{L'Aréthuse corymbé Arethus a corymbosa.}

Soldani, testac. tab. 107. vas. 25g. LL.

Nous devons regarder le genre actuel comme l'un des plus curieux et des plus singuliers dont nous ayons traité jusqu'à présent. Les aréthuses sont formés en grappes ou en ovaires, et dans leur ensemble ils présentent, au lieu de concamérations bien régulières, des chambres vésiculées, empilées et adhérentes cependant les unes aux autres. La dernière camération, celle de la base, et qui est la plus grande, s'accole à la pénultième, elle en recouvre la bouche, qu'elle porte à son tour, et il est probable que cette petite bouche ou ouverture qu'on y voit sert en même temps de siphon; l'appendice filiculaire de l'animal occupant toutes les chambres jus- 
yliatusommet. En grincial ces chambres sroupées ont la forme d'un triangle plus ou moins irrégulier, et on pournit les regarder comme étant tordues sur un axe commun, inclìjué à la vérité par cette disposition, mais yui n'existe point matériellenent.

L'arćthuse corymbia un aspect vitreux : il est translucide, irisé, teinté de rouge, d'orangé, de violet, et chaque chambre ou vésicule a pour ainsi dire sa teinte changeante el particuliere. Les cloisons sont assez fortement prononcées.

On rencontre cette corpuille microscopique sur les plages de la mer Adriatique; il est rare de l'y trouver entiere, en raison de sa grande fragilité.

Elle a une demi-ligne de longueur. 
Coquille droite, globulaire, enfilée. 


\section{GENRE.}

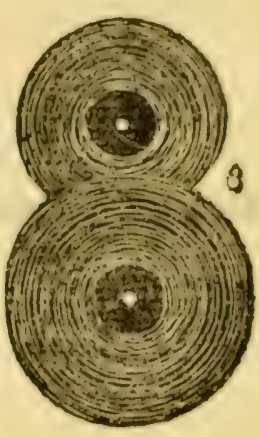

CÉLIBE; en latin, Cuezins. 


\section{LE CÉLIBE.}

Caractères génériques. Coquille libre, univalve, cloisonnée, droite; globulaire et sériale; bouche ronde, latérale; siphon incounu; cloisons bombées et unies.

\section{Espèce servant de type au genre.}

\section{Le Célibe graduel. Chelibs gradatus.}

Nous avons donné à l'une des distinctions qui caractérisent cette coquille, l'épithète de sériale; par cette expression nous avons voulu indiquer la disposition en ligne droite des globes qui la composent; ces globes paroissent enfilćs les uns aux autres, et dans le fait ils sont intimement soudés. Dans la figure qui nous sert de type nous n'avons représenté que deux de ces globules, et nous croyons cependant qu'il en existe souvent bien davantage à la suite les uns des autres; mais comme ce n'est point leur nombre qui nous dirige, mais bien leur disposition, nous rangerons sous ce genre toutes les espèces qui nous offriront deux ou plusicurs globules perpendiculaires et soudés les uns aux autres.

Le célibe graduel observé au microscope, présente une bouche à chaque globule; elles sont rondes, latérales et disposées sur une mème ligne; organisation toute différente de celles que nous avons envisagées jusqu'à présent. Le dernier globule, le plus gros, celui de la base, est implanté sur celui qui le précède, et qui déprime son orbe en y imprimant unenfoncement en forme 
de calote. D'aprés la disposition respective de ces globes, nous arons envisagé chacun d'eux comme formant une concamiration, d'autant plus qu'il est possible que la bouche quion reconnoil dans ceux qui précédent le dernier, sembleroit n'ètre plus ouverte, mais au contraire fermée par une pellicule tellement transparente, que d'aprés l'exiguté de la coquille il est impossible de décider si celle bouche est réellement encore ouverte, ou si elle n'en prisente plus que le stigmate ou la cicatrice.

Le célibe graduel est vitré, un peu sourd cependant de transparence. On le trouve sur les plages de l'Adrialique de couleur rose, teintée de vert, mais flus communément encore entièrement décoloré, comme presque toutes les coquilles microscopiques qui ont subi l'action du soleil.

Il a trois quarts de ligne de longueur, lorsqu'il présente deux globules; dans une série plus complète il doit étre plus long, quoique la taille et l’âge des globules doivent $y$ influer pour beaucoup. 
Coquille droite, intersectée. 
LXXVIII: GENRE。

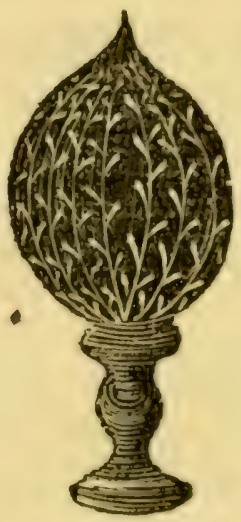

LAGENULE ; en latin, Lagenela. 


\section{LE LAGEN U.LE.}

Caractêres geénériques. Coquille libre, univalve, cloisonnée, droite ; intersectée ; piriforme ; sommet aigu, base aplatie; bouche ronde ; cloisons inégales , unies; siphon inconnu.

\section{Espèce servant de type au genre.}

\section{Lagénule fleuri. Lagenula flosculosa.}

Soldani, testac. tab. I20. vas. 248 . z.

C'est d'après leur ressemblance avec un petit flacon, que nous avons donné aux coquilles qui forment ce genre, le nom de lagénules, en francisant celui latin. Cette conformation est d'autant plus remarquable qu'elle nous indique deux manières d'être différentes pendant la vie des mollusques à qui ces têts appartiennent: dans leur jeune âge, leur demeure est globulaire et folmée en poire, plus tard cette forme change, et des espèces de godets simplantant les uns dans les autres, dessinent un col à plusieurs intersections. Cependant cette particularité n'a rien qui doive absolument nous étonner, car nous retrouverons encore cette manière d'être dans un autre ordre de mollusques; et parmi ceux dont le têt n'est point cloisonné, ne voyons-nous pas les bouches si étranglées des lampes antiques, et de quelques autres limaçons à coquille, dont l'ouverture est dentéc de façon à fermer presque tout passage à l'animal, qui ne 
forme ces dents qur lorsipu il est parvenu à une certaine ¿́poque digraulissement: fait que nous pourrons en. core observer dans les grimaces (murex anus, Linn.), où cutte bouche est plutôt une fissure ondulće et ridée, qu'une ouverture propre à permelte la sortie de lanimal.

Le lagínule fleuri est de plus joliment orné de côtes fenillies; plles indiquent les cloisons du premier âge, qui sont perpendiculaires à l'horizon, et disposées comme celles qui partagent la chair dune orange; celles du col sont horizo:talıs. 'Toute la coquil!e est perlée, teintéc et irisée. On la troure daus les sables de l'Adriatique: elle a pour grandeur un point géométrique. 
Coquille droite, globulaire, implantée. 


\section{LXXIXe. GENRE。}

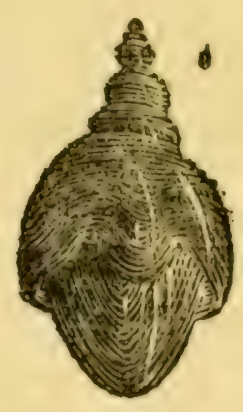

GLANDIOLE; en latin, Glavdoluss. 


\section{LE GLA NDIOLE.}

Caractères goénériques. Coquille libre, univalve, cloisonnée ; droite, implantée et forméc en gland; sommet pointu, central; cloisons glandiformes et multipliées dans chaque gland; siphon inconnu; bouche environnante et festonnée.

Espèce servant de type au genre.

\section{Le Glandiole étagé. Glandiolus gradatus.}

Soldani, testac. tab. I I 7 . vas. 244 . r.

Au commencement de la vie du mollusque qui construit cette coquille, elle est pour ainsi dire imperceptible; peuà peu elle augmente de volume avec l'âge, et les glands dont elle est formée s'étagent en s"implantant les uns sous les autres. Il paroît même qu'à certaines époques, peut-être à chaque année, l’animal qui l'habite augmente sa demeure d'un étage en quittant l'ancien; car chacun de ces étages forme à lui seul une coquille chambrée, chaque gland pris isolément en renfermant plusicurs autres, libres vers la base, mais adhérens vers le sommet. Ces chambres environnantes ou recouvrantes se forment insensiblement et par festons, l'animal construisant les cloisons en les implantant sur le gland précédent, et en les conduisant insensiblement en forme de calice ou de cupule vers la base, jusqu’à ce qu'elles y soient fermées; et c'est l'espace 
laissé entre la cloison extérieme non-terminée, it celle intéricure dejja achevée que nous arons nommi bouche. Il est probable quil faut à ce molluspre la révolution entiere d'une année pour former ainsi un gland entier, ou sont renfermées les cloisuns intéricures, comme l'est l'amanke d'un gland dans sa coque, et qu'aprés avoir aiusi bâti un nomine (juelconnue de ces cloisons, il soil forcé par suite de son organisation d'en commencer une nouvelle sćrie en abandounaut l'ancienne. Rien même ne s'oppose à ce que nous croyions que tous ces glands et toutes leurs cloisons soient percées par un siphon, et traversés par un muscle ou nerf fistulaire qui se rende jusqu'au sommet, les glands et les concamérations grossissant avec l'âge.

Le glandiole étagé est transparent et irisé; on le trouve dans les sables de l'Adriatique, où il a jusqu'à une demiligne de longueur. 
Coquille droite, sommet arqué. 
LXXXe. GENRE.

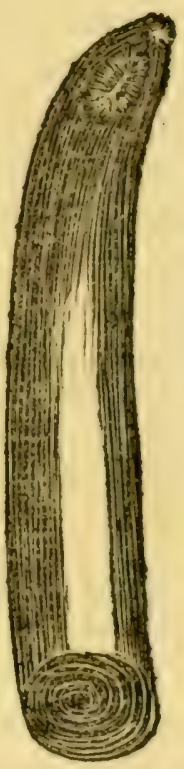

PACLITE; en latin, Pacrites. 


\section{LE PACLITE,}

Caractères génériques. Coquille libre, univalve, cloisonnée; droite et arquée; bouche arrondie, ouverte, horizontale; siphon central ; sommet recourbé , percé par un spincter étoilé , accompagné d'une rimule plissée et placée latéralement ; cloisons unies.

\section{Espèce servant de type au genre.}

\section{Le Paclite biforé. Paclites biforatus.}

Belemnite à pointe recourbée, dont l'extrémité est percée d'un pelit trou circulaire; au-dessous de la partie recourbée se voit une ouverture étroite et oblongue. Knorr. monumens, etc., T. I I. sect. 2. pag. 242. pl. I*. fig. 7 .

Indépendamment de la courbure, le spincter terminal et la rimule qui l'accompagne, nous ont décidé à retirer cettecoquille cloisonnée des belemnites, parmi lesquelles Walch l'avoit rangée. Cetie organisation est d'autant plus remarquable, qu'elle nous permet enfin d'essayer à classer les belemnites, qui offroient jusqu'á nousune des parties les plus difficiles et la plus abandonnée peut-être de tout le règne conchyliologique. Ce genre se dessine purement et avec fermeté, en nous démontrant quavec du temps, de l'étude, de la patience, on vient à bout de surmonter beaucoup de difficultés. 
La coulcur du paclite biforé est bicuatre ce cenclree, ses ouvertures sont teintées de jaune ; il est spa!hique, comme le sont les belemnites. Celui décrit par Walch, provenoit du cabinet de M. Jacques d'Annune de Bâle, qui l'avoit reçu de Prattele!r. Il avoit un pouce sis lignes de long sur un diamètre de six lignes ; ce n'étoit qu'un fragment. Celui que nous présentons à nos lecteurs a deux pouces six lignes sur huit lignes de diamètre; il vient de l'Afrique, du désert du Zaara, et c'est à M. Lesfontaines, administrateur et professeur du Jardin Impérial des Plantes, qui le rapporta avec beaucoup d'au. tres fossiles, que nous en avons lobligation.

On ne connoît le paclite biforé qu'à l'état fossile. 


\section{Coquille droïte, arquée.}




\section{LXXXIc. GENRE.}

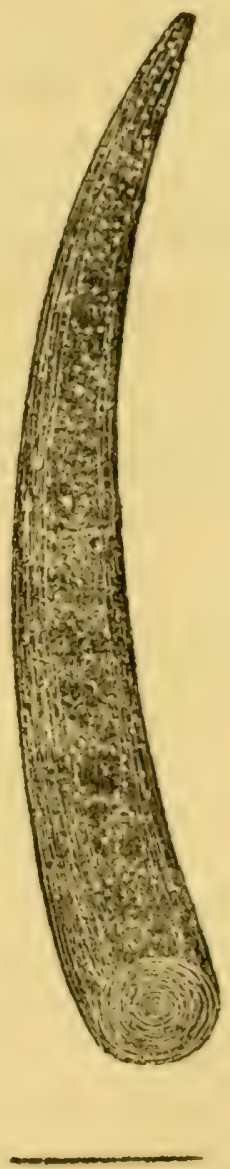

THAL AMULE; ; en latin, Thalamus. 


\section{LE THALAMULE。}

Caractères génériques. Coquille libre, univalve, cloisonnée ; droite et arquée ; bouche arrondie, horizontale, ouverte; siphon central ; cloisons unies; le têt entièrement criblé à l'extérieur par des pores dessinés en cercles autour d'un pore central.

Espèce servant de type au g'enre.

Le Thalamule polimite. Thalamus polimitus.

Knorr. T. III.IV. Suppl, tab. IV. fig. 8 et 9. pag. I 46. - Belemnites polimitus de Scheuchzer.

Dans les coquilles cloisonnées et contournées en spirale nous en avons vu qui se montroient criblées de pores à l'extérieur. Il est vrai qu'elles n'étoient que microscopiques, et qu'en général on pouvoit y craindre une illusion d'optique; mais ici la même modification de la matière calcaire, se montrant sous un grand volume, donne maintenant le cachet à nos premières observations, observations que d'ailleurs nous partageâmes avec des hommes faits pour être cités comme autorités dans cette partic. Les pores qui recouvrent le thalamule polimite se dessinent joliment en cercles autour d'une foule de points ou pores plus gros qui forment autant de centres pour chacun de ces petits systèmes; ce qui fait paroitre toute la surface de la coquille comme recouverte par une très-élégante broderie. 
Knorr, d'accord arec Walch, est un des premiers auteurs rui nous aient fait comnoitre cette coquille, ([u’on n’a encore rencontrée qu’à l'état de pétrification et spathique. Scheuchzer en avoit de même fait mention, et tous trois la regardirent comme un belemnite particulier auquel ils domirent une épithète caractéristique, que nous avons conservé.

Le thalamule polimite qui nous sert de type, a trois pouces de longueur sur six lignes de base; il est arqué et fait en dent d'éléphant; comme les leelemnites, il rend une odeur de foie de soufre lorsiguion le frotte : entièrement spathique, sa couleur est cornée, demi-transparente, et tire sur le grisâtre.

On le trouve en Suisse et principalement dans le canton de Bâle; nais en général il paroît être trèsrare. 
Coquille droite, arquée. 


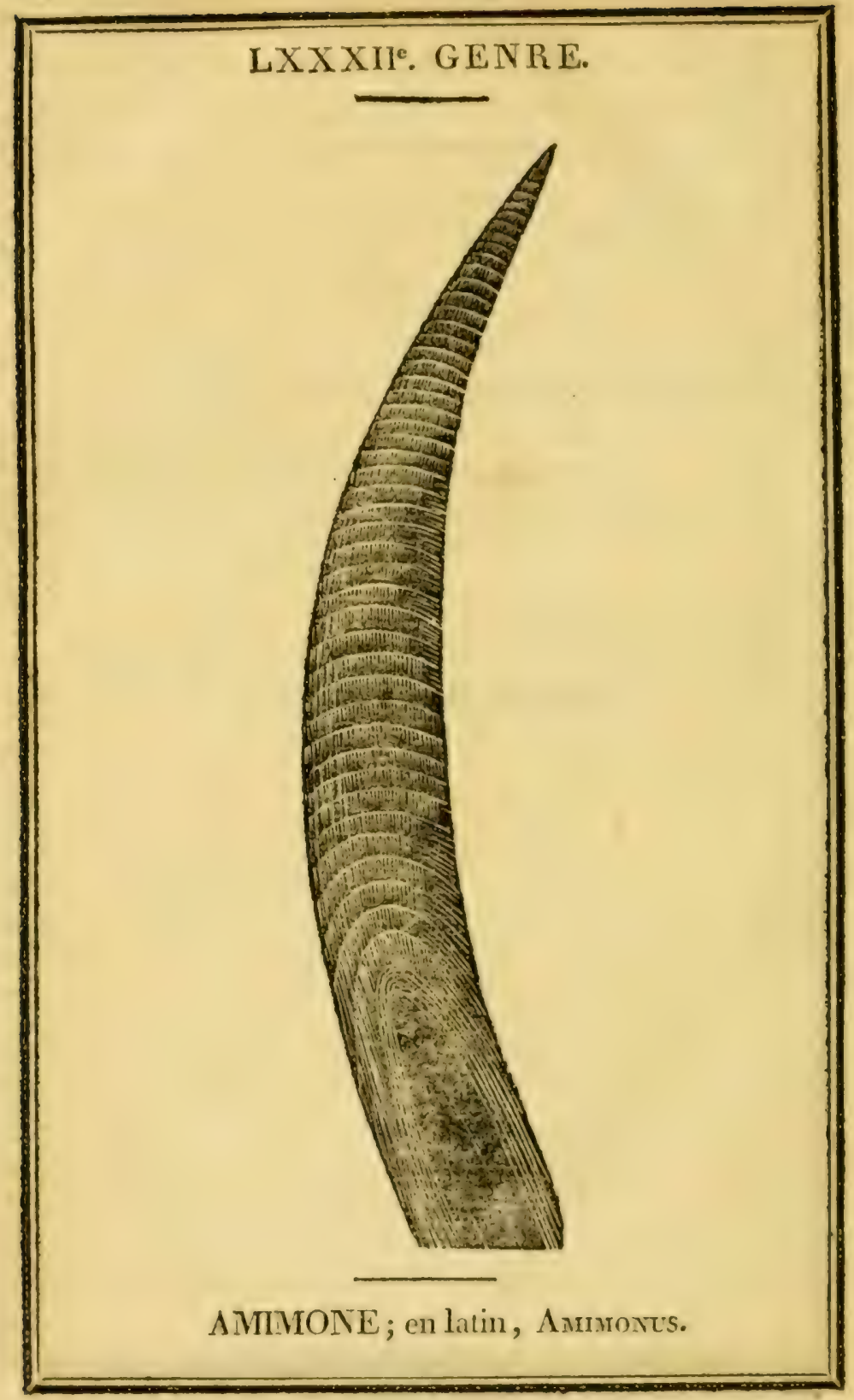




\section{L’AIMIMONE.}

Caractères génériques. Coquille libre, univalve, cloisonnée; droite et arquée ; bouche arrondie , horizontale, ouverte; siphon central; cloisons unies.

\section{Espèce servant de type au genre.}

L'amimone éléphantin. Amimonus elephantinus.

Knorr. Suppl. pl.IV. fig. 2.

En considérant le port arqué de cette coquille, nous nous sommes décidés à en faire la tête d'un genre, cette forme étant nécessairement le résultat d'une organisation particulière dans le mollusque qui la construisit ; c'est pourquoi nous ne pouvons point la ranger avec les belemnites, dont le caractère propre est d'être droit. D'autres auroient pu en faire un belemnite arqué ; mais il est temps enfin de débrouiller la conchyliologie, et surtout celle fossile, en traçant des coupes et en formant des genres partout où il nous a paru nécessaire pour ne point cumuler des êtres disparates qui ne sont point congérières, et dont la plus grande analogie est de modifier la matière calcaire sous une foule de formes, remaniées de toutes les manières et sous tous les modes possibles.

L'amimone éléphantin n'est donc point pour nous un bélemnite, mais il contribue à nous y conduire, et il 
forme un chitimon de la ğrande concaténation générique yne nous nous sommes tracée dés le début de cet ouvrage: il est dialleurs un de ces corps pétrifiés, que nows n'itrons encore rencontrés quà l'état fossile, ot il se trouve, mais rarement, confondu dans les mèmes roches at montagnes calcaires avec les bélemnites, les orthocírathes, ct me quantité d'autres coquilles toutes piligicmes, et dont nous ne comnoissons point encore les analogues. Ou le rencontre principalement à Batstein, et a Altdorff en Suisse, repris et renfermé dans un malbre rouge et un peu argileux; il y est à l'ćtat spathirque, ses concamérations sont remplies d'une pàte brunître, et ses cloisons pressées tranchent très-agréablement, par le blanc de leur cristallisation spathirque, surtout quand on les scie par leur milicu, sur le fond rembruni qui les renferme.

On en trouve de plus de six pouces de longuenr. 
Coquille droite, sinuée, intersectée. 


\section{LXXXII: GENRE.}

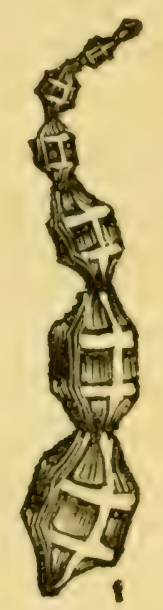

RÉOPHAGE; cnlatin, Reorhax. 


\section{LE RÉOPHAGE.}

Caractères geénériques. Coquille libre, univalve, cloisonnée; droite, sinuée et insectée, ou offrant plusieurs étranglemens, les concamérations augmentant de volume avec l'âge ; bouche terminale, arrondie; siphon central.

Espèce servant de type au genre.

Le Réophage queue de scorpion. Reophax scorpiurus.

Soldani, testac. tab. $162 . \mathbf{K}$.

Ce réophage nous présente une conformation assez particulière pour servir de type à un genre. Rangé parmi les coquilles droites, il est cependant un peu sinueux, mais point tors. Ses chambres sont séparées les unes des autres par autant d'ćtranglemens qui rappellent ceux des insectes, en donnant à leur ensemble l'aspect de la queue d'un scorpion. Elles deviennent successivement plus grandes, singulièrement quadrillées, et on ne peut mieux les comparer qu'à autant de petites lanternes hexagones, enfilées les unes aux autres. Elles sont toutes traversées par un siphon qui lcur est commun, et qui partant de la base ou il sert de bouche, va se rendre au sommet. 
C'est sur la confiance que nous inspire Soldani que nous avons public cette confuille cloisonnce, qui se trouve dans les sables de la mer Adriatidue; ce savant laborieux, doué d'une patience extraordinaire, avoit réuni darss de petits vases des millions de coquilles microscopiques quil décrivoit; il avoit su trier et séparer chaque espéce, et dés-lors au milieu de tant de points de comparaison, on ne peut lui contester les formes quil publia.

Lorsque le róophage queue de scorpion est encore frais dans ses teintes, il présente celles orangeres; exposé au soleil il se décolore, et d'autrefois il prend un ton ocracé.

Il a quelquefois une demi-ligne de longueur. 
Coquille droite, conique, allongée. 


\section{GENRE.}

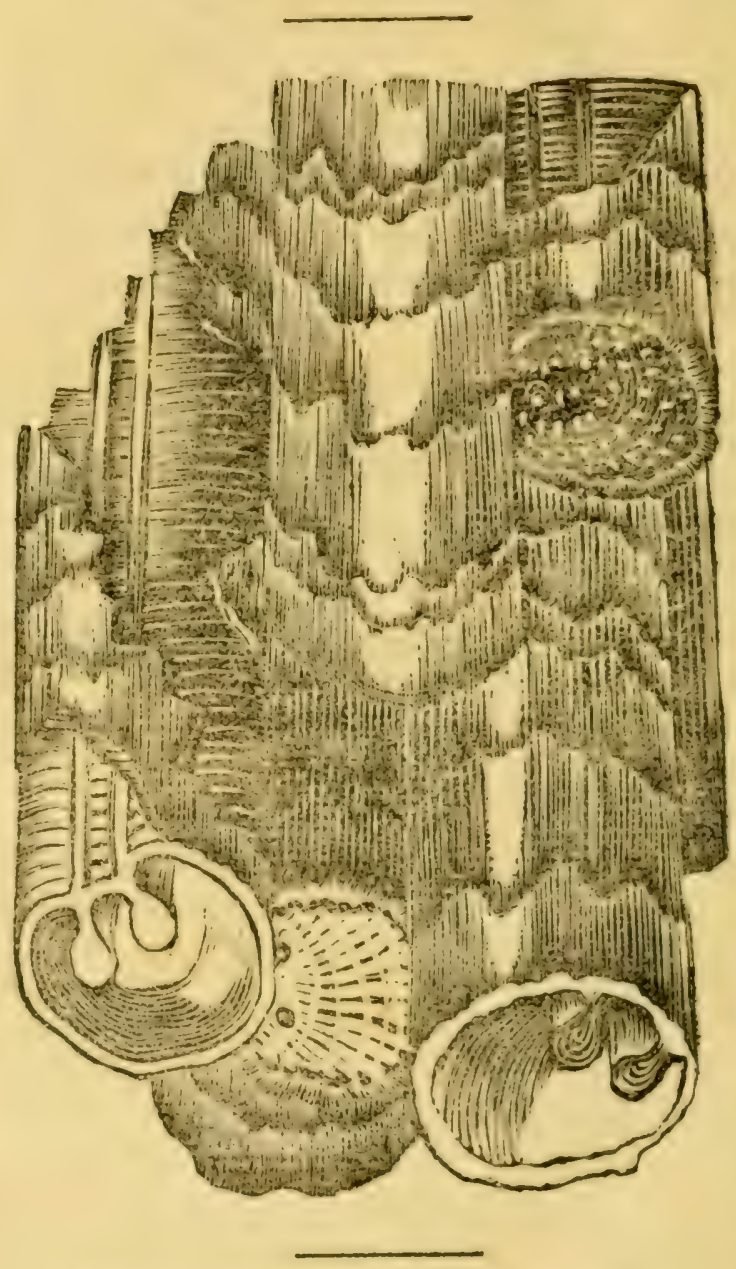

BATOLITE; (in latin, Batolttes. 


\section{LE BATOLITE.}

Caractères génériques. Coquille libre, adhérente, ou vivant en famille, univalve, cloisonnée; droite et fistuleuse; bouche arrondie, peu profonde, ouverte, horizontale ; cloisons criblées et percées latéralement de deux grands stigmates, répondant à deux arrêtes parallèles ou divergentes qui percent toutes les cloisons jusqu'au sommet de la coquille.

Espèce servant de type au gente.

Le Batolite tuyau d'orgue. Batolites organisans.

Voyez Picot de la Peyrouse, monographie des orthoc. Knorr. monumens, etc. T. II. sect. II. pag. 243. pl.

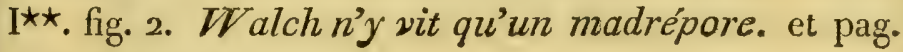
256. pl. I a. fig. I3.

Sous le nom de batolite nous nous sommes décidés à former un genre des coquilles dont nous en présentons une pour type ; c'est un démembrement de celui hippurite, établi par Delamarck, qui coupa de même les orthoceratites de la Peyrouse. L'hippurite est constamment arqué, court et très-épais de têt ; les batolites, au contraire, sont droits, très-longs, et leur têt est plus mince. Au lieu d'être côniques, les batolites peuvent être regardés comme flutés ou fistuleux, parce que dans une dégradation insensible ils acquièrent une très-grande longueur. Nous en avons vu de plus de trois pieds de long, qui n'avoientqu'un pouce de diamètre à leur base, 
ayant à peine perdu deux lignes du côté de leur sommet qui itoi: tronqué; ct comme il existe dans le cabinet de M. de Drée, à Paris, un batulite qui a au moins trois pouces, on peut en inférer que cette coruille isolie ou grouppée avoit, pour acquérir cette dimension, déjà parcouru cinquante-quatre pieds.

On voit dans le batolite tuyau d'orgue, à l'extérieur, les accroissemens annuels et successils de la coquille: ordinairement il est groupé. Dans quelques endroits de notre dessin, nous avons enlevé le têt, afun de laisser entrevoir la disposition des arretes, ainsi que celles des cloisons. Nous persistons à ne point regarder la demiere cloison comme un opercule, ainsi que nous le fumes dans le genre hippurite, cette cloison portant l'empreinte du derriere du corps de l'animal. Pour des yeux inattentifs, rien ne ressemble autant aux madréporites que les hippurites et les batolites.

On ne connoit encore ces deux genres quà l'état pétrifié; ce sont des coquilles jadis pélagiennes, et qui quelquefois à elles seules constituent des masses de rochers dans les Hautes-Alpes. La substance des batolites est spathique, et leur couleur ocracée. 
Coquille droite; campanulée. 


\section{LXXXV'. GENRE.}

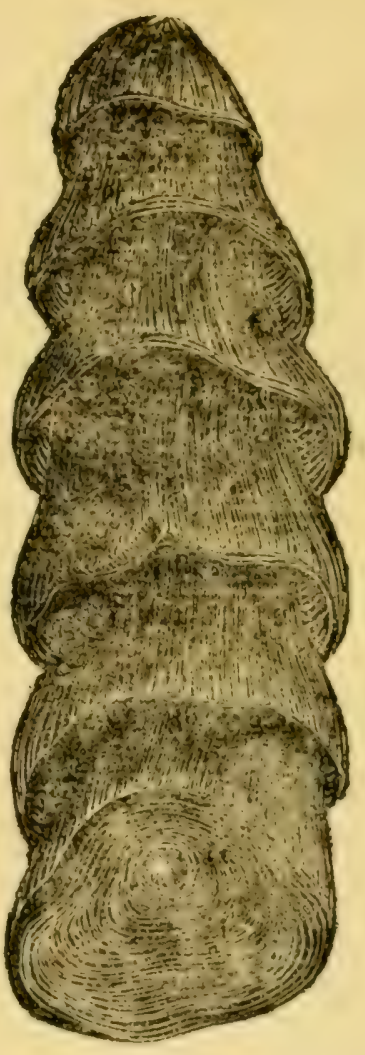

RAPIANISTRE; cn latin, Rapinasister. 


\section{LE RAPHANISTRE.}

Caractères génériques. Coquille libre, univalve, cloisonnée; droite, à sommet obtus; bouche ronde, évasée, horizontale et ouverte ; cloisons évasées, en cloche ou campanulées; siphon central ; point de têt ou de recouvrement général et extérieur.

\section{Espèce servant de type au genre.}

Le Raphanistre campanulé. Raphanister canpanulatum.

Nous avons rencontré cette coquille fossile plusieurs fois en grand nombre parmi les pétrifications que nous avons eues sous les yeux en une foule d'occasions et de circonstances; et nous en avons même pu reconnoître plusieurs espèces. Toutes venoient de la Bourgogne et des environs de Montbard; lieu si riche en corps fossiles que la mine de fer que faisoit exploiter le célébreBuffon, est pour ainsi dire entièrement composée de coquilles pélagiennes à l'état ferrugineux, et notamment de cornes d'Anmon, de bélemnites et de nautilites, parmi lesquels on trouve, quoique assez rarement, le fossile dont il est ici question.

Le raphanistre se distingue des bélemnites et de toutes les autres coquilles droites qui viennent le joindre de droite et de gauche, en ce que son sommet est absolument obtus, que les cloisons sont évasées et campanulées, et qu'il n'a point de têt qui serve d'enveloppe commune; il 
dillere demine des hippurites et des batolites, en ce quan liendes deux aretes intérieures et disposées en goutliure, il ne présente au contraire qu'un seul siphon cencral. Aussi ne le retrouvons-nous point dans l'ouvrage que Picot de la Peyrouse ( 1 ) consacra aux corys fossiles yu'il nomma orthocérathas, mot qui disparoîl peu-it-peu de la conchyliologie, depuis que Lamarck et d'autres autcurs modernes d'après lui, el que nous avons suivis, ont fait le genre hippurite d'une partie des orthocérathes de la Peyrouse, en abandonnant l'autre partie, dont nous renons de faire le genre batolite; car l'orthocire de Lamarck (2) n'est plus une coquille qui fasse partie de celles de la Peyrouse, ainsi que nous le prouverons en son licu.

Le raphanistre de Montbard est calcaire, spathique, teinté de gris et de couleur ocracée; nous en avons vu de plus de six pouces de long.

(1) Description de plusieurs nouvelles espèces d'orthoceralites et d'ortracites, par Picot de la Peyrouse, à Erlang, I $7^{8}$ I, petit in-fol. fig. col. et à Paris, Didot.

(2) Lamarck, Hist. des animaux sans vert. pag. 103. genre orthocère. 
Coquille droite, imbriquée. 


\section{GENRE.}

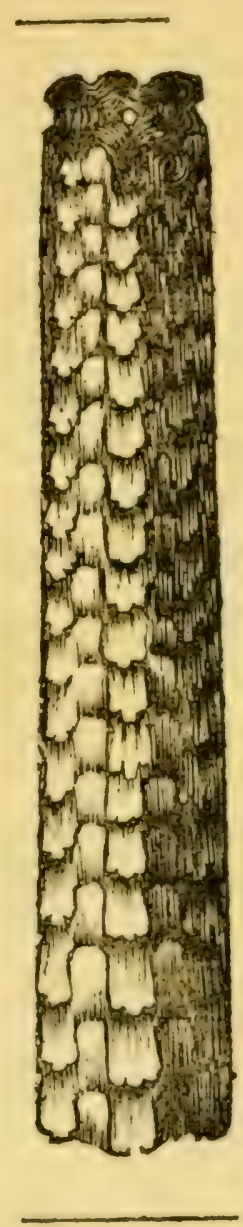

BACULITE; en latin, Bacrlites. 


\section{LE BACULITE.}

Caractères génériques. Coquille libre, univalve, cloisonnée; droite et en cône fistuleux ; cloisons lobées , découpées et imbriquées; bouche arrondie, festonnée, horizontale; sommet pointu; siphon central.

\section{Espèce servant de type au genre.}

\section{Le Baculite vertébré. Baculites vertebralis.}

Corne d'Ammon droite. Faujas de St.-Fond, Hist. nat. de la montagne de St.-Pierre de Maestricht. pl. 2 I fig. 2 et 3. - Bourguet, Pétrif, tab. 49. fig. 3 r 3 à 3 r 6. - Lamarck, Syst. des anim. sans vert. pag. ro3. genre baculite. - Homaloceratite. De Hupsch, desc. de quelques test. pet. pl. IV. - Félix de Roissy, Hist. nat. des moll, faisant suite aux œuvres de Buffon. édit. de Sonnini, tom. 5. pag. 55. genre baculite. pl. Lil. fig. 5. 5.

Ce ne fut qu'après la publication du bel ouvrage sur la montagne de St.-Pierre de Maestricht, par Faujas de St.-Fond, que les conchyliologues se décidèrent à adopter comme genre, et sous le nom de baculite, une coquille fossile que le savant géologue avoit fait graver sous celui de corne d'Ammon droite. Déjà le baron de Hupsch 
en avoit rencontré de soncóté dans les environs d'Aixla-Chapelle, et il les publia de méme sous la dénomination d'homulocératite Le baculite vertébré de Maestricht est calcaire et sablonneus, de couleur jaune, et quoinue toutes les cloisonssoient imbriquées, encheriLrées les unes dans les autres, elles jouent cependant entre elles, parce quil n'en reste plus que le remplissage des concamérations, et que le têt ainsi que les cloisons ont lisparu, en se gazifiant au sein de la terre, entassés avec des montagnes entieres de dípouilles abandonnées par l'antique Occian dans les pays aujourd'hui arrosés par la 1) Ieuse. Nous croyons pouvoir affirmer quil a existé des baculites-vertébrés de plus de quatre pieds de long, car ce qu'on en retire aujourd'hui du sein de la montagne ne présente que des fragmens plus ou moins longs, plus ou moins gros, et dont nous en avons vu qui avoient plus d'un pouce de diamitre à la base, tandis que des pointesou sommets que nous avons trouvés nous-mêmes, se terminoient en pointes d'aiguille; et comme la dégradation conique de cette coquille est pour ainsi dire insensible, nous croyons plutôt être resté en-dessous de la longucur, que de l'avoir excédéc.

Les cloisons lobées, festonnées, découpées du baculite vertébré, rappellent celles si sinueuses et si persillées de quelques cornes d'Ammon; lobement et persillures, qui ayant suffi aux conchyliologues pour former plusieurs genres parmi ces coquilles spirées, nous autorisent a former le genre suivant. 
Coquille droite, cônique, fistulée. 
546 COQUTLLES

LXXXVII'. GENRE.

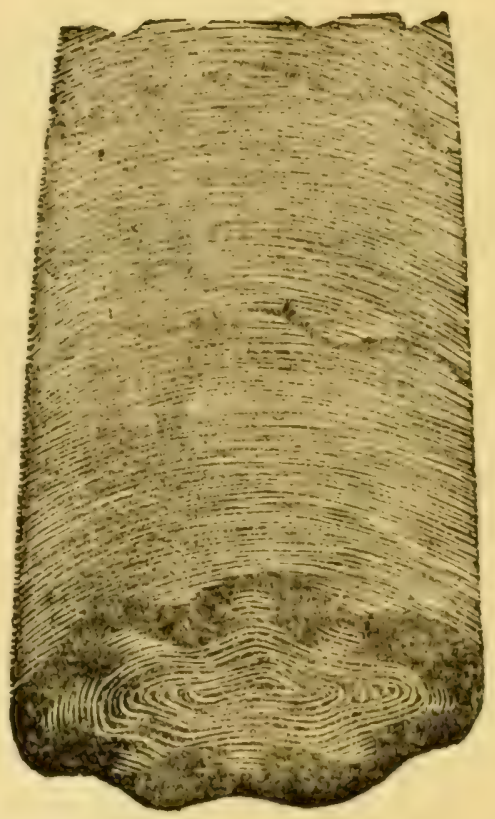

TIRANITE; en litin, Thantes. 


\section{LE TIRANITE.}

Caractères génériques. Coquille libre, univalve, cloisonnée; droite et en cône fistuleux; cloisons ondulées sur les bords; bouche ovale, ondulée, horizontale; sommet pointu; siphon central.

\section{Espèce servant de type au genre.}

\section{Le Tiranite géant. Tiranites gigas.}

Knorr, Suppl. pl. XII. fig. I à 5 .

Cette pétrification, déjà assez rare par elle-même, ne nous a jamais encore présenté son analogue marin ; il doit par conséquent être rangé parmi ces coquilles pélagiennes, qui vivent au sein des mers prolondes, et que des causes quelconques empêchent de remonter à la surface des flots. Les tiranites comme les baculites, ne se retirent du sein de la terre que par fragmens, mais il n'est pas impossible d'en réunir plusieurs, en raison de la diminution insensible et graduelle qui règne depuis la base jusqu'au sommet, que nous avons reconnu être pointu; et comme il y a des fragmens qui nous présentent jusqu'à trois pouces de base, et que leur dégradation n'éprouve point trois lignes sur un pied de longueur, quatre pieds ne peuvent avoir perdu qu'un pouce; ce qui nous donneroit douze pieds de long pour arriver d'une base de trois pouces à un sommet pointu 
ed ciliké. Ces calculs quoique étommins, n'en sont pas moins exacts; ils prouvent jusqu'd l'évidence à quelles grandes dimensions peuvent parrenir de certaines coquilles, et combien se prolonge la vie des mollusques qui les construisent : et ces mollusques ne doivent-ils point aussi parvenir à une taille monstrueuse, puisque tout nous prouve que semblables en ccla aux crocodiles, aux refuins, aux balcines, aux boas, ils croissent tout le temps de leur longue existence, et que chaque jour ajoute ì leurs dimensions colossales.

Knorr a gravé le tiranite géant avec le plus grand soin; la planche qui le représente est une des meilleures de tou son ouvrage, et il a consacré aux développemens de ce fossile une planche tout entière. Celui quil représcute est de couleur ocracée, mais cependant en majeure partie calcaire; de notre côté, nous avons rencontré des liranites dans la montagne de Ste. Catherine, pris de Rouen ; ils sont calcaires et d'une couleur jaunissante ; humectés par lhaleine, ils émettent une odeur argileuse, ainsi que cclui de Knorr ; ceux que nous trouvîmes sont ovales et naturellement aplatis.

En général nous ne donnons que des fragmens des coquilles droites, parce que beaucoup d'entre clles sont tris-allongies; cependant nous nous flattons que ces mímes fragmens, de la maniére que nous les avons dessinés, suflisent pour dommer au moins une idéc de la coquille que uous voulons déterminer. 
Coquille droite, intersectée. 


\section{LXXXVIII'. GENRE。}

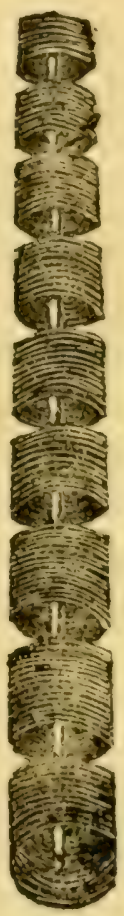

MOLOSSE; en latin, Morosst's. 


\section{LE IMOLOSSE.}

Caractères génériques. Coquille libre, univalve, cloisonnée; droite, conique, fistuleuse et intersectće; cloisons unies, faites en tambour ; siphon latéral, continu, rond, servant de bouche ; sommet pointu ; base horizontale.

Espèce servant de type au genre.

\section{Le Molosse parfilé. Molossus gracilis.}

Orthoceratites gracilis. Blumenbach, specimen archæologiæ telluris, terrarumque in primis hannoveranarum, pag. 21. tab. 2. fig. 6 .

Nous puisons cette coquille parmi celles que publia Blumenbach dans son essai pour servir à l'histoire du globe, qu'il fit imprimer in-4․ à Gœttingue, en 1 So5. Ce savant célèbre la regarda comme un orthocérate, mais d'après ses caractères individuels nous n'avons point pu lui conserver cette dénomination : et en effet les orthocérates de la Peyrouse, nommés actuellement hippurites et batolites, ne sont pas intersectés, mais ils offrent au contraire un iêt continu et solide; et l'orthocère de Lamarck est une coquille de la Méditerranée que ce savant prit dans Gualtieri, planche 19 , figures L. M. ( I). Il fut suivi par Félix de Roissy (2), qui réunit sous

(1) Lamarck, Syst. des anim. sans vert. pag. ro3. genre orthocère.

(2) Félix de Roissy, Hist. des moll., faisant suite aux oenres de Buffun, édit. de Sonnini, pag. 36 et suiv, tonı. V. pl. 5 I. fig. 6. 
le mème genre jusyüa neufespèces, matis celle ynil nous donne pour type est arquée, et les cloisons étant adhérentes les unes aux autres ne permettent plus a notre molosse de se joindre à clles: peut-ctre serons-nous forcés un jour de restituer le genre orthocère, tel que Lamarck et Frílix de Roissy l'ont désigné; mais nous croyons cependant que les coquilles indiquées sous ce ginre par ces conchyliologues, sont maintenant jetées par nous dans les genres glandiole, amimone et réophage qui prícedent; et comme cette lacune, si clle existe, ne sera point la seule (qui restera a remplir aprés nous, nous abanklonnons ce point de critique à tous ceux qui croiront devoir le relever; craignant dejà, et peut-itre avec raison, quion ne nous reproche d'aroir trop multiplié des gemres, qui, quoique nombreux, ne complètent point, à coup sûr, tout l'ordre conchyliologique.

L'aspect singulier du molosse parfilé avoit frappé Blumenbach au point qu'il avoue qu'il lui paroit énigmatique; il en possédoit un fragment d'un pouce et demi de long, qu'il regardoit comme parfaitement cylindrique, et qui avoit la grosseur d'une plume d'oie; converti en pyrite, cet échantillon provenoit des schistes argileux de la fosse, aujourd'hui abandonnée, et connue sous le nom du roi David du mont Traenkenberg, prés de Clausthal dans le Hartz. Les concamérations faites en tambour, sont conrexes en-dessus, et concaves en-dessous; et nous avons posiédé quelques fragmens qui avoient un demi-pouce de diametre. 
Coquille droite, cônique, fistulée. 


\section{LXXXIX: GENRE.}

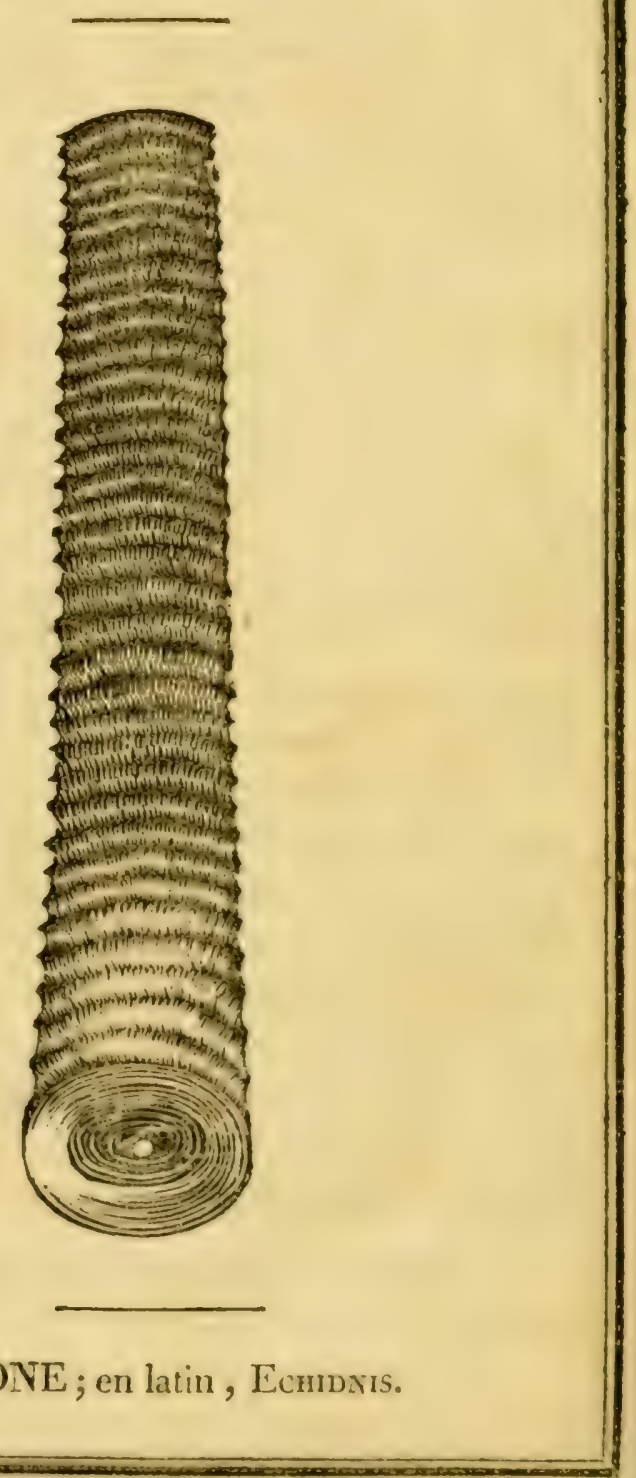




\section{L'ÉCHIDNE.}

Caractères génériques. Coquille libre, univalve, cloisonnée ; droite, conique, fistuleuse; bouche arrondie, horizontale ; sommet aigu ; cloisons plissées sur les bords seulement; siphon continu et central.

\section{Espèce servant de type au genre.}

\section{L'Échidne diluvien. Echidriis diluvianus.}

A l'époque où nous remplìmes les fonctions d'aidegéologue au Musée du Jardin des Plantes de Paris, sous les ordres de M. Faujas-de-St.-Fond, nous pûmes examiner de près les nombreuses pétrifications que renfermoient le magasin et les galeries de ce magnifique musée. Parmi ces pétrifications il existoit un bloc de marbre de trois pieds de long, poli sur une de ses faces et brut sur toutes les autres; il étoit gris, spathique, ondulé, et venoit de la vallée d'Os dans les Pyrénées. Ce marbre étoit uniquement et entièrement lardé du corps pétrifié, auquel actuellement nous donnons le nom d'échidne diluvien; à la vérité ce n'étoient plus que des fragmens, parce que ce bloc avoit été arraché du rocher, mais parmi ces fragmens il y en avoit de plus d'un pied de long, et ils étoient continus et sans fracture. A cette époque nous en fimes le dessin.

Ce dessin a passé avec beaucoup d'autres dans les portefeuilles si volumineux de M. Faujas-de-St.-Fond, qui, peu de temps après, reçut d'Angleterre un autre fragment d'échidne, d'une assez bonne longueur, de 
conleur brune et ocracée, parce quil provenoit diune exploitation de roche ocracéc et ferrugineuse quion convertissoit en fer.

Plus tard M. Dufiesne, revenant de Londres, y aroit observé dans la collection de IIunter, une coquille parasite des balcines, dont de Lamarck fit un genre sous la dénomination de tubicinelle ( 1 ). On crut un instant y pouvoir reconnoître l'analogne de l'échidne dilurien: mais cela ne peut être, la tubicinclle est une coquille dissivalve et uniloculaire; l'échidne est chambré, et il a un siphon: mais ce qui tranche toute difficulé c'est que les plus grandes tubicinelles connues nont que trois travers de doigt de longueur, et comme dans le bloc dont nous parlons nous arons pu étudier les échidnes, nous y en avons trouvé depuis la pointe d'une aiguille jusqu'au diamitre de seize lignes, ne perdant dans leur progression conique qu'une ligne par pied; ce qui nous donneroit plus de seize pieds de longueur. Dís-lors l'échidne n'est plus ce parasite implanté dans la superficic desbaleines, il les laboureroit au contraire d'une maniere effroyable; nous en ferons donc une coquille pédagienne, pritrifice, dont l'analogue marin n'est point encore commu; et dont nous arons domne ici un fragnent propre à constiluer les caractères du genre.

(I) Annales du Mus. d'hisionat. vol. $x$, pag. 46 r et 465 , et pl. 50. 
Coquille droite, cônique. 


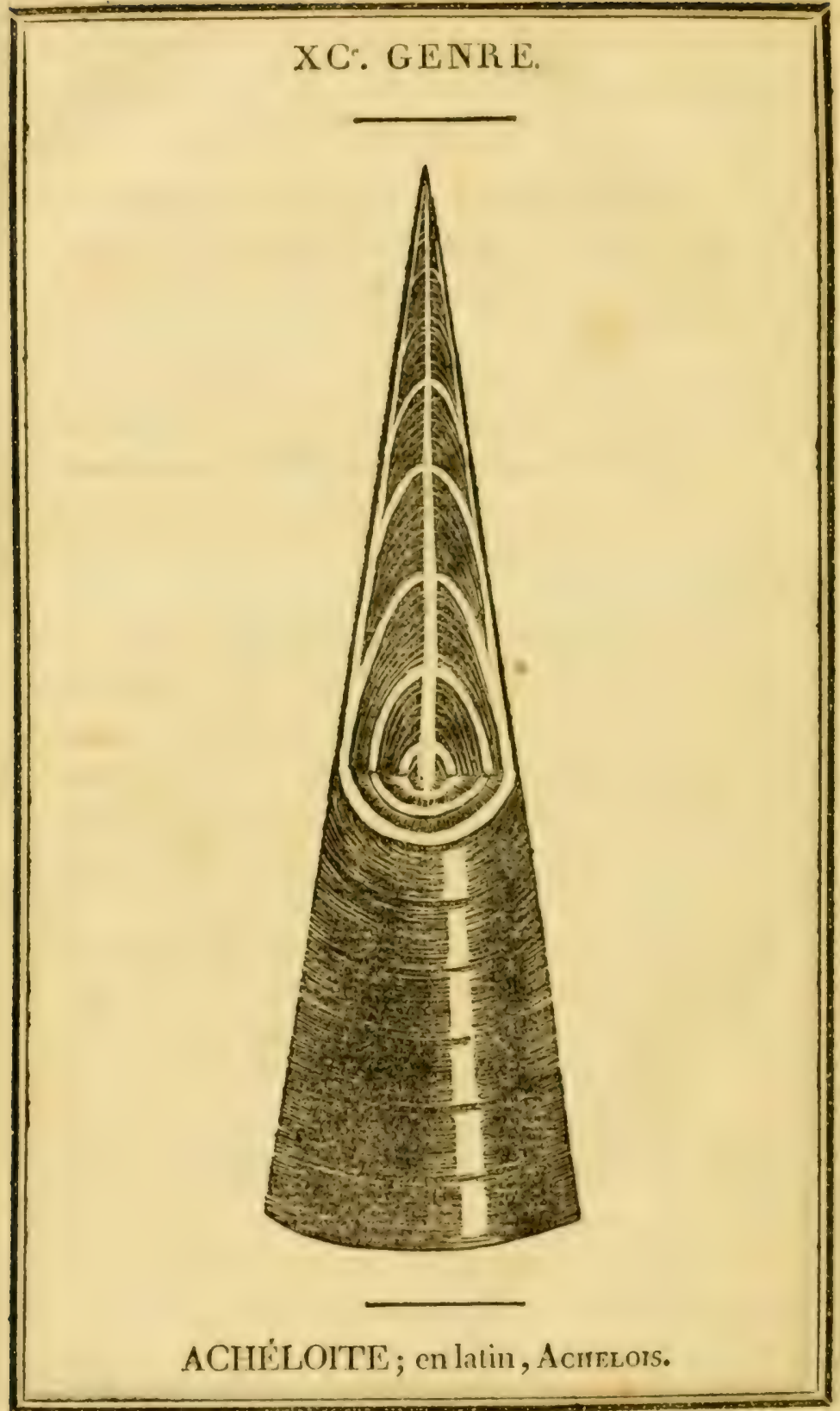




\section{L'ACHÉLOITE.}

Caractères génériques. Coquille libre, univalve, cloisonnée; droite et cônique; bouche arrondie et horizontale; siphon central, continu; cloisons côniçues, obtuses et unies.

\section{Espèce servant de type au genre.}

\section{L'Achéloüte pyramidal. Acheloïs pyramidans.}

Knorr, T.II. sect. I. pl. I I. A. VIII, et suppl. tab. IV'. fig. I.

Bien plus évasée à sa base que les bélemnites, cette coquille pélagienne fossile, et dont on ne connoît pas encore l'analogue, a à-peu-près les mêmes gisemens. Elle est seulement beaucoup plus rare, et forme un des beaux ornemens des cabinets d'oryctologie, attendu qu'ordinairement elle se trouve lardée dans des marbres solides et colorés, et qu'on se plait à en scier des tables où on la voit dans toutes ses coupes. L'achéloïte qui vient d'Altdorff est rouge, d'une teinte vineuse qu'il partage en partie avec le marbre brunâtre qui lui sert de gangue; son têt comme ses cloisons sont convertis en spath calcaire blanc qui tranche avec une netteté extrême, sur le fond des chambres ou alvéoles, ainsi que sur la pierre, bien plus fortement colorés; l'intérieur des concamérations est très-souvent rempli de cristaux spathiques qui tapissent lintérieur des cloisons, dont la partie du milieu est quelquefois môme dorée et changée en pyrites. 
Il existe daus les galeries du Jardin Impérial des Plintes de Paris, une plargue en carré longr de marbore à fond noir, qui rient de la vallíe d'Os aux Pyrénces : ce marbre est entiérement lardé d'achélöites, dont le tât converti en spath blanc, se dessine encore avec pureté sur un fond d'une couleur si opposcie; et cet échantillon est l'un des plus beaux qu'un professeur de géologic puisse présenter, alors quiil entretient ses auditeurs des marbres et des pierres, dans lesruels les types de l'organisation animale n'ont pas encore étć effacés par les âges, et par les rivolutions sans nombre que le globe rue nous habitons a ćprouvées i des époques plus ou moins ćloignces, ou plus ou moins rapprochées les unes des autres.

Nous comnoissons des achélö̈tes de pris de deux pieds de longueur; et jamais il n'est entré dans l'esprit de personne qu'ils aient constitué les pièces intéricures d'un bélemnite; observation que nous jetons ici par rapport au genre callirhoc', qui le suit immédiatement. 
Coquille droite, cônique. 


\section{GENRE.}

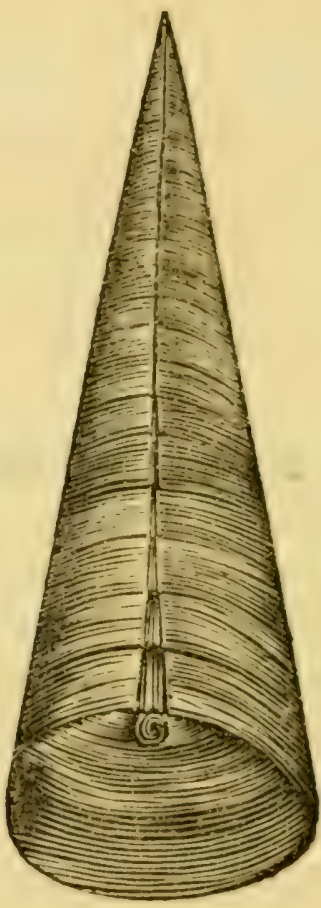

CallirhoÉ; en latin, Callinuoe. 


\section{LE CALLIRHOÉ.}

Caractères génériques. Coquille libre, univalve, cloisonnée ; droite et cônique ; bouche arrondie et horizontale, siphon latéral, continu; cloisons bombées et unies.

Espèce servant de type au genre.

\section{Le Callirhoé alvéolé. Callirhoe alveolatus.}

Orthocératite dont le siphon se trouve près de la circonférence..... Knorr, monumens, etc. T. II. Sect. II. pag. 242. pl. I*. figures 8 et 9 .

Cette pétrification, formée d'alvéoles empilées et liẻes entre elles par un siphon marginal et continu, est représentée ici de moyenne grandeur; plus petite, on la trouve quelquefois dans la bouche ou ouverture des bélemnites, et c'est ce qui a fait conjecturer à quelques auteurs que ces alvéoles faisoient partic des bélemnites. Nous ne partageons point cette opinion, d'autant plus qu'en premicr licu on ne trouve que très-rarement ces alvéoles dans les bélemnites, et comme rien n'est aussi commun qu'eux dans le règne des pétrifications, pourquoi ne les y retrouveroit-on point constamment si elles n'y étoient point parasites? D'un autre côté, ces alvéoles en général assez épaisses, ne coïncident point par la séparation de leurs cloisons avec celles des bélemnites qui les recèlent. D'ailleurs l'ensemble de ces alvéoles dont nous faisons le genre callirhoé, n'est ordinairement point de la mème substance que le bélemnite, car tandis 
que celui-ci sera spathinge et calcaire, le callinhoe se: trouvera pyriteux; preure dune autre organisation. Et enfin en dernière analyse, l'achíloite ne preisente-t-il pas la même construction que le callirhoé, a l'exception du sjplion qui est central dans le premier, tandis quil est marginal dans le second ; seule différence caracléristique daus ces deuxgenres? Nous ignorons si ce sont les mémes rues qui auront porté Walch à regarder comme nous le callirhoé alvéolé comme n'appartenant point ì des bélemnites; car aprés aroir rapporté en fireur de lopinion contraire, celle de M. d'Amnone, il dit expressément: "Que les numéros 8 et g de la planche citce, lui paroissent ère des fragmens d'orthocératites, dont le: siphon se trouve prés de la circonférence ..... quil a plusicurs orthocératites avec de prareils siphons [ul)erenleux dans son cabinet : et qu'on les trouve ruelyucfoi: dams le pays de Mleklembourgy ». Ceux de M. d Annone, colorés en rouge par le fer, venoient didistorf, dans le canton de Bâle.

Le callirhoe alvéolí que nous pulblions rient d'Alldorfl: ses cloisonssont pyrirseres, ses chambresou alréoles

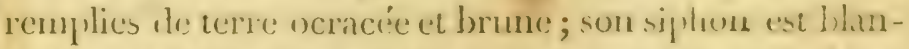
chattre. Lorsque les cloisons sont détachies et isoliees, elles constituent les verres de montre de quelques auteurs. 
Coquille droite, cônique, fistulée. 


\section{XCII'. GENRE.}

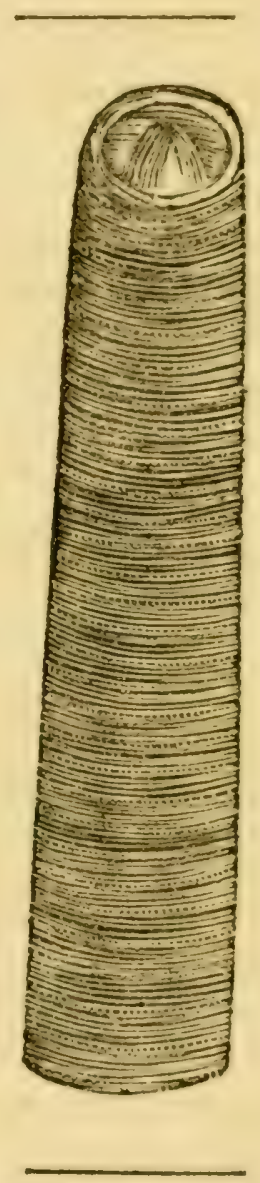

TÉLÉBOITE; en latin, T'reenors. 


\section{LE TÉLÉBOITE.}

Caractères génériques. Coquille libre, univalve, cloisonnée, criblée à chaque camération; droite et cônique ; bouche ronde, horizontale ; siphon central ; cloisons côniques et unies.

Espèce servant de type au genre.

\section{Le Téléboüte annelé. Teleboïs annulatus.}

Pierre colonnaire de Gothlande ..... Knorr, Monumens, etc. T.II. Sect. II. pag. I 22, I 25. planch. G. V. et G. VI. Colonnes articulées. Ibidem. - Klein, neue gesellschaffiliche Erzahlungen, tom. 4. pag. I 20.

Cette coquille, que l'on n'a encore rencontrée qu’à l'état de pétrification, a singulièrement embarrassé Klein et Walch ; on voit employer surtout au second de ces auteurs deux grandes pages in-folio dans la perplexité ou il se trouve, voulant absolument placer les téléboïtes parmi les entroques, et avoucr enfin en dernière analyse qu'il reste encore ici beaucoup à découvrir; mais si, avec sa sagacité ordinaire, Walch avoit seulement voulu considérer que ses pierres colonnaires affectoient une forme connique et terminée, que de plus elles étoient cloisonnées, il auroit pu dès-lors les ranger à côté des 
briemnites quedies viennent côloyer. Nous devous cependant recomnoître qüindépendamment de l'échantillon qui fait le sujet de celarticle, nous avous étéporté a constituer son genre par celui du porodrague, qui en est si voisin. Lille de Gotlande est pour ainsi dire entierement lardée de téléboiles, il s'y en rencontre de plus de trois pieds de long sur dix poures de dianietre, et comne ils y som spathiques, on en fait d'excellente chaux. Il y en a de blanes, de rouges, de cendrés; et on en rencontre en Angletere, qui sont pyriteux et noirs.

Liechantillon qui nous sert de type vient de la montagne de Ste.-Catherine, pries de Rouen; il est crayeuxcalcaire, ef de couleur jaunâtre, finement strié en rond, anmulé et criblé de petits pores, yui se rendent tous de la superficie au siphon central. Il a trois pouces et demi de long.

Nous devons remarquer, comme une chose trés-particulière, que les anneaux indicatifs des cloisons du téléboile annclé alterucnt ; tantôt ils sont pleins et tantôt criblís, dans un ordre trés-régulier, et serrément empilés les mons sur les autres. La tìte de notre échantillon est fruste, alin de laisser roir l'organisation intéricure. 
Coquille droite, cônique, sommet percé. 


\section{GENRE.}

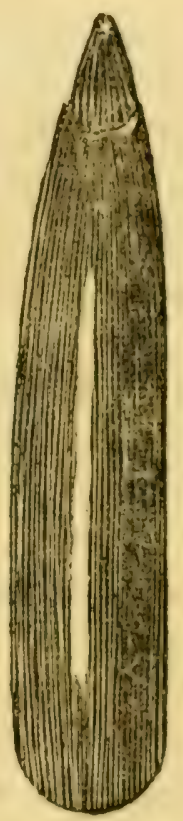

CÉTOCINE; enlatin, Cetocis. 


\section{LE CÉTOCINE.}

Caractères génériques. Coquille libre, univalve, cloisonnée ; drotte et cônique ; bouche ronde, horizontale; siphon central; sommet percé par un spincter étoilé; cloisons côniques et unies.

\section{Espèce servant de type au genre.}

\section{Le Cétocine uni. Cetocis glaber.}

Bélemnite qui, à son extrémité pointue ... a une ouverture en forme d'étoile.... Knorr, monumens, etc. T. II. Sect. II. pag. $24 \mathrm{r}$. pl. I^. fig. 4 .

L'oryctologue Walch avoit reconnu que ce corps pétrifié se rapprochoit beaucoup des bélemnites, mais qu'il offroit des particularités qui n'avoientpeut-être jamais encore été décrites : en cela il étoit d'accord avec M. d'Annone de Bâle , qui possédoit cet échantillon dans son cabinet, et qui écrivit: "Qu'en I751, il avoit déjà découvert quelques morceaux de ces bélemnites singuliers sur la montagne de Muttenz, et dans la suite aussi aux environs de Prattelen; mais qu'elles y étoient fort rares, et que lorsqu'il en auroit un plus 
grand nombre, il essayeroit de les anatoniser, pour ('n commitre de plus pris la strucure; et que peut-être clles serviroient a réprandre puek fues lumieres sur J'origine de la bílemmitc en général, qui nonobstant la quantité immense quion en troure, n'est pas encore sulfisamment commue $\%$.

Nous ferons encore ici une autre observation sur le texte de Walch, car il dit que : le tè étant cassé par son extrémité pointue, laisse ì découvert une pointe ímonssée et amondie.... et une ourerture en forme détoile ». Mais Walch comme d'Amone, n'avoit vu que des fiagrnens plus ou moins roulés de cette corpuille, et pour voir cette ouverture étoilće ils durent eu rafraichir le bout; ce qui du reste proure que toutes les cloisons ont en partage cette ouverture. Pour nous, nous ayons la cerlitude que le bout de cette bilemnite présente cette ¿toile, et si dans notre dessin nous en avons découvert le sommet, c'est pour montrer la disposition des cloisons.

Notre cétocine uni vient de Namur; il est noir, spathique, fétide, et de trois pouces de long.

Ceux de Muttenz et de Prattelen sont de coulcur cen drée, ocracée et vineuse. 
Coquille droite, cônique; sommet percé. 


\section{XCIVe. GENRE。}

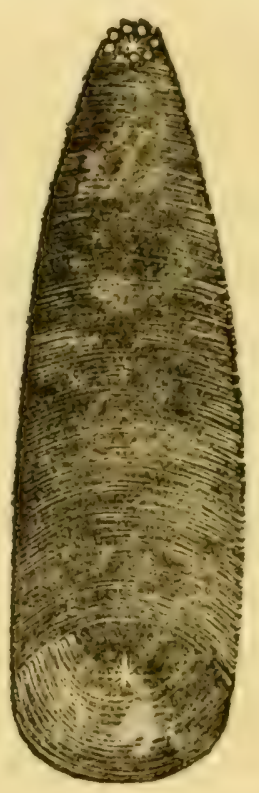

ACAME; en latin, AciMAs. 


\section{L'ACAME.}

Caractères génériques. Coquille libre, univalve, cloisonnée; droite et cônique ; bouche ronde, horizontale; siphon central; sommet percé de huit mamelons ou tubercules disposés autour d'un spincter étoilé; cloisons côniques, plissées dans leurs fonds; bords unis.

\section{Espèce servant de type au genre.}

L'Acame multiforé. Acamas polyforatus.

Knorr, monumens, etc. T. II. sect. II. pag. 2/1. pl. I^. fig. I , 2, 3 .

Ce fut encore Walch qui, d'accord avec d'Annone, nous fit le premier connoitre cette coquille singulière en la rapportant aux bélemnites. Nous ne répéterons point ici de nouveauce que nous avons dit en parlant du genre cétocine, concernant les frottemens qu'avoient éprouvés les échantillons cités par ces auteurs; mais trouvant chez l'acame des modifications nouvelles, nous avons dû naturellement y voir aussi une organisation animale particulière, et qui nécessitoit la formation d'un genre; parce que dès qu'un animal est autrement conformé qu'un autre, il ne peut plus lui être congénère. Telle est 
lat loi yui nons a successivement dirige dans la coupe al dans lirrection des genres que nons présentons à nos lecteurs. I ans chacun d'eux c'est l'organisation animale qui mous décide, parce qu'elle moule les formes tant extíleures quintéricures, et quoique tres-souvent, et notamment ici, nous n'ayons point vu, ni puroir, ces animaux, il n'en est pas moins rai que cers formes sont souvent assez caractériscées pour mette à labri de toute er'teur.

L'acame multiforépasse, comme les bélemnites, par des degrés d'accroissement successifs; spathirque comme cux, il en partageles couleurs.

D'Annone le trouva sur la montagne de Muttenz, el dans les environs de Prattelen, dans le canton de Bâle. 


\section{Coquille droite, cônique.}




\section{GENRE.}
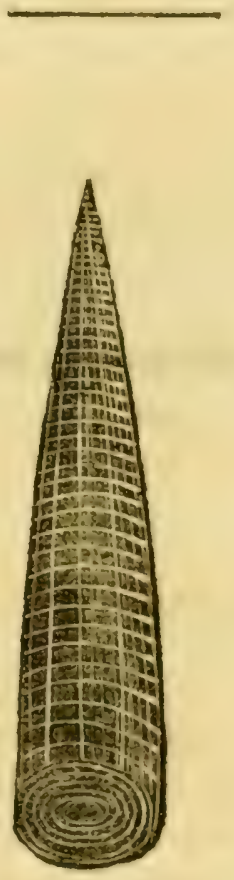

CHRISAORE; en latin, Crrrysaon. 


\section{LE CHRISAORE.}

Caractères génériques. Coquille libre, univalve, cloisonnée; cellulée dans toute sa longueur; droite; cônique ; bouche arrondie, horizontale ; siphon central, cloisons unies.

\section{Espèce servant de type au genre.}

Le Chrisaore hercinien. Chrysaor hercininus.

Vis à dix lames .... Knorr, monumens etc. tom. II. sect. II. pag. I33. pl. G. VII. fig. 4.

Entrainé par les formes extérieures et analogiques, Walch, rédacteur du texte qui accompagne les planches de Knorr, a regardé le petit échantillon du chrisaore d'Hüttenrode qu'il décrivit, comme pouvant appartenir aux genres des entroques et des astéries, ou lis de pierre, lilium lapideum. Il est vrai que cet échantillon étoit unique dans le cabinet du docteur Walch, et qu'on est souvent très-embarrassé lorsqu'on n'a point des objets répétés et de comparaison. Tout en se rendant infiniment utile à la science, cet auteur auroit dû avoir plus de confiance en ses forces, et voir que la forme cônique, se dégradant avec rapidité, et terminée en pointe, apparte- 
noit plus aux bélemmites qu'aux entrorliter. Il est rrai que la science bien plus avancie de nos jours, nous donne un arantage immense sur ces illustres devanciers, et que nous leur avons les plus grandes obligations sous le rapport de linitiative quils entreprirent si laboricusement. Quant it nous, nous rangeons re corps, jutlis marin et fossile, parni les corpuilles droites r't cluisonnées, parce rue nous avous pu en ćlul er un bel exemplaire de deux pouces et demi de long, et qui vient de la montagne de Ste.-Catherine, près de Rouen ; localité remarquable par les singuliers fussiles quielle renferme et qui sont bien loin encore d'être tous commus.

Le clirisaore hercinien de la montagne Ste.-Catlıcrine est calcaire et craycux, sa coulenr est d'un janne chamois, et ses cellules ouvertes à l'extérieur et dans toute sa longucur sont remplies d'une terre ocracćc.

Le chrisaore hercinien d'Tiittenrode est de conleur fortement ocracéc; sa substance spathique est totalement imprégnée de particules ferrugineuses, qui servent de gluten, et rendent ce spath presque aussi dur que le ' juartz; effet assez ordinaire aux molécules ocracécs. 
Coquille droite, cônique. 


\section{GENRE.}

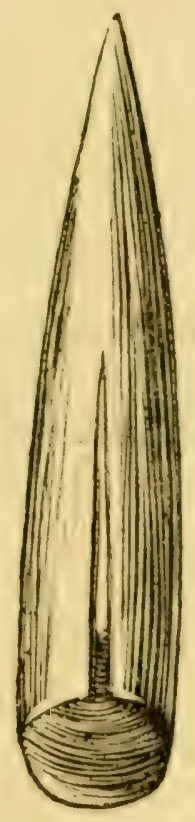

BELEMNITE; en latin, Ber.minites. 


\section{LE BELEMITE.}

Caractères génériques. Coquille libre, univalve, cloisonnée ; droite et cônique; bouche ronde, horizontale ; siphon central; cloisons côniques et unies; une gouttière sur le têt extérieur qui est lisse.

\section{Espèce servant de type au genre.}

\section{Le Bélemnite empilé. Belemnites paxillosus.}

Belemnites. Knorr, monumens, etc. T. II. sect. II. pag. 239 et suiv. planch. I. fig. I à 6 , et pl. I*. fig. 5, 6 . - Klein, de tabulis marinis, t. 8 , f. 2 à I 3. - Breyn, Dissert. de polyth. p. $4 \mathrm{I}$, t. I , no. I à I $4 .-$ Bertrand, Dict. des fossiles, art. belemnite. - David Erskin. Baker, phil. trans. art. 490. - Luid, Lithophyl. brit. p. 86. - Allion, oryctograph. pedemont. pag. 5o. Ehrhardt, Diss. belemnites suevic،, p. 36. - Kundmann, rariora naturæ et artis. - Brukmann, de belemn. musei sui. cent. I. epist. n. 65. - Richter, Museum. Frisch, museum hofmannianum. - Davila, catalogue raisonné. - Baier, oryct. norica, pag. 65. - Delamarck, Sist. des anim. sans vert. , pag. I 04 , genre bélemnite. Felix de Roissy, Hist. des Moll. , suite de Buffon, édit. de Sonnini, tom. V, pag. 43, genre bélemnite. $-\mathrm{Et}$ une foule d'auteurs dont la seule nomenclature occuperoit quelques pages.

En françois, Bélemnites, pierres de foudre, pierres fulminaires, pierres de tonnerre, pierre de lynx. Chez les anciens, lyncurium, lapis lyncis, dactylus 
illens, cerannias, cerannita, cunens toniumi, litpis finminans, oxyrinchus, corybantes, sagiua, jaculum, telum. - En grees, belos - En allemand, belemuit, lurhscteine, storchsteine, rappensteine, pícilsteinc, donnersteine, strablsteine, dommerstrahl, tenfelstegel, alpschosse. alpstcine, fingerstrine, pleillormige mcercelorensteine, zaplensteine, lucksteine, twarstrine. - En hollandois, belemmiet, pylsteen, straalsteen, donderstern, ravensteen, losteen. - En bel. se, donderkegel, vinger, duireh vinger. - Fin danois, veltelindserme, tordenseen, losteen. - Ell suédois, belemmiter , lossten, korpsten, tapyusen, thorvigrer, disten, alpsten, pilsten. - En anglois, belemnites, belenites, thunderbotes, thmmlerstones, arrow stoncs. - En écossois, ell-arrow, cll-arrow-heads. En italien, belcmmite, balenite, betulo, ceramic, pietre del tuono, pietre del fulinine, saclte. - En espagnol, belemuitas, pietra del rayo o centella, datil. Ein portugais, belemuilas, pedras de trovao, pedras de lynce. - En polonois, komien palczasty. - En boluémien, hromowy kamen.

On rencontre des belemmites repuis la grosseur d'un grain d'aroine jusıfüa celle du bras, et de plus de deux pieds de longueur. Elles sont des coyuilles pélaziennes, extrêmement répandues dans toutes les parties calcaires of formeginemsa's du globe : on les y trouve dans lous les chats fossiles de pétrifications; calcares, agatisées, firruginnuses, pyritisées sous tous les modes et sous toutes les modifications quil seroit possilsle de simaginer. Lanalogue vivant est encore inconmu. 
Coquille droite, lancéolée. 


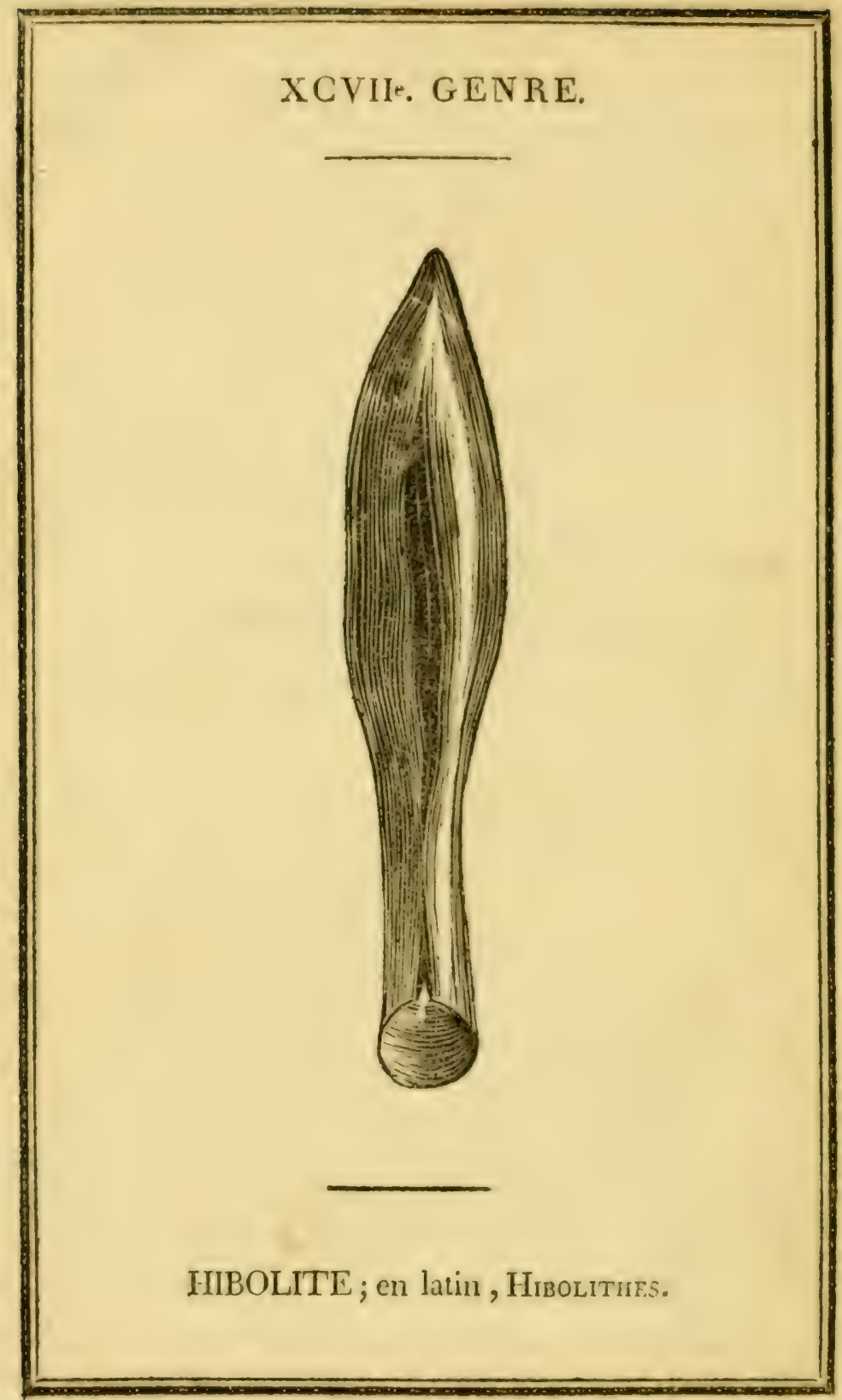




\section{L'HIBOLITE.}

Caractères génériques. Coquille libre, univalve, cloisonnée; droite, renflée en fer de lance; bouche ronde, horizontale; siphon central ; cloisons côni fques, unies, d'abord aplaties, ensuite rondes; une gouttiere sur le têt extérieur qui est lissé.

\section{Espèce servant de type au genre.}

\section{Hibolite lance. Hibolithes hastatus.}

Bélemnite en forme de lance. Felix de Roissy, hist. nat. des mollusq., faisant suite aux œurres de Buffon, édit. de Sonnini, tom. V, pag. 48.

D'après sa configuration en fer de lance, et en ayani reconinu trois espèces particulières, nous nous sommes décidés à turer l'hibolite des bélemnites avec lesquels il a été confondu jusquàa ce jour. Tout en lui nous prouve une organisation qui ne fut pas commune à son mollusque avec celui des bélemnites; non-sculement renflé en partant du sommet, il s'étrangle ensuite pour former une bouche ronde; mais en général son mode de pétrification n'est plus le même que celui des bélemnites ; il n'est plus corné, il tient davantage du marbre, et sa couleur est noire. Il répand aussi moins de fétidité lor'squ'on le frotte.

Nous avouons que l'histoire naturelle des bélemnites 
demande impérieusement d'ètre débrouillée; et sur cet objet on pourroit consulter des écrivains du plus grand mérite (1): mais ici, trop resserrés, nous n'avons point pu nous en occuper comme nous l'eussions voulu, et nous avons pris le parti de renvoyer cette partie pour le moment ou nous pourrons, après a voir établi les genres, nons occuper des espéces. Parmi les auteurs que nous citons, les uns ont éclairci la matiere, les autres l'ont embrouillée; et en le faisant ils nous ont préparé de nouveaux travaux.

L'hibolite lance vient principalement des environs de Gap ; nous en avons possédé de trois pouces et demi de longueur; constamment nous l'avons vu en consistance de marbre, plutôt qu'en celle purement spathique, et de couleur noire. Il est poli à sa surface et cependant un peu fendillé.

(r) Breyn, de polythalamiis.

Kleyn, descrip. tubul. marinorum.

Knorr, monumens, etc.

Sage, journal de phys. brumaire, an 9 .

Felix de Roissy, loco cit. pag. 48. 
Coquille droite, lancéolée. 


\section{GENRE.}

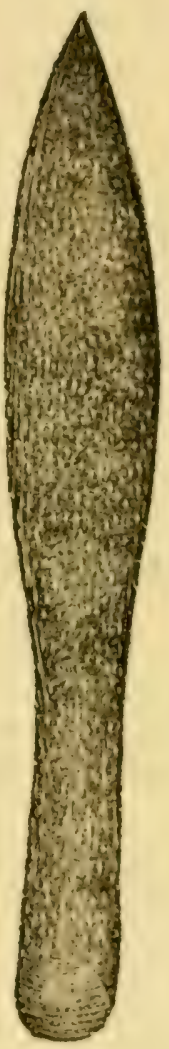

PORODRAGUE; ch latin, Poromacis. 


\section{LE PORODRAGUE.}

Caractères génériques. Coquille libre, univalve, cloisonnée; droite, renflée en fer de lance arrondi; bouche ronde, horizontale; siphon central ; cloisons côniques, unies; une gouttière sur le têt extérieur, qui est criblé de pores allongés.

\section{Espèce servant de type au g'enre.}

\section{Le Porodrague restitué. Porodragus restitutus.}

Félix de Roissy, hist. nat. des moll., faisant suite aux œuvres de Buffon, édit. de Sonnini, vol. V , pag. 48 .

Depuis long-temps IM. Faujas-de-St.-Fond possédoit dans son riche et nombreux cabinet deux ou trois petites coquilles fossiles, assez rapprochées des bélemnites pour les confondre avec eux, frustes et entièrement criblées à l'extérieur de petites taches blanches, allongées, que nous regardâmes il y a sept à huit ans, comme autant d'anciennes loges formées par une très-petite e spèce de pholade. Ce savant respectable les avoit recueillies dans les environs de Gap, età cette époque il voulut bien les soumettre à notre examen; mais il ne put être que superficiel, d'autant plus que nous ne pûmes ni briser, ni mutiler ces échantillons, et qu'en conséquence il nous fut impossible d'en étudier à fond l'anatomie. Mais maintenant possédant de ces objets nous-mêmes, en ayant même de plus grands que ceux que nous venons de citer, nous en avons, pour l'avantage de la science, 
disposé comme de notre bicn, el en conséquence ayant recommu :

10. Que ces pores étoient réguliers et disposcis ave régularité; ce qui ne seroit pas, sils étoient l'ouvrage de pholades, qui perçant ca et li auroient formé des trous plus ou moins grands suivant leur ìge;

20. Quils sont aussi larges à l'extérieur quà une certaine profondeur; ce qui ne seroit pas siles pholactes les avoit creusés, le pliolarle croissant à mesure quil avance et élargissant son conduit;

5o. Que cespores ne pénétrent pas an centre; ce qui auroit eu licu danstous lessens si ces pholades les aroient percés;

Nous avons cru pouroir en conclure quils étoient l'ouvrage du mollusque créateur de la coquille, et en faire un genre particulier.

Le porodrague restitué est tantòt noir, tantòt gris ; nous en possédons de trois pouces de long sur huit lignes dans le plus fort diametre, à pores bien ouverts, de substance calcaire spathique, et respirant fortement l'argile.

C'est un fossile sans analogue, 
Coquille droite, cônique. 


\section{XCIXe. GENRE.}
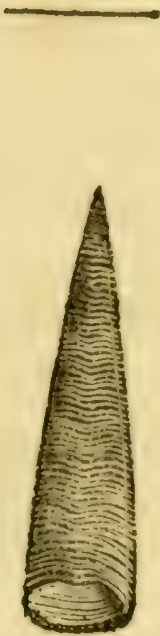

PIRGOPOLE; en Iatin, Prncorolox. 


\section{LE PIRGOPOLE.}

Caractères génériques. Coquille libre, univalve, emboittée ou cloisonnée; droite; sommet aigu; bouche arrondie, horizontale , aplatie; cloisons côniques, unies; déprimée sur ses deux flancs; siphon inconnu ou nul.

Espèce servant de type au genre.

Pirgopole de la Meuse. Pyrgopolon Mosæe.

Parmi les nombreuses pétrifications qui se trouvent dans l'intérieur de la montagne de Maëstricht, on en rencontre une très-singulière, encore à l'état crayeux ou de carbonate calcaire, point pétrifiée absolument, mais seulement fossile, blanche, et partageant cet état avec les nombreux mordans ou pattes de crabe qui y sont en si grande quantité. Ce fossile, quoique extrêmement nombreux dans ces endroits, a embarrassé tous les oryctologues, et en général ils ont préféré de n'en point parler. Il y, est plus nombreux encore que les mordans de crabe, et rassemblé par milliers dans le même entassement sans aucun de ces mordans; couché dans tous les sens, il n'est entremêlé d'aucun autre débris qui pourroit faire soupconner qu'il auroit fait partie de quelque crustacé. Après un mur examen, nous nous sommes décidés à le restituer aux mollusques testacés et chambrés auquel il appartient. 
Le piropole de la Meuse est formé de plusieurs tèts, ce quion recommoit facilement, lorsque les sommets fracturés permettent de lire le nombre de ces têts disposés en recouvrement; a l'extéricur il est strié dans le sens horizontal, et ces stries indirquent l'accroissement de la coquille qui est déprimée des deux còtés, ce qui dome la mème figure a la bouche. Cette forme est constante, et rappelle celle d'un bélemnite aplati.

Il est presque toujours entier, d'une belle conservation, blanc, un pou ocracé, ct il est rare d'en rencontrer de plus grands que de quinze lignes, beaucoup sont au-dessous de cette longueur. Il paroit d'apries leur gisement, que les pirgopoles vivoient en familles comme les bélemnites, les hippurites, etc. , à une époque éloiguée dans les àges. Nous n’en connoissons point l'analogue marin, à moins qu'on ne voulùt le retrouver dans les tuyaux testacés de quelques annelides. 
Coquille droite, torse. 


\section{Ce. GENRE.}

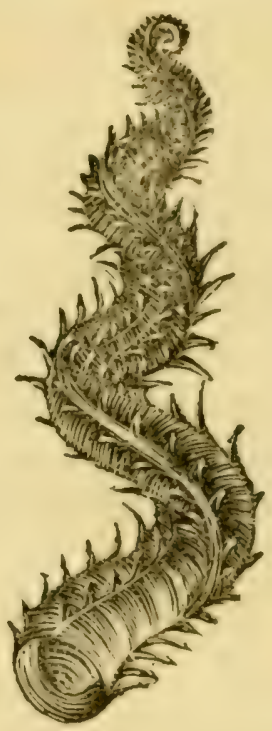

AGATIRSE; enlatin, Agatmrises.

DENYS DE MONTFORT, ommes delineavit, pinxit el sculpsit. 


\section{L'AG A TIRSE.}

Caractères génériques. Coquille libre, univalve, cloisonnée ; droite, mais torse, en forme de spire libre; fendue dans toute sa longueur; sommet aigu; bouche ronde, horizontale; cloisons unies; siphon nul.

\section{Espèce servant de type au genre.}

Agatirse furcelle. Agathirses furcellus.

Solen anguinus. Cappaad of bia ulor. Rumph, Amb. pag. I 25 de l'édit. holl. et pl. XLI, lettre E. - Dela Marck, syst. des anim. sans vert. pag. I 4 . observ. furcelle. - Serpula polythalamia. Nonnul. - Siliquaria anguina. Siliquaire de Grignon. Faujas de St.Fond, essai de géologie, tom. I, pag. 87 et suivantes, planche III, fig. 6,7 .

On trouve cet agatirse fossile à Grignon, près Versailles; il y està l'état crétacé, et de la plus belle conservation, quoique rare. La même rareté existe pour lui encore aujourd'hui dans l'état vivant, car on le retrouve parfaitement analogue sur les rivages de l'ile d'Amboine, ou Rumphius, magistrat vénéré et naturaliste respectable, le reconnut, comme on peut s'en assurer en comparant la figure qu'il en donna, celles publiées par Faujas de Saint-Fond, et celle que nous arons gravée. 
Le genre agatirse vient teminer pour nous la longue série des coquilles cloisomées ; ordre dejà existant, ou indiqué à la vérité, mais qui s'est consolidé sous notre plume. A peine y comnoissoit-on, à peine y rapportoiton une dixaine de genres; et cependant en portant ces genres à cent nous avons somvent fait des sacrifices. C'est un hommage que nous rendous au temps à venir ; et dans d'autres mains ces genres se multiplieront, comme ils auroient pu le faire dans les nôtres.

Encore peu cultivée, la carrière que nons venons de parcourir, est peut-ćtre la plus laboricuse de toutes celles qui nous restent à remplir; et c'est dis notre début que nous avons voulu netloyer celle route de toutes les pierres d'achoppement dont elle étoit semée; nos lecteurs, cenx pour qui nous arons écrit, jugeront si nous avons réussi.

Maintenant nous allons entrer dans un ordre de coquilles plus commues; ce sont celles qui ne sont point chambrées ni cloisonnćes; et nous arrivons à leurs genres plus nombreux encore par l'agatirse furcelle, qui dans une dégradation que nous arons essayé de rendre insensible, est devenu pour nous un chainon central, mais intermédiaire.

FIN DES COQUILLES CLOISONNÉES. 


\section{TABLE}

POLYGLOTTE ET SYNONYMIQUE

DES GENRES ET DES ESPEECE DE COQUILLES CONTENUS DANS CE VOLUME.

A.

Aur's Stock, 279 .

Acamas, 374 .

Acamas polyforatus, 375 .

ACAME, 374 .

Acheloïs, 358.

Acheloïs pyramidans, 359 .

ACHÉLOITE, 358.

Eolides, 142.

Eolides squanmatus, 143.

AGANIDE, 3 o.

Aganides, 30 .

Agathirses, 398 .

AGATIRSE, 398 .

Alpschœssc, 384.

Alpsteine, 384.

Alpsten, 384 .

Alvéoles, 363.

AMALTE, 90 .

Amalthens, 9o.

Amalthens margaritatns, 91 .

AMIMOME , 326 .
Amimonus, 326.

Amimonus elephantinus, $32 \%$.

AMMONIE, 74 .

Ammonshorner, 76 .

Ammonshorn, 76 .

Ammonit, 76 .

Ammonita, 76 .

Ammoniter, 76 .

Ammonites, 74,76 .

Ammonites virgatus, 75 .

Ammonitotypolithe, 96 .

Ammonins lapis, 76 .

ANDROMEDE, 38.

Andromedes, 38.

ANGULITHE, 6.

Angulithes, 6 .

Antenor, 70 .

Antenor diaphaneus, $\boldsymbol{f}$.

ANTENORE, $7 \circ$.

Archaias, I 90.

Archaias spirans, 19 $\mathrm{r}$.

ARCHIDIE, 190. 
Arethusa, 302.

Arethusa corymbosa, 303.

ARÉTHUSE, 302.

Arrowstones, 384 .

ASTACOLE, 262.

Astacolithe, $9^{2}$.

Astacolus, 262 .

Astacolus crepidulatus, 263.

B.

BACULITE, 342.

Baculites, 342 .

Baculites rertebralis, 343 .

Balenite, 384.

BATOLITE, 334 .

Batolites, 334.

Batolites organisans, 334 .

Belemniet, 384 .

Belemnit, 334.

Belemnitas, 384 .

BELEMNITE, 382 .

Belemniter, 384.

Belemuites, 382 .

Beleunite fer de lance, 387 .

Belemnites paxillosns, 382 .

Belcmnires polimitus, 323 .

Belemnite recourbé, 3 ig).

Belenites, $38 \%$.

Belemitas, 334.

BELLEROPHE, 50.

Belderoplan, 5 o.

Belos, 38if.

Belscluccken, 76 .

Belslak, 76 .

Bergsclunecken, 76 .

Betulo, 384.
Bia cojin, 3 .

Bia papeda , 3 .

Bia ulor, 3̈g9.

Biscliofstebe, 2.79.

Bisschop staef, 279.

BISIPHITE , 54 .

Bisiphytes, 54.

BORELIE, I 70 .

Borelis, 170.

Borelis melonoìdes, 1716

CALliRHOE, 362 .

Callirhoe alveolatus, 363.

Camérine, i 55.

Cancride, 266.

Cancris, 266.

Cancris auriculatus, 267 .

CANOPE, 290.

Canopus, 2 go.

Canopus fabcolatus, 29 I.

CANTHARE, 298.

Cantharus, 298.

Cantharus calceulatus, 299.

CANTHLOPE, 46 .

Canthropes, 46 .

Cippaad, 399.

Canda cancri, 92, 237.

CELIBE, 306.

Cellanthus, 206.

Cellanthus craticulatus, 207.

CELLULIE , 206.

Cenchrites, 17 ?.

Ceratoildes, 76 .

Ceratoïdes articulatus, 279.

Ceraunias, 384. 
Céranie, 384.

Ceraunita, 384.

CETOCINE, 3 7o.

Cetocis, 370 ,

Cetocis glaber, 3 7r.

CHARI BDE, 106

Charybs, 106.

Charybs plicatus, $10 \%$.

Chelibs, 306.

Chelibs gradatus, 307 .

CHRISAORE, 378 .

Chrysaor, 378 .

Chrysaor hercininus, 379 .

CHRY SOLE,, 6 .

Chrysolitha, 76 .

C'hrysolns, 26 .

CIBICIDE, , 22.

Cibicides, 122.

Cibicides refulgens, 123.

CIDAROLLE, 110.

Cidarollus, I ro.

Cidarollus plicatus, III.'

CLAUSULIE, $7^{8}$.

Clausnlus, 178 .

Clausulus indicator, $\mathbf{7 9}$.

CLISIPHONTE， 226.

Clisiphontes , 226.

Clisiphontes calcar, 327 .

Cochlea margaritifera, 3 .

Colonnes articulécs, 367 .

Corne d'Ammon , 75,76 .

Corne d'Ammon carénée, gr.

Corne d'Ammon droite, 343.

Corne d'Ammon elliptique, ô7. ÉCIIIDNE, 354.

Corne d'Ammon turbinée, I r9. Echidnis, 354.

Corne de bélier, 76 .
Cornet chambré, 99.

Cornet de postillon, 99 .

Cornet de St.-Hubert, 99.

Corno d'Ammoni , 76 .

Corno de carneiro, 76 .

Corno de Hammon, 76 .

Cornu'Ammonis, 76 .

Cornu Ammonis spurium, 99.

Coruucopix, 287 .

CORTALE, 114 .

Cortalus, 1 I 4 .

Cortalus pagodus, x 15 .

Corybantes, 384.

Cuerno de Ammon, 76 .

Cuneus tonitrui, 384.

D.

Dactylus ideus, 383 .

Datil, 384.

DISCOLITE, 186.

Discolites, 186.

Discolithe, 155 .

Discolithes concentricus, $18 \%$

Donder kegel, 384 .

Donder steen, 384 .

Donnersteine, 384.

Donnerstrahl, 384 .

Drachensteine, 76 .

Duivels munt, 155 .

Duyrels vinger, 334 . 
Egeon, 166.

ÉGEONE, I6G.

Egeon perforatus,, 6 ;

Ell arrow, 384.

Ell-arrow-heads, 384 .

Elfsten, 38 4.

ELLIPSOLITE, 86.

Ellipsolithes, 86.

Ellipsolithes funatus, 87 .

ELPHII)E, 14.

Elphidinm, 14 .

ËULIDE , 142.

HPONIDE, 126.

Eponides, 126.

Eponides repandus, 127 .

F.

Falurkiittcl, 3.

Fahrküttelsteine, 4 .

Fiente de mouette, 76 .

Fingersteine, 384.

Flache Schiffer, 259 .

FLORIL.IF, 134 .

Vlorilus, 134 .

Florilus stellatus, 135 .

Forsteenede slanger , 76 .

Furcelle, 399.

$$
\text { G. }
$$

Geophonus, 18.

GLOPONE, 18.

Gerade Hoerner, 287 .

Geribbte Schiffer, 223.

GIROGONITE, 382 .

Girogronites, 182.
Girogoniles medicaminnla, 183.

GLANDIOLE, 314.

Glandiolus, $3: 4$.

Glandiolus gradatus, 3,5.

\section{II.}

Haımonita, 76 .

HELEN DE, 194.

Helenis 194 .

Helenis spitosus, $19^{5}$.

Hélicite, 155.

Hélicite rayonné, 163.

Helmintholithus, 207 .

Herion, 130.

HERIONE, 3 ก.

Herion rostratus, 23 r.

HIBOLITE, 386 .

Hibolithes, 386.

HIPPURITE , 286.

Hippurites, 286.

Hippurites bioculatus, 287 .

Homalocératite, 343.

HORTOLE, 282.

Hortolus , 282.

Hortolus convolvans, 283.

Hromony kamen, 38f.

Hueksteine, 384.

I.

ILOTE, ig8.

llotes, 198 .

Ilotes rotalitatus, 399 .

J.

Jaculum, 384 . 
JESITE, 102.

Jesites, I02.

Jesites vermicularis, , 3 .

K.

Kika lapia, 3.

Koerner nautilus, 203 .

Komien palczasty, 384 .

Korpsten, 384.

Krany modang, 3.

Kranse Schiffer, 203.

L.

Lagenula, 3 ro.

Lagenula flosculosa, 3 I r.

I.AGENULE, 3 ro.

LAMPADIE, 242.

Lampas, 242.

Lampas trithemus: 243 .

Lapis lyncis, 383.

Lapis frumentarius, 155.

Lapis fulminans, 384 .

LICOPHRE, , 58.

LINTHURIE, 254.

Linthuris, 254.

Linthuris cassidatus, 255.

Lituit, 279.

LITUITE , $27^{8}$.

Lituiten, 279

Lituites, 278.

Lituites lituus , 279 .

Losten, 384 .

Losteen, 384.

Luchsteine, 384 .

Lycophris lenticularis, 159 .
Lycophris, 158.

Lyucurium, 383.

II.

MA CRODITE, , 238.

Macrodites, 238.

Macrodites cucullatus , 239 .

Méconnites, 17 I.

Meewendrek, 76 .

MELONIE, 66.

Melonis, 66.

Melonis etruscus, 67 .

Melonenschiffer, 17 !

MILLIOLITE, I 74 .

Milliolites, 174 .

Milliolites sabulosus, $17^{5}$.

MISILE, 294.

Misilus , 294.

Misilus aquatifer, $29^{5}$.

MOLOSSE, 350.

Molossus, 350.

Molossus gracilis, 35 r.

Monnoie de St.-Pierre, 155.

Monnoie du diable, 855 .

N.

Nauchel, 3.

Nautaliti, 4.

Nautici, 4.

NAUTILE, 2.

Nautile à deux siphons, 55.

Nautile à spire, 75 .

Nautile déprimé, 5 r.

Nantile encapuchonné, 3 r.

Nautile flambé, 3. 
Nautile ombiliqué, 59 .

Nautiliten, 4.

Nautilite ondulé, 64 .

Nantilite persillé, 63 .

Nautilites, 4.

Nautilite triangulaire, 7 .

Nautilo, 3.

Nautilus, 2.

Nautilus acutauricularis, 95 .

Nautilus aduncus, 195 .

Nautilus ambiguus, 23.

Nautilus angulatus, $x$ r 1 .

Nantilus asterizans, 135 .

Nautilus auriculus, 267 .

Nautilus calcar, $3 \mathbf{j}$.

Nautilus carinatus, 2/43.

Nautilus cassis, 255.

Nautilus crassus, 3 .

Nautilus craticulatus, 207.

Nautilus crepidula, 27 .

Nantilus crepidulus, 263.

Nautilus crispus, 203.

Nautilus elongatus, 26/t.

Nantilus farctus, I39.

Nautilus incrassatus, $21 x$.

Nautilus lenticularis, 159 .

Nautilus lituitatus, 27 .

Nautilus macellus, 15 .

Nautilus major , 3.

Nautilus mamilla , 156 .

Nantilus maximus, 3.

Nantilus melo, $67,17 \mathrm{x}$.

Nantilus orbiculus, 199.

Nautilus planatus, 259 .

Yautilus pompilius, 3 .

Nautilus pompiloïdes, 67 .
Nautilus repandus, 127 .

Nautilus sacer, 96 .

Nautilus spengleri, $\mathbf{x} 47$.

Nautilus spirula , 99.

Nautilus striatus, 203.

Nautilus strigillatus, 39 .

Nautilus vortex, Ir.

NOGROBE, 274 .

Nogrobs, 274.

Nogrobs vermicularis, 275 .

Nonion, zio.

NONIONE, 210 ,

Nonion incrassatus, $21 x$.

Numismales, 155 .

NUMULIE, 154 .

Nouulites denarius, $\times 55$.

Numulithes, 154 .

Nummus diabolicus, 155 .

Nummus sancti Bonifacii, , 55 .

Nummus sancti Petri, 155.

\section{O.}

OCEANIE, 58.

Oceanus, 58.

OEufs de poissons, I $7 \mathbf{r}$.

Oolither, 17 r.

Ophyocida, 76 .

Oplıyoïdes, 76 .

Ophyomorphita, 76 .

ORÉADE, 9 .

Oreas, 94.

Oreas subulatus, 95 .

Urthoceratlies, 28\%.

Orthoceratites gracilis, 35 r.

Orthoceratithes, 387 .

Orthokeratiten, 287 . 
Ovéolite, 175 .

Oxyrinchus , 384 .

P.

PACLITE , 3 I 8 .

Paclites, 318.

Paclites biforatrs, 3 rg.

Paerlemoer nautilus, 3 .

Parlemoer hooren, 3.

Parlslak, 3.

Papedasschnecke, 3.

Papedo hoorn, 3.

PATROCLE , 218.

Pacrocles, 218.

Patrocles querelans, 219 .

Pedras de lynce, 384 .

Pedras de trovao, 384 .

Pelagus, 62 .

PÉLAGUSE, 62.

PÉLORE, 22.

Pelorus, 22.

PÉNEROPLE, 258.

Peneroplis, 258.

Peneroplis lanatus, 25y.

PÉRIPLE, $27^{\circ}$.

Periples, 270.

Periples elongatus, $27 \mathbf{x}$ ? $^{2}$

Perlenmuxterhorn, 3 .

Perlschnecke, 3.

Pfeilsteine, 384.

PHARAME, 34 .

Pharamum, 34 .

Phacites, 155 .

PHONEME, 1 o.

Phonemus, ro.
Pierre colomnaire, 367 .

Pierre de fondre, 383.

Pierres de frais, I7 I.

Pierre de froment, 155.

Pierre lenticulaire, 155.

Pierre de lynx, 383.

Pierre de tonnerre, 383.

Picrre fulminaire, 383.

pietre del fulmine , 384.

Pietra del rayo o centella, 384 .

Pietre del tuono, 384.

Pilsten, 384.

PIRGOPOLE, 394 .

PLANULITE , 78 .

Planulites, 78 .

Planulites nndulatus, 79 .

POLLONTE, 246.

Pollontes, 246.

Pollontes vesicularis, 347 .

POLIXENE, 138.

Polyxenes, 138 .

Polyxenes cribratus, 139 .

Posthooren, 9o.

Posthorn, 99.

Posthoorntje, 99.

PORODRA GUE, 3go.

Porodragus, 3 go.

Porphites, 155.

Pylsteen , 384.

Pyrgopolon, 394 .

$\mathrm{R}$.

Raarenstecn, 384.

Radius articnlatas, 287 .

Ramshoorn, 76 . 
Rams'liorn, 76 .

Ramshorn sailor, 99.

Raphanister, 338.
Scortimus navicularis, 251 .

Seilfischisteine, $7^{6}$.

Serpens lapideus, 76 .

Raphanister campanulatum, $33 \mathrm{~g}$. Serpenti impetriti, -6 .

RAPHANISTRE, 338.

Rappensteine, 38 . REOPIIGE, 330 .

lieophax, 330.

Reoplax scorpiurus, 33 I.

RHINORURE, 234.

Rhinocurus, 234 .

Rhinocurusarancosus, $23 j$.

ROBULE, 214 .

Robulus, 214 .

Robulus cultratus, 2 r5.

Roggensteine, 171 .

ROTALITE, 162.

Rotalites, 162 .

Rotalites radiatus, 163 .

S.

Saclte, $38 f_{f}$.

Sagitta, 384 .

Sailor, 3 .

Salagramman, y6.

Schiffchen, 3 .

Schifferchen, 3.

Schiffliittel, 3.

Schipper, 3.

Schippertje, 3.

Schlangen, 76 .

Sclneclsensteine, 76 .

Scliwefelkiese, 155 .

SGOR'TIME, 250.

Scortimus, 250 .
Serpent stone, 76 .

Sirpula polythalamia , 399 . SIDÉROLITE, 1 jo.

Siderolithes, 150.

Siderolithes calcitrapes, I 5 r.

Siderolithes calcitrapoides, 151 .

Siliquaire de Grignon, 399 .

Siliquaria anguina, 399 .

SIMPLEGADE , 82 .

Simplegades, 82.

Simplerades colubratus, 83.

Sint Pieters nunt, 155.

Skeppare, 3.

Skibssnckien, 3.

Slangensteen, 76 .

Slangersteen, 76 .

Snake stone, 76 .

Snckkestein, 76 .

Solen anguinus, 399 .

SPINCTERULE , 222.

Spincterules, 222.

Spincterules costatus, 223.

Spirula, 98.

Spirula fragilis, 99 .

SPIRULE, $9^{\text {S. }}$

Spitzolirschiffer, $9^{5}$.

Sporilus, 42.

Spornschifter, 35.

SPORULIE, 43.

Steenliorn, 76 .

Steinhorner, 76 .

Storclisteine, 384. 
STORILLE, 130 .

Storilus, 130 .

Storilus radiatus, $13 \mathrm{I}$.

Straalsteen, 384 .

Strahlsteine, 384 .

\section{T.}

Tappsten, 384.

Taymanu samal, 90 .

Telum, 384.

Tenfelskegel , 384 .

Teufelspfennige, 155 .

Telebois, 366 .

Telebois annulatus, 367 .

TÉEEBITE, 366.

Thalamule, 322 .

Thalamus, 322 .

Thalamus polimitus, 323 .

Themeon, 202.

THEMEONE, 202.

Themeon rigatus, 203 .

Thorvigg, 384 .

Thunderbotes, 384 .

Thunderstones, 384.

TINOPORE, 146.

Tinoporus, 146 .

Tinoporus baculatus, 147 . TIRANITE , 346 .
Tiranites, 346.

Tiranites gigas, 347 .

Tordensteen, 384 .

Tubicinelle, 356 .

Tubulns concameratus, 279, 287. TURRILITE, 118.

Turrilites costatus, 119 .

Turrilites, 118 .

Turrilite turbiné, I 19.

Twarfsteine, $38 \%$.

$$
\text { V. }
$$

Vasulithe déprimé, $5 \mathbf{r}$.

Veltelindserne, 384.

Vinger, 384.

$V$ is à dix lames, 379 .

Voilier, 3.

W.

Wirbelschiffer, II.

Widderhoerner, 76 .

Widderlrorn . 99.

\section{Z.}

Zaglick, 76 .

Zapfensteiue, 384:

Zicherhoerner, 76 . 


\section{ERR A T UM.}

Page 2, figure première, dans quelques exemplaires la figure est retournéc; ce nautile doit montrer sa bouche en-dessous. 


SMITHSONIAN INSTITUTION LIBAARIES 